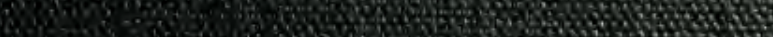

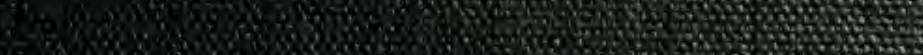

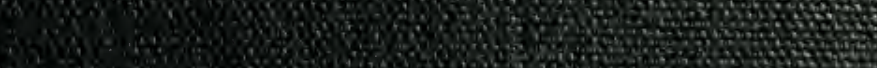

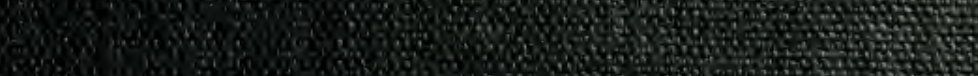
6.0. W.

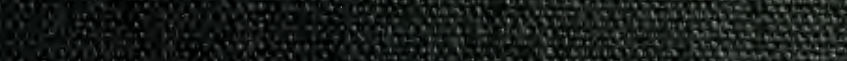

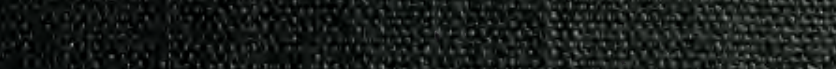
1.0.

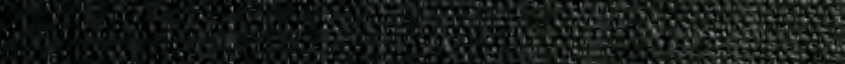
196. W.

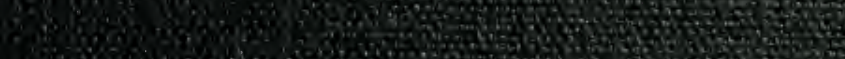

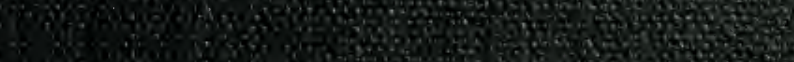
(40.5) 


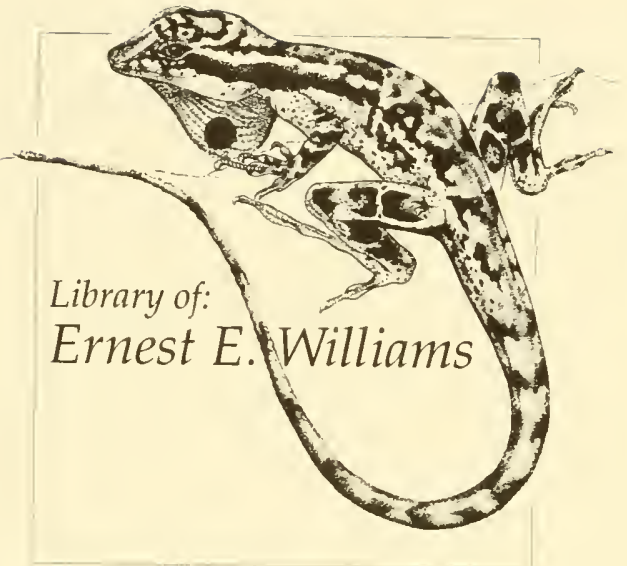




$$
\text { Emet at as and }
$$

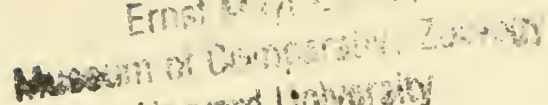
rowad lothes<smiles>C1=CC2CCC(C1)C2</smiles> 



\section{C. Z. - Herpetology}

$13,20 \%$

\section{KATALOG}

DER

\section{REPTILIEN - SAM M LUNG}

IM

\section{MUSEUM}

DER

\section{SENCKENBERGISCHEN NATURFORSCHENDEN \\ GESELLSCHAFT}

IN

FRANKFURT AM MAIN.

II. TEIL

(SCHLANGEN).

Von

Prof. Dr. 0. Boettger.

TAbgeschlossen Mitte Mai 1898.

Frankfurt a. M.

Druck rou Gebrïder Knauer. 



\section{KATALOG}

1) ER

\section{RE P'TILIE N - SAM M LUNG \\ IXI}

\section{MUSEUM}

DER

\section{SENCKENBERGISCHEN NATURFORSCHENDEN} GESELLSCHAFT

11

FRANKFURT AM MAIN.

II. TEIL

(SCHLANGEN).

Prof. Dr. O. Boettger.

Abgeschlossen Mitte Mai 1898.

Frankfurt a. $M$.

Druck von Gebrüder Knaner. 



\section{Einleitung',}

Mit dem vorliegenden Hefte ist die Reihe der herpetologischen Kataloge des Inseums der Senckenbergischen naturforschenden Gesellschaft abgeschlossen. Der "Katalog der Batrachier-Sammlnng" erschien Hitte August 1892, während der erste Teil des "Kataluges der Reptilien-Sammlung", der die Rhynchocephalen, Schildkröten, Krokodile, Eidechsen und Chamäleons entläilt, Mitte August 1893 veröffentlicht werden kounte.

In Dr. Eduard Rüppell's „Verzeichnis der in dem IInseum der Senckenbergischen naturforschenden Gesellschaft aufgestellten Sammlnugen. Dritte Abteilung: Amphibieu. Frankfurt a. M. Gedruckt bei Joh. Dav. Sanerlünder 1845, 4º 24 pagg.", das die Kriechtiere und Lurche umfaßt, sind die Schlangen auf Seite 14-21 aufgezählt. Danach waren vor 55 Jahren - im August 1843 - von Ophidiern im Museum zur Aufstellnng gekommen:

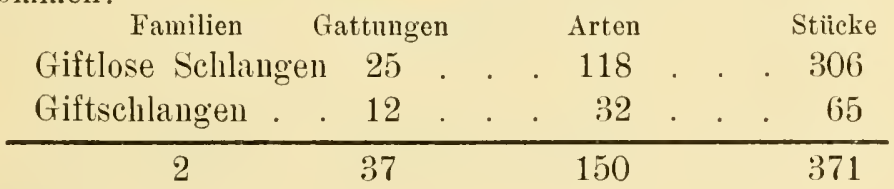

Alle diese Arten umd der größte Teil der alten Stïcke liegen hente noch mit ihren Originaletiketten in nnserer Sammlung. Über die Schenker dieses Grundstockes hat Herr Dr. Rüppell schon im Vorwort zu dem genannten ersten Verzeichnisse Mitteilmngen gemacht, die ich schon im I. Teile des "Katalogs der Reptilien-Sammlung" pag. IV erwähnt habe. Zu den dort erwähnten guitigen Gebern kommen ans älterer Zeit noch die längst verstorbenen Herren Herzog Max E. J. N. von Leuchtenberg in München und W. A. Bernus, W. Rosenbach, Dr. W. Schneider, Gebrüder G. \& M. Worms mit Frau vou Panhuijs, sämtlich aus Frankfurt a. M., hinzı. 
Zu der großen Anzahl von neneren Schenkern, deren Namen im "Katalog der Reptilien-Sammlung, I. Teil “ pag. IV-V und pag. 125-129 aufgezählt worden sind, treten noch folgende, deren Gaben durch Zahl, Seltenheit oder Schönheit besonders hervorragen:

Herr Max Bamberger, Kaufmanu in Frankfurt a. M.

" Dr. med O. Benecke in Deli, Sumatra.

" Dir. Dr. Cárlos Berg in Buenos-Aires, Argentina.

n J. Blum, Oberlehrer in Frankfurt a. II.

"Ferdinand Emmel, Kanfmann in Hamburg.

" Friedrich Emmel, Kanfmann in Arequipa, Peru.

Herren Gebr. Egbert \& The odor Engellar d in Deli, Sumatra.

Herr C. Eylandt, Naturalist in Askhabad, Transkaspien $\dagger$.

"Dr. Finger, Oberlehrer in Frankfurt a. M. †.

"Joll. von Fischer" in Montpellier, Hérault.

" Rud. Henrich, Braner in Frankfurt a. M.

"Gust. Herath aus Erankfurt a. M.

" Dr. L. ron Heyden, Major z. D. in Frankfurt a. M.

"Ferdinand Knobla uch, Konsul in Frankfurt a. M. †.

"Otto Koch, Kaufmann in Cebí, Philippinen $†$.

Frau H. Krämer, Wwe. in Frankfurt a M.

Herr F. C. Lehmann, Konsul in Popayán, U. S. Columbia.

”Dir. Dr. Heinrich Lenz in Liibeck.

" E. Lörscli in Frankfurt a. M.

"Dr. Löwentlial in Frankfurt a. MI. †.

Das Naturhistorische Museum in Madras, Brit.-Indien.

Herr F. Ma uß, Konsul in Valencia, Venezuela.

"Fr. de Mimont, Gutsbesitzer in Nenochóri, Euböa $\uparrow$.

" Dr. med. Pauli, Hofrat in Frankfurt a. M. †.

" O. Retowski, Staatsrat in Theodosia, Krim.

" Prof. Dr. Ferdinand Richters in Frankfurt a. M.

" Dr. med. H. Schädle in Casablanca, Marokko.

" Prof. Dr. Oskar Sclneider in Dresden.

"G. von Schröter, Konsul in San José, Costa Rica.

"Albrecht Seitz, Kanfmann in Hamburg.

" Prof. Dr. R. W. S emon in Jena.

" Dir. August Siebert in Frankfurt a. 1.

"Hans Simon, Kaufmann in Stuttgart.

$"$ Dr. med. Stratz im Haag, Holland. 
Unser hentiger Bestand an Schlangen verteilt sich in folgender Weise auf Familien, Gattungen, Arten, Nummern und Stïcke $\left.{ }^{1}\right)$ :

\section{Schlangen.}

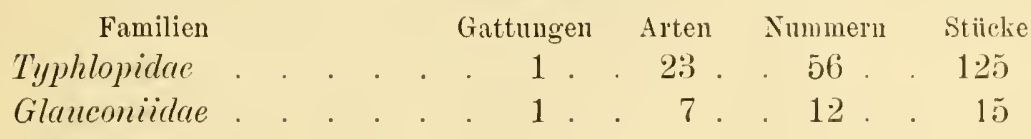

Boidce

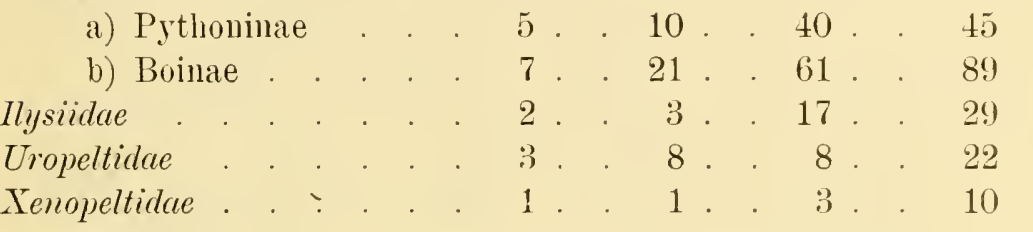

Colubridae

\begin{tabular}{|c|c|c|c|c|}
\hline a) Acrochordinae & 2 & 2 & 8 & 25 \\
\hline j) Colnbrinae & 79 & 285 & 818 & 1386 \\
\hline Rhachiodontinae & 1 & 1 & 5 & 13 \\
\hline Homalopsinae. & 4 & 8 & 36 & 65 \\
\hline Dipsadomorphinae & 37 & 84 & 298 & 461 \\
\hline Hydrophiinae. & 7 & 15 & 42 & 77 \\
\hline Elapinae & 18 & 51 & 124 & 168 \\
\hline ephalidae. & 3 & 9 & 18 & 30 \\
\hline
\end{tabular}

Amblycephat
Viperidae

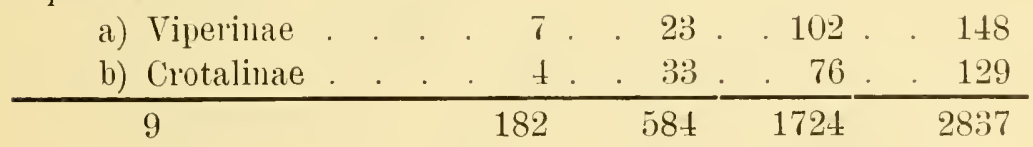

Vergleichen wir die Zahl unserer Schlangen (584) mit der' der im Jahre 1896 überhaupt bekannten Arten (1639), so besitzen wir also genan $35,63 \%$ davon. Wir sind also an Schlangen wesentlich reicher als an Eidechsen. Tor 55 Jahren hatten wir 150 Schlangenarten, jetzt 584, was eine Vermehrung unseres Bestandes um 434 Arten in diesem Zeitabschnitte bedentet.

Selır reich ist unsere Sammlung anch an Originalexemplaren, "Typen", die durch Reuß, Rüplell, Jau, Werner und den Verfasser s. Z. als neu beschrieben worden sind. In der folgenden Aufzählnug dieser 'Typen beschränke ich mich wie in

1) Wo in diesem Kataloge "Zahlr. Stiicke" steht, wurde in der folgenden Aufstellung stets nur die Zabl "sieben" gerechnet. 
dem I. 'Teile dieses Kataloges auf die Arten, deren Berechtignug allgemein anerkannt zı sein scheint, während ich die Namen der nenen Varietäten und die der in die Syrnonymie gefallenen Formen der geringeren Wichtigkeit wegen ïbergehe.

\section{Liste der in der Sammlung der}

Senckenbergischen naturorschenden Gesellschaft liegenden Schlangentypen.

1. Typhlops reuteri Boettger'. No. 7021 a. Nossibé. Zoolog. Anzeiger 1881 pag. 650.

2. Typhlops madagascariensis Bttg'1. No. 7025̃,1a. Nossibé. Abb. Senck. Nat. Ges. Bd. 11. 1877 pag. 3. 'Taf. 1, Fig. 1.

3. Typhlops ruber Bttgr. No.7031,1a. Insel Samar. Zoolog. Anzeiger 1897 pag, 164.

4. Typhlops mucronatus Bttgr. No. 7037,2 a. Nossibé. Ebenda 1880 pag. 279.

5. Typhlops congicus Bttgre. No. 7040a. Congo. Ebenda 1887 pag. 650.

6. Typhlops schinsi Bttgr. No. 7047,1a. Kalahari. Ber. Senck Nat. Ges. 1887 pag. 154, Taf. 5, Fig. 1.

7. Typhlops simoni (Bttgr.). No. 7051,1a. Syrien. Ebenda 1878-79 pag. 58 (Onychoceplealus).

8. Typhlops bisubocularis Bttgr. No. 7053a. Java. Zoolog. Anzeiger 1893 pag. 336.

9. Eryx thebaicus Reuß. No. 7125 c. Ober-Ägypten. Mus. Senck. Bd. 1, $183 \pm$ pag. 134.

10. Tropidonotus pmetirentris Bttgr'. No.7216a. Insel Halmahera. Zoolog. Anzeiger 1895 pag. 129.

11. Tropidonotus stumpffi (Bttg'1.). No. 7247a. Nossibé. Ebenda 1881 pag. 358 (Dromicus).

12. Tropidonotus halmahericus Bttgr. No.7264,1b. Insel Halmaliera. Ebenda 1895 pag. 130.

13. Helicops pictiventios Werner. No. 7275 a. Brasilien.

(Diagnose noch nicht rerïffentlicht).

14. Pararhadinaea melanogaster Bttgl. No. 7313,1a. Nossibé.

Diagnose s. pag. 33 nuter genannter Nummer.

15. Tetralepis fiuhstorferi Bttgr. No. 7320 a. Java.

29.32. Ber. Offenbach. Ver. f. Naturk. 1892 pag. 125. 
16. Gonionotophis iossi (Bttgr.). No. 7323,1 a. Kallerlu. Zool. Anzeiger 1892 pag. 318 (Gonionotus).

17. Znmenis bitaemialus Bttgr. No. 7392,1a. Guatemala. Diagnose s. pag. 4? unter genannter Nummer.

18. Zamenis mummifer (Renß). No. 7407 a. Ägypten.

Mus. Senck. Bd. 1, 183 t pag. 135 (Coluber).

19. Coluber mocllendorffi (Bttgr.). No. 805̌6,1 a. Süd-China.

Zool. Anzeiger 1886 pag. 520 (Cynophis).

20. Coluber schmackeri Bttgr. No. 8056,2a. Liukin-Inseln.

Ber. Offenbach. Ver. f. Naturk. 1895 pag. 108.

21. Rikadinace linkelimi Bttgr. No. 8178,1a. Nicaragua.

Diagnose s. pag. 68 unter genannter Nummer.

22. Fleischmamia obscura Bttgr. No. 8185̃a. Costa Rica.

Diagnose s. pag. 69 unter genannter Nummer.

23. Coronella amaliae (Bttgr.). No. 8193a. Marokko.

Zool. Anzeiger 1881 pag. 570 (Rhinechis).

24. Ablabes herminae Bttgr. No. 8277a. Iiukiu-Inseln.

Ebenda 1895 pag. 277.

25. Ablabes philippimus Bttgr. No. 8281 a und b. Philipuinen. Ebenda 1897 pag. 164.

26. Atractus lehmami Bttgr. No. 8310 a. Ecuador.

Diagnose s. pag. 80 unter genannter Nummer.

27. Atractus emmeli (Bttgr.). No. 8311,1a. Bolivia.

Ber. Senck. Nat. Ges. 1888 pag. 192. Fig. (Geoplis).

28. Calamorhabdium knekenthali Bttgr. No. 8330 a. Insel Batjan.

Diagnose s. pag. 82 unter genannter Nummer.

29. Calamaria semiammlata Bttgr. No. 8345 a. Bormeo.

Diagnose s. jag 84 unter genannter Nummer.

30. Hypsirhina alternams (Reuß). No. 9004,1 a. Java.

Ius. Senck. Bd. 1, 1834 pag. 155, Taf. 9, Fig. 3 (Brachyorhos).

31. Stenophis gramuliceps (Bttgr.). No. 9041 a. Nossibé.

Abh. Senck. Nat. Ges. Bd. 11. 1877 pag. 14, Taf. 1, Fig.3 (Dipsas gaimardi rar.).

32. Trabophis obtusus (Reuß). No. 9053a. Ägypten.

Irus. Senck. Bd. 1, $183 \pm$ pag. 137 (Coluber).

33. Hemivhagerhis lelleri Bttgr. No. 9119,1 a. Somaliland.

Zoolog. Anzeiger 1893 pag. 129.

34. Coelopeltis mö̈lensis (Reuß). No. 9143 c. Arabien.

Ius. Senck. Bil. 1, 1834 pag. 142, Taf. 7, Fig. 1 (C'oluber). 
35. Micrelaps muelleri Bttgr: No. 9249 a. Syricru.

Ber. Senck. Nat. Ges. 1879-80 pag. 137, Taf. 3, Fig. 2.

36. Diemenia maculiceps Bttgr. No. 9321a. Queensland.

Diagnose s. pag 116 unter genannter Nummer.

37. Hoplocephahus bitorquatus (Jan). No. 9349 a. Neusüiwales. Rev. et Jag. Zool. 1809 pag. 128 (Alecto).

38. Elapechis hessei (Bttgr.). No. 9360a. Congo.

Zool. Anzeiger 1887 pag. 651 (Elapsoidlea).

39. Elapechis boulengeri (Bttgr.). No. 9361 a. Sambesi.

Ebenda 1895 pag. 92 (Elapsoidea).

40. Amblycephalus moellendorffi(Bttgr ·). No.9443,1a. Süd-China. Ber. Offenbach. Ver. f. Naturk. 188 ó pag. 125 (Pareas).

41. Leptognathus peruana Bttgr. No. 9448 a. Per'u.

Diagnose s. pag. 128 unter genannter Nummer.

42. Lachesis nummifora (Rïppell). No. 9544,1 a. Mexiko.

Verz. Senck. Mns. Amphil. 1845 pag. 21 (Atropos).

43. Lachesis lutea (Bttgr.). No. 9553 a. Linkin-Inseln.

Ber. Offenbach. Ver. f. Naturk. 1895 pag. 111 (Trimeresurus).

Dem vorliegenden Kataloge wurden in Ammerkungen die Beschreibungen ron drei nenen Gattungen (Pararhadinaca, Fleischmannia und Calamorhabdium) und von je einer nenen Art vou Zamenis, Rhadinaea, Atractus, Calamaria, Diemenia und Leptognathus beigegeben. Die Diagnosen oder Fundortsangaben voll Stegonotus muelleri D. B., Zamenis mentorarius (D. B.) und florulentus (Geoffrr.), Atractus reticulatus Blgr. nnd Hemibungarus fasciatus (Pts.) konnten ebenfalls vervollständigt werden.

Um Wiederholungen zu verneiden, verweise ich betreffs der Konservierung, Aufstellung und Etikettierung und Numerier'ung der Reptilsammlung auf das, was ich $1892 \mathrm{im} \mathrm{„Katalog}$ der Batrachier-Sammlung:" pag. VII-IX der Vorrede gesagt habe.

Die Bestimmnng und Anordnumg geschah nach dem nenen Boulenger'schen System an der' Hand der Kataloge des British Mnseums:

"G. A. Boulenger, Catalogne of the Snakes in the British Husemun (Nat. Hist.). London: Vol. I, 1893; Vol. II, 1894; Vol. III, 1896. $8^{0}$." 
Auch bei dieser Gelegenheit möchte ich nicht verfehlen, meinen Dank auszusprechen für die fürsorgliche Unterstützung, die die Senckenbergische naturforschende Gesellschaft und die Herren Kustoden Adam und A ugust Koch in richtiger Würdigung der Verhältnisse der Vergrößerung und der Erhaltung besonders anch der Reptilsammlung widmen. Wie bisher ist der Sektionär für Herpetologie anch für die Folge bereit, die Bestimmung eingeschickter Reptilien und Batrachier - soweit es ihm seine Zeit gestattet - gegen Überlassung von Dubletten für uusere Sammlung zu ïbernehnen und den Einsendern sclnell und gern Nitteilungen ïber Namen, geographische Verbreitung, Giftigkeit oder Unschädlichkeit u. s. w. der vorgelegten Objekte zu machen. 

Ordintung

\section{()PHIDI}

\section{Familie I. Typhlopidae.}

Gemus 1. Typhlopss Schneid.

1. 'J'yplilops lineatus Boie.

Bonlenger. Cat. Smakes Brit. Mus. (X. H.) Tol. I, 1893 pag. Jj.

7nt a. Zahlr. Stïcke. Buitenzorge. West-dava. Ciescls. 1890 von Tr. Adolf Strubell. Bonn.

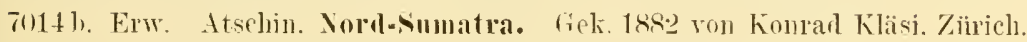

2. Typlilops braminus (Daud.).

Bonlenger, lat. I pag. 16; Boettger. Ahl. Senck. Gres. Br. 11. 1879 pag. 45!). Thaf. 1. Fjo. 1 und \%ool. Anzeiger 1882 pat. 479 (T. euproctus).

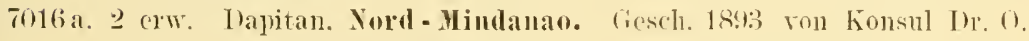

Fr. von Moellendorff. Manila.

70t6h. Erw. Insel Termate. Erh. 16!) von del Riuppellreise Prof. In. W. Kiikentlials, J'nil.

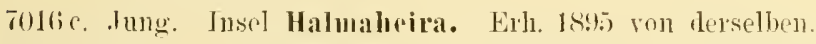

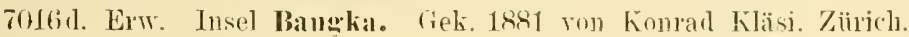

7olde. Erw. Gebirge Lo-fon-shan bei Kanton. Siid-('hina. Gek, 1886 von Otto Herz. St.-Petershurg.

$7016 \mathrm{f}$. Zahlr. Stiiche. Madras. Gesch. 188! ron Theodor Koll, daselhst.

7016g. Erw. Insel (Cobí, Philippinen. (Gesch. 1890 von (Otto Koch, rlaselbst. 7016lı. Zahlr: Stürke. Manila. Iszou. (ipselı. 1890 ron Konsul 1). O. Fr. ron Xoellendorff. dasellsst.

7016i. Erw. Mimlanao. (ieseln, 1888 von demselben.

7016k. Erw. Nigiris. Niid-Iudien. Gesch. 1890 rom Insemm in Marlas.

70161. Erw. Caplon. liesels. 184ti von W. Worms.

7016m. Erw. Insel Hainan. Siidl- Cluina. Grek. 1886; ron Otto Herz, St.F'etershure.

7016n. Gerw. Bnitenzorg. West-J a ral. Ciesch. 1890 von Dr. Adolf Strubell. Bomn. 70160. Erw. Atschin. Nom-Sumatra. Ciek. 1882 yon Konrar Klïsi. Ziirieh.

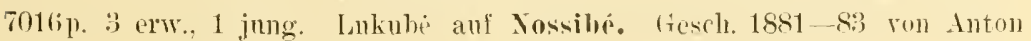
stumpff, daselbst. 
7016i. Halbw. (Typus ron T. enproctus Btgr.) Ehendaher. (iesch. 1882 ron demselhen.

70161. 2 erw. Ambatolampy. Lembatokahai. West-Madaysuskr. Cieseh. 1897 von Dr. Alfred Voeltzkow. Borlin.

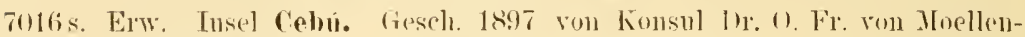
dorff. Manila, mud (Otto Koch. ('obí.

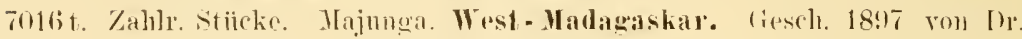
1. Vooltzkow.

70161. Erw. Ietsako. X.- Majunga. West-Madlagaskar. (ieseh. 1897 rou demselhen.

ral. l'a m meces Gthr.

G ii ut her. Rept. Brit. Tndia pag. 176. Taf. 16. Fig. C' (tenuis).

7o16.1 a. Halbw. Madras. Gesch. 1889) ron Theodor Kollb, dasellst.

\section{3. 'Typhlops reuteri Btgr.}

Boct ger, \%ool. Anzeiger 1881 pag. (i.) (nächstremwanlt $T$. como.

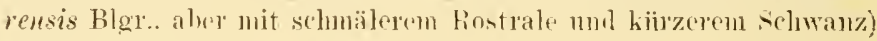
und 188. pag. tor (T. lenzi).

7021 a. Enw. Lukubé auf Nossihé. Gesclı. 1881 ron Anton stumplff. dascllost.

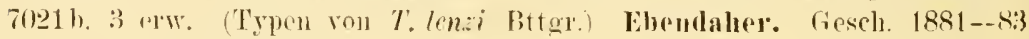
ron remselhom.

\section{Typhlops vermicularis Merr.}

Boulenger, Cat. I pag. 21: Boettgor. Zowl. Jahrb. Bil. 3. 1888 pag. 920.

7022a. Erw. Prevesa. Epirms. Gesch. 1892 ron ('ésar ('onéménos. rlaselhst. 7022 b. Erw. lusel Enböa. Gesch. 1880 von frl. Josephline Thicsse. ('lalkis. 7022 e. Erw. Xenochori. Norll-Euhïa. Geseh. 1891 rom Fr. de Mimont. daselhst. 7022 d. Halbw. (Riiplell. ('at. II EEE⿱3а) Nisib. Inatolien. Fescl. 1840 ron In. Schneider. hicr.

702.2 e. fi Ste. Jermsalem. Fesels. 1881 von Hans Nimnon. Stuttgart.

5. Typhlous muelleri Schlegel.

Bonlenger. Cat. I pag. 2 5.

7025a. Erw. Reich Deli, Nord-Sumatra. Gesch. 188 fon Clemens Hohwiesner: hier.

6. Typhlops madagascariensis Bttgr.

Boulenger, (at. I pag. 25; Bocttgel. Abh. Senck. Nat. lies. Br. 11. 1877 pag. 3, Taf. 1, Fig. 1.

7025,1 a, Erw. Nossibé (Marlagaskar). (resch. 1875 ron Konsul (. Ej)enan. Hamburg. 
7. Typhlopis flavirenter les.

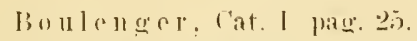

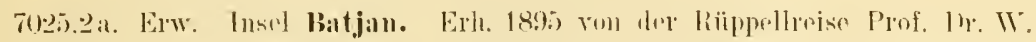
Kiikenthals, . Trin.

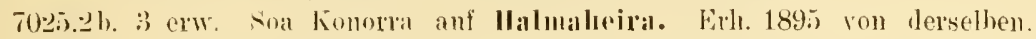

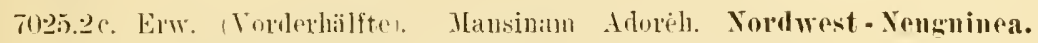
Gesch. 1890 von 1)r. Adolf struhell. Bonm.

8. 'Typhlops reticulatus (T.).

boulenger. l'at. I pag. 27 .

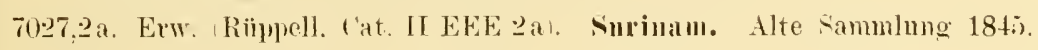

Val. crassa Jall.

J a n, Icon. Ophrid. Lief. 5, Taf. 5, Fig. 7.

7027.3 a. 르. (Rïppell, Cat. II EEE 1a). Sïd-Imerika. Get. 1841 rom Taturlist. Inseum in 1)armstailt.

var. troscheli Tall.

J a n, Icon. Ophid. Lief. t. Taf. 6. Fig. 1c.

7028 a. Erw. Trinidad, Westindlien. Fesch. 1888 rou Ingenienr G. Gerold, Port of Spain.

9. Typhlops lombricalis (L.).

Bonlenger. Cat. I pag. 31.

7081 a. Erw. Siid-Amerika. Get. 1882 non Naturh. Mnsemu in Lübeck.

7081 b. Halbw. Cap Haytien, Haiti. Fek. 1888 rom Naturh. Institut Linnaea, Berlin.

10. 'Typlilops lubel' Bttgr'.

Boettger, Zoolog. Anzeiger 1897 pag. 164.

7031.1 a. Erw. Insel Samar, Philippinen. Gesch. 1897 ron Konsul Tr. O. Fr. von Moellendorff. Manila, und otto Koch. Cehú.

11. 'Typhlops polygrammicus Schlg.

Bonlenger. Cat. I pag. 34: Ian. Icon. Ophid, pag. 14, lief. 9, Taf. 1. Fig. ㄴ (T. rueplelli).

7034.2a. + erw. (Typen von T. rueppelli . Tan). Sriney, Sensidwales. Gesch. 186 e von Aram Becker. dasellost. 
12. Typlolops wiedi Pts.

Bonlenger. C'at. I pas. 36 .

7036 a. Erw. Burnett Tiver. Quecusland. Gesch. 1894 ron Prof. Jlr. IR. WT. Semon. Jena.

13. Typlilops mucronatus Jitgor.

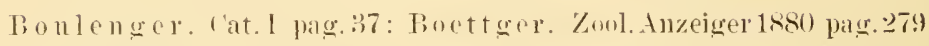
mot the renck. Nat. fies. Brl.12. 1881 pag. 4:38. Taf. 1. Fig. 1.

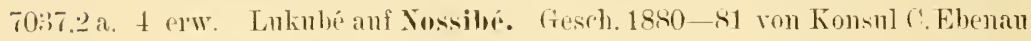
und Auton stmmpff, dasellst.

14. 'Typhlops boettgeri Blgr'.

Bo 011 lenger. ('at. I pag. 39. 'Taf. 2. Fig. ti.

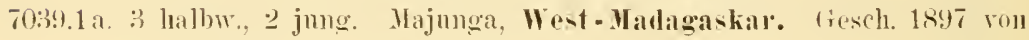
Dr. Alfred Voeltzkow. Berlin.

15. Typhlops cungicus Bttgr.

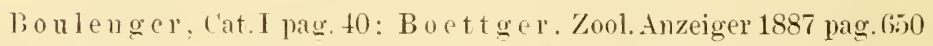
und Ber. Fenck. Nat. Ges. 1888 pag. 4t, Taf. 1. Fig. 5.

7040 a. Erw. Povo Netonna bei Banana Inter-('ungo. Gek. 1886 ron Panl Hesse. Venedigr.

16. Typlilows lunctatus (Leach).

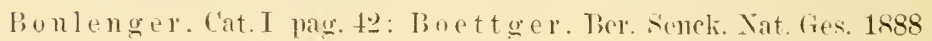
pag. 4. (T. eschrichti).

7042. a. Erw. (var. Ac Blgr.). Gollkiiste, Gronze gegen Aschantiland. Fresch. 1884 ron Hans vimon. Stuttgart.

rar. intermedia .Tan.

Boule nger. l. c. pag. $4:$.

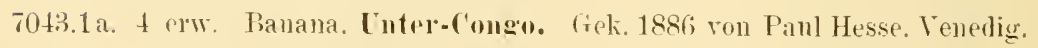

var. nigrolineata Hallow.

Boulenger l. c. pag. 43.

7043,2a. 2 erw. Kannerun. (iek. 1897 von 1)r. Georo Kraatz. Berlin. 
17. 'Typhlops delalande i Schlg.

Boulenger. ('at. I pag. ti).

Var. pall cisqliamata Bttgr.

Boettger. 22.|23. Ber. Offenbach. Ter. f. Natnrk. 1883 pag. 1õ.

70tsa. 3 erw. (mit 26 Schuppenreihen um die liumpfmitte). smitlutielıl.

Transvaal. Gescls. 1882 ron Hans ,imon. Stuttgart.

18. Typhlops dinga (Pts.).

Boulenger, Cat. I pag. tis.

7045.1a. Halbw. Boroma am Nambesi. (iesch. $189 t$ ron Prof. Dr. Osk. Boettger, hier.

19. Typhlops mucluso (Pts.).

Boulenger. lat. I pag. 4 ti.

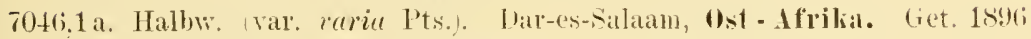
rom Naturh. MLusenu in Berlin.

20. 'Typhlops schinzi Bttgr.

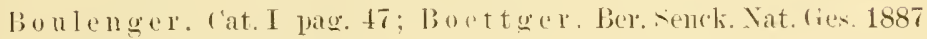
pag. 1ot, Taf. j. Jị. 1.

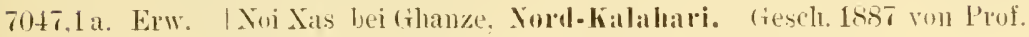
Ir. Hans selinz. Zïrich.

21. Typhlolis simoni (Bttgro).

Banlenger. Cat. I pag. 51: Boettger. Ber. senck. Nat. Cres. 1878 -79) pag. 88 mu $1680-81$ pas. 135\%. Taf.3. Fig. 1 (Onychocephalus).

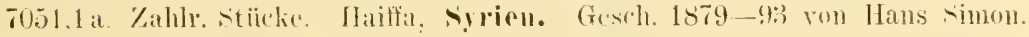
sintterart.

22. Typhlops bistbocelaris Bttgr.

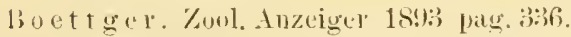

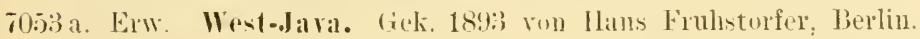

23. 'Typhlops at er' Schlg.

Boulenger, ciat. I page.jis.

7)B3.1 a. \& erw. Foa Konorra anf Halualheira. Erh. 1895 ron der liappellreise Prof. Mr. W. Külichthals, Iena. 


\section{Familie II. Glauconiidae.}

\section{Genus I. Glauconia Gray.}

1. Gla uconia distanti Blgr.

Bonlenger. Cat. 1 pag. 62.

706ㄹ. Er. Boroma an Sambesi. Gesch. 1895 ron Prof. Dr. O. Boettger. hier.

2. Glaucunia albifrous (ITgl.).

Boulenger. Cat. I pag. (ii3.

7063 a. Erw. Tenezuela. Gesch. 1893 ron Lr. Heinr. Lenz, Lübeck.

7063 b.c. Erw, nud jung. Trinidad. Fresch, 1894 ron F. W. Trich und R. R. Mole. Port of spain.

7063 d. Erw. rar. albipuncta Burm.). Bnenos Aires, Argentina. Gek. 1877 ron Pïhl. Hamburg.

7063 e. 2 erw. (var. albipunctu Burm.) (njapa. staat Matogrosso, Brasilien. liesch. 1886 von Jakol schumacher: hier.

7063 f. Erw. Mapiri, Kebentuss des Beni. Bolivia. Gesch, 1888 ron Ferdinand Emmel, Arequipi.

7063 g. Erw. Santa Ana. Prov. Inzco. Perll. Ciesch. 1890 ron Friedrich kimmel. Gelnhansen.

3. Vilauconia conjuncta (Jau).

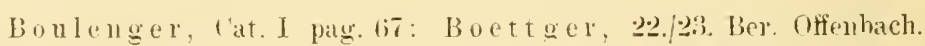

Ter. f. Naturk. $188: 3$ pag. 1ini (Stenostoma nigricans).

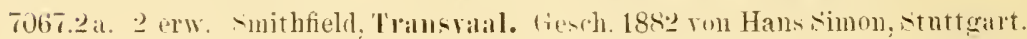

4. Cilaucunia seutifrous (Pts.).

Boulenger, cat. I pay. lise.

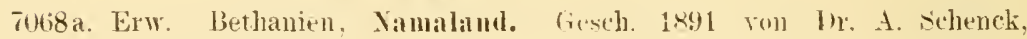
Halle (Siale)

5. Granconia sullevalli (Jall).

Bull lenger, ('at. 1 pag. (is'.

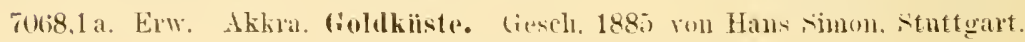

6. (ilallionia biculer (.Jall).

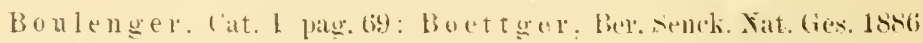
pag. 29.

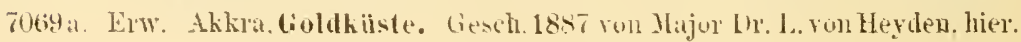

7. Glaucunia macrolepis (Pts.).

Bunleuger. Lat. 1 pay. $6 !$.

70ti9.1a. Erw. san kstehan hei Puerto tabello. Venezmela. Gesch. 189j vou Kunsul fr. Hather. danelhet. 


\section{Familie III. Boidae.}

\section{Subtamilie T. Pythominne.}

Genus I. Nardoa Gray.

1. Nardoa boa (Schlg.).

Boulenger. Cat. I pag. 7fi.

7076a. 2 halbw. Mioko, Nenirlaud. Gek. 1887 von Pöhl. Hamburg.

Gemus II. Liasis Giay.

1. Liasis childreini Gray.

Boulenger. Cat. I pag. 7т. Taf. t, Fig. 1.

7077a. Erw. Baudin Island, Nord-Australien. Get. 189:3 rom British Juseum ( $\mathrm{Y} . \mathrm{H}$.$) . Lomlon.$

Genus III. Python Daud.

1. Python spilotes (Lacepo).

Bо leuger: ('at. I page. 8?.

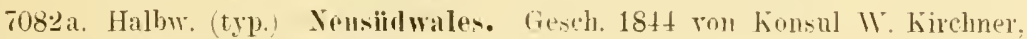
Wiestraden.

7082h. Hallow. typ.: Rüppell. ('at. IJI E :3a als P. punctretus). Ehendaher. fiet, 1840 rom Hofmuseum in IVien.

708: e. Erw. (typ.) Nenholland. Gesch. 184.2 ron der Tenen Zoolug. Gesellschaft. hier.

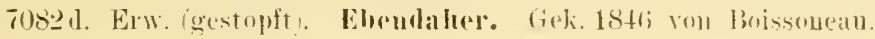

val. B (rariegata kretit).

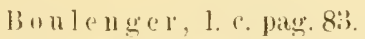

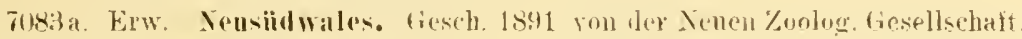
hier.

rar. rasiegata livay.

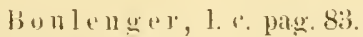

7083.1 a. Halbw, Burlett liver. Quepuslaml. Gesch. 1894 vom Prof. Wr. K.

II. Semon. dena.

2. Pythun amethystinus (schmi.).

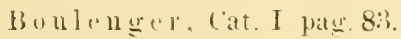

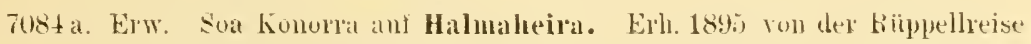
Prof. 1'r. W. Külsentlals, Jena.

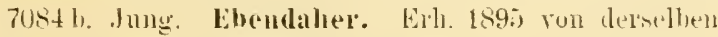

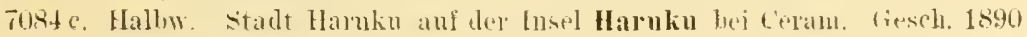
rou [nr. Adolf strubell. Bomn. 


\section{Python reticulatus (Schnd.).}

Boulenger. Cat. I pag. 85.

7085a. Halbw. Reich Deh, Murd-Sumatra. Gesch. $189 t$ ron Wwe. H. Krämer, hier.

7085 h. Jung u. ein Kopf. Insel Batjan. Erh. 1895 ron der Rüppellreise Prof. Dr'. W. Küikenthals, .Tena.

7085 с. Halbw. Rïppell, (at. III E 2 c als P. schneideri). Jara. Get. 1838 rom Rijksmusem in Leiden.

7085) (1. Halbw. Atschin. Yord-Smmatra. Gek. 1882 ron Konrad Kläsi. Zuirich. 7085 e. Halbw. Riippell, (at. III E2 2 als P. schneideri). Java. Gesch. 1838 von Dr. von siebold, Leiden.

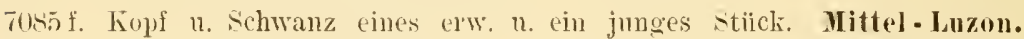
Gesch, 1888 von Konsnl Dr. O. Fr. von Moellentorff, Manila.

708.) galhw. Thai anf Hitn. Yord-Amboina. Gesch. 1890 roli ne. Adolf Struliell. Bomm.

7085 h. Halbw. Insel Mindw'o. Get. 1889 von B. schmacker. Shanghai.

7085) i. hallw. Reich Deli. Mord-Numatri. Gesch. 1SS6 von 'lemens Hohwiesner', hier.

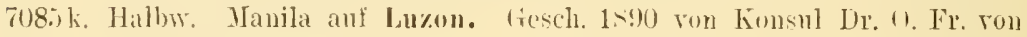
Ifoellendorffi, dasellost.

7085l. Erw. Singapore. Gek. List ron Hambure.

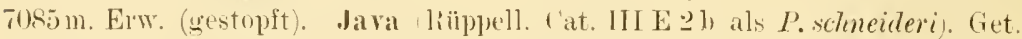
1832 rom Rijksmuseum in Lejden.

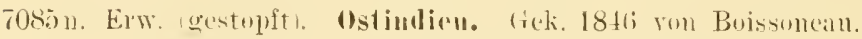

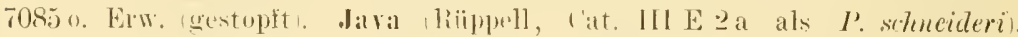
(iesch. $18: 38$ ron Dr. men. Dölx.1. Bataria.

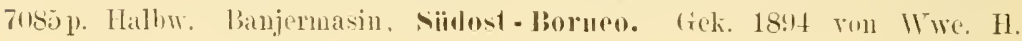
Krieh, lier.

$$
\text { 4. Pytholl sebate (Gmel.). }
$$

Bonlenger. (at. I pag. Sti.

7086a. Halhw. Akkra. Ginllkiiste. Gesch. 188 ron Major Dr. L. rom Heyilen. hier.

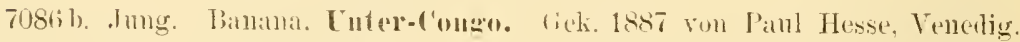

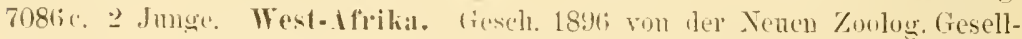
schatt, licer.

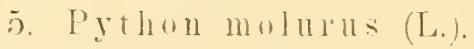

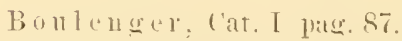

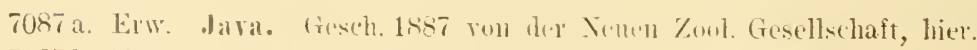

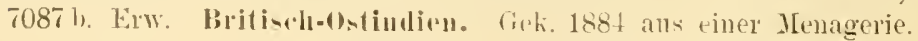

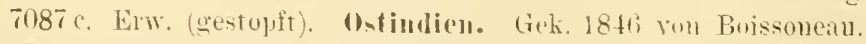

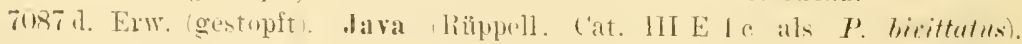

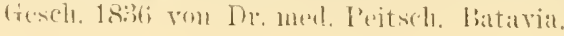


7087 e und g. 2 erw. (gestopft). Jara (Rüppell, Cat. III E 1 a-b als $P$. birittatus). Gesch. 1838 ron Dr. med. Döbel, Batavia.

7087f. Erw. (gestopft). Jara. Gek. 1884 aus einer Menagerie, hier.

7087 h. Erw. (gestopft). Ostindien. Gek. 1846 ron Boissoneau.

6. Python regius (Shaw).

Bo ulenger, Cat. I pag. 88.

7089 a. Halbw. West-Afrika. Gek. 187 r von Hamburg.

Genus IV. Chondropython A. B. Mey.

1. Chondropython viridis (Schlg.).

Boulenger: Cat. I pag. 90; Boettger in Semon, Zool. Forsch. Bd. ว, pag. 120, Taf. 5. Fig.. 3 (1894).

70!90a. Halbw. Siidost-Neuguinea. Gesch. 1894 ron Prof. Dr. R. W. Semon. Jena.

\section{Gemus T. Aspridites Pts.}

1. Aspirites melanocephalus (Kreftit).

Boulenger. lat. I pag. 91: Boettger 1. c. pag. 119.

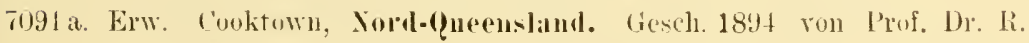

IV. Semon, . Iena.

\section{Subfermilie :. Boinere.}

Cienus II. Evicintes Wigl.

1. Euprates cenchris (L.).

B

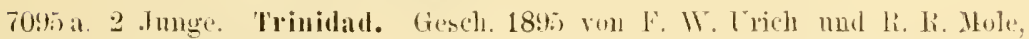
Port of spain.

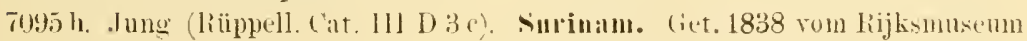
in Leiden.

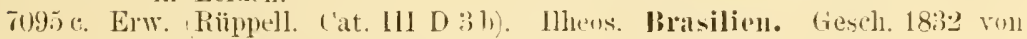
Gelor. Koclo. hiter.

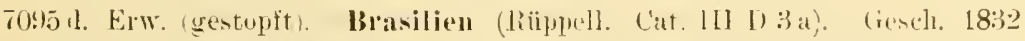
ron denselbents.

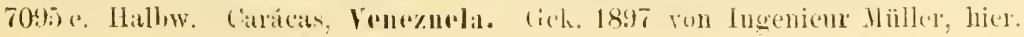


var. fusca Gray.

Boule llger, Cat. I pag. 96.

7096 a. Halbw. Trinidad. Gesch. 1893 von F. W. Crich. Port of Spain. 7096 b. Halbw. (Rüppell, Cat. III D 3 d). Venezuela. Alte Sammlung 1845. 7096 c. Erw. Trinidad. Gesch. 1896 ron F. W. Urich und R. R. Mole, Port of Spain.

2. Epicrates striatus (Kischer). B o u lenger. Cat. I pag. 96.

7096,2 a. Erw. San Doningo. Gek. 1877 ron Hamburg.

\section{Genus VII. Corallus Daud.}

1. Corallus cookei Gray.

Bonlenger', ('at. I pag. 99. 'Taf. 4, Fig. 3.

7099a. Erw. (typ.) Insel Greuada. Gesch. 1893 ron F. W. Grich and R. R. Mole. Port of Spain.

7099 h. Jung ityp.) Trinidad. Gesch. 1895 ron denselbell.

7099 c. Erw. typ.) Ehendaher. Gesch. 1995 ron denselben.

vall: ('.

boulenger. I. '‘ jag. 100.

7100 a. Erw. Grenada. Giesch. 1895 ron densellen.

7100 h. Erw, Ebendaher. Gesch. 1895 von denselbern.

vall lusthenbergi ('opr.

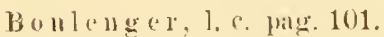

7101:. Enw. Trindad. Geseh. 189:3 von denselben.

2. Colallus hortulanus (L.).

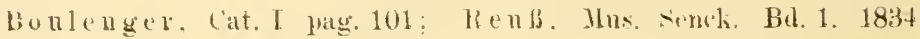
parg. 129 (Bou modesta).

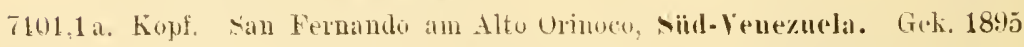
ron Georg Hübner. Dresden.

7101.1b. Hallw. Typus von Bou modesta kiss.) Llheos. Brasilien Rüppell. ('at. LIL D 5 h. Gesch. 1835 ron Gebl: Koch. bier.

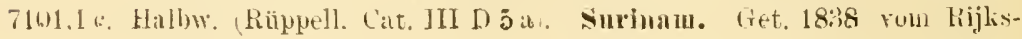
muscun in Leideu. 
3. Corallus caninus (L.).

B o ulenger, Cat. I pag. 102.

7102.1 a. Halbw. (Rüppell, Cat. III D 4 a). Brasilieu. Alte Sammlung 1845 .

7102,1 b. Jung. Unterer Rio Madeira. Gek. 1896 ron Georg Hübner, Dresden.

4. Corallus madagascariensis (D. B.).

B ou lenger, Cat. I pag. 103; B o et tger, Abh. Senck. Nat. Ges.

Bd. 11, 1877 pag. 21 und Bd. 11, 1879 pag. 470.

7103 a. 3 halbw. Nossibé. Gesch. 1879-81 von Konsul C. Ebenau und Anton Stumpfi, daselbst.

\section{Genus VIII. Enygrus Wgl.}

1. Enygrus australis (Montr.).

Boulenger, ('at. I pag. 10:).

7105а. Halbw. Nenhehriden. Get. 1893 vonn Brit. Yusenm (X. H.) in London.

2. Enygrus bibroni Hombr. Jacy.

Boulenger, Cat. I pas. 10\%.

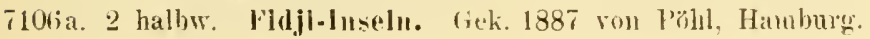

3. Enygrus cariuatus (schmd.).

boulenger, cat. I jay. 10\%.

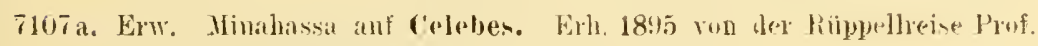
Di: II: Külsentlials, Jentit.

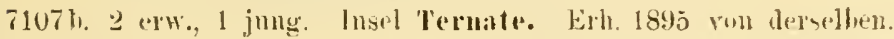

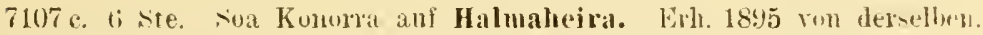

7107 d. Erw. nnd :3 jung. Insel Batjan. Erlt, 18!5 von dersellyen.

7107е. Hallw. Palau-Luseln. Geli. 188 r ron P’öhl. Hamburw.

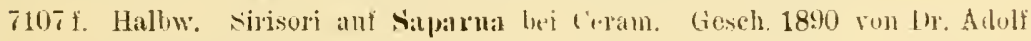
Strulbell, Bom.

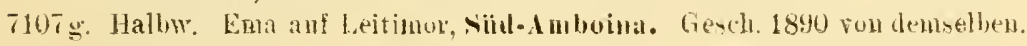

\section{Eufgrus asper (Gthr.).}

Bunlenyer. Cat. I pay. 109.

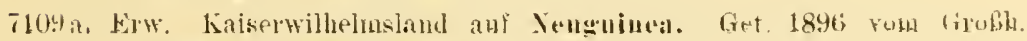
Jusemn in karlsitule. 
Genus IX. Ungalia Gra!y.

1. Ungalia maculata (Bibr.).

B oulenger, Cat. I pag. 112.

7112a. Halbw. Haiti. Gek. 1877 von Hamlurg.

Genus X. Eunectes IVgl.

1. Eunectes murinus (L.).

Boulenger. Cat. I pag. 115.

7115 a. Erw. (gestopft). Surinau (Rüppell, ('at. III D 2a). Gek. $184 t$ ron Cardua.

\section{Genus XI. Boa L.}

1. Boa constrictor L.

Boulenger. ('at. I pag. 117.

7117a und c. Jung u. erw. Trinidat. Gesch 1893 u. 95 ron F. W. Urich und R. R. Mole. Port of spain.

7117\%. Erw. Ehendaher. Gesch. 1876 rom l'arl L'rich, l'ort of spain.

7117 d. Halbw. Niid-lmeriki. (iek. 1883 ron H. Deermann, hier.

7117 e. :3 Embryomen. Fluss tio Amaro, taat Bahia, Brasilien. Gesch. 1871 ron Alugust frommel. hier.

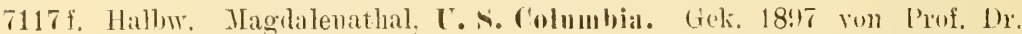
Fritz liegel. Iena.

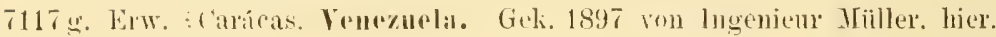

2. Bua diviniloqua (Lam:).

Boulenger, l'at, l pag. 118.

7118.1 a. Erw. Westindirn. Giek. 187 rom Hamburg.

: P. Par imperator Daud.

lionlengere l'at. I pag. 119)

7119:. Jung. Retalhnlen, Guatemalis. Gesch. 1894 ron Carl Fleischmam, Gillatemala.

711! b. Em, Maxiko. Gek. 1884 ans ciner Mentgerie. hier.

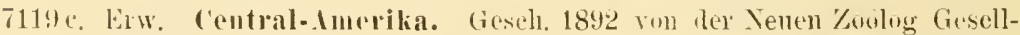
sehatit. liser.

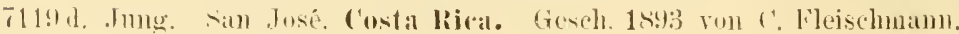

$$
\text { 4. Bua maragaxcariensis (D. B.). }
$$

Bunlenger, l'at. I pag. 120): Boettger. Abl. Yenck. Nat. Ges. Bd. 11, 1877 pag. 20 .

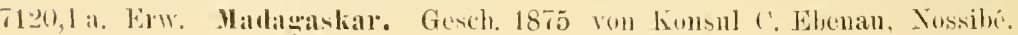


7120.1\%, 2 erw. Kamlani im Sïden der Bembatokabai. West-Madanaskar. Geseh. 1892 ron l'r. Alfred Voeltzkow, Berlin.

7120.1 е. Krw. Majungi, West-Malaziskar. Gesch. 1892 von demselben.

\section{Genus XII. Firy.x Dand.}

1. Elyx coniens (Schmo.).

boulenger, l'at. 1 jag. 124 .

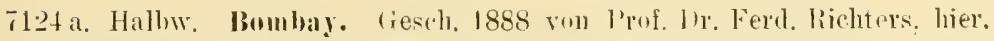

7124b. Halbw. (gestopft). Ostindion. Gok, 18ti rom Boissoneatl.

2. Fryx thebaicus Reuf.

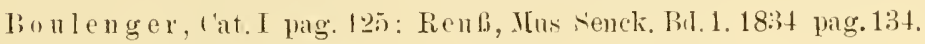

7125a. Halhw. Oher-legypten. Crek. 187 ron l'ühl. Hamburg.

7125 b. 2 erw. Thebers. Iegyten. Gols. $1880 \mathrm{rm}$ Gistar Selmeiler. liasel.

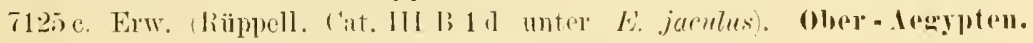

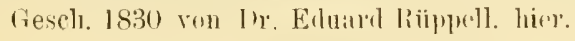

8. Exyx jaculus (L.).

Bonlenger, ('at. I pags. 125: lioettger. Ber. Senck. Nat. Ges. 1879-81) pag. 166 mil in Radile, Fum. Casp.-Geb. 1886 pag. 73.

7126 a. 3 Ste. Salmas, Persien. Gesch. 189; vom Pforrer G. Nägele. Waltersweier bei (offenburge (Barlen).

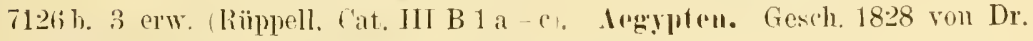
Elnard Riippell. hier.

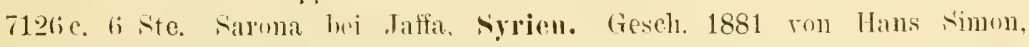
Stuttgart.

7126،. Erw. Transkispien. Gesch, 1897 vou der Neuen Zoolog. Gesellschaft, hier.

var. milialis l'allas.

Boetteger. Zool. .lahıb. Brl. :3, 1888 pag. 938.

7127a. 2 erw. Askhahar, Transkispirn. Gesch. 1888-90 ron C. Eylandt, daselbst.

7127b. Erw. Uor-kala am kintselik, Transkilspien. Gesch. 1888 ron Staatsrat Dr. linstar ron Radle, Excz., Tiflis.

4. Eryx johni (Russell).

Boulenger, ('at. I pag. 127.

7127.1 a. Erw. Simd. Gesch. 1890 rom G. A. Bonlenger. F. K. S.. Lomdon.

5. Eryx elegans (Grar).

Boulenger. Cat. I pag. 128, Taf. 5. Fig. 1.

7128 a. Erw. ․ Ai-Dére, Transkaspien. Gesch. 1894 ron staatsrat Dr. Gustar ron Radile, Excz., Tiflis. 


\section{Familie IV. Ilysiidae.}

\section{Genus I. Il!ysirt. Hempr.}

1. Ilysia scytale (L.).

Bonlenger, ('at. I pag, 133.

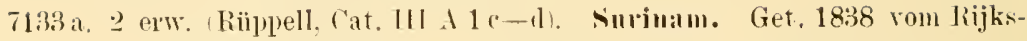
mnsemm in leiden.

7133\%. Evw. (Riippell. (at. III I $1 \mathrm{~g}$ ). Niid-Amerika. Alte Sammlıng 1845. 7133 c. \& elw. (Rïppell. Cat, III \& 1 a-b, e-f). Surhuim. Gesch. 1839 ron Dr. Sichel, hier.

7138 d. Halbw. (Rïppell. Cat. III A 3 a). Niid-lurerika. Alte Sammlung 1845.

\section{Gemes II. Cylindrophis Wgl.}

1. Cylindrophis rufus (Laur.).

Boulenger. Cat. I prag. 1:35.

713isa. Halbw. Pontianak, West-Borneo. (resch. 1893 ron Baron Alhert von Reinach, lier.

71356. Erw, stahat in Deli, Nordost-simatral. Gesch. 1893 ron Fr. Berschlag. daselbst.

7185e. Jung. BaramthB, Nord-Bormen. Erls. 1895 von der Riippellepise Prof. Dr. W. Kiikenthals, Jena.

7135 d. Erw. Lusel Batjan. Erlı. 1895 rou lerselben.

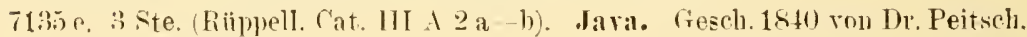
Bataria.

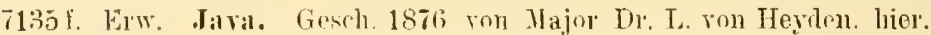

7135 g. Erw, 1l. jung. Buitenzong. West.Jara. Gesch. 1890 ron Dr. Arlolf Strubell, Bonn.

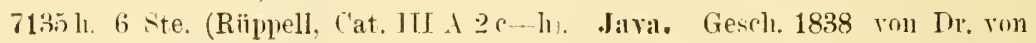
Siebold, Leiden.

7135i. Halluw. Bangkuk, Nian. Gesch. 188; ron Konsul Dr. Fr. O, ron Ifoellendorff, Yanila.

$7135 \mathrm{k}$. Halhw. Snltanat Deli. Norlout-sumatra. Gesch. 1891 ron Dr. merl. O. Benecke, daselhst.

71351. Halbw. Bornes. Gescli. 1886 ron F'rof. Dr. O. Boettger', hier.

7135 m. Erw. Jara. Geseh. 1896 ron Direlitor August Siehert. hier.

2. ('ylindrophis maculat tus (L.).

Bonlenger. Cat. I pag. 136.

7136ia. Erw. Ceylon. Gek. 1889) von Hans Frubstorfer, Berlin. 


\section{Familie V. Uropeltidae.}

\section{Genlle I. Lihinophis Hemm!.}

1. Rhinophis oxyruhyelus (schnd.).

Boulenger. I'at. I jag. 1 1.

7140 a. Frw. Central-('eylon. liek. 1889 von Hans Fruhstorfer, Berlin.

2. lihinophis planiceps Pts.

Boulenger' . ('at. I pag. 141 .

7141.1 a. Erw. C'entral-f'rẹlon. Get. 1889 rom Xaturlı. Musenu in Basel.

3. Rhimoplis trevelyamus (Kel.).

Bonlenger, ('at. l pag. 142.

71t2 a. Frw. Central-Ceylon. Get. 1889 rom Naturl. Mrusemm in Basel.

4. Rhinophis hythi Kel.

Boulenger. lat. I jag. 14 .

7144 a, 3 erw. 2 jumg. ('entral-Ceylou. Gesch.18t6; ion W. Worms, daselhst.

Gemus II. Silybura Gra!y.

1. Silybura pulueyensis (Bedd.).

Bonlenger, ('at. I pag. 147; Buettger, Ber', lffenbach, Ver. f. Naturk. 189? pag. 76.

7147a. 6 Ste. Kodaikanal. Panni Jlills, Siid-Indien. Gesch. 1889 von

Theodor Kolb, Mardras.

2. Silybura nigla Bedrl.

Boulenger. Cat. I pag. 151.

7151 a Erw. J. Kodaikanal, Palni Hills, Niid-Indien. Gesch. 1889 ron

Theodor Kolb, Madras.

3. Silybura breris Gollur.

Bonlenger. Cat. 1 pag. 158.

7158a. Erw. Nilgherries, Niid-Iudirn. Gesch. 1890 vom Musenu in Madras.

\section{Genus III. Patyplectrums Gthr.}

1. Platrplectrurus madurensis Berd.

Boulenger. (at. I pag. 166, Taf, 11. Fig. 2.

7166a, 6 Ste. Kodaikanal, Palni Hills, Siid-Indien. Gesch. 1889 ron Theodor Kolb, Mardras. 


\section{Familie VI. Xenopeltidae.}

\section{Gems. 1. Lenopeltis Reinu.}

1. Xenopeltis unicolor Reinw.

Bonlenger, ('at. T pag. 168.

7168 a. Erw. n. 4 .tnnge. Buitenzorg, Want-Jali. Gesch. 1890 ron Dr. Adolf strubell. Bonn.

71li8b. 4 Ste. Atschin. Norl-sumalral. Gek. 188-2 ron Konral Kiäsi. Zürich. 7168 e. Erw. Insel Bangkil. Gek. 1881 run demsellen.

\section{Familie VII. Colubridae.}

\section{sulbermilie 1. Areorhordimese.}

Gemms I. Acrochordus Hormst.

1. Acrochuldus javanicus Hornst.

Bonlenger, ('at. I llag: 17:).

7173 a, Kolıf. Buitemzorg, West-Java. Gesch, 1890 ron Dr, Arlolf Strubell. Bomn.

$717: 3$ b und c. Elw. (gestopft). Jara (Riippell, Cat. III F 1 a-b). Gesch, 1838 ron Trr. med. Döhel, Batavia.

Gemu: II. Chersydins Cur.

1. ('hersydrus gramulatus (Schnd.).

Bonlenger. liat. I pag. 17 t.

Flita. G elw. Janila anf Lnyon. Gesch. 1888 ron Konsul Lr. O. Fr, rom Mroellendorff, daselhst.

717th. Zahlr. Ste. Mittel-Luzon. Gesch. 1888 ron demselben.

7174 C. Zahlr, Ste. Tmgebung von Manili. Gesch. 1891 ron demselhen.

$717+$ d. Jung. See Taal, Luzon. Gesch 1897 ron denselben.

717te. Halhw. Nimsilnore. Gouch 1892 ron Plof. Dr. O. Bowtger. hier.

\section{Subfomilie :e. Colubrimar.}

Genus I. Polyodontophis Blgr.

1. Polyodontophis torguatus (Blgr.).

Boulenger. ' at. I pag. 18:3; Boettgor, Alh. Senck. Nat. Ges.

Bd. 11. 1877 pag. 8 (Enirognathus rhodogaster).

7183 a. \%alılr. Ste, Tossilur. Gesch. 1879-83 von Konsul ('. Ebenau und Anton Stumpfif, daselhst.

7183 b. Halbw. n. 2 Junge. Whendaher, Gesch. 1897 ron Dr, Alfred Toeltzkow, Berlin. 
2. Polyoduntophis collaris (rray).

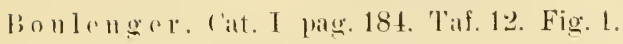

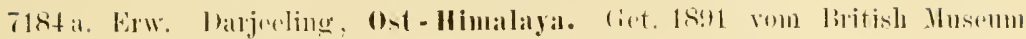
(.). 11.). I Lomblon.

:) Polyodontophis geminatus (Boie).

Bonlenger. C'at. 1 pay. 185; boottger. Pier. offenbach. Ver. f. Naturk. 1892 pag. 127 (melanocephalus.s.

7185 a. Erw, (trp.). Buitenzorer. West-Java. Gesch. 1890 ron Dr. Arolf Strulkell. Bomm.

7185). Elw. (typ). Tenggergelirge in 1200 m. Ost-Java. Gek. 1891 ron Hans Frubstorter. Berlin.

4. Polyodontophis bivittatus Blgr.

Bonlenger, C'at. III pag. כ̆97.

7185.ta. Erw. Insel Culion, Calamianes, Philippinen, Gesch. 1897 ron Konsul lu. (). Fr. ron Moellendorff. Manila.

5. Polyodontophis subpunctatus (U. B.).

Boulenger', C'at. I pag. 186.

7186a. Erw. Honore, Britiseh Ostindirn. Geseh. 186t ron Missionar Alhrerht. daselhst.

\section{Gemus II. Dromicodryas Blgr.}

1. Dromicodryas bernieri (D. B.).

Boulenger, (at. l pag. 189); lioettger, Abl. Senck. Nat. Ges. 33. 12. 1881 pag. 44:3 (Herpetodryas bernieri var. trilineata).

7189,1 a. Erw. (Typus von 11. bemieri var. trilineata Bttgr.). Nossibri. Gesch. 1876 ron konsul ('. Ebenan. laselhist.

7189.1 b. Erw. Marambitsy. Wast-Madagaskar. Gesch. 1892 ron Wr. Alfred Tocltzkow. Berlin.

7189,1 c. Halbw. Soalala. West-Madagaskat. Gesch. 1892 von demselben. 7189,1 1. + erw. Lajunga. West-Madagaskar. Gesch, 1892 rou demselhen. 7189.1 ¿. 2 ew. Kandani. S.-Bemhatokahai. Wost-Madagaskal. Gesel. 1892 von ilemselburl.

2. I) gomicodryas yuadrilineatus (D). B.).

Boulengar. ('at. I pag. 190): Boottger. Abl. Senck. Nat. Ges. Bil. 11. 1877 pag. 9, Bil. 11. 1879 pag 46:3 und Bel.12. 1881 pag. 4t3 (Herpetortryas hernieri var.).

$71 ! 0$ a. 5 elw.. 2 jung. Nossile. Gesch. 1876-83 ron Konsul C. Ebenau 111! Anton stumpfif. daselhst. 


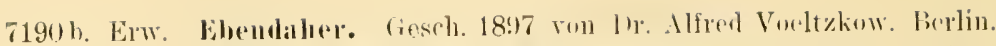

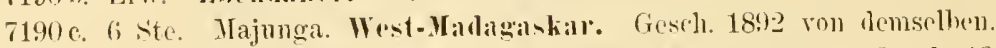

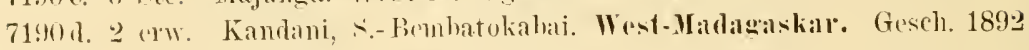
rom lemselhen.

71906. 2 erw. Soalala. Wext-Madagakkar. Gesels. 1892 rom demsellon.

\section{Genus III. Tinpidonotus Liull.}

1. Tropidonotus scalaris (cope).

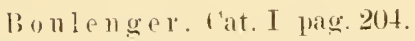

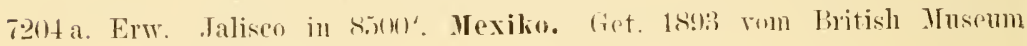
(ऽ. H.), Tomilom.

2. T'popidonotus "redinatus (L.). Bunlenger. ('at. 1 pag. 205.

ral. siltalis $\mathrm{L}$.

Bо и lеnger, 1. c. pag. 206 .

7206a. 4 Junge. Milwankes. Wisconsiu. Geselı. 189.) von Bierhrauer Rudolf Henrich. licr.

7206 b. Erw. Baltimore. Get. 1873 rom Taturh. Musenm in Offenbach.

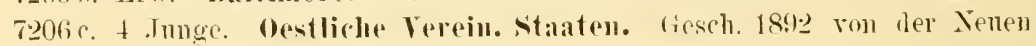
7oolog. Gesellscliaft, hier.

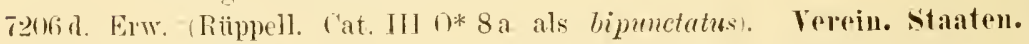
Gesell. 1840 ron Dr. merl. Remf nul W. Engrelnamu, st. Lonis.

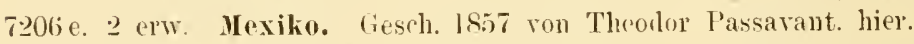

7206f. Erw. Florida. (resch. 1896 ron Dr. merl. A. Zipperlen, Cincinnati. ().

var. infernalis Blainr.

Boulenger, 1. c. pag. 207.

7207 a. Halbw, Californien. Crek, 1897 ron 1 A. Pöhl, Hamburg.

val. eques Reuß.

B о и lenger, l. с. pag. 209: Ren B. Mns. Senck. Bd. 1. 1834 pag. 152. Taf. 8, Fig. 2 (Coluber).

7209 a. 2 erw, (Riipnell, (at. III $0 * 81$ ) -c als bijunctatus). Mexiko. Gesch. 1832 von F. A. Lillenburger, Mexiko.

7209 b. Erw. (Rïppell. Cat. IIl O* $8 \mathrm{~d}$ als bipunctatus). Ehendaher.

7209 c. Erw. Mexiko. Gesch. 1896 von Dr. Heinrich Lenz, Lïbeck 


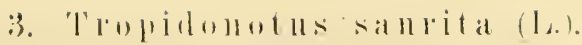

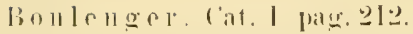

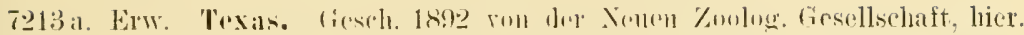

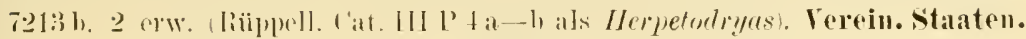

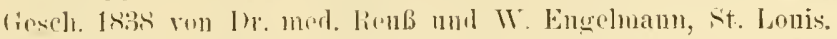

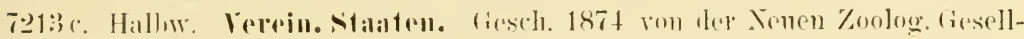
scllilft. liimer.

4. 'Tropidouotus pictulutus Schle.

lionlenger, ciat. I plage. 215.

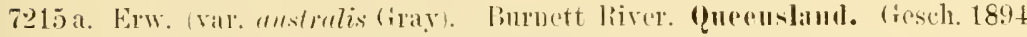
ron J'rof. I)r. Li. IV. Semon. Juma.

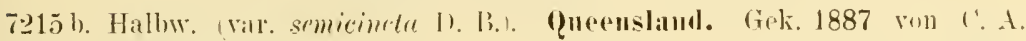
P'iille. Hamlunre.

5. 'Tropidonotus punctiventris Btgr'.

Bo paus. (it)2.

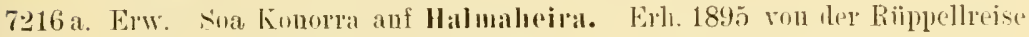
Prof. [lr. W. Kïilienthals, Jena.

6. Tropidouotus fuliginoilles (Gthr.).

Boulenger'. l'at. I pag. 217 .

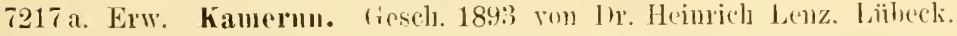

7217 b. Erw. Elhendaluer. (iescls. 1892 ron Prof. Mr. O. Boettger. hier.

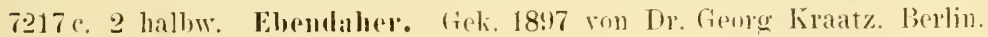

7. T'ropidonotus valiegatus (Pts.).

Bonlenger. l'at. I pag. 217.

7217,1 a. Erw. Ahuri, (iolllkiiste. Gesch, 1885 von Hans simom. Stuttgart

8. Tropidonotus natrix (L.).

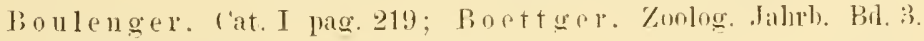
1888 pag. 933 .

7219 a. Erw. Dentschland. Geseh. 188.2 ron siegmund Wintex; hicx.

7219 b. Elw. Taumus. (iesch. 1889 ron Carl Happe. Lü̈lelleim.

7219 e. 2 erw. Kohyllno bei Oplyeln, Obersedelesirn. Gesch. 1880 von Otto Gollfub, Halls (siale).

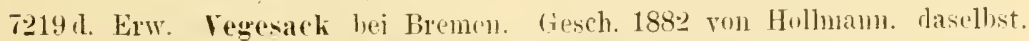

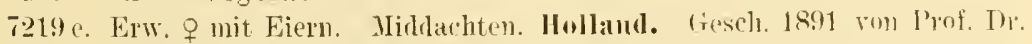
Fiedr. Kinkelin. lier. 


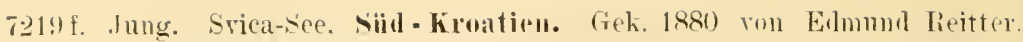
Paskau M[ähren!.

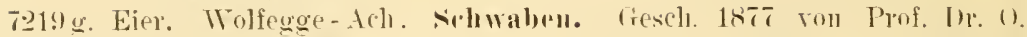
Boettger. lior.

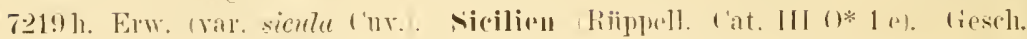

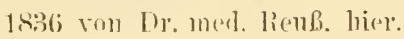

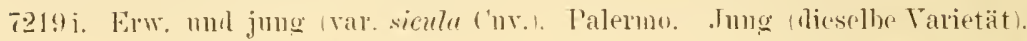
(Gltanisetta. Nirilien. Cisch. 1880 von ('hefinspektor carl Hirsch. daselhst.

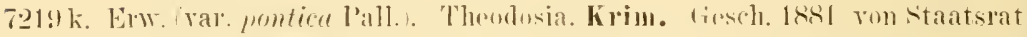
(). Letowski. dasollist.

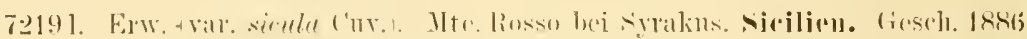
rom ('hefinspelitur latl Hirsch, I'alermo.

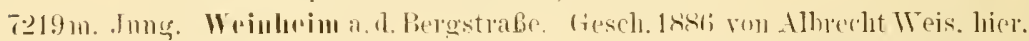

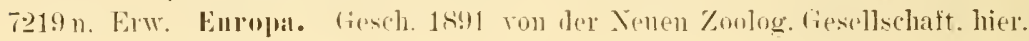

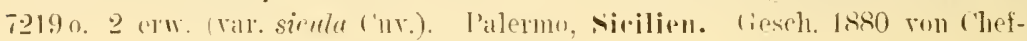

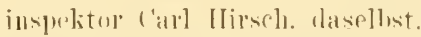

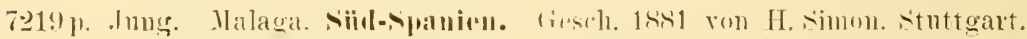

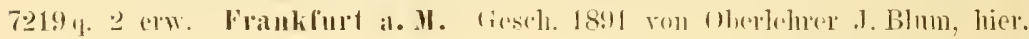

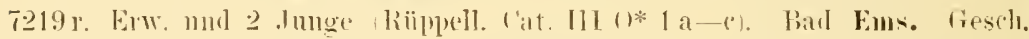

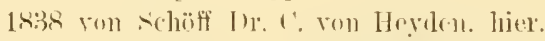

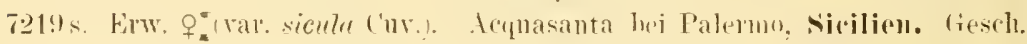
1886 rom ('hefinspektor ('arl Hirsch. l'alermo.

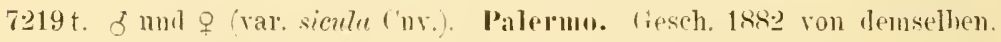

Val. pictulata Jan.

J a n. Ieon. "phid. lief. 206, Taf. 1. Fig. 1.

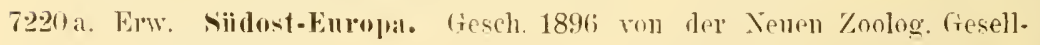
seliaft. lieer.

Var. persa Pallas.

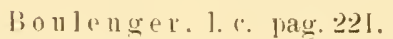

7221 a. Jung. Burgas. Ost-Rmurlirn. Gessh. 1894 rom 1hr. merl. ('. Flach. Aschaffenhurg.

72.21 b. Halbw. Kleinasien. Gesch. 18ti2 rom Aram Becker. Fydney.

7221 ๔. Hallw. Insel solta. Walmatien. (resch. 1891 rom Ir. Franz Werner; Wien.

7221 d. Elw. u. halbw. Prevesa. Epirns. Gesch. 1888 vou César coméménos, laselhst.

72201 e. Erw. Surma, Kleinasien. (iet. 1885 rom Naturh. Institnt Limnaea, Berlin.

7221 f. Kopf. Akerathal im Karahagh. Transkankasien. Erh. 1890 durch 1n. . Jean Talentins Reise.

7221 g. Erw. Karabagh. Transkankisien. Erh. 1890 von llerselben.

7221 l. Erw, see Beumbasch an der Atrekmündung, Transkaspien. Gesch. 1888 ron staatsrat Dr. (instar von Radile, Exe\%, Tiflis. 
7221 i. Erw. Siid-Europla. Gesch. 1893 ron der Nenen Zoolog. Gesellschaft, hier.

7221 k. Erw. Prevesa, Epirus. ('esch. 1889) von César Conéménos, daselhst. 72211. Erw. Patras. Yort-Morea. Gek. 18\%2 ron Dr. Christian Broemme, Wiesbaden.

$7221 \mathrm{~m} .2$ erw.. 2 jung. Chios. Gesch. 1862 von Hofrat Dr. med. Panli, hier. T221 n. 2 erw. Xenochori, Nord-Euböa. Giesch. 1891 ron Fr, de Mimont. daselbst.

7221 0. Jung. Trachori, Atolien. (iesch. 1892 ron Dr. ('hristian Broemne.

var. scut a t a Pallas.

Boulenger. 1. c. pag. 221 .

7221,1 a. Erw. Suidost-Europa. Gesch, 1896 von der Nenen Zoolog. Gesellschaft, hier.

i2.21,1 b. Erw. Katzensee, Kanton Ziurich. Schwri\%. Ciesch. 1897 ron Ur, merl Arthur Hanau, st. Gallen.

ral. atra Eichw.

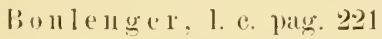

7221,2 a. Erw. Südost-Europa. Gesch. 189:3 von der Nenen Zoolog. Gesthschaft, hier.

7221.2 b. Erw, Riipuell. (at. 111 (1) 1 d). Italien. Get. 18t0 rom Hofmuseum in Wien.

9. 'Tropidonotus ribakari Boie.

boulenger. ('at. I pase. 20.21.

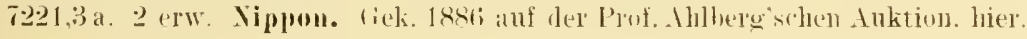

10. Tropidomotus conspicillatus Githr.

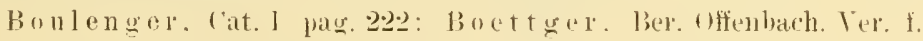

Naturk. 189.2 jage. 1019.

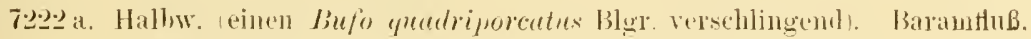

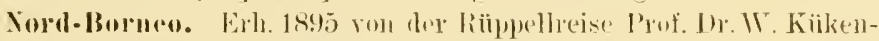
thats. Jena.

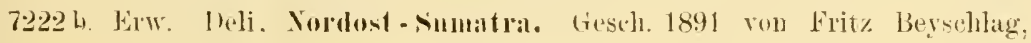
dacellst.

11. 'Tropidountus trianguligerus Boie.

boulenger. Cat. 1 page. 22-4.

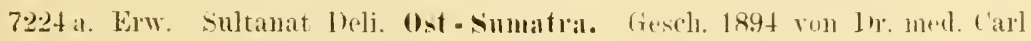
Flach, Aschatfenlumig: 
722+b. Erw. Baramfluß. Tord-Burneo. Erh. 1895 ron rer Riippellreise Prof. Dr. WT. Kükenthals. Jena.

722tc. Halbw. Insel Bangka. Gek. 1881 ron Konrad Kläsi. Zïrich.

7224 d. 9 mit Eiern ma 2 Junge. Buitenzorg. West.Java. Gesch. 1890 vou Dr. Adolf Strubell. Bomn.

\section{val. annulalis Fiscl.}

Bonlenger. 1. c. prag. 224 .

7224,1 a. Erw. Pontianak, West-Borneo. (iesch. 1896 ron Dr. Heinr. Lenz, Liibeck.

12. Tropidonotus olivaceus (Pts.).

Bonlenger, (at. I pag. 227; Boettger. Ber. Sencli. Nat. Lies. 1888 pag. 48 (cromella).

7227 a. Erw. Boma. Inter-('ongo. Cipl. 1886 ron Panl Hesse. Tenedig.

7227 b. Erw. Banana, Lnter-('ongo, Gek. 1887 von demselben.

7227c. Erw. Koliotoni anf Sansihaï. Gesch. 1897 ron Dr. Alfr. Vocltzkow, lierlin.

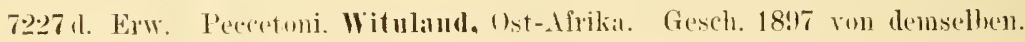

13. 'Tropidonotus piscator' (Sclund.).

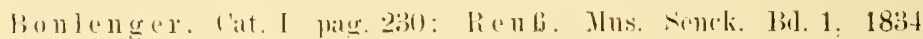

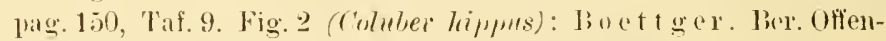
hach. Ver. f. Natmli. 1888 pag. 76 (quincunciutus).

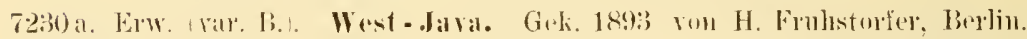

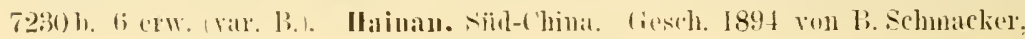
Fhanghai.

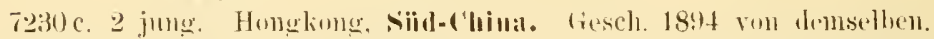

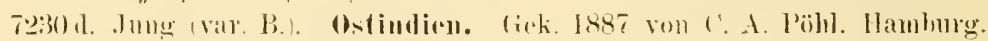

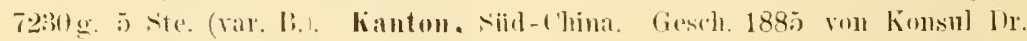
1). Fr. von Morelenderff. Manila.

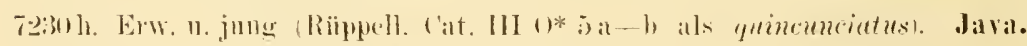
Gusch. $18: 37$ rom Thr. metl. P'eitsh. Batavial.

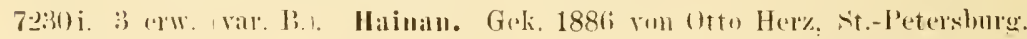

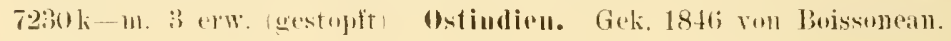

var. melanozosta frar.

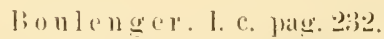

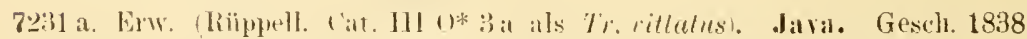
rent 1\%. II. rom siaboll. Leidem.

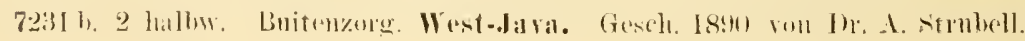
Bemln. 
14. Tropidonotus asperriuns Blgr.

lioulenger'. ('at. I pag. 232, Taf. 15. Fig. …

7230e. i) .Imuge. C'rylom. Gesch. 184fi ron H. Woums.

72301. Erw. Ehendaher. Gesch. 1881 ron Gustar Herath.

15. Tuopidonotus anumlaris Hallow.

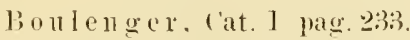

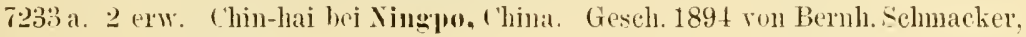
shanghai.

16. Tropidomotus tessellatus (Laur.).

Boulenger, Cat. I pag. 233: Boettger. ber. Renck. Nit. Ges. 1879-80 pag. 160, in Liadue's Famn. ('asp.-Gel). 1886 pag. 71 mol Zool. Jahth. Bit. 3. 1888 pag. 934.

7.234a. Erw. Schwehatthe hei Ballen nächst Wien. Gesch. 189:3 ron l)r. Fr. Werner. Wien.

7234 b. Erw. (var. flacescens Werm.). Zara. Dalmatien. Gesch. 1893 rom ¿lemselben.

723te. Jumg. Haiffi, Syrin. Gesch. 1879 ron Hans simon, Stuttgart.

7234 ג. 2 Junge. Jernsalem. Syrien. Gesch. 1880 rom ilemselben.

7234 e. Exw. 11. 4.Jnge. Brumána im Libanon. Gesch. 1881 ron denselben. 72:34t. Jung. Besch-tan bej l’jitigorsk. Ciskaukanien. Gesch. 1881 ron demselluen.

72:34g. Jung. I'etsch-deré. Abehasien. 'Tramskankasien. Erh. 1884 rom Statatsrat (Itto Jietowskis Reise.

7234h. Hallow. Nawaginskaja an P'schisch. Kulan scher Distrilit, (iskankasien. Gek. 1891 ron Panl Reihisch, Dresilen.

7234i. Jumg (Riippell. (at. IHI (1*20). Bal Ems. Gesch. 1838 von Schöff Dr. C. von Heyden. hier.

$7234 \mathrm{k}$. Elw. (Riippell. ('at. 111 ()*2f). Baslen bei Wien. Get. 1840) vom Hofmusenn, Wien.

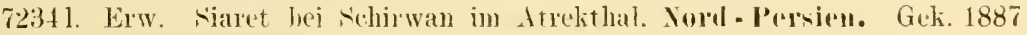
von (itto Her\%. st.-P'etershury.

$7234 \mathrm{~m}$. 2halhw. Askhahad. Transkaspien. Gesch, 1888-89 rom statsiat Dr. Gustar ron liadle, Excz. Tiflis, mul C. Eylandt, dasellost.

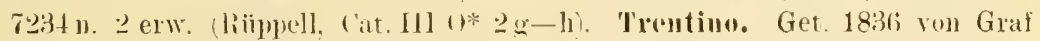
Irmison. Heilelherw.

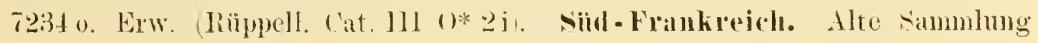
1845 .

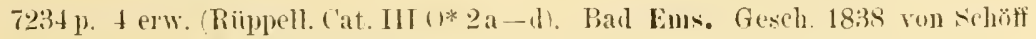
Dr. (¿. rom Heyden, hier. 


\section{Tropidonotus riperiuls (Latr.).}

Bonlenger. Cat. I jag. 235̃; Boettger: Abh. Senck. Nat. Ges. Br. 13. 1883 pag. 101.

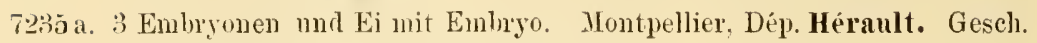
1887 ron .Joh. ron Fiselier, dlaselhst.

7235̆ b. Exw. n. 3 .Junge. lionte Tanger-Tetuan. Marokko. Gesch. 1881 ron Hans simon. sinttgart.

7235 c. Erw, Lantschaft Sus. Siid-Marokko. Gesch. 1872 ron Proff. Drr. ('. ron Fritsch. Halle (Saale), mil J. J. Rein, Bonn.

7235 ı. 2 erw., 2 jung. Algeciras. Sïl-spanien. Gesch. 1880 von Hans Simon. Stuttgart.

7235 e. Halbw, n. jung. Almuratiel. Sierra Morena. Sjanien. Gesch. 1868 ron Major In. L. von Heyclen. hier.

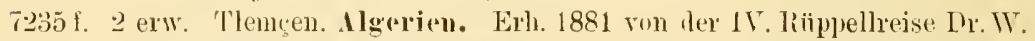
Kobelts. Sichwanhein a. M.

7235g. Jung. Lagos. Sïd-Portugal. Gesch. 1887 ron Prof. Dr. O. Boettger. hier.

T235 h. (i erw., 2 jung. Biscra. 11 werien. Gek. 1885 vou Lomis Fischer, dasellost. 7235i. Erw. Stalt Marokko. Gescls. 1872 ron Prof. Pr. (¿ rom Fritsch. Halle (Fiale).

\section{var. "cellata Wgl.}

B 011 lenger . 1. с. pag. 237 .

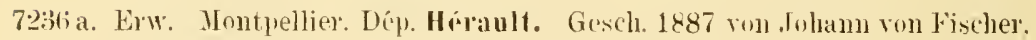
dasellist.

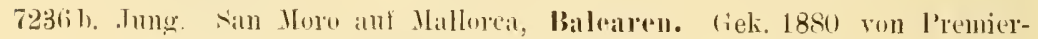
Lientenant I): Fr: Will, Erlangen.

7236c. Halhw. Algeciras, Niid-Npanien. Gesch. 1880 von H. Nimon. Stnttgart.

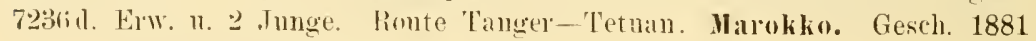
ron demselben.

\section{Tropidunotus ferox Githr.}

Bunlenger. ('at. J pratg. 2+1.

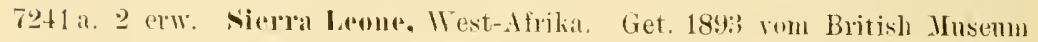
(X. H.). Lomdun.

19. T'น"uilunutus fasciatus I.).

Bunlenger: lat. I pag. 2t2.

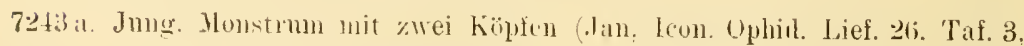

Fig. 2. san Antonin, Texas. Gesch. 1867 von P'rof. Dr. Rud. Chr. Boettger. hier:

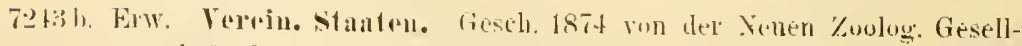
siclialt. hier. 
20. Tropidonotus dolichocercus (Peracea).

B oulenger, Cat. I pag. 246.

7246,1 a. Erw. đ. Moromanga, Ost-Madagaskar. Gescl. 1892 ron Dr. Alfr. Voeltzkow, Berlin.

\section{1. 'Tropidonotus stumpffi (Bttgr.).}

Boulenger, Cat. I pag. 247; B o ettger, Zoolog. Anzeiger 1881 pag. 358 und Abl. Scnck. Nat. Ges. Bd. 12, 1881 pag. 441, Taf. 1, Fig. 2 (Dromicus).

7247 a. 4 erw., 4 jung. Nossibé. Gesch. 1881-83 ron Konsul A. Stumpff, daselbst.

7247 b. Halbw. Ebendaher. Gesch. 1897 ron Dr. Alfred Voeltzkow; Berlin.

7247 c. Erw. Moromanga, Ost-Madagaskar. Gesch. 1892 ron demselben.

22. T'ropidonotus lateralis (D. B.).

Boulenger, Cat. l pag. 248.

7248 a. Erw. Najunga. West- Iadlagaskat. Gesch. 1892 ron Dr. Alfred Voeltzkow. Berlin.

23. T'ropidonotus platyceps Blyth.

Boulenger: Cat. 1 pag. 248.

7248.1 a. Erw. Darjeehng. Ost-Himiliyal. Get. 1891 rom British Museum (X. H., I.mollons.

24. Tropidountus tigrinus Boire.

Bonlenger. ('at. I pag. 244!.

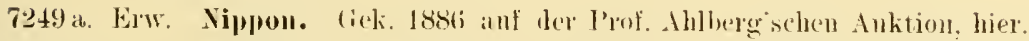

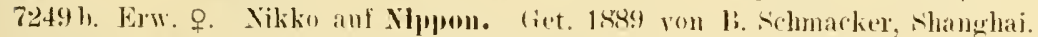

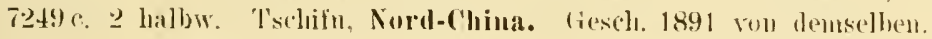

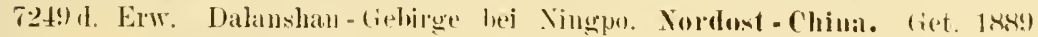
von demsellum.

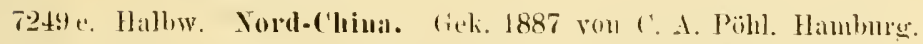

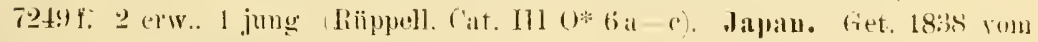
Rijkisinnsemm. Lejilen.

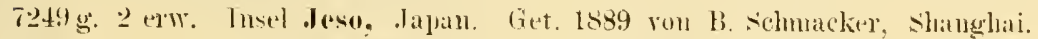

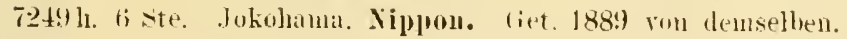

i249). Erw. Wei-hei-wri. Nord-China. (imeh. 1891 ron deusedluen.

7249 k. Erw, Liishan-Gehirge hej Kinkiang, Wst - China. Gret. 1889) ron dernselben.

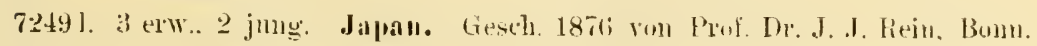

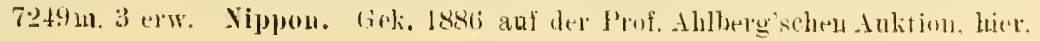


25. Tropidonotus pryeri Blgre.

Boule uger: Cat. I pag. 250.

7.5)a. Inng. Okinawa. Mittlere linkiu- Inseln. (iesch. 1895 von Bermb. schmacker. Shanghai.

7250 b. Erw, Yaerama anf Jijakoshima. Niidl. Linkin-Inselu. (iesclı. 189i) ron demselben.

26. Tropidonotus himalayanus Gthr.

boulenger. cat. 1 pag. 251 .

7251 a. Halbw. n. jumg. Darjeehng. Ost-Himalaya. Get. 1891 vom British Inusenm (.. H.). Londour.

27. 'Tropidonotus stolatus (L.).

bonlenger, ('at. I pag. 253: Boettger' Ber. Offenbach. Ver. f. Yaturk. 1888. pag. 79 .

7253 a. 7 erw. Hainan. Sïl-( hima. Gesch. 1894 ron b. schmacker. Slanghai. 7253b. Erw. Ceylon. Gek. 1887 ron ('. A. Pöhl, Hanburg.

7253c. 2 erw. Kanton. Siill-('hinal. Giesch. 188j yon Konsul Dr. 6. Fr. You Moellendorff, Manila.

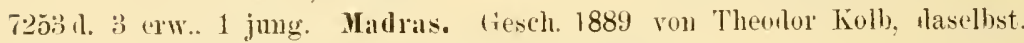
75̆3 e. Erw. Himalaya. tiek. 187 vou Hamburg.

$72003 \mathrm{ft} .2$ erw. Hainan. Gek. 1886 vou Otto Herz. St.-Petersburg.

7253 . Elw. Ceylon. Gesch, 1881 von Ginstav Herath. hier.

7253h. Erw. Yven-hong-li bei Hongkong. Siil-China, (iesch. 1880 ron Hans Simon, stuttgart.

72อ๊3 i. 3 erw. reylon. Gesch. 184t; ron H. Worms.

7.553. Erw. (gestopft). Ostindien. Gek. 1846 von Boissonean.

28. Tropidonotus vittatus (L.).

Boulenger. Cat. 1 pagr. $25 \%$.

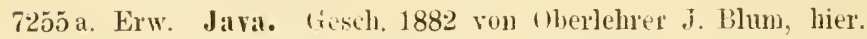

7255 b. 2 erw. Buitenzorg. West.Java. Gesch. 1890 von Dr. Arlolf strubell, Bomm.

7255c. Erw. Insel Bangka. Gek. 1881 ron Komral Kỉisi, Zürich.

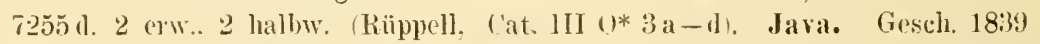
ron 1). merl. Peitsch, Batavia.

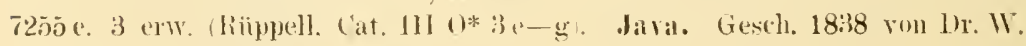
ron siebold. Lejilen.

29. Tropidanotus subminiatus Schlg.

Bonlenger. Cat. 1 pag. 25 i.

725 ia. Halbw. Java. (iesch. 1893 ron Albent 'Textor, Eisenach.

7256 b. Halbw. (Riippell. (at. I11 (1* 4 b als chrysorgus). Java. Geseld, 1838 rou Inr. med. Peitsch. Bataria. 
7256c. Halbw. Reich Jolo anf Jara. Gesch, 1868 ron E. Jörsch, hier.

7256 rl. Erw. Surabaya. Jara. Gesch. 1889 von Dr. merl. Stratz, Haag.

7256 e. Elw. (Riippell, Cat. III 0* 7 a als minatusi. Java. Fesch. 1840 von Dr. Wr. von sicbold. Leiden.

7256 t. 3 erw. Buiteuzorg, West-Jara. Gesch. 1890 ron Dr. Arlolf strubell. Bomm.

30. Tropidonotus spilogaster Boie.

Boulenger. Cat. I pag. $25 \%$.

7257 a. Erw. T'mgebung von Nanila auf Luzon. Gesch. 1891 von Konsul Dr. O. Fr. von IIoellendorff, Manila.

7257 h. 2 erw. Insel Celuí, Plilippinen. Gesch. 1890 von Otto Koch, laselbst. $72 j 7$ e. Zahlr. Ste. Thugebung von Manila, Luzou. Gesch. 1888 von Konsul Dr. O. Fr. von Moellendorff, Manila.

7:257 d. Ełw. Prov. Nneva Ecija, Luzon. Gesch. 1897 von demselben.

7257 e. Erw. Insel ('ulion, Calamianes. Gesch. 1897 ron demselben.

31. Tropidonotus chrysargus Schlg.

Boulenger. ('at. I palg. 258.

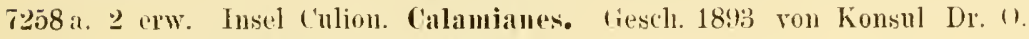
Fre von Mocllendorff, Manila.

7258b. Jnug. West-Java. Gek. 1893 von Hans Frulstorfer, Berlin.

7ะ5̆8c. Halbw. Stabat Estate bei Bindjey, Deli. Nordost-Sumatra. Gesch. 1893 von Fritz Beyschlag, daselbst.

720581. 2 Junge. Deli, Mordost-Simatra. Gesch. 187 !) von W. von Sehouler. Wiesbarlen.

7258c. Enw. Tengger-Gelirge in 1200 m, Ost-Java. Gek. 1891 roul Hans Frnlistorfer, Berlin.

$7258 \mathrm{f} .3$ halbw. 1 jung. Atschin, Nord-Sinuatra. Gek. 1882 von Komrat Kläsi, Ziirich.

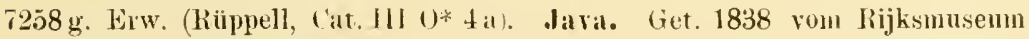
in Leidell.

7258 h. Jung. Banjemasin. Siidost-Borneo. Gek. 1894 von WWe. H. Krieh, hiel.

32. 'T'mpidonotus maculatus Erlel.

Bonlenger'. ('at. I pagig. $2(60$.

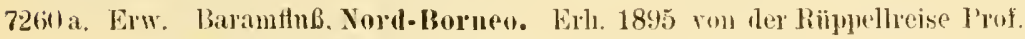

Dr. IV. Kïikenthals, Islia.

33. 'Tropidonotus chlysagoides CHur.

Boulenger, c'at. I pag. 2660, Taf. 16, lïg. 1.

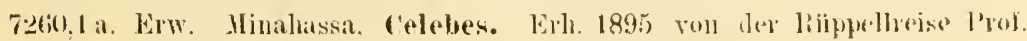

I). If. Küikenthals, fena. 
34. Tropidonotus auriculatus Gthr. B o u lenger, Cat. I pag. 261, Taf. 17. Fig. 1.

7261,1 a. Halbw. Insel Leyte, Philippinen. Gesch. 1893 ron Konsul Dr. O. Fr. von Moellendorff, Manila.

35. Tropidonotus callistus Gthr.

Boulenger, Cat. I pag. 263.

7263,1 a. Erw. Rurukan anf Celebes. Erlh 1895 von ter Prof. Dr. W. Kükenthal'schen Rüppellieise.

36. Tropidouotus halmaliericus Bttgr.

Boulenger, Cat. III pag. 609; Boettger. Zool. Anzeiger 1895 pag. 130.

7264.1 a. Erw. u. jung. Insel Bat jan. Erlh. 189.) vou der Rüppellreise Prof. Dr. IV. Külkenthals, Jena.

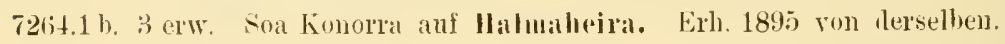

Genus II. Styporlynnchus Pts.

1. Styuorlunchus tumeatus Pts.

Bonlenger. ('at. I pag. 216 (Tropitonetus).

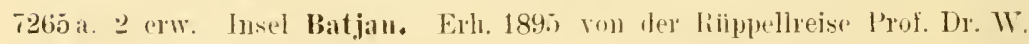
Kükenthals. Jena.

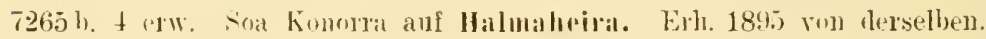

Grmes: $r$. Maciopisthodon Bilyr.

1. Ifacropisthodou flariceps (1). B.).

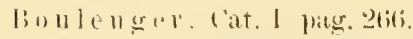

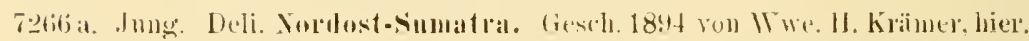

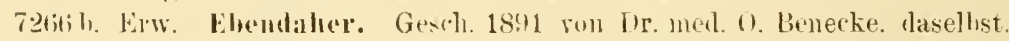
Feticic. Halhw. Ehendaher. Gesch. 1886 ron ('lemens Hohwiesner. hice.

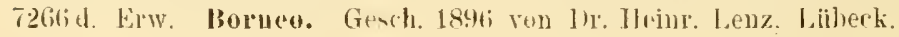

2. Macropisthadou rhodomelas (Boie). bonlenger. ('at. I prag. 266.

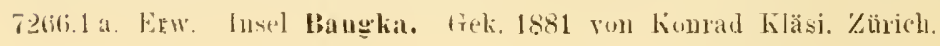


:. Macropisthodon plumbicolor (rant.).

Bonlenger. l'at. I page. otiz.

T2ti7 a. Erw. Korkaikanal. l'alni Hills. Niil-Indien. Fuseh. 1889 yon Throdor Koll. Marlras.

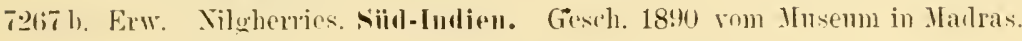

\section{Gems. T. Lioheterodon Blgr.}

1. Lioheterodon matagascariensis (1). B.).

Bonlenger. Cat, I pag. 269: Boettger. Abh. Senck. Sat. Ges. Bid. 11. 1877 pag. 5 mol Bel. 12. 1881 pac. 42 (Heterorlon).

7268a. 4 ite. Toxibe. Gesch. 1883 rom Konsul Anton stmmpft. daselbst. 7268b. 2 rew. Ebendaher. (iesch. 1875 ron Konsul ('arl Ehenam. daselhst. 72ti8c. Frw. Majunga. West-Madagaskar. Gesch. 1892 rom Dr. Alfred Voeltzkow, Berlin.

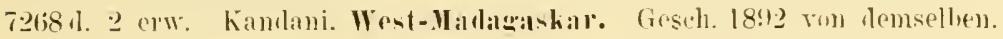

2. Iainhetelorlon morlestms (ithr.).

Boulenger. l'at. I pag. 269. Thif. 18. Fig. 1.

7269 a. 2 erw. Majunga. Wrst-Marlayaskal. fiesch. 1892 ron Dr. Alfred Voeltzkow, Berlin.

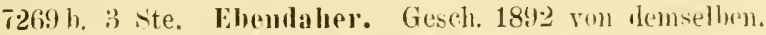

7269 c. Halbw, Soalala. Went-Matagaskar. Gesch. 1892 rou ilemselhen.

7อ69 d. 2 jung. Jajunga. West-Madagaskar. (tesch. 1897 ron demselhen.

\section{Cienus TII. I'seudorenorlon Blgi.}

1. Psendoxenodon macrops (Blyth).

Bonlenger, ('at. I pag. 27u.

7270a, Erw. Darje eling. Mst- Himalaya. Get. 1891 rom British Musemm (X. H.). Londom.

2. Psendoxenodon inornatns (Boie).

Bonlenger. Cat. I pag. 272.

7272a .Jung. Jara. Gesch, 1896 rou Direhtor Angust siehert, hier.

\section{Gemus TIII. Helicops Irgl.}

1. Helicojos sclistosus (Dand.).

Boulenger. Cat. I pag. $27 t$.

727ta. Jung. Kandy in Central-(Cylon. Gesch. 1890 vom Dr. Adolf strulell. Bomn.

727tb. Erw. C'rylon. Gek. 1889 von Hans Fruhstorfer. Berlin. 
2. Heliculs pictirentris Iremer.

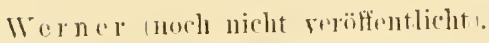

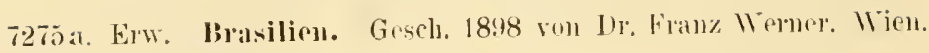

3. Heliculs callinicalla (Mied).

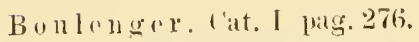

7276,1 a. Erw. Start Bio Grande. Rio diande do Sul, Brasilien. Gek. 1886 ron l'rof. Dr. H. von thering. daselhst.

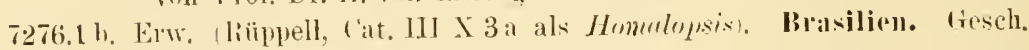
$1838 \mathrm{ron}$ sthoiff Dr. (․ ron Herden. hier.

Vial. inflataeniata Ian.

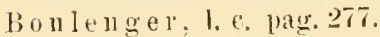

тет̆а. Erw. Rio drande do sul, Brasilien. Get. 1888 von Prof. Dr. H. ron Ihering. daselbst.

4. Helicops modestus Gthr.

Boulenger. Cat. I pag. $2 \overline{7}$.

7277.2 a. Erw, u. jung. São P’anlo. Brasilien, Gek. 1881 vom Jos. Duschanek. daselbst.

7277,2b. Halbw, n. jumg. Eheudaher. Gek. 187 ron Prof. cárlos Mïller. daselhst.

5. Helicops leopardinus (Schlg.).

Bonlenger, C'at. I pag. 278.

7278a. Erw. ('ujapa. Staat Matogrosso, Brasilien. Gesch, 1885 von Josef Schunacher, hier.

7278 b. Halbw. Mord-Brasilien. Gesch, 1897 ron Prof. Ir. (1. Boettger, hier.

6. Helicops augulatus (L.).

Boulenger, Cat. I pag. 278.

7278,1 a, 2 erw., 2 jung (Riippell, (at. III $x+a-d$ als Homalopsis). Ilheos.

Brasilien. Gesch. 1838 von Gebr. Koch, hier.

Genus IX. Tretanorhinus D. B.

1. Tretanolhinus nigroluteus Cope.

Boulenger, Cat. 1 pag. 282.

7282,1 a. Erw. Nicaragua. Gesch. 1892 ron l'rof, [1r. O. Buettger, hier. 
(ienlls: I. Isolmoymulhus: I). L'.

1. Is r hllonathus lekayi (Hollor.).

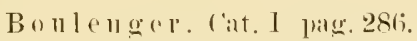

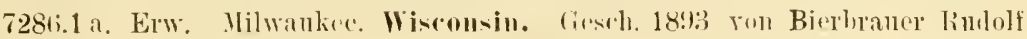
Henrich, hier.

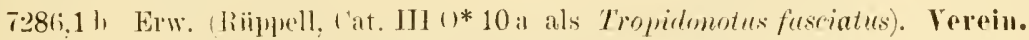

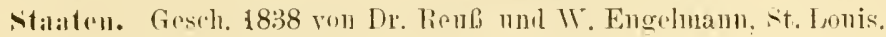

2. Ischnoguathus oceipitomatulatus (Stor.).

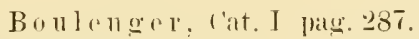

7.287 a. Halhw. и. 2 .Junge. Milwanke. Wisconsin. Geseh. 5893 rom Bierhraner limblf Henrich, hier.

3. Ischlloguathus storerioides (Cope).

B o 1 10 11 ger', l'at, I pag. 288.

7288 a. Erw. Jaliseo in 8500'. Hochland ron Mexikn. Get. 1893 rom British ILnsenm (X. H.). London.

(iemes XI. Streptophorus 1). B.

1. Streptophorus diadematus (B. G.).

Bonlenger. rat. I pag. 292.

7292a. Erw. Chiacany in 850 m, Guatemala. Gek. 1891 ron München.

2. Streptopliolus atratus (Hallow.).

B o ulenger, ('at. I pag. 293.

7293,1 a. Erw. Trinidad. Gesch. 1894 vom F. W. Trich mol R. R. Hole, Port of Spain.

$$
\text { var. lansbelogi D. B. }
$$

B o u lenger, l. c. pag. 294.

7294a. 2 Ste. Oropouche anf Trinidal. Gesch. 1895 ron denselben.

$$
\text { var. maculata Pts. }
$$

B о и lenger', l. c. pag. 294.

7294,1 a. 3 erw. San José, Costa Rica. Gesch. 1892-93 ron Carl Fleischmann. daselbst.

7294.1 b. Jung (schlecht gehalten). Ehendaher. Gesch. 1894 ron Konsul G. ron ichriter, daselbst. 
 \\ var. seliae 1). B.}

Bonlenger, ('at. I pay. 294.

7294,2a. 2 exw. Britixeh-Homduris. Get, 1893 rom British Musem (... H.). London.

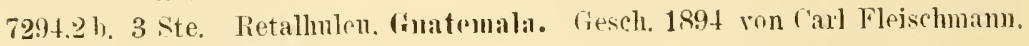
Quezaltenango.

Gemms XII. Trarthisctrimm Githi.

1. Trachischinm fuscum (Blyth).

Bo ulenger. ('at. I pas. 297.

7.297a. 2 erw. Darjeeling. Ost-Himalaya. (iet. 1891 rom Britisl Inseum (X. H.. London.

2. 'Trachischim guentheri Blgr.

Bonlenger. ('at. I pag. 298. Taf. 19, lïg. 1.

72:18 a. Erw. Ilarjeeling. Mnt-Himalaya. Get. 1891 rom Britixh Mnsemm (... H. . Lomilon.

\section{Gemes XIII. Oryrhabelimm Blgn.}

1. Oxyrhabdium modestum (D. B.).

Bonlenger. Cat. I pag. :302; Botetger. Ber. Senck. Nat, Ges. 1886 pag. 106 (partim).

7302 a. Halbw. Manila anf Luzon. Gesch. 1890 ron Konnsul Dr. O Fr. rou Iloellendorff, daselbst.

7302 b. Erw. ('entral-Ln\%on. Gesh. 1890 ron demselben.

7302 c. Elw. Insel Samal I'hilippincu. Geselr. 1897 ron demselben.

Genus III. Xylophis Bedrlome.

1. Xrlophis perroteti (D. B.).

Bonlenger. rat. I pag. 304.

7304a. Erw. Nilgherries, Niid-Indion. Gesch. 1890 som Mnsemm. Madras.

\section{Cenus. TI. Brachyorhus Boie.}

1. Brachyolous albus (L.).

Boulenger. l'at. I pag. 305.

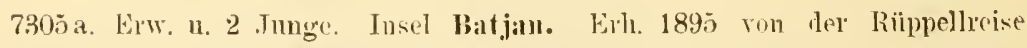
Prof. Dr. Wr. Kiikenthals. .Tena

73051). Erw. Insel Ternate. Erh. 1895 ron derselben.

7305̃c. 3 erw, n. 3 jung. Soa Konorra anf Halmaheira. Erh, 1895 rou dersethen. 


\section{Genus XVI. Elapoides Boie.}

1. Elapoides fuscus Boie.

Boulenger. Cat. I pag. 3017.

7:307 a. Erw. Buitenzorg. West-Java. Gesch. 1890 von Dr. Artolf strubell, Bolnn.

Genus XVII. Achatimus Its.

1. Achalinus spinalis Pts.

Bonlenger. ('at. T pag. 309.

7309.1 a. Erw. Hakonegehirge anf Nippou. Get. 1889 ron B. Schmacker. shanghai.

7:309,1 h. 2 erw. Japan. Giek. 188 6 auf der l'rof. Ahlherg'sehen Anktion. hier.

\section{Genns ITIII. Haplocereus Githr.}

1. Haplocercus ceylonensis Gthr.

Boulenger, ('at. I pag. 309.

7:310a. 4 erw. (Riippell, Cat. Ill ( 6 a - d als Culamaria seytrele). Ceylon. Gesch. 1846 von Wr. Worms. daselbst.

\section{Genus TIX. Aspidura Wyl.}

1. Aspirlura tracliyprocta Cope.

Bonlenger, c'at. I pag. 313.

7313a. 22 erw. Ninwara Ellija, Ceylon, (iesch. 1890 ron Dr. Adolf Strubell. Bonn.

\section{Genus XX. Pararhadinaca nor.}

Oberkiefer zart und schlank, mit etwa 17 schwachen. nach hinten alluählich an Länge zunehmenden Zähnen in gleichen Abständen. der hinterste nur mïßig vergrößert. zusanmengedrückt; I'nterkiefer breit, kräftigr. mit 11 ziemlich stumpfen Zähnen. die vordersten klein. allın̈hlich nach hinten größer werdend. vom sechsten an erheblich größer mul weitlänfiger gestellt. Kopf nicht vom Halse ahgesetzt: Auge klein. mit rmoler Pupille. Körpele walzenförmig. Schuppen glatt, olme Endgriilohen, in 17 Reilen: Ventral'n germotet. Schwanz kurz; Subcaudalen zweireihig. Hypapoplỵsen lïngs del ganzen Wirbelsäule deutlich entwickelt. - Nossibé.

Hierher als einzige Art:

1. Paralladinaea melanogaster n. sp.*)

7313.1 a. Erw. ․ Insel Yossibé. Gesch. 1897 von 1)r. Alfred Toeltzkow. Berlin.

*) Kopf niedergedliickt. Rostrale doppelt so broit wie lang. von when 


\section{Genus XXI. Lycognathophis Blgr.}

\section{Lycoguathophis sechellensis (Schlg.).}

Bonlenger, Cat, I pag. 317.

7317.1 a. Erw. Seychellen. Gesch. 1896 ron P'rivatdoc, I Zool. Dr. Ang. Braner, Jarkmie (Hessen).

eben noch sichtbar: Internasalsutur viel kïrzer als die Praefrontalsutur. Frontale zienlich dreieckig, vorn $1^{1 / 3}$ mal so breit wie lang und doppelt so breit wie ein Oculare. so lang oder etwas kürzer als sein Alstand von der Schnanzenspitze. viel kinrzer als the Parietalen. Oculare kleiner und viel schmiler als ein Praefrontale. Frenale fehlend: Yasale geteilt: das hintere Nasale stïßt an das T'raeoculare und trenut das l'raefrontale von den Supralabialen: zwo Postocularen. Temporalen $1+2$. las vorlere nicht mit dem oberen Postoculare in Berülnumg. Sieben supralahialen. ron denen das rlritte nurl vierte ans Ange treten. Tier Infralabialen in Berïhnung mit den vorderen Kinnschildern. die so lang sind wie die hinterm. Das vierte lnfralabjale sehr grob: das lintere submentalenpar billet nur mit je einem Infralahiale Sutur.

$$
\text { Schuppenformel: Sign. 17; G. }{ }^{5} 4 \text {. T. 163. A. }{ }^{1 / 1} \text {. Se. }{ }^{41 / 41}+1 \text {. }
$$

Hell luranngelb mit fünf scharf markierten dunkelbraunen Längsstreifen, von denen die änßersten am breitesten sind. dam folgt der Mittelstreifen; am sehmalsten sind die dazwischen liegenden Inssolateralstreifen. Die jerlerseitigen heiden Seitenstreifen vereinigen sich an der sichwanzwnzel zu einem eiuzigen Streifen. so dab der Schwanz nur drei dunkle Streifen zeigt. Vorn reichen diese Streifen his an ein weiBgelbes breites Band, das yuer über das Hinterhanpt zieht: nur die än fort und ziehen durcls Ange his zum Nasenloch. Die Kepfoherseite trägt eine dunkelluranne, weiß unsäunte Kappe. Ilie oberlippe ist zum größten Teil hell gefïrht. Kehle nnd Kim siml milchweiß mit je einem schwarzen Flecken anf jeder schuppe, die Ventralen schwarz mit weißem Seitenrand; anf der nntersten milchweiß gefärbten schuppemreihe eine fortlaufende Reihe feiner schwarzer Pünktchen. Subcaudalen milchweiß, in der ersten Schwanzlı̈̈lfte jedes Sulıcandalschildehen mit einem giroBen schwarzen Querflecken.

Il a be: Totallänge des erwachsenen $q$ : 239 . Schwanzlänge $37 \mathrm{~mm}$.

Fundort: Yossibé, sehr selten. nur in einem stïck, einem eiertrïchtigen of ron Herrn Inr. Alfred Voeltzkow aus Berlin gesammelt mul eingeschickt. Ein zweites, aber lileineres Exemplar befindet sich in der sammlung des Naturhistorischen Mnseums zn Lübeck.

Pe enerknngen: Diese kleine Art erimnert in Gestalt und Färbung sehr an die Gattung Elapotimes Ian. ist aber aglyphodont, hat zahlreichere Zähne in Oberkiefer nnd nach hinten größere mu weitläufiger gestellte ziilıne im Unterkiefer. 
Gems XXII. Ablabophis Blgr.

1. Ablabophis rufulus (Licht.).

Boulenger. I'at. I pag. 318.

7318 a. Erw. Siill-Afrika. Tiesch. 1873 von l'r. Lïwenthal, hier.

7318 h. Erw Eloendalere. (Riippell, Cat. III (f $t$ a als Coronella rufeserns.) Gesch. $18: 38$ von Baron von Lndwig.

\section{Ciemus XYIII. Tetralepis Bttgr.}

1. 'T'etralepis fruhstorferi Bttgr.

Bonlenger, ('at. I pag. 320): Boettger, Ber. uffenbach. Ver. f. Naturk. 1892 pag. 125.

7320 a. 2 erw. Tengger-(Gelirge in $1200 \mathrm{~m}$. ()st-Java. Gek. 1891 von Hans Fruhstorfer, Berlin.

Gemes XYIT. Lamprophis Smith.

1. Lamprophis a uropa (L.).

Boulenger, (at. I pag. 321.

7321 a. Erw. (Riippell. (at, III is 8 a als Cormella). Siid-Ifrika. Alte Sammlung 1845 .

\section{Genus XYI. Gonionotophis Blgr.}

1. Gonionotophis vossi (Bttgr.).

Bonlenger, ('at. I pag. 323; Boettger, 7ool. Anzeiger 1892 pag. 318 (Gonionotus).

7323,1 a. Halbw. Kamerum. Gek. 1897 ron Lr. Georg Kraatz. Berlin.

Genus IXYI. Cyclocoms D. B.

1. Cyclocorus lineatus (Reinh.).

Bonlenger, Cat. I pag. 327.

7327 a. 3 erw. Central-Luzon. Gesch. 1890 von Konsul Ir. O. Fr. von Inollendorff, Manila.

7327 b Halbw. Insel Samar, Philippinen. Gesch, 1897 ron demselben.

\section{Gemus IXITI. Boodon. D. B.}

1. Boodon geometricus (Schlg.).

Boulenger, ('at. I pag. 329.

7329 a. Erw. Seychellen. Gesch. 1896 ron Dr. Ang. Braner, Marburg (Hessen). 


\section{Boodon lineatus D. B.}

Boulenger, Cat. I pag. 332: Boettger, 24./25. Ber. Offenbach. Ter. f. Naturk. $188 j$ pag. 184 unt Ber. Senck. Nat. Ges. 1887-88 pag. 69 und 1889 pag. 294.

7333 a. 6 Ste. Banana. Unter-Congo. Gek. 1885 ron Panl Hesse, Venedig. 7333 b. Hablw. Odumase. Ost-froldkiiste. Gesch. 1892 ron Hans Simon, Stuttgart.

73333 c. Tung ( var. cariegute .Jan), Nïd-Afrikil. Gek. 1887 von C. A. Pöhl, Hamlurg.

73:33 ז. Erw. Kinshassa an stanley Ponl, Tuter-Congo. Gek. 1887 ron Paul Hesse. Tenedig.

73:33 e. Halhw. Akkra, Goldkiiste. Giesch. 1887 mn Major Dr. L. von Heyden, hier.

7333 f. Halbw. (var. quudrilineuta Jan). Port Natal. Gek. 1877 ron Hamburg. 73333 g. Erw. Insel S. Thomé. West-Afrika. (iesch. 1891 ron Dr. Jacques de Berlriaga. Nizzal.

7333h. Erw. u. jung. Kokotoni auf Sansiball. (iesch. 1897 ron [r. Alfred Toeltzkow, Beriin.

7333 i. Eıw. u. jung. Moschi bei T'anga, Kílinua - Njano. Fiesch. 1897 ron Ingeniem Karl Nolte. laselbst.

3. Boodon fuliginosus (Boie).

Boulenger, ('at. I pag. 334: Boettger, Ahl. Senck, Nat. Ges. Bet. 12, 1881 pag. 398 (unicolor).

7334 a. Erw. Akkra. Goldkiiste. Gesch. 188 r ron Iajor Dr. L. von Heyden, hier.

4. Boodon olivacells (A. Dum.).

Boulenger, Cat. I pag. 335.

7335,1 a. Halbw. Kanterun. Gesch. 1896 von Dr. Heinr. Lenz, Lübeck.

7335.1 b. Erw. Ebendaler. Gek. 1897 von Dr. Fenrg Kraatz. Berlin.

\section{Genus XXIIII. Lycophidium D. B.}

1. Lycophidium laterale Hallow.

B o u le ng er. ('at. I pag. 338; B o et tg er'. Ber. Senck. Nat. Ges. 1888 pag. 68 (capense var.).

73:38.1 a. Erw. n. halbw. Kamerum. (ick. 1897 von 1)r. Georg Kraatz, Berlin.

2. Lycophidinm capense (Smith).

Bonlenger, ('at. I pag. 339.

7339.1 a. Erw. (var. B), Kokotoni anf Sansihar. (resch. 1897 von Dr. Alfred Voeltzkow, Berlin. 
var. multimaculata Bttgr.

Bo o le uger, Cat. I pag. 340; B o ettger. Ber. Senck. Nat. Ges. 1888 pag. $6 \overline{7}$.

7340a. 2) erw. Banana, Cnter-Congo. Gek. 1887 ron Paul Hesse. Tenedig.

3. Lycophidium fasciatum (Gthr.).

Bonlenger, lat. I pag. 342, Taf. 22, Fig. 2.

7342.1 a. Erw. Kanterum. Gesch. 1892 von Prof. Dr. O. Boettger, hier.

7342,1 b. 2 erw. (schlecht gehalten). Ehendaher. Gek. 1897 von Dr. Georg Kraatz, Berlin.

Genus XYIX. Homonotus Hallou.

1. Holmoulotus modestus (D. B.).

Boulenger, Cat. I pag. $3+3$.

7344 a. Erw. u. jung. Odumase, Goldkiiste. Gesch. 1892 ron Hans simon. Stuttgart.

Gemus LXY. Lycodon Boie.

1. Lycodon striatus (Shaw).

Boulenger. ('at. I pag. 349.

7349 a. Erw. Ceylon. Gesch. 1881 ron Gustar Herath, hier.

7349 b. Iung. Madras. Gescli. 1890 ron Theodor Kolb, daselbst.

2. Lycodon aulicus (L.).

Boulenger. Cat. I pag. 352.

735̃a. Erw. (Rüppell, ('at. III L 1 a als hebe). Jara. Gesch. 1838 von Dr. ron Siebold, Leiden

7352 b. Erw. Bombay. Gek. 1877 von Hamburg.

7352 c. 2 crw. (Iiüppell, C'at. III V $11 \mathrm{a}$-b als Dipsas). Jara. Gesch. 1835 roll Dr med. Peitsch, Batavia.

$$
\text { var. capucina Boie. }
$$

Bonlenger; l. с. pag. 3533.

7353 a. Erw. Dapitan auf Mindana, (iesch. 1893 ron Konsul Ir. O. Fr. von Ioellendorff, Manila.

7353 b. Erw. Madras. Gesch, 1889 von Theodor Kolb, daselbst.

73533 c. 3 elw. Buitenzorg, West-Jara. Gesch. 1890 von Dr. Adolf Strubell. Bonn.

7353 d. 6 Ste. MitteI-Lızon. Gesch, 1890 ron Konsul Dr, O. Fr, ron Moellendorff, Manila.

7353 e. 22 erw. Manila anf Luzon. Gesch. 1891 von demselben.

7353 f. Jung. Insel Celui, Philippinen. Gesch. 1897 ron Otto Koch, daselbst. 
3. Lycodou travancoricus (Berld.).

Bonlenger. I'at. I pạ. 35\%, Taf. 24. Fig.3.

735.5 a. Halbw. Nilgherries, Niid-Indien. Gesch, 1890 vom Musenm in Madras.

4. Lycodon subcinctus Boie.

Boulenger, lat. I pag. 359.

7359 a. Jmug. Samarang. Jara. Gesch. 1876 ron Major Dr. L. von Heyden. hier.

7.35̆) b. Elw. 11. 2 halbw: Buitenzorg. West-Jara. (iesch. 1894) ron Dr. Adolf strubell. Bomm.

7359c. Halbw. Sultanat Deli, Nordust-sumatrid. Gesch. 1886 ron l'emens Hohwiesner, hier.

\section{Gemes IXYT. Dinodon D. B.}

1. Dinodon lufozonatus (C'ant.).

Bonlenger. (at. I pag. 361; Boettger. 24. 25. Ber. offenbach.

Ter. f. Yaturk. 1885 pag. 12t (var. formosenci und 1895 pag. 108.

7361 a. 3 erw. Yaeyama anf Jijakoshima, Nidd-Liukiu-(Aruppe. Gesch. 18!) ron Bernhard schmacker, Shanghai.

7361 b. Erw. (var. formostma Bttgr.). Insel Fornosit. (tesch. 1872 von Konsul Ferelinand Knoblanch. hier.

2. Dinoolon jayonicus (Gthr.).

Bonlenger'. ('at. I pag. 363 .

73633.1 a. Em. Nippon. Gek. 1896 von Gustar šchneider. Basel.

Gemus XYTII. Stegonotus D. B.

1. Stegonotus reticulatus Blgr.

Bonlenger. C'at. III pag. 619: Boettger' in Semon's Zool. Forsch. Heft 5. 1894 pag. 1:0 (modestus. non schlg.).

7366ia. Erw. Siidost-Teuguinea. (iesch. 1894 ron Prof. Dr. R. W. Semon. terna.

2. Stegonotus modestus (Schlg.).

Bonlenger. ('at. I pag. 36ti.

736tia. Erw. Yen-Gininea. Gesch. 1897 rom l'rof. Dr. O. Boettger. hier.

7366ib. Erw. Ema anf Leitimol. Niid-Inthoina. Gesch. 1890 roli Dr. Arolf Strubell, Bonn. 
3. Stegouotus heterurus Blgr.

Boulengel', Cat. I pag.367, 'Taf. 25, Fig. 1.

7367а. Halbw. Yeubritannien. Gek. 1887 ron C'. A. Pöhl, Hamburg.

\section{Stegouotus muelleri D. B.*)}

B o ulenger, Cat. I pag. 367.

7367.1 a. Halbw. Insel Samar, Philippinen. (iesch. 1897 ron Konsul Dr. O. Fr. ron Moellendorff. Manila.

5. Stegonotus batjaneusis (Gthr.).

Boulenger, Cat. I pag. 368 .

7368a. 6 ste. Soah Konorah anf Halmahera. Erh. 1895 von der Rïppellreise

Prof. Dr. Wr. Kiikenthals, Jena.

\section{Stegonotus dumerili Blgr.}

Boulenger, Cat. I pag. 368.

7369) a. Erw. Central-Luzon. Gesch. 1888 ron Konsul Dr. O. Fr. von Moellendorff, Manila.

\section{Genus IXXIII. Dryocalamus Gthr.}

1. Dryocalamus nympha (Daud.).

B oulenger, C'at. I pag. :370.

7:370a. Jung. Madras. Gesch. 1889 ron Theodor Kolb, daselbst.

7370b. Erw. (Riippell, Cat. III II 2 a als Clelia sp.). Ceylou. Alte Sammlung $18+5$.

*) Ahweichend von der (originalbeschreibung rurch 8 (nicht 9) Supralabialen. von denen das rierte und fünfte ans Auge treten.

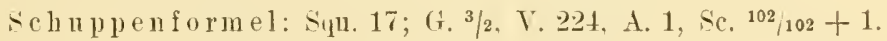

$$
\text { , } 17 ; 3 / 2, \quad 232,1, \quad{ }^{78} / 78+\text { ? }
$$

Rumpf (bei halbwïchsigen Stiicken) mit etwa 17-20 breiten schwarzen Querbinden. die durch schmale, anf der Rückenmitte nur etwa zwei Schnppenreihen breite, nach den Tentralen hin verbreiterte weiße Querbinden ron einander getrennt werden. Diese weißen Querbinden zeigen bräunliche Punktflecken. Lippen und L'nterseite weiBgelb, Bauch nach hinten häufig mit hreiten grauen Quergiirteln, die gegen den After hin immer breiter und dunkler werden und sich auf der Schwanzunterseite stets zu einfarbig schwarzgraner Färbung verdichten. 
2. I) ryocalamus darisoni (Blanf.).

Bunlenger, ('at I pag. 372; Buettger. Bir. offenbach. Ver. f. Niturk. 18 s s pag. 55 (T'lupe).

7372.2 a. Halluw. Bangkok, Nian. Gesch. 1893 ron Dir. Dr. Erich Haase. lasclbst.

7372 b. Erw. Eheudaher. Giek. 1886 von ()tto Herz, St.-Petershmg.

\section{Genus TIYIT. Psendaspis Cope.}

1. Pseudaspis cana (L.).

Bonlenger, Cat. I pag. 373.

7373a. Haibw. Damaralant. Gesch. 1893 ron Dr. Eil. Fleck. Azmga (Rumiinien).

7373 1). Halbw. (liiippell. (at. 111 o I2 a als Coluher). Kapland. Alte Sammlung $18+5$.

Var. Jhocartum Gthr.

Bonlenger. I. c. pag. $37 t$.

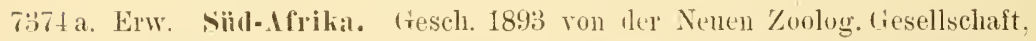
licer.

\section{Gemes IIIT. Zaorys Cope.}

1. Zarocys dhumbades (rantor).

Boulenger. l'at. I pag. 375. Taf. 26. Fig. 1.

7375 a. Erw. n. jung. Shinghai. Get. 1889 ron Bernh. schmacker, laselbst. 7375l. Enw. Lüshan-diebirge be Kinhiang. Get. 1889 ron demselben.

7375 с. Erw. Hongliong. Niid-China. Gek. 187 von Hanburg.

2. Laveys nigromarginatus (Blyth).

Boulenger, Cat. 1 pag. 376.

737tia. Halbw. Darjeeling, Mst-Himalaya. liet. 1891 rom British Musemm (X. H.). Lomilon.

3. Zaocys luzunensis Gthr.

Boulenger', ('at. I pag 37t, Taf. 26, Fig. 2 .

73т rorff, Manila.

\section{Kaocys carinatus (Gthr.).}

Boulenger. Cat. I pag. 377, 'laf. 27, Fig. 1.

7377,1 a. Erw. $\delta$. Sultanat Deli, Nordost-Sumatra. Gesch. 1886 ron Clemens Hohwiesner, hier. 
5. Kaocys fuscus (Gthr.).

Boule uger. ('at. I pay. 378, Taf. 27, Fi⿺. 2.

7378a. Erw. Sultanat Deli, Nordost-Sumatra. Ciesch. 1886 rom Clemens Hohwiesner, lier:

Gemus XYITI. Zamenis Hgl.

1. Zamenis korros (Schlg.).

Bonlenger, Cat. I pag. 384 .

7384a. Erw. Nyen-hang-li bei Hongliong, Niid-Chinal. Gesch. 1880 von Hans Simon, stuttgart.

7354 b. Erw. Buitenzorg, West-Jara. Gesch. 1890 von Dr. Adolf Strubell, Bonn. 7384 c. Erw. Insel IIainan, Sür-('hina. Gek. 1886 von Otto Herz, St.Petershurg.

7384 d. .Jung. Samarang, Javil. Gesch. 1876 von Major Dr. L. von Heyden, hier. 7384 e. Erw. Kanton, Siid-China. Gesch. 1886 von Konsul Dr. O. Fr. ron Moellendorff, Manila, mil Otto Herz, st.-l'etersburg.

7384f. Erw. Reich .Tolo, Java. Gesch. 1868 ron E. Liirsch, hier.

\section{Zamenis mucosus (L.).}

Bonlenger. ('at. I pag. 385: Boettger, Zool. Jahrlu. Bd. 3. 1888 pag. 931 (Pty(ts).

7385a. Erw. Taiwanfoo anf Siid-Formosil. (resch. 1895 von Bermhard schmacker, Shanghiii.

7385b. 3 erw. Kanton. Siid-China. Gesch. 1885 ron Konsnl Dr. O. Fr. von Ioellendorff, Manila.

7:385 c. Erw. Insel Hainan, Sür-C'lina. Gek. 1886 ron otto Her'z. St.Petersburg.

7385 d. Elw. Hongkong, Niid-('hina. Gek. 1877 von Hamburg.

738ге. Erw. Ceylon. Gesch. 1846 vom W. Worms, lier.

7385 f. Erw. Hongkung. Get. 1887 von B. schmacker, Shanghai.

7385 g. 4 Junge. Ceylon. Gesch. 1846 ron W. Worms, hier.

7385 h mml i. 22 erw. (gestopft). Ostindien. Gek 1846 von Boissonean.

$$
\text { 3. Lamenis constrictor (L.). }
$$

Bonlenger: Cat. I pag. 387.

7387 a. - erw. (typ. n. var. flavirentris Say). Verein. Staten. Gesch. $187 t$ 11. 1891 ron der Nenen Zoolog. Gesellschaft, hier.

7397 b. 2 erw. (var. flacicentris Say). Verein. Staaten (Rüppell, Cat. III O כa als Coluber). Gesch. 1838 ron Dr. merl. Reuß und W. Engelmann, st. Lonis. 
4. Zamenis mentorarius (D. B.).

Boulenger, Cat. I pag, 389.

7389 a. Halbw. (Var. ohne Fleckenreihen anf den rorkeren Ventralen). Carácas, Veneznela.*) Gek. 1897 ron Ingenienr Miuller, hier.

\section{Zamenis flagellifolmis (Lam'.).}

Boulenger, Cat. I pag. 389.

7389, 1 a. Erw. Mexiko. Gek. 1846 ron H. Finck, Lumigsburg.

\section{Zamenis ta ell iatus (Hallow.).}

Bonlanger, C'at. I pag. 390 .

7390 a. Erw. (typ.). Mexiko. Gek. 185ㄹ von Lehrer Hollmann, Vegesack.

\section{Zamenis bitaeniatus n. sp.**)}

7392,1 a. Erw. Retalhulen, Gnatemala. Gesch. 1895 ron (arl Fleischman, Guatemala.

*) Sicherer Funtort! Vergl. auch Jan, Elenco sist. d. Otidi. Milano 1863 pag. 64 (Jus. Turin!. - Boulenger. 1. c., kennt lie Art nur aus Hexico und Guatemala mol zwar speciell von San Geronimo in der Vera P'az, rom Rio (lisey und ans Mexice.

**) Xäichstverwanlt $Z$. pulcherrimus (c'ope) ron Tehuantepec, aber nit etwas zahlreicheren Tentralen, vorn hreiterem mu iiberhaupt relativ liibzerem Frontale ma abreichender Fïrbung. - Maxillarzïhne 20, die beiden letzten nicht kröftiger und nur seln wenig länger als die rorhergehenden. Schnanze zicmlich stark vorgezogen. vorn alogestumpft. Tiostrale etwas hreiter als hoch. sein oben sichtlarer Teil nur etwa ein Drittel les Abstandes zwischen ihm nnd dem Vorderrand des. Frontale messund. Lnternasalen wenig kïrzer als die Iraefrontalen: Frontale vorn deutlich breiter als tas supraocnlare, nur etwa $1^{1 / 2}$ mal so lang wie vorne breit, etwas länger als sein Abstand rom Schnanzenende. kiirzer als die Parietalen. Frenale knapp $1^{1 / 2}$ mal so lang wie hoch: ein Pracoculare, las das Frontale nicht erreicht; Subocularen fehlen: zwei Pustocularen. das mutere kleiner; nur halh so hoch wie las obere; Temporalen klein. $2+2+2$. Yeun supralabialen. ron denen das vierte. fiunfte und sechate in den Angenkrtis treten; fünf Infralabialen in Beriilnung nit den vorderen Kimmschildern: hintere Kinnschilder länger als die voudern und von einander durch Schnppen getremnt. Schuppen glatt: Tentralen seitlich nur sehr undentlich stmmpf gewinkelt.

schuppenforme1: squ. 17: (i. ${ }^{1 / 1}$. T. 2. 2)8. A. ${ }^{1 / 1}$. Sc. ${ }^{142 / 142}+1$.

Oberseits bis zur (verrundeten) Bauchkante hell grünlichgrau mit jederseits einem vom Nasenloch durch das Auge an den Rückenseiten und dem schwanze entlang lanfenden, hreiten tiefschwarzen [orsolateralstreifen, der 
8. Zamenis gemonensis (Taur.).

Boulenger, C'at. I pag. 395; Boettger. Sitz.-Ber. Akad. Berliu 1888 pag. 183 und Ber. Senck. Nat. Ges. 1879-S0 pag. 151 (viridiflarus rars. asiuna et carbonariu).

7395a. Erw. Caltanisetta anf Sicilien. Gesch. 1880 von Chefinspektor ('. Hirsch, Palermo.

7395b. Erw. Olivuzza auf Sicilien. Gesch. 1880 ron demselben.

7395 c. 2 erw. (var. curbonuria Bonap.). Taormina anf Nicilien. Gesch. 1880 von demselben.

7395.l. Erw. (diesethe Varietiit). Palermo. Gesch. 1882 von demselben.

7395 c. Erw. 11alien. Gesch. 1876 ron Hofr. Prof. Dr. O. Bütschli. Heidelberg. 7395 f. Erw. Ebendaher. Gesch. 1891 ron Prof. Dr. med. Edinger, hier.

$7395 \mathrm{~g}$. 2 erw Patras, Nord-Morea. Gek. 1892 ron Dr. Christian Brömme, Wiesbadell.

7395 h. Erw. Italien. Gesch. 1891 ron Prof. Dr. merl. Edinger, hier.

7395 i. Halbw. Insel Capri. Gesch. 1891 von Oberlehrer .J. Blum, hier.

$7395 \mathrm{k}$. Erw. o (Rüppell, Cat. III O 2 a als Coluber viridiflacus). Triest. Get. 1838 von Michahelles, München.

73951. 2 erw. Palermo. Geseh. 1881 von Chefinspektor ('. Hirseh, laselbst. $7395 \mathrm{~m} .3$ jung. Caltanisetta auf Sicilien. Gesch. 1986 von demselben.

7395 n. Erw. (Riippell, Cat. III $02 \mathrm{~b}$ als Coluber viridifuens). Trentino. Get. $18: 38$ ron Graf Jenison. Heidelberg.

73950. 3 erw. (Rüppell, Cat. HI $020-1$ und 3 a als C. riridiflares und caspius). Knin, Dalmatien. Get. 1838 ron demselben.

7395 p. 6 erw. Palermo. Gesch. 1880 von Chefinspektor C. Hirsch, daselbst. 7395 i. 2 erw. (var. carbonaria Bonap.) Cap Gallo bei Palermo. Gesch. 1880 von demselben.

7395 r. Erw. n. jung. (Riippell. ('at. III $02 \mathrm{e}-\mathrm{f}$ als C. viridiflecus). SiidEnropa. Alte Sammlung 1845.

\section{var. trabalis Pallas.}

B ou lenger, l. c. pag. 396; Boettger , Ber. Scnck. Nat. Ges. 1889 pag. 206.

7396 a. Erw. Theodosia, Krim. Gesch. 1882 ron Staatsrat O. Retowski, dasellist.

7396ib. Erw. Sinope: Kleinasieu. Erh. 1888 ron Staatsrat O. Retowski's II. Reise.

7396 e. Erw. Insel Chios. Geseh. 1862 ron Hofr. Du. med. Pauli. hier.

drei schuppenreihen eimimmt, während der lurch liese Streifen begrenzte lelle Medianstreifen zwei Schmppenreihen breit ist. Kopf oben olivengriin; Lippen und die ganze T'nterseite einfarbig elfenbeinweiß.

II a Be: Totallänge 857. Scliwanzlïnge $302 \mathrm{~mm}$.

Fundort: Retalhulen in Guatemala. nur das vorliegende stick ron Herrn ('arl Fleischmamn 1895 gefunden und eingesandt. 
var. asialla Bttgl.

B о 1 lenger. 1. c. pag. 397.

7397a. 2 Ste. Jerusalem. Gesch. 1880 von Hans simon. Stuttgart.

7397 b. Erw. (melanutische furms. Ehendaher. Gesch. 1880 von demselben. 7397 с. Erw. (desgl.). Sarona bei Jaffa, Syrien. Gescb. 1881 ron demselhen. 7397 1. Jung. Syrien. Gesch. 1840 ron W. Kosenbach, hier.

7397 e. Kopf. Haiffa, Syrien. Gesch. 1880 von Hans simon, stuttgart. 7397 f. 3 halbw. Jerusalem. (iesch. 1881 ron demselben.

\section{Zalmentis dahli (Fitz.).}

Boulenger. ('at. I pag: 397: Boettger. Ber. Senck. Nat. Ges. $1879-80$ pag 153.

7397.1 a. 2 erw. (Rïppell. ('at. 1Il s 1 a als Tyrial. Dalmatien. Get. 1838 ron Michahe!les, Miinchen.

7397.1b. 4 Ste. Jerusalem. Gesch. 1881 ron Hans simon, stnttgart.

7397,1 c. Erw. Syrien. (iesch. 1840 yon W. Rosenbach. hier.

7397,1ґ. Kopf n. jung. l'revesa. Jirirns. liesch. 1890 von C'ésar Conéménos. dascellost.

7397,1 e. Erw. 1r. jung. Jaffa, Syriell. (iesch. 1879 ron Jrajor 11r. L. rom Heyten. hicr.

7397.1f. Erw. var. nojudum Eichw.) Oberer Araxes. Russisch-lmuenien. Erh. $18: 30$ ron Tr. Tean Talentin's lieise in den Karahagh.

10. Zamenis rloudorluachis Jall.

Bonlenger, Cat. I pag. 398: Buet tger . Zool. Anzeiger 18!3 pag. 118 Calucensis var.'subnigra!

7398a. Erw. Askhabad, Transkaspien. Gesch. 1889 ron L. Eylandt, dasclbst.

val. Iadacensis Anders.

Bо и le ngex, l. с. pag. :39!9.

7399 a. Erw. (liippell. ('at. III 1 If als Colnder flomlentus). Tor. Mrabien. Gesch. 1836 von Dr. Echaril Riirpell. hiser.

\section{Zamenis kalelini (Brandt).}

Boulengri. (at. I pag. 401: Boettger. Zuol. Jahrb. Bd. 3, 1888 pag. 928 (ventrimaculatis var.).

7t01 a. Erw. Askhabal, Transkaspien. Gesch 1890 ron ('. Eylandt, dasellsst. 7401 h. Erw. Durun. Transkaspien. Gesch. 1888 ron staatsrat Dr Gustal von Ratlle, Excz, Tiflis. 
12. Zamenis florulentus (Geoffr.).

Bonlenger, Cat. I pag. 402.

740:3 a. :3 halbw. (Rïppell, Cat. III O 1 d-e als Coluber. Nubien. Gesch. 1830 von Dr. Eduard Rüppell. hier.

7403 h. 3 erw. (Rïppell, (at. III 0 1 a-c a!s Coluber). Agypten. Gesch. 1836 von demselben.

7403 c. Erw. Ebendaher. Gesch. 1897 ron der Nenen Zoolog. Gesellschaft. hier.

7403 d. Jung (Rüppell. Cat. III O 15 a als Coluber floriceps). Ehendaluer. (reschr. 1836 ron Ir. Ednard Riippell, hier.*)

\section{Zamenis ravergieri (Ménétr.).}

Boulenger, Cat. I pag. 405: Boettger, Zool. Jahrb. Bu. 3. 1888 pag. 927 (var. fedtschenkoi).

7405, Erw. Oberer Araxes, Russiscli-lrmenien. Erh. 1890 ron Dr. Jean Valentin's Reise in den Karabagli.

7405 b. Erw. (var. fedtschenkioi Strauch). Askhahar. Transkaspien. Gesch. 1888 von Staatsrat Dr. Crustar von Radle. Excz., Tiflis.

14. Lamenis nummifer. (RenB).

Boulenger, lat I pag. 407: ReuB, Mlus Senck. Bd.1, 1834 pag. 135 (Coluber): Boettger, Ber. Senck. Nat. Ges. 1879-80 pag. $15+$ (rocergieri var.).

7407 a. Erw. (Original. Rüppell, Cat. III $04^{* a}$ als Coluber). Ïgypten. Gesch. 18:30 von Dr. Eduard Riippell, hier.

7t07 b. + halbw. (Rïppell, Gat. III $O f^{*}$ b-c). Syrien. Gesch. 1839 ron TV. Rosenbach, hier.

7407 c. $t$ halbw. Jerusalem. Gesch. 1881 ron Hans Simon, Stuttgart.

15. Zamenis algirus (Jan).

Boulenger, Cat. I pag. 408; Boettger in Kobelt's Reise Alg. Tunis $188 j$ pag. 459 .

$7+08,1$ a. 2 erw., 2 jung. Biscra. Prov. Constantine, Algerien. Giek. 1885 von Louis Fischer, daselbst.

7408,1 b. 2 erw. Ehendaher. Fek. 188i) von demselben.

*) Dieses noch sehr junge Stïck ist in ker Fürbung sehr eigentïmlich. liißt sich aber auf keine andere Art beziehen. Die schwarzen Querzeichnungen anf dem Kopfe sind sehr markiert, ler kumpf einfarkig hell. 
16. Zamenis hippocrepis (L.).

Boulenger. ('at. I pag. 409; Boettger, Abl. Senck. Nat. Ges. Bil.9, 1874 pag. 157, Bi. 12, 1881 pag. 374, Bd. 13, 1883 pag. 100

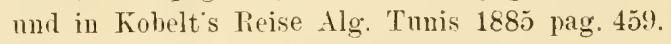

7ł09 a. Erw. o, jung u. Eier. Tunis. Gesch. 188 jo von Francesco Miceli. dasellist.

7409 b. Erw. Malaga. Sïd-Spanien. Gesch. 1881 ron Hans Simon. Stnttgart. 7409 c. Erw. n. 2 jung. Tlemçen, Ilgerien, Erh. 1881 von der IV. Rïppellreise Dr. merl. IV. Kobelt's, schwanheim (Main).

741)9 d. Erw. 11. 4 jung. Tanger, Inarokko. Erh. 1881 von derselben.

7t09 e. Erw. u. 2 jung. ('asablanca. Harokko. Gesch. 1881 ron Hans Simon, Stuttgart.

7409 f. Erw. Granada, Siiu-Spanien. Erh. 1881 von der IV. Rüppellreise Dr. med. W. Kobelt's.

$7409 \mathrm{~g}$. Jumg. Cartagena, Niid-Spanien. Gesch. 1881 von Hans Simon. Stuttgart.

7409 h. Kopf. Casablanca. Marokko. Gesch. 1889 ron Dr. merl. H. Schädle. raselbst.

$7409 \mathrm{i}$ nnd k. 2 erw. Lanlschaft Sus, Süd-Marokko. Gesch. 1872 ron den Proff. Drr. ('. v. Fritsch, Halle (Saale), und .T. J. Rein, Bonn.

7t091. Halbw. Stalt Marokko. Gesch. 1876 ron denselben.

7409 m. Halhw. Tuuis. Gescli. $188+$ von Francesco Miceli, daselbst.

\section{Zamenis diadema (Schlg.).}

Bonlenger. Cat. I pag. 411; B o ettger, Zool. Jahrb. Br. 3, 1888 pag. 92j und in Kobelt's Keise $\mathrm{Alg}$. Tunis $188 j$ pag. to 8 (rersicolor).

7411 a. 2 jung. Bisera, Prov. Constantine, Algerien. Gek. 1885 ron Louis Fischer, daselbst.

7111 b. Erw. Igypten. Gesch. 1886 von Prof. Dr. O. Boettger, hier.

$7+11$ c. 2 erw. (Rïppell, Cat. III $04 \mathrm{a}-\mathrm{b}$ als Coluber parallelus). Ehendaher. Gesch. 1834 von D1. med. Erluard Rïppell, hier.

7411 d. Erw. Sïd-Tunis. Gesch. 1896 von der Xenen Zoolog. Gesellschaft, hier.

Gemus XXXVII. Lytorliynchus Pts.

1. Lytorhynchus diadema (D. B.).

Boulenger, ('at. I pag. 415 : Boettger, Ber. Senck. Nat. Ges. 1878-79 pag. 61 (Simotes).

7415 a. 5 Ste. Jaffa, Syrien. Gesch, 1879-82 von Hans Simon, Stutgart, und Major 1r. L. von Heyden. hier.

$7+15$ b. Erw. Sïd-Tunis. Gesch. 1897 rom der Xenen Zorlog. Gesellschaft, hier. 


\section{Genus TXYVIII. Nenelaphis Gthr.}

1. Xenelaphis hexagonotus (Cant.).

Boulenger. ('at. snak. Brit. Musemu (N. H.) Bd. II. 1894 pag. 8. 8008 a. Erw. Stabat hei Binljey. Nordost-simmatra. Gesch. 1893 ron Fritz Beyschlag. daselbst.

8008 b. Erw. Bormen. Geseh. 1896 von Dr. Heinrich Lenz. Liibeck.

\section{Gemus XXXIX. Drymobius Cope.}

1. Drymobius bifossatus (Raddi).

Boulenger, Cat. Il pag. 10: Bo et tger, Zeitschr. f. d. ges. Jaturw. Bd. อ8. 188.) pag. 233 (Ptyos pantherinus).

Su10 a. Halbw. Varietït ohne jede dunkele Keichnung). Theos. Brasilien. (Riippell, ('at. III P 3 b) als Herpetodryas olfersi). Gesch. 1838 ron Gebr. Koch. hier.

8010b. Erw. São Paulo, Brasilien. Gek. 1876 von Prof. Cárlos Miiller. daselhst.

2. Drymobius boddaerti (Sentz.).

Boulenger, Cat. II pag. 11: Boettger. Ber. Senck. Nat. Ges. 1889 pag. 313 (Herpetodryas boddaerti rar. heathi Cope).

8011 a. 2 erw. (typ.). Insel Tohago, Westindien. Gesch. 1894 ron A. Seitz, Hamburg.

8011 b. 4 erw. (Rïppell, Cat. III P 7 b-e als Herpetodryas). Surinam. Gesch. 183i ron Dr. med. sichel. hier.

8011 c. Erw. (Rïippell, Cat. III P 7 a als Herpetodryas). Surimam. Gesch. 1836 von remselben.

8011 d. Erw. (rar. bitineata Jan). Brasilien. Gesch. 1886 ron Prof. Dr. Oskar Boettger, hier.

8011 c. Erw. (var. bitineata Jan). Trinidad. Gesch. 1893 ron F. W. Trich und R. R. Mole, daselbst.

8011 f. Erw. n. halbw. (typ.). ('arácas, Venezuela. Gek. 1897 ron Ingenieur Miiller'. hier.

$$
\text { var. heathi Cope. }
$$

Cope, Journ. Acad. T'hilatlelphia (2) Bul. 8.1876 pag. 179 (spec.).

8012 a. 3 elw.. 2 jung. Pacasmayo. Mord-Perí. Gesch. 1853 ron Max Bamberger, hier.

8012 b. Jung. Ehendaher. Gesch. 1897 fon demselben. 
var. rappi Gthr.

Boulenger. l. c. pag. 13.

8013 a. Halbw. Nordost-Brasilien. Gesch. 1886 von Prof. Dr. (). Boettger. hier.

8013 h. Jmng (Kiippell. ('at. IIl () 1ta als Coluber lichtensteini). Brasilien. Get. 1838 von Graf Jenison. Heidelberg.

8013 c. Jung. Trinidad. Gesch. 1879 von Carl Knoblanch. hier.

8013 ז. Inng. ('ancathal in $500 \mathrm{~m}$, I. S. Culumloia. Gek. 1897 von Prof. Dr. Fritz Regel, Jena.

8013 e. 5 .Tunge. Carícas, Venezucla. Gek. 1897 ron Ingenieur Miiller, hier.

Var. quinquelineata Strichr.

Boulenger. l. c. pag. 14 .

8014a. Erw. Alto Orennen. Niid-Veneynela. Gek. 1895 ron (Hentg Hiilner. Dresten.

3. Drymobius rlombifer (Gthr.).

Boulenger, Cat. II pag. 14.

8014.3 a. Halbw. C. S. Columbia. Gesch. 1894 ron Konsul F. C. Rehmann, Popayán.

4. Drymobius dendrophis (Schlg.).

Boulenger, Cat. II pag. 15.

8015̆,1 a. Erw. Puerto ('abello, Veneznela. Gesch. 1893 von Konsul F. Mauß. daselbst.

5. Drymobius margaritiferus (Schlg.).

Boulenger, Cat. II pag. 17.

8017 a. 2 Ste. Retalhulen, Gnatemala. Gesch. 189j von Carl Fleischmann, daselbst.

8017 b. Erw. Britisch-Fondnras. Get. 1893 vom Brit. Mnseum (N. H.), London. 8017 c. Ery. Mexiko. Gek. 1846 rom H. Finck, Lulwigshnrg.

Gemus XL. Phrynonax Cope.

1. Phryugnax sulphuleus (Magl.).

Boulenger, r'at. II pag. 19.

8019 a. Kopf u. Schwanz. Central-Trinidatl. Gesch. 1893 ron F. W. Trich und R. R. Mole, Port of spain. 
2. Phryouax poecilonotus (Gthr.).

Boulenger, Cat. II pag. 20.

8020 a. Erw. U. S. Colmmbia. Gek. 1896 ron W. F. H. Rosenherg, London.

3. Phrynonax fasciatus (Pts.).

Bonlenger. Cat. II pag. 21.

8021,1 a. Jung. (arenage bei Port of Spain, Trinidal. Gesch. 1895 ron F. IV. Irich mil R. R. Mole, daselbst.

4. Phrynonax entropis Blgr.

Boulenger, Cat. II pag. 22, Taf. 1, Fig. 1; Boettger. Bull.

Trinid. Club. Bıl. 2. 1894 pag. 85.

802. a. Erw. C'aparo auf Trinidad. Gesch 1894 ron F. W. Urich und R. R. Mole. Port of Spain.

\section{Genus XLI. Spilotes Tragl.}

1. Spilotes pullatus (L.).

Boulenger, ('at. II pag. 23.

8023 a. Erw. (var. B). Trinidad. Gesch. 1895 rou F. W. Trich und R. R. Iole, Port of Spain.

8023 b. Erw. n. jung. Insel Tobago, Westindien. Gesch. 1894 ron A. Seitz. Hamburg.

8023 c. Erw. (var. A). Trinidad. Gesch. 1893 ron F. W. Trich und R. R. Mole, Port of Spain.

8023 1. Erw. (var. A). Brasilien (Riippell, ('at. III 019 a als Coluber rariabilis). Get. $18: 38$ rom Naturh. Hofmuseum, Wien.

8023 e. Erw. (var. A ; gestopft). Brasilien (Rïppell, Cat. III (o 19 b als Coluber varialilis). Gesch. 1840 ron Frau ron Panhuijs. hier.

8023f. Erw. (var. A). Remerlios in 600 m. I. S. Columbia. Gek. $1897 \mathrm{rom}$ l'rof. Dr. Fritz Regel. Jena.

802:3 g. Erw. (var. A). ('arácas. Yeneznela. Gek. 1897 von Ing. Mïller, hier.

\section{Genus TLII. Colniver $L$.}

1. Coluber corais Boie.

Bonlenger', ('at. II pag. 31.

8031 a. Halbw. Trinidad. Gesch. 1893 ron F. W. Trich und R, R. Mole. Port of Spain.

8031 b. Jung. Ebendaher. Gesch. 1879 ron Karl Knoblanch, hier.

8031 c. Erw. Sorata. Bolivia. Gesch. 1894 ron Dr. Heimrich Lenz, Lübeck. 
var. melanura D. B.

B oul lenger, l. c. pag. 32.

8032 a. Halbw. (Riippell, Cat. III $\mathrm{G}+\mathrm{k}$ als Coronella cobella). Surinam. Alte Sammlung 1845 .

8032 b. Halbw. Gnatemala. Gesch. 1892 ron Prof. Dr. O. Boettger, hier. 8032 c. Erw. Mexiko. Gek. 1846 von H. Finck, Ludwigsburg.

2. Coluber porphyacens Cant.

Boulenger. C'at. II pag. 3 t.

8034 a. Erw. 11. halbw. Darjeeling, Ost-Himalaya. Get. 1891 rom British IIusenm (X. H.), London.

3. Coluber cantoris Blgr.

B oulenger, Cat. II pag. 35 .

8035 a. 2 halbw. Darjeeling. Ost-Himalaya. Get. 1891 rom British Museum (৯. H.), London.

4. Coluber leopardinus Bonap.

Bonlenger, Cat. II pag. 41.

8041 a. Halbw. Spalato, Dalmatien. Gek. 1880 von Edmund Reitter, Wien. 8041 b. Erw. Preresa, Epirus. Gesch. 1888 ron César Conéménos, daselbst. 8041 c. Erw. Jakirinitsa bei Volo. Thessalien. Gesch. 1888 ron Josef Stussiner, Laibach.

8041 d. 3 erw. (Ruippell, Cat. III O 7 a-c). Dalmatien. Fet. 1836 ron Dr. Michahelles. Miinchen.

8041 e. Erw. Sudak, Krim. Gesch. 1883 ron Staatsrat O. Retowski. Theodosia. 8041 f. Erw. Ehendaher. Gesch. 1884 ron demselben.

$80+1$ g. Erw. Prevesa, Epirus. Gesch. 1889 ron C'ésar Conéménos, daselbst.

$$
\text { var. quadrilineata Pall. }
$$

Boul enger, l. c. pag. 42.

8042 a. 2 erw. Insel Chios. Gesch. 1862 von Hofrat Dr. med. Pauli, hier. 8042 b. Kopf u. Vorderlälfte. Senochíri. Nord-Eubia. Gesch. 1891 ron Fr, de Mimont, daselbst.

\section{Coluber rufodorsatus (Cant.).}

Boulenger. ('at. II pag. 43; Boet $\mathrm{tger}$, Ber. Offenbach. Ver. f. Naturk. 1888 pag. 70 und Zool. Anzeiger 1886 pag. 519 (Simotes herzi).

8043 a. Erw. Hills bei Shanghai. Gek. 1886 von (1tto Herz, st.-Petersburg. 
80.43 b. Erw. Lüshan-Gebirge bei Kiukiang. Get. 1889 roı Perıh. Schmacker. shanglai.

8043 c. Erw. Sitai-See bei Slınghai. Get. 1889 von demselben.

8043 d. Erw. ('linthai bei Ningpo. Gesch. 1894 ron demselben.

6. Coluber dione Pallas.

Bon lenger. Cat. II pag. 44.

804ta. Erw. Tschiuas. Turkestan. Get. 1879) rom Naturh. Jusemm in St.Petersburg.

8044b. Erw. Tsehifm. Nort-(hina. Gesch. 1891 ron B. Schmacker. Shanghai.

\section{Coluber quatuorlineatus Lacép.}

Boulenger, ('at. II pag. 45).

8045 a. Kopf. Prevesa. Epirns. Gesch. 1890 mon f'ésar' Conéménos, llaselbst. 8045) b. Embryo. Dalmatien. Fesch. 1893 ron Schulrat Dr. Egid Schreiber. Gïrz.

8045c. Kopf u. jung. Xenochini. Nort- Eubial. Gesch. 1891 von Fr. de Mimont. daselbst.

8045 1. Erw. (Rïppell, ('at. III O 20)a). Dalmatien. Get. 1836 vou Dr. Michahelles, Mïnchen.

var. salu romates Pallas.

Bonlenger. l. c. pag. 47.

804 lia. Halbw. Smyna, Kleinasieu. Get. 1885 rom Naturh. Institut Limuea Ber'lir.

8. Coluber taeniurus (Cope).

Bomlenger, rat. II pag. 47; Boettger. Ber. Senck. Nat. ries 1894 pag. 144.

8047 a. Erw. Insel Formosa. Gesch. 1872 von Konsul Ferd. Knoblanch. hier. $80+7$ b. Erw. Shatughlai. Gek. 1886 von ()tto Herz. St.-Petersbmrg.

8047 c. Erw. Fluß W'usung hei Shamghai. Itot. 1889 von Bernhard schmacker. Shanghai.

8047 ๘. 22 erw. Shanghai. Get. 1889 von remselben.

8047 e. Erw. Siidcap ron Formosa. Giesch. 1891 ron demselben.

8047 f. 2 erw. Shanghai. Get. 1897 vom Naturh. Hofmusemm. Wien.

9. Coluber schrencki (Strauch).

Boulenger. Cat. II pag. 48.

8048a. Erw. Korea. Gesch. 1886 von Konsul Dr. O. Fr. von Moellendorff. Manila. 
10. Coluber obsoletus Say.

B oulenger, Cat. II pag. 5̃o.

8050 a. Erw. (gestopft). Vereiu. Staaten (Riuppell. Cat. III 0* 11 a als Tropidonotus poecilostoma). Gesch. 1840 ron Baron A. von Rothschild dinrch Prof. Fresreiß.

11. Coluber conspicillatus Boie.

Boulenger, Cat. II pag. 51.

8051 a. Erw. n. 2 halbw. Xippon. Gesch. 1876 ron Prof. Dr. J. J. Rein, Bonn.

12. Coluber longissimus (Laur.).

Boulenger. Cat. II pag. 52; B oettger. Ber. Senck, Nat. Ges. 1889 pag. 271 (aesculcupii).

8052 a. Erw. Trachori, Aetolien. Gek. 1892 ron Dr. phil. ('hristian Broemme, Wiesbaden.

8052 b. 3 erw. (Rïppell. Cat. II $06 a-c$ als flarcscens). SchIangenbad. Gesch. 1838 von Schöff Dr. Carl ron Herden. hier.

8052 c. 2 erw. (Rüppell, Cat. III $06 \mathrm{~d}-\mathrm{e}$ als flavescens). Trentino. Get. 1838 ron Graf Jenison. Heillelberg.

8052 d. Erw. Prevesa. Epirusus. Gesch. 1889 von César Conéménos, daselbst. 8052 e. Erw. IIte. Rossu hei Srrakns, Sicilien. Fiesch. 1886 ron Chefinspektor Carl Hirsch, Palermo.

8052 f. Erw. Caltanisetta auf Sicilien. Gesch. 1886 ron demselben.

8052 g. Erw. Schlangenbal. Gesch. 1888 ron Prof. Dr. Ferd. Richters, hier.

13. Coluber climacophorus Boie.

B oulenger, Cat. II pag. 54.

8054 a. Erw. Yippon. Gesch. 1876 ron Prof. Dr. J. J. Rein, Bonn.

8054 b. Erw. Hakone-Gehirge, Nippon. Get. 1889 ron Bernhard Schmacker, Shanghai.

8054 c. Erw. u. jung. Nikko, Nippon. Giet. 1889 ron demselben.

8054 d. 2 erw. Nippon. Gesch. 1876 yon Prof. Dr. J. J. Rein, Bomn.

8054 e. Erw. Jiyanoshita im Hakone-(iebirge. Nippon. Get. 1889 von Bernhard Sclmacker, Shanghai.

8054 f. 3 erw., 2 jung. Japrum. Gek. 1886 auf der Dr. Ahlberg schen Auktion. hier.

\section{Coluber moellendorffi (Bttgr.).}

Bonlenger, Cat. II pag. ó6; Boettger. Zoolog. Anzeiger 1886 pag. 520 und Ber. Offenlach. Ver. f. Naturk. 1888 pag. 72, Taf. 1. Fig. 1 (Cynophis).

8056,1 a. Erw. Nan-ning am Ju-tshang. Pror. ('unanghsi. Gek. 1886 von Otto Herz, St.-Petersburg. 
15. Coluber schmackeri Bttgr.

Boulenger, ('at. III pag. 627; Boettger, Ber. Offenbach. Ter. f. Naturk. 1895 pag. 108.

8056,2 a. Erw. Yaeyama auf Mijakoshima, Siidliche Liukin-Gruppe. Gesch. 1895 von Bernhard Schmacker, Shanghai.

16. Coluber oxycephalus Boie.

Bonlenger, Cat. II pag. 56.

8057 a. 2 erw. Buitenzorg, West-Jara. Gesch. 1890 von Dr. Adolf Strubell, Bonn.

8057 b. Erw. Malakka. Get. 1893 rom British Mlnseum (N. H.), London.

8057 c. Erw. Stabat Estate bei Bindjey, Sultanat Deli, Nordost-Sumatra. Gesch. 1893 ron Fritz Beyschlag, daselbst.

8057 d. Erw. Indrapura, West-Sumatra. Gesch. 1886 von Prof. Dr. Oskar Boettger, hier.

8057 e. Erw. Pontianak, West-Borneo. Gesch. 1896 von Dr. Heinrich Lenz, Liibeck.

17. Coluber quadrivirgatus Boie.

B oulenger, Cat. II pag. 59.

8059,1 a. 2 erw., 1 jung. Nippon. Gesch. 1876 von Prof. Dr. J. J. Rein, Bonn. 8059,1 b. Erw. Insel Jeso, Nord-Japan. Get. 1889 von Bernh. Schmacker, Shanghai.

805̃9,1 с. Erw. Nikko auf Nippon. Get. 1889 von demselben.

$$
\text { var. atra Jan. }
$$

B o u lenger, 1. c. pag. 60; J an. Jcon. Ophid. Lief. 24, Taf. 1, Fig. 3. $8059,-2$ a. Erw. Japan. Gek. 1877 von Hamburg.

18. Coluber melaumrus Schlg.

B o ulenger. C'at. II pag. 60.

8060 a. Erw. Karamfluß, Nord-Borneo. Erh. 1895 ron der Rüppellreise Prof Dr. W. Küikenthals, Jena.

8060 b. Halbw. Samarang auf Java. Gesch. 1876 von Major Dr. L. von Heyden, hier.

8060 c. Erw. Surabaya, Ost-Java. Gesch. 1889 von Dr. med. Stratz, Haag. 8060 d 3 jung. Buitenzorg, West-Jara. Gesch 1890 ron Lr. Adolf Strubell, Bonn.

8060 e. 2 erw. Sultanat Deli, Nordost-Sumatra. Gesch. 1894 von Wwe. H. Främer, hier.

8060 f. Erw. Atschin, Nord-Smmatra. Gek. 188ㄹ von Konrad Kläsi, Zürich. 8060 g. Erw. u. jung. Bindjey in Deli, Nordost-Sumatra. Gesch. 1890 von Gebr. Theodor und Egbert Engelhard, daselbst. 
8060h. Jung (Kïppell, ('at. IIL O 13a). Jata. (iet. 1840 rom Rijksmuseum, Leiden.

8060 i. 2 erw. Deli, Nordost-Sumat ra. Gesch. 1886 von Clemens Hohwiesner. hier.

19. Coluber radiatus Sclilg.

Bonlenger', Cat. II pag. 61.

8061 a. 2 jung. Buitenzorg, West-Jara. Gesch. 1890 ron Lr. Arulf strubell. Bonn.

$806[$ J). Jung (Rïppell. Cat. Ill 0 17 a). Javil. Gesch, 1833 rom Lr. Wr. ron siebold, Leider.

8061 c. Erw. Samarang anf Jiva. Gesch. $187(6$ von Mlajor 1)r. L. von Heyden, hier.

8061 d. Erw. Java. Giesch, 1886 von (H)erlehrer .1. Blmm. hier.

20. Coluber erythrulus (I). B.).

Boulenger, Cat. II pag. 6ะ.

8062 a. Erw. u. 2 jung. Manila auf Lazon. (iesch. 1891 von Konsul Dr. 6. Fr, von Moellendorti, daselbst.

8062 b. Erw. Tmgehung ron Manila. Luzon. Gesch. 1891 von demselben. 8062 e. 3 erw.. 3 jung. Nittel-Lızon. Gesch. 1888 ron demselben.

8062 d. Halbw. Insel Samar, ['hilippinen. Gesch. 1897 ron demselben.

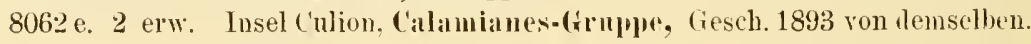

\section{Coluber scalaris Schinz.}

B о и le nger. ('at. II pay. 6.5.

subãa. Halbw. Spanien. Gesch. 1893 ron der Nenen Zoolog. Gesellschaft, hier.

8065 b. Halbw. Ebendaher. Gek. 1875 von Hamburg.

806 ã c. 2 halbw. Montpellier, Dép. Hérault. Gesch. 1887 ron Johann ron Fischer, daselbst.

22. Coluber depluei (I). B.).

Boulenger, Cat. II pag. 66 .

8066,1 a. Erw. (Rïppell, (at. III ()*9 a als Tropidonotus s1.). Mexiko. Gesch. 1842 rou F. A. Dillenburger, daselhst.

23. Coluber melanoleucus Daud.

Boulenger. Cat. II pag. 68.

8068 a. Halbw. Mexiko. Geseh. 1895 ron der Neuen Zoolog. Gesellschaft, hier. 8068 b. Erw. Ebendaher. Gesch. 1891 ron derselben. 


\section{Gemes XLIII. Herpetodryas $\mathrm{W}^{\top} g l$.}

1. Herpetodryas sexcariuatus (Wgl.).

B o n l e ge r, Cat. II pag. 72; B o ettger, Zeitschr.f.d.ges. Naturw. Bd. 58, 1885 pag. 233 (curinutus).

8072 a. 3) Ste. Sào P’aulo, Brasilien. Gek. 1876 und 1881 von Prof. Cárlos Miiller und Josef Duschanek, daselbst.

$$
\text { 2. Herpetodryas carinatus (L.). }
$$

B o ulenger, C'at. II pag. 73.

8074 a. 3 Ste. (var. macrophthalma Jan). Trinidad. Gesch. 1895 von F. IV. Urich nud R. R. Mole, Port of Spain,

807 tb. Erw. (Riippell. Cat. III P 1 a). Brasilien. Get. 1838 ron Graf Jenisou, Heidelberg.

8074 c. 2 erw.. 1 jung (Rïppell, Cat. III I' I d-e). Ebendalier. Alte Samm$\operatorname{lnng} 1845$.

807td. Erw. ㅇ u. 2 .Junge (Rïppell, Cat. III P $1 \mathrm{f}-\mathrm{g}$ ). Ilheos, Brasilien. Gesch. 1838 von Gebr. Koch, hier.

807t e. Erw. (var. mucrophthalmu Jan). Trinidad. Gesch. 1893 ron F. W. [rich und R R. Mole, Port of Spain.

8074 f. Erw. (typ.). Ebendaher. Gesch. 1893 von denselben.

$$
\text { var. bicarinata Wied. }
$$

B oul enger, 1. c. pag. 74 .

8074,2 a. Erw. San Iosé, Costa Rica. Gesch. 1894 ron Konsul Guido von Schröter, daselbst.

8074,2 b. Erw. (Riippell, Cat. III P 1 b). Brasilieu. Get. 1838 ron Graf Jenison, Heirlelberg.

8074,2 c. Elw. Rio trande do Sul, Brasilien. Gesch. 1873 ron Oberlehrer Dr. Finger, hier.

8074,2 d. Erw. ․ San José, Costa Rica. Gesch. 1892 von Carl Fleischmann, Guatemala.

8. 74,2 e Erw. ठ̛ и. భ. São Paulo, Brasilien. Gesch. 1890 von Prof. Dr. Osk. Boettger, hier.

8074,2 f. Erw. Brasilien. Gek. 1887 ron C. A. l'öhl, Hamburg.

8074,2 g. Erw. Rio (xraude do Sul. Gek. 1887 von Prof. Dr. H. von Ihering, daselhst

$807 t, 2$ h. Erw. Surata. Bolivia. Gesch. 1891 von Dr. Heinrich Lenz, Lïbeck. 8074,2 i. Jung (Rïppell, Cat. III T 4 a als Dendrophis laevicollis). Brasilien. Alte Sammlung 1845.

$$
\text { var. flavolineata Jan. }
$$

B o n l enger, C'at. II pag. 75; B o ettger. Zeitschr. f. d. ges. Naturw. Bd. 5x, 1885 pag. 234 (spec.).

8075 a. Erw. (Rüppell, Cat. III P 1 c). Brasilien. Get. 1838 von Graf Jenison, Heidelberg. 


\section{Genus XLIT. Dendrophis Boie.}

1. Dendrophis pictus (Gmel.).

Boulenger'. ('at. II pag. 78 .

8078 a. 2 erw. Baramtluß in Norl-Borneo. Erh. 1895 von der liiipellreise Prof. Dr. Wr. Kükenthals, Jena.

8078 b. Erw. Ema anf Leitimor, Niid-Amboina. Gesch. 1890 ron Dr. Arlolf Strubell, Bonu.

8078 c. Erw. Madras. (iesch. 1889 von Theodor kolh, daselbst.

8078 d. 4 Ste. (Riippell, ('at. III T $2 \mathrm{c}$-f). Java. (iesch. 1838 von Lr. von sieboli, Leilen.

8078e. Erw. Buitenzorg. West.Java. Gesch. 18!k von 1hr. Ailolf strubell. 8078f. Halbw. Balangan anf Borneo. Gesch. 1892 von I'rof. Dr. Oskar' Boettger, hier.

8078 g. 3 Ew. Bindjey, Sultanat Deli. Nordost-sumatra. Gesch. 1890 ron Theodor und Eghert Engelhard, daselbst.

80781. Erw. Deli. Nordont-Nimmatra. Geseh. 1894 ron Wwe. H. Krämer. hiel.

8078i. 2 erw. Imgebung ron Manila anf Luzon. Gesch. 1891 ron Konsul Dr. O. Fr. von Moellendorff, daselbst.

8078 k. Erw. Manila. Gesch. 1877 ron housul Ferd. Knoblancl. hier.

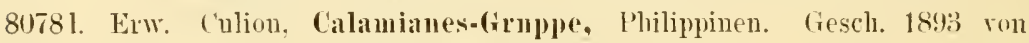
Konsul [2r. O. Fr. von Moellendorff.

8078 m. Erw. Surabaya. Ost-Jara. Gesch. 1889 vou Ihr. merl. Stratz. Haag. 8078 n. 6 erw. Atschin. Yorl-Sumatra. Gek. 1882 ron Komrarl Kläsi. Ziirich.

8078 o. 2 crw. (Rüppell, (at. III T 2 a-b). Jara. Get. 1839 rom Rijksmuseum in Leiden.

8078 p. Zahlr. Ste. Mittel-Luzon. Gesch. 1888 ron Kunsul Lr. O. Frr. ron Ioellendorff.

8078 . Erw. Banjermasin. Siidost-Borneo. (iek. 1894 vou Wwe. H. Krieh. lier.

2. Dendrophis calligaster Githr.

Boulenger, (at. II pagr. 80): Boettger in Semon's Zool. Forsch. Hft. 5. 1894 pag. 120 (punctulutus part.).

var. salomonis Gthr.

Bonle 11 ger, l. c. pag. 82.

8082,1 a. Erw. Siidost-Nenguinea. (iesch. 1894 ron Prof. Dr. R. W. Semon. Jena.

808:2,1 b. Erw. Insel Duke of Fork, Neubritannieu. Gek. 1887 ron C. A. Pöhl, Hamburg. 
3. Deudrophis punctulatus (Gray).

Boulenger. ('at. II pag. 82; Boettger in Semon's Zool. Forsch. Hft. 5. 1894 pag. 110.

8082a. Erw. Yord-Australien. Ciek, 1887 ron C.. A. Pöhl. Hamburg.

4. Dendrophis formosus Boie.

Boulenger. ('at. II pag. 84; 3oettger in semon's Zool. Forsch. HIft. 5. $189+$ plag. 12 ? (pictus).

8084,1 a. Erw. (Riippell. ('at. III T 3a). Java. Gesch. 1838 ron Ir, von sieboli, Leirlen.

5. Dendrophis lineolatus Hombr. Jacy.

Boulenger. (at. II pag. 85: Boettger in Semon's \%ool. Forsch. Hft. 5, 1894 pag. 120 (munctulutus part.).

8085,1 a. Erw. Palaı-Inselı. Gek. 1887 von C. A. Pöhl, Hamburg.

\section{Genus XLT. Dendrelaphis Blgr.}

1. Dendrelaphis tristis (Daudin).

Boulenger, Cat. II pag. 88.

8088 a. Erw. CeyIon. Gesch, 1881 von Gustar Herath, liee.

2. Dendrelaplis caudolineatus (Gray).

Boulenger, Cat. II pag. 89.

8089,1 a. Erw. Bindjey im Sultanat Deli. Nordost-Sumatra. Giesch. 1890 von Theodor und Egbert Engelhard. daselbst.

8089.1 b. 2 erw. Deli, Nordost-Smmatra. Gesch. 1892 von Dr. merl. (). Benecke, daselbst.

8089,1 c. Erw. Stabat Estate bei Bindjey, Deli. Nordost-Sumatra. Gesch. 1893 von Fritz Beyschlag. daselbst.

8089,1 d. Erw. Tontianak. West-Borneo. Gesch. 1896 von Dr. Heinr, Lenz. Liibeck.

3. Dendrelaplis terrificus (Pts.).

Boulenger. ('at. II pag. 90.

8090 a. Erw. ('rmoc auf Leyte, Philippinen. Gesch. 1890 von Otto Koch, Cebí. 8090 h. Halbw, Dapitan, Nord-Mindanao. Gesch. 1893 ron Konsul Dr. O.

Fr. von Moellendorff, Manila.

8090 c. 6 Ste. Mittel-Luzon. Gesch, 1890 von demselben. 
4. Dendrelaphis modestus Blgr.

13 onlenger, Cat. II pag. 91. Taf, 4. Fig. 4.

8091 a. 4 crw. Insel Batjan. Erh. 1895 ron der Rüppellreise Prof. Dr. W.

Kïlienthals. Jena.

8091 b. Erw. Insel Ternate. Erh. 1895 von derselben.

8091 c. 6 ste. Soah Konorah auf Halmahera. Erh. 1895 ron derselben.

\section{Gems YLIT. Chlorophis Hallou.}

1. Chlorophis hoplogaster (Gthr.).

Boulenger. Cat. II pag. 93, Taf.5, Fig. 2.

8093,1 a. Halbw. Boroma an sambesi. (resch. 1894 ron Prof. Dr. O. Boettger. hier.

2. C'hlorophis heterolepidotus (Gthr.).

Bonlenger, Cat. II pag. 95, Taf. 5. Fig. 3; Boettger, Ber. Senck.

Nat. Ges. 1888 pag. 60 (Philothammes).

8095,1 a. Erw. Boma, Cnter-Congo. (iek. 1887 ron Paul Hesse, Venedig.

3. Chlorophis irregularis (Leach).

Boulenger. Cat. II pag. 96; Boettger, Ber. Senck. Nat. Ges. 1888 pag. 61 (Philothammus).

8096 a. 2 erw. Poro Yetonna bei Banana, Unter-Congo. Gek. 1887 von Paul Hesse. Venerlig.

4. Chlorophis heterodermus Hallow.

Boulenger, Cat. Il pag. 97: Boettger, Ber. Senck. Nat. Ges. 1888 pag. 09 (Plitothemnus).

8097 a. Halbw. Povo Nemlao hei Banana. Unter-Congo. Gek. 1887 von Paul Hesse, Tenerlig.

8097 b. Erw. Kamernu. (iesch. 1893 ron Dr. Heinrich Lenz, Lübeck.

8097 c. Erw. Odumase.0st-(a)ldkiiste. Gesch. 1892 ron Hans Simon, stuttgart. 8097 ґ. Jung. Kanerun. Gek. 1897 ron Dr. Georg Kraatz, Berlin.

\section{Gemus ILVII. Phitothammes Smith.}

1. Philothamnus semivariegatus (Smith).

Boulenger, Cat. II pag. 99.

8099 a. 3 erw. Kokotoni auf Sansibar. Gesch. 1897 ron Dr. A. Voeltzkow, Berlin. 
2. Philothamuns dorsalis (Bocage).

Boulenger. Cat. II pag. 101; Boettgrr: Ber. Senck, Sat, Gies. 1888 pag. j8.

8101 a. I Ste. Banana, Unter-Congo. Gek. 1886 ron l'anl Hesse, Venedig. 8101 h. Erw. Elsendaher. Gek. 1887 von demselben.

3. Philothamuls thomensis Bucage. Boulenger, Cat. II pag. 101.

8101,1 a. Erw. Insel St. Thome, West-Afrika. Gesch. 1891 von Dr. J. von Berliaga. Nizza.

\section{Genus ILIIII. Gastropyxis Cope.}

Boulenger, c'at. 11 pag. 103; Boettger. Ber. Senck. Nat. Ges. 1888 pag. 62 und 1889 pag. 279 (Hapsidophlerys).

8183 a. Erw. Nierra Leone. Get. 1873 rom Katmih. Jlusem in (Iffenbach (Iain).

8103 b. Erw. Brass River, West-Afrika. Gesch. 1881) ron D. (1. W. Nabert, hier.

8103 c. Halbw. Cahinda, Cnter-('ongo. Ciek. 1885 ron Panl Hesse, Venedig. 8103 d. 2 erw. Banana, Unter-('ongo. Gek. 1887 ron demselben.

8103 e. Elw. Udumase, Ost-(Koldkiiste. Gesch. 1892 ron Hans Simon. Stuttgart. 8103 f. Halbw. Kamenun. Gek. 1897 von Dr. (teorg Kraatz, Berlin.

\section{Genus XLIX. Thrasops Hallou.}

1. 'Thrasops flavigularis (Hallow.).

Boulenger, ('at. II pag. 105; Buettger, Ber. Senck. Nat. Ges. 1889 pag. 279 (var. pustulutu).

8105 a. Erw. Povo Nemlao bei Banana. Vuter-('ongo. Gek. 1886 von Paul Hesse, Venelig.

s10ذb. 2 r'w. (typ. u. var. mestulutu Buchh. l'ts.). Elsemlaher. Gek. 1887 von demselben.

\section{Genus L. Leptophis Bell.}

1. Leptoplis uccidentalis (Gthr.).

Boulenger, Cat. II pag. 111, Taf. 3, Fig. 2.

8111,1 a Erw. San José, Costa Rica. Gesch. 1893 ron Carl Fleischmann, Guatemala. 
2. Leptophis liocercus (Wied).

Bonlenger, Cat. II pag. 113.

8113 a. Jung. Trinidal. Gesch. 1879 ron Karl Knoblanch, hier.

8113 b. $2 \mathrm{crw}$. (Rïppell. ('at. III T 1 a-b als Dendrophis ahactulla). Itheos,

Brasilien. Gesch. 1838 ron Gebr. Koch, hier.

8113 c. Erw. (Riippell, (at. III T 1 c als 7 . ahatulla). Surinam. Gesch. 1836 ron Dr. merl. Sichel, hier.

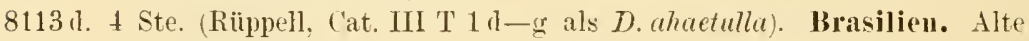
Sammlung $18+5$.

8113 e. Erw. Trinidad. Gesch. 1893 ron F. W. Trich und R. R. Mole, Port of Spain.

8113 f. Erw. (mit rerletztem Kopf). Renedios in $600 \mathrm{~m}$. U. S. Columbia. Gek. 1897 ron I'rof. Dr. Fritz Regel, Jena.

Genus LI. Uromacer D. B.

1. Uromacel catesbyi (Schlg.).

Boulenger, (at. II pag. 11 .

8115́a. Erw. Cap Haytien, Haiti. Gek. 1888 rom Naturh. Institut Linnaea, Berlin.

2. Uromaceroxyriynchus D. B.

B oulenger, Cat. Il pag. 116.

8117 a. 2 erw. Cap Haytien, Haiti. Gek. 1888 rom Naturh. Institut Linnaea, Berlin.

Gemus LII. Hypsirhynchus Gther.

1. Hypsirlyehus ferox Gthr.

Boulenger, Cat. II pag. 117, Taf. 6, Fig. 1.

8118 a. Erw. Cap Haytien. Haiti. Gek. 1888 rom Naturh. Institut Linnaea, Berlin.

8118 b. Erw. San Domingo. Get. 1893 rom Naturhist. Verein in Augsburg.

\section{Genus LIII. Dromicus Bibr.}

1. Dromicus chamissoi (IVgm.).

Boulenger, Cat. II pag 119.

8119 a. Erw. Chile. Gesch. 1874 ron Bergingenienr Eich, Geisnidda (Oberhessen1. 
2. Dromicus antillensis (Schlg.).

Boulenger, Cat. II pag. 123.

8123 a. Erw. u. jung. St. Thomas. Gesch. 1881 von Karl Knoblauch, hier. 8123 b. 3 erw. Elsendaher. Gesch. 1882 von demselben.

3. Dromicus rufiventris D. B.

B oulenger, Cat. II pag. 124.

8124 a. Erw. (Rüppell, Cat. III P 8 a als Herpetodryas sp.), Westindien. Alte Sammlung $18+5$.

4. Dromicus anomalus (Pts.).

Boulenger, Cat. II pag. 125.

8125̌ a. Halbw. Cap Haytien, Haiti. Gek. 1888 rom Naturlı. Institut Linnaea, Berlin.

\section{Genus LIT. Liophis Wgl.}

1. Liophis taeniurus Tschudi.

Boulenger, Cat. II pag. 130.

8130 a. Erw. Altaquer bei Barbacoas, Siidwest-U.S. Columbia. Gesch. 1885 ron Konsul F. C. Lehmann, Popayán.

2. Liophis albiventris Jan.

Boulenger, Cat. II pag. 130.

8130,1 a. Jung. Peru. Gesch. 1863 von Feldmarschall Philipp Brann, Lima. 8130,1 b. Erw. Ecllador. Gek. 1877 von Hamburg.

3. Liophis poecilogylus (Wied).

Bonlenger, ('at. II pag. 131.

8132 a. 3 erw. (Rïppell, ('at. III G fic-e als Coronella merremi). Buenos Aires. Gesch. 1840 ron W. A. Bermus, lier.

8132 b. 3 elw.. 6 jung. Rio Grande do Sul, Sül-Brasilien. Gesch. 1872-

1886 von Oherlehrer Dr. Finger: hier, nut Prof. Dr. H. von Thering. daselist.

8132 c. 2 erw., 2 jung. Săo Paulo, Brasilien. Gek. 1881 von .Josef Dusclianek, hier.

8132 d, 2 Junge. Buenos dires. Gesch. 1886i ron Prof. Dr. O. Boettger, hier. 8132 e. Halbw. Siid-Amerika. Gek. 1887 ron C. A. Pölıl, Hamburg.

8132 f. 2 Junge (Rüppell, Cat. III G 5 a-b als C.dolicta). Brasilien. Gesch.

1840 von Schïff Dr. Carl von Heyilen, hier.

$8132 \mathrm{~g} .3$ halbw. (Riippell, ('at. III G $5 \mathrm{c}-\mathrm{e}$ als (! doliutc). Siil-Brasilieu. (iesch. 1828 rom Herzog von Lenchtenherg. 
8132 h. 3 erw. (Riippell, Cat. III G $5 \mathrm{f}-\mathrm{h}$ als $C$ doliata), Siid-Brasilien. Gesch. 1838 ron Schöff Dr. ('arl ron Heyden. hier.

8132i. Jung. São Paulo, Brasilien. Gek. 1876 von Prof. Cárlos Miiller: daselbst.

$8132 \mathrm{k}$. Erw. Ebendaher. Gesch. 1890 ron Prof. Dr. O. Boettger, hier.

81321. Erw. Central-Brasilien. Gesch. 1896 ron Konsul Guido von Sclıröter, San José (Costa Rica).

4. Liophis melanotus (Shaw).

B o u l onger. Cat. II pag. 134 .

8134 a. Erw. Trinidad. Gesch. 1893 ron F. IV. Urich und R. R. MLole. Port of Spain.

8134 b. Erw. u. 3 jung. Carícas. Venezuela. Gek. 1897 ron Ingen. Müller. hier.

5. Lioplis almadensis (Wgl.).

Boulenger, Cat. II pag. 134: Boettger. Zeitschr. f. 1. ges. Naturw. Bd. ว̌. 1885 pag. 228.

8135̃a. 6 Ste. Săo Paulo, Brasilien. Gek. 1876-81 ron Prof. Cárlos Müiller und Josef Duschanek. daselbst.

8135) b. 3 erw, Rio Grande do Sul, Siid-Brasilien. Get. 1888 von Prof. Dr. H. von Thering. daselbst.

813j c. Erw. Matogrosiso, Brasilien. Gesch. 1893 ron Гir. Dr. Cárlos Berğ. Buenos Aires.

813ă d. Erw. Stadt Rio Frande do Nul. Gek. 1886 ron Prof. Dr. H. ron Ihering, daselbst.

813je. 2 erw. Central-B asilien. Gesch. 1896 ron Konsul Guido ron Schriter. San José (Costa Rica).

$$
\text { 6. Liophis typhlns (L.). }
$$

Bonlenger: Cat. II pag. 136; Renb. Mus. Senck. Pul. 1. 1834 pag. 144 (Coluber albicentris); Boettger. Zeitschr. f. d. ges. Naturw. Bd. 58. 1885 pag. 229.

8136 a. Jung (Riippell. Cat. III O 16 a als (. albicentris Rss.). Brasilien. Alte Sammlung 1845 .

8136 b. Erw. Brasilien. Gek. 1877 ron Hamburg.

8136 c. Erw. Central-Brasilien. Gesch. 1896 von Konsul G. von Selurïter. San José (Costa Rica).

7. Liophis epinephelus Cope.

Boulenger. Cat. II pag. 137.

8136.1 a. Erw. Cuenca, Ecuador. Gesch. 1896 von Konsul F. C. Lehmam, Popayín. 
8. Liophis reginae (L.).

Boulenger, Cat, II pag. 137.

8137 a. Erw. Puerto Cabello, Venezuela. Gesch. 1893 von Konsul F. MauB, laselbst.

8137 b. Erw. Talenicia, Venezuela. Gesch. 1894 ron F. Wr. Urich und R. R. Mole, Port of Spain.

8137 c. Erw. Trinidad. Gesch. 1893 ron denselben.

8137 d. Jung (Rüppell, Cat. Ill G 3 f als Coronellı). Surinam. Gesch. 1832 ron Dr. merl. Sichel, hier.

8137 e. Jung. Trinidad. Gesch. 1894 ron F. W. Urich und R. R. Mole.

8137 f. Erw. (Riippell. Cat. III G 3 a als Coronella). Surinam. Gesch. 1832 ron Dr. med. Sichel, hier.

8137 g. 4 erw. (Riippell. Cat. III G 3 b-e als Coronella). Brasilien. Alte Samming 184 o.

$$
\text { 9. Liophis juliae (Cope). }
$$

Boulenger, Cat. II pag. 139.

8139 a. Erw. Insel Iominica, Westindien. Get. 1893 vom British Iruseum (X. H.). London.

10. Liophis parifrons (cope).

Boulenger, Cat. II pag. 141.

8141 a. Erw, u. halbw. Cap Haytien, Haiti. Gek. 1888 vom Naturh. Institut Linnaea, Berlin.

11. Liophis melanostigma ( $\mathrm{Tgl}$.).

Boulenger, l'at. II pag. 142.

8142 a. Kolf. Rio Grande do Sul, Siid-Prasilien. Gek. 1886 von P'rof. Dr.

H. von thering, daselbst.

\section{Gemus LV. Xenodon Boie.}

1. Senodon colubrius Gthr.

Boulenger, Cat. II pag. 146.

8146 a. Halbw. (Rüppell. Cat. III H 2 a als (J)his rhululocephalus). Brasilien. Alte Sammlung $18+5$.

2. Xenodon neuwiedi Gthr.

Boulenger, Cat. II pag. 148.

8148 a. Erw. Rio de Janciro. Gesch. 1897 ron Prof. Dr. O. Poettger, hier. 
3. Xenodon severus (L.).

Boulenger. Cat. II pag. 149.

8149 a. Halbw. Puerto Cabello, Veneznela. Gesch. 1894 ron Konsul F. Mauß. daselbst.

8149 b. Jung. Sorata, Bolivia. Gesch. 1894 von Dr. Heinrich Lenz, Luibeck. 8149 c. Erw. Surinam. Gek. 1846 von Stuttgart.

8149 d. Jung. Puerto Cabello. Gesch. 1893 von Konsul F. Mauß, daselbst. 8149 e. Erw. (Riippell, Cat. III H 1 a als Ophis). Surinam. Gesch. 1839 ron Dr. med. Sichel, hier.

8149 f. Erw. Carácas, Venezuela. Gek, 1897 von Ingenieur Müller; hier.

\section{Xenodon merremi ( $\mathrm{Wgl}$.).}

Boulenger, Cat. II pag. 150; Boettger, Zeitsclur. f. ‘l. ges. Naturw. Bd. 58, 1885 pag. 232 (secerus).

8150 a. Halbw. São Paulo, Brasilien. Gesch. 1890 von Prof. Dr. O. Boettger, lier.

8150 b. 3 erw.. 1 jung. Rio Fraude do Sul, Sücl-Brasilien. Get. 1886-8 8 von Prof. Dr. H. von Ihering, daselbst.

8150 c. 2 erw. São Panlo. Gek. 1876 von Prof. Cárlos Mialler, tlaselbst.

8150 d. 2 halbw. Ebendaher. Gek. 1881 von Josef Duschanek. hier.

8150 e. Erw. Corrientes, Rep. Argentina. Gesch. 1893 von Dir. Dr. ('árlos Berg, Buenos Aires.

8150 f. Halbw. Central-Brasilien. Gescli. 189; von Konsul Guilo von Schröter, Sall José (Costa Rica).

\section{Genus LVI. Lystroplis Cope.}

1. Lystrophis dorbignyi (D. B.).

Boulenger, (at. II pag. 151; Boettger, Zeitschrift f. d. ges. Yaturw. Bul. 58, 1885 pag. 233 (Heterodon).

8151 a. Erw. Rio Grande do Sul, Sïl-Brasilien. Gesch. 1873 von Oberlehrer 1)r. Finger. hier.

2. Lystrophis histricus (Jan).

Boulenger, ('at. II pag 152.

8152 a. Erw. Rio Graude do Aul, Sür-Brasilien. Gesch. 1873 ron Oberlehrer Dr. Finger, hier. 


\section{Genus LIVII. Heterodon Latr.}

1. Heterodon platyruhins Latr.

Boule nger, Cat. II pag. 155.

815i) a. 4 Ste. (Riippell, (at. III J 1 b-e), Verein. Staateu. Gescl. 1836 von Dr. med. Reuß mi WV. Engelmann, St. Louis.

8155 h. 3 halbw. Verein. Staaten. Gesch. 1874 von der Nenen Zoolog. Gesellschaft, hier.

81в̈ с. Erw. o. Texas. Gesch. 1897 von Dr. merl. Arthur Hanan, st. Gallen.

2. Heterodon simus (L.).

B oulenger, ('at. II pag. 156.

8156a. Erw. (Rüppell, Cat. III J 1 a als platyrrhinus). Verein. Staaten. Gesch. $18 \pm 0$ von Dr. med. Eduard Rïppell. hier.

Gemus LIIII. Aporophis Cope.

1. Aporophis lineatus ( $\mathrm{I}$.).

Boulenger, l'at. II pag: 158.

8159 a. 3 Ste. (Riippell, Cat, III 1'5a-c als Herpetodryas). Brasilien. Alte Sammlung 1845 .

8158 b. Erw. (Riippell. ('at. IlI f' 5 d als Herpetodryas). Suruam. Gesell. 1836 ron Dr. med. Sichel, hier.

8158c. Halbw. Rio Branco. Gek. 1897 von Greorg Hïbner, Dresiten.

8158 c. 2 halbw. Baranquilla im Magdalenathal, U. S. Columbia. Crek. 1897 von Prof. Dr. Fritz Regel, Jena.

2. Aporophis flavifrenatus (Cope).

B oulenger. ('at. II pag. 158 .

8158,1 a. Erw. Rio Grande do Sul, Siid-Brasilien. Gek, 1886 ron Prof.

[)r. H. von Thering, daselbst.

\section{Genus LIX. Rhadinaen Cope.}

1. Rhadinaea breviceps (Cope).

Boulenger, ('at. II pag'. 164.

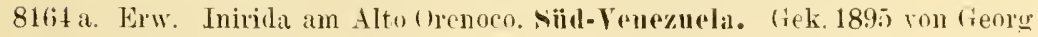
Hiibner. Dresslen. 
2. Rhadinaea anomala (Gthr.).

Bonlenger, Cat. II pag. 165.

8165 a. Erw. ठ. Rio Grande do Sul, Siid-Brasilien. Gesch. 1873 von Oberlehrer Dr. Finger, hier.

8165 b. Erw. La Plata, Rep. Argentina. Gesch. 1894 ron Dr. Jean Valentin, Buenos Aires.

3. Rhadinaea sagittifera (Jan).

B oulenger, Cat. II pag. 165.

8165,1 a. Erw. Buenos Aires, Rep. Argentina. Get. 1891 rom Yaturh. Mus. Offenbach.

4. Rhadinaea cobella (L.).

B o ul enger, Cat. II pag. 166.

8166a. Erw. Trinidad. Gesch. 1895 von F. W. Urich und R. R. Mole, Port of Spain.

8166 b. Erw. Ebendaher. Gesch. 1893 ron denselben.

8166 c. 3 Junge (Riippell. Cat. III G $4 \mathrm{~d}-\mathrm{f}$ als Coronella). Surinam. Gesch. 1838 ron Dr. med. Sichel, hier.

8166 d. 3 erw. (Riippell, Cat. III G 4 a-c als Coronella). Nurinam. Gesch. 1838 ron demselben.

8166 e. 3 erw. (Riippell, Cat. III G $4 \mathrm{~g}$-i als Coronellu). Nurimam. Gesch. 1838 von demselben.

var. taeniogastra Jan.

B oulenger, l. c. pag. 167.

8167 a. 2 Junge. U. S. Columbia. Gesch. 1894 vou Konsul F. C. Lehmann, Popayán.

5. Rhadiuaea merremi (Wied).

B o u lenger, Cat. II pag. 168; Reı B, Miıs Sesck. Bd.1, 1834 pag. 145 Taf. 8, Fig. 1 (Coluber bicolor).

8168a. 2 erw. (Rïppell, Cat. JII G $6 \mathrm{a}-\mathrm{b}$ als Coronella). Brasilien. Get. 1838 von Graf Jenison, Heidelberg.

8168b. Erw. Brasilien. Gesch. 1877 von Prof. Dr. Ferdinand Richters, hier. 8168c. \& Ste. Sĩo Paulo, Brasilien. Gek. 1876 und 1881 ron Prof. Cárlos Miuller, daselbst, und Josef Duschanek, lier.

8168 d. 2 erw. (Originale von Coluber bicolor Rss.: Rüppell, Cat. III G 5* $\mathrm{a}-\mathrm{b}$ als Coronella doliata var.). Ilheos, Brasilien. Gesch. 1830 von Gebr. Koch, hier. 
6. Rhadinaea fusca (Cope).

Bo ulenger. Cat. II pag. 169.

8169 a. Erw., halbw. и. 22 Junge. Rio Grande to Sut, sïd-Brasilien. Gek. 1886 ron Prof. Dr. H. ron Thering, daselbst.

7. Rhadinaea jaegeri (Gthr.).

Boulenger, Cat. II pag. 170, Taf. 7, Fig. :3.

8170 a. 2 erw. Rio (xraude do Sul, Sïr-Brasilien. Get. 1888 von Prof. Dr.

H. von Thering, daselbst.

8170 b. 2 erw. Ebendaher. Gek. 1886-87 von demselben.

8170 c. உ2 erw. Săı Paulo, Brasilien. Gek. 1881 von Josef Iuschanek, hier.

8. Rhadinaea affinis (Gthr.).

B o nlenger, Cat. II pag. 172.

8172.1 a. Kopf u. halbw. Rio Grande do sul, sïl-Brasilien. Gek. 1886 von Prof. 1)r. H. von lhering. laselbst.

9. Rhadinaea undulata (Wied).

Boulenger, ('at. II pag. 174; Bo ettger. Ber. Senck. Nat. Ges. 1888 pag. 195) (Coronella taeniolata).

8174,1a. Erw. Mapiri, Nebenthß des oberen Beni. Bolivia. Gesch. 1888 von Ferdinand Emmel, Aresuipa.

10. Rhadinaea occipitalis (Jan).

Boulenger, ('at. II pag. 175; Boettger, Zool. Anzeiger 18!1 pag. 395 (Diomicus miolevis).

817ē,1 a Erw. Santos, Nord-Brasilien. Get. $188+$ rom Naturh. Museum in Hamburg.

817..1b. Erw. Central-Brasilien. Gesch. 1896 ron Konsul Guirlo ron schröter, San José (costa Rica).

8175.1 c. Erw. Sorata. Bolivia. (iesch. 18!7 ron Prof. Dr. (1)kar Boettger. hier.

11. Rhadinaea rittata (Jan).

Boulenger, l'at. II pag. 178.

8178 a. Halbw. Vera ruz. Mexiko. Gek. 187t ron Hamburg. 


\section{Rhadinaea kinkelini n. sp.*)}

8178,1 a. Erw. Iatagalpa, Nicaragua. Gesch. 1897 ron Apotheker Adolf Kinkelin, Nïrnberg.

\section{Gemus L.Y. Urotheca Bibr}

\section{Urotheca bicincta (Herm.).}

Boulenger, Cat. II pag. 184.

8184 a. Erw. J. Weg ron Guaramaco mach San Fernando, Alto Orenoco, siid-Yenezuela. Gek. 1845 ron Georg Hiibner, Dresden.

*) Nächstrerwandt der mexikanischen Rh. vittata (Jan), aber mit kiirzerem Schwanze unıl anderer Färbung. AnBerdem sind die vorderen Kinnschilder viel länger als die hinteren, was bei Rh. vittata umgekehrt ist. Färbung ähnlich łer Rh. godmani (Gthr.) ans Gnatemala. - Oberkieferzähne 16. nach hinten allmählich größer werdend, die hinteren nicht zusammengedrüclt. Ange ziemlich klein. Schnanze nicht hervorragend; Rostrale anderthalbmal so breit wie hoch, ron oben eben noch sichtbar. Internasalsutur kurz, kaum halb so lang wie die Praefrontalsutur; Frontale so lang wie sein Abstand rom Schnanzenende, etwa zwei Drittel Io lang wie die Parietalen. Frenale etwas länger als hoch; ein Praeocnlare, das das Frontale nicht erreicht; 2 Postocularen; Temporalen $1+2$. Acht Supralabialen. das vierte und fünfte ans Ange tretend, das sechste an höchsten. Von den Infralabialen treten vier an die vorderen Kinnschilder, die sehr erheblich länger sind als die hinteren.

Sehuppenformel: Squ. $17 ;$ G. ${ }^{3}{ }_{3}$, V. 146, A. ${ }^{3}{ }_{1}$, Sc. ${ }^{72}, 72+1$.

Braun wit dunklerer, eine Schuppenreihe breiter, an der Schnauze beginnender Kïckenlinie und je zwei schmalen; ebenfalls dunkeln Seitenlinies. Die obere Seitenlinie wird nach oben ron einem lsellen, eine ha!be Schnppenreihe breiten Längsstreifen begrenzt. Ein schmales gelbliches Querband, das nur in der Hitte durch den dunkeln Spinalstreifen unterbrochen ist, liegt quer iiber dem Nacken. Das Rostrale ist weißgelb mit einer $\wedge$-förmigen schwarzen Makel; die Lippenschilder sind, mit Ansnahme des letzten, ganz dunkeln, weißgelb mit breiten schwarzen Säumen. Auf den Parietalen zeigen sich zwei dicht bei einander stehende weiße Fleckchen. Die Unterseite ist scharf abgesetzt lehmgelb, nur die Schuppen der Kopiunterseite zeigen grauliche Schnppenränder; die seitlichen Sänme der Ventralen sind mit einer feinen graulichen Längslinie, wie eine solche auch zwischen den beiden runkehn Seitenstreifen zu beobachten ist. gezeichnet.

II a Be: Totallänge 358 , schwanzlänge $102 \mathrm{~mm}$.

Fundort: Matagalpa, Nicaragua, nur in dem schön erhaltenen Stück bekannt, das wir von Herrn Apotheker Adolf Kinkelin in Nürnberg, dem zu Ehren die Art benannt wurle, zum Geschenk erhalten haben. 


\section{Genus LXI. Fleischmannia nov.}

Oberkiefer mit 12 schlanken, bis zum letzten in gleichen Abständen stehenden Zïlmen, die letzten zwei etwas größer, cylindrisch, ungefurcht. Unterkiefer mit 17 Zähnen, die vordersten drei etwas kleiner, die mittleren allmählich größer werdend, die der zweiten Kieferhälfte am kleinsten, merklich an Größe abnelmend. - Kopf breiter als der Hals, in der Wangengegend deutlich angeschwollen. Ange ziemlich grob, mit runder Pupille. Obere Kopfschilder regelmäBig; Nasenloch seitlich, in der Vorderhälfte eines großen, einfachen Nasale. Ein Frenale fehlt. Körper mäßig verlängert, walzenförmig; Schuppen glatt, ohne Endporen, in 17 Reihen; Ventralen verrundet. Schwanz lang; Subcaudalen in zwei Reihen. - Central-Amerika.

Verwandt den Gattnngen Trimetopon Cope und Hydromorphus Pts., aber mit doppelten Praefrontale. Ihre Physiognomie erinnert, abgesehen von der runden Pupille, etwas an die von Psammodynastes.

Hierher als einzige Art:

\section{Fleischmannia obscura n. sp.*)}

8185 a. Erw. San José, Costa Rica. Gesch. 1893 von Carl Fleischnann, Guatemala.

*) Kopf oben flach, zwischen den Augen leicht ausgehöhlt. Schnauze sehr kurz, nicht ganz anderthalbmal so lang wie das Auge, kegeliörmig verrundet, etwas vorgezogen und schief abgestutzt, mit deutlichem Canthus rostralis. Auge etwas größer als sein Abstand von der Maulspalte. Rostrale etwas breiter als huch, ron oben kaum sichtbar; Internasalen $2 / 3$ so lang wie die Praefrontalen; Frontale doppelt so lang wie in der Mitte breit, länger als sein Abstand von der Schnauzenspitze, kürzer als die Parietalen. Ein Frenale fehlt; ein Praeoculare, das die Oberseite des Kopfes nicht oder kaum erreicht; Temporalen $1+2.7$ Supralabialen, von denen das dritte und vierte ans Auge stoßen. 4 Infralabialen in Berührung mit den vorderen Kinnschildern, die wesentlich kïrzer sind als die hinteren.

Schuppenformel: Squ. 17 ; G. ${ }^{2}{ }_{1}$, V. 123, A. ${ }^{1}{ }_{1}$, Sc. ${ }^{79}{ }_{79}+1$.

Oberseits dunkler. unterseits heller lederbriun, die Banch- und Schwanzschilder mit dnnkleren Vurdersäumen und Kehle und Halsgegend anBerdem noch mit undeutlichen, dunkleren Längszeichnungen. Hintere Kopfhälfte heller, ledergelb, gegen das Braun des Nackens ziemlich scharf $\wedge$-förmig abgegrenzt. Vom Ange zieht gegen die Temporalgegend ein breiter, dunkelbranner, beiderseits hell eingefaßter, schiefer Streifen.

II a B e: Totallänge 302 , Schwanzlänge $99 \mathrm{~mm}$.

F und ort: San José, Rep. Costa Rica, nur in einem tadellosen Stïcke von Herrn Cárlıs Fleischmann in Guatemala, dem zu Ehren ich die crattung benannt habe, gesammelt und eingesandt. 


\section{Genus LXII. Hydrops Wgl.}

1. Hydrops triangularis (Wgl.).

Bonlenger: ('at. II pag. 187.

8187 a. Jung (schleclit gehalten). Cindad Bolivar. Venezuela. Gesch. 1894 ron Apotheker Theod. Liining daselbst dmrel Apotheker Dr. Ang. Jassoy. hier.

818т b. Erw. u. halbw. (Rüppell. C'at. III X ja-b als Homalopsis martii). Brisilien. Alte Sammlung 1845.

8187 c. Erw. Surinam. Gesch. 1893 von F. W. Urich und R. R. Jole. Port of spain.

\section{Genus LXIII. Coronella Laur.}

1. Colouella austiaca Lam.

Boulenger, Cat. II pag. 191; Boet t erer. Zeitschr. f. d. ges. Naturw. Bil. 49. 1877 pag. 286 mul in Rarlde's Faum. n. Flora ('asp-Geb. 1886 pag. 67.

8191 a. Erw. Jngenhein an der Bergstrabe. (iesch. 1894 von Kanfm. Hernn. Wichmanm. hier.

8191 b. Erw, Soden in Tanmus. Gesch, 1844 ron Geh. Hofrat Dr. merl. sal. Stiebel. liier.

8191 c. Erw. u. halbw. Altkönị̆ in Taunus. (iesch. 1876 von Prof. Lr. 0. Boettger, hier.

8191 d. Erw. Pror. Sachsen. Gesch. 1890 ron demselhen.

8191 e. Halbw. Thüringen, Gesch. 1871 von Irir. Dr. Theod. Geyler, hier.

8191 f. Erw. Orl), Kr. Gelnhausen, Hessen-Nassan. Gesch. 1891 ron (Oberlehrer .J. Blum. hier.

$8191 \mathrm{~g}$. Erw. Piisseburg bei Thiengen, hr. Wa'dshut. Barten. Gesch. 1891 von demselhen.

8191 h. Erw. Kobyllno, Oberschlesien. Gesch. 18si) ron Otto GollfuB. Halle (Saale).

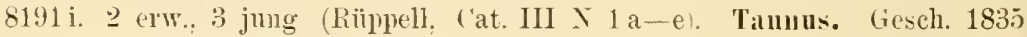
ron Scliöff Dr. Carl ron Heyrlen, hier.

8191 k. Erw. Weinlein a. l. Bergstraße. Gesch.18st von Albrecht Weis. hier.

8191 1. Erw. Jiiterbogk, Prov. Branlenburg. Gesch. 1891 ron Oberlehrer J. Blum. hier.

$8191 \mathrm{~m}$. 2 erw. Richisan im kïnthal, Ct. Glarus, Schweiz. (iesch. 1891 ron iemselhen.

8191 u. Erw. ㅇ, 3 Eier, 2 Junge. Frankfurter Wald. (iesch. 1849 von G. Kinch III, hier.

81910 . Erw. Falkenstein im Taunus. Gesch. 1893 ron harl Henrich. hier. 8191 p. Halbw. Branl. Sächsische Schweiz. Gesch. 1886 ron Alhr. Weis. 8191 q. Halbw. Geltschberg bei Leitmeritz. Nord-Böhmen. Gesch. 1886 ron demselben. 
$8191 \mathrm{r}$. Jung. Geierkogel in $800 \mathrm{~m}$ bei Graz, Steiermark. Gesch. 1886 von Hauptmaun-duditor Hipp. Tschapeck, Wien.

8191 s. Erw. Surlak, Krim. Gesch. 1883 von Staatsrat Otto Retowshi, Theodosia.

8191 t. Erw. Coruña, Nordwest-Spanien. Gesch. 1880 von V. Lopez Seoane, daselbst.

8191 u. Erw. (var. fitzingeri Bonap.). Mte. Rosso bei Syrakus, Sicilien. Gesch. 1886 ron Chefinspektor Carl Hirsch, Palermo.

8191 v. Erw. (dieselbe Varietät). Caltanisetta auf Sicilien. Gesch. 1886 von demselben.

2. Coronella amaliae (Bttgr.).

B o u l enger, Cat. II pag. 193; B oettger, Zool. Anzeiger 1881 pag. 570 und Abh. Senck. Nat. Ges. Bd. 13, 1883 pag. 98, Taf. 1, Fig. 1 (Rhinechis).

8193 a. Erw. Marokko. Erh. 1881 von der IV. Rüppellreise Dr. med. W. Kobelt's. Schwanheim (Main).

3. Coronella girondica (Daud.).

Boulenger, Cat. II pag. 194; Boettger, Abh. Senck. Nat. Ges. Bil. 9, 1873 pag. 150 und 10. Ber. Offenbach. Ver. f. Naturk. 1869 pag. 55 (laevis var. hispanica).

8194 a. Erw. Alpujaras, Sïl-Spanien. Gesch. 1868 von Major Dr. L. von Heyden, hier.

819ł b. Erw. Stadt Marokko. Gesch. 1872 von den Proff. Drr. Freih. C. von Fritsch uıd J. J. Rein.

8194 c. Erw. Tlemçen, Algerien. Erh. 1881 von der IV. Rüppellreise Dr. med. W. Kobelt's, Schwanheim (Main).

8194 d. Halbw. Iontpellier, Dép. Hérault. Gesch. 1887 von Joh. von Fischer, dasellust.

4. Coronella semiornata Pts.

Boulenger, Cat. II pag. 195; Boettger, Zool. Anzeiger 1893 pag. 117 (plumbiceps).

8195a. Erw. Moschi bei Tanga, Kilima-Njaro. Gesch. 1897 von Ingenieur Karl Nolte, daselbst.

5. Coronella getula (L.).

Bo uleuger, Cat. II pag. 197.

var. sayi Holbr.

B o u lenger. l. c. pag. 198; J a n, Icon. Ophid. Lief. 14, Taf. 5, Fig. 2.

8198 a. Erw. Siidliche Verein. Staaten. Gek. 1877 von Hamburg. 
8198 b. Erw. Ebendaher. (iesch. 1891 rom der Nenen Zoolog. Gesellschaft, hier.

S198 c. 2 erw. (Riippell, (at. 1 II P 9 a-l) als Herpelodryge?; Originale zu lan's oben citierter Abhildung). Ebendaher. fiesch. 1838 von Tr. med. Reuß und W. Engelmann, St. Lonis.

6. Coronella triangulum (Dand.).

Boulenger, ('at. II pag. 200.

8200 a. Halbw. Milwankee, Wisconsin. Gesch. 1893 von Bierloraner Rudolf Henrich, lier.

var. collaris Cope.

B 0 u l en ge r, l. с. pag. 201; .J a 11, Icon. Ophiıl. Lief. 17, 'Taf. 1, Fig. 3 (C. eximia).

8201 a. Erw. Siidwestliche Verein. Staaten. Gesch. 1891 ron Dr. A. Zipperlen, Cincimmati (Ohio).

8201 b. 5 Ste. (Riippell, ('at. III $09 \mathrm{a}-\mathrm{e}$ als Coluber guttatus; Originale zu Jan's oben citierter Abbildung). Verein. Staaten. Gesch. 1838 von Dr. merl. lieuf unil W. Engelmann, St. Louis.

7. Coronella micropholis (cope).

boulenger. ('at. II pag. 203 .

8:03 a. Erw. (var. A). Mexiko. Gesch. 1877 von l'rof. In. Ferdinand kichters. hier.

8. Colomella lunctata (L.).

Bonlenger, Cat. II pag. 206.

8206,1 a. 3 crw. Hilwaukee, Wisconsin. Gesch. 1893 ron Bicrlorauer Ludolf Henricli, lier.

\section{Genus LXII. Drepanodon Peracea.}

1. Drepanodon allomalus (Janl).

Boulenger, Cat. III pag. 639; Boettger, Zool. Anzeiger 1891 pag. 347 (Oxyrrhopus).

8212 a. Jung. Sorata. Bolivia. Gesch. 1891 ron Dr. Heinrich Lenz, Liibeck. 8212 b. Halbw. Ebendaher. Gesch. 1895 ron Prof. Dr. O. Boettger, hier. 
Genus I.IT. Cemophore Cope.

1. ('emoliola coccinea (Blmmenb.).

Bonlenger. Cat. II pag. 214.

8214 a. Halbw. Siidliche Verein. Staaten. Get. 1873 rom Naturh. Mlusem in Offenhach a. Ir.

8214b. Erw. Florida. Gesch. 1896 von Dr. 1. Zipperlen. ('incinnati (Uhio).

Genus LXVI. Simoles I). B.

1. Simotes purpurascens (Schlg.).

Bo ulenger', ('at. II pag. 218.

8218a. Erw. Tenggergebirge in $1200 \mathrm{~m}$, Ost-Jara. Gek. 1891 vol Hans Fruhstorfer, Berlin.

Var. labuanensis Gthr.

Boulenger, 1. с. pag. 219.

8219 a. Erw. Klang in Selangor, Malayische Halbiusel. Gesch. 1895 von Prof. Dr. 0. Boettger, hier.

8219 b. Erw. Atschin. Nord-Sumatra. Gek. 1880 von Komrad Kläsi, Zürich.

var. trinotata D. B.

Boulenger, 1. c. pag. 219.

8:19,1 a. Erw. Sultanat Deli, Nordost-Sinuatri. Gesch. 1879 von Wr. yon Schonler. Wieshaden.

2. Simotes albocinctus (Cant.).

Bonlenger, l'at. II pag. 220 .

8221 a, 2 erw. (var. (). Darjeeling. Ost-Hinalaya. Get. 1891 vom British

Musenm (X. H.). London.

3. Simotes formosanus Gthr.

Boulenger, (at. II pag. 22.2. Taf. 8. Fig. 2: Boettger', Ber. Senck. Nat. Ges. $189+$ pag. 133, Taf. 3, Fig. ㄴ (hainanensis).

822.2 a. Erw. Insel Hailıı, Sürt-('hina. Gesch. 1894 ron Bernh. Schmacker, Shanglai.

4. Simotes octolineatus (Schud.).

boulenger, Cat. Il pag. 2.24.

8224 a. Jung. Baramfluß in Nord-Borneo, Erh. 1895 von der liiippellreise Prof. Dr. W. Kiikentlials, Jena. 
8224b. Erw. Insel Bangka. Grek. 1881 ron Konrat Kläsi. Zuirich. 8.2 4 c. Erw. (var. B). Sultanat Deli. Norlost-Sima1ra. Gesch. 1887 ron Prof. Dr. Haus Schinz. Zürich.

8.24 4 . 3 erw. Buitenzorg, West-Java. Gesch. 1890 rom Dr. Adolf Strubell, Bonl1.

8224 e. Erw. (var. B). Deli, Nordost-Suma1ra. Gesch. 1891 ron Dr. merl. O. Benecke, daselbst.

S.2 tf. Erw. (var. B). Elhendaler. Gesch. 1894 ron Wwe. H. Krämer, hier. 8.2 24 g. Erw. (var. meyerinki Strlchr.). Suln-Inselu. Get. 1897 rom Naturhist.

Hofmusenm, Wien.

5. Simotes phaeuochalinus Cope.

B onl enger. ('at. II pag. 225.

8-25̄a. Halbw. Prov. Nueva Ecija, Luzon. Gesch. 1880 von Konsul Dr. O. Fr. ron Mocllendorff, Manila.

8.25 b. Erw. u. jung. Manila, Luzou. Get. 1891 von demselben.

8.25 c. Halbw. Insel samar. Giesch. 1897 von demselben.

6. Simotes signatus Gthr.

Boule $\mathrm{ng}$ er. ('at. II pag. 2266 .

8226 a. Halbw. (schlecht gehalten). sultanat Deli, Mordost-Sumatra. Gesch. 1891 von Fritz Beyschlag: daselbst.

7. Simotes alnensis (Shaw).

Boulenger. ('at. II pag. 229.

8229,1 a. Halbw. Ceylon. Gek. 1889 von Hans Fruhstorfer, Berlin.

\section{Genus LIVII. Oligodon Boie.}

1. ()ligodou bitoluuatus Boie.

$\mathrm{Boulenger,} \mathrm{Cat.} \mathrm{II} \mathrm{pag.} 237$.

8237 a. Halbw. (Varietät mit vier dunklen Rückenstreifen). Gunnng Pengalengan, West-Java. Gek. 1895 von Hans Fruhstorfer, Berlin.

8237 h. Erw. (Riippell, Cat. III C 1 a als Calamuria). Jara. Get. 183 rom Rijksmuseum in Leirlen.

8237 c. Halbw. Java. Gesch 1876 ron Major Dr. L. von Heyden, hier. 82:37 d. Erw. Rejch Jolo, Jara. Gesch. 1868 ron E. Lörsch, hier.

8237 e. Erw. Tenggergebirge in 0st-Jara. Gek. 1891 ron Hans Fruhstorier, Berlin.

8237f. Erw. (gestreifte Varietät). Gumung Tjisurupan, West-Java. Gek. 1893 ron demselben. 
2. Oligudon everetti Blgr.

Bonlenger, Cat. II pag. 239, Taf.11. Fig. 1.

S:3:1,1 a. Erw. Banjermasin, Siidost-Borneo. Gek. 1894 ron Wwe. H. Krieb. hier.

3. Oligodon templetoni Gthr.

Bonlenger. Cat. II pay.,241.

82+1.1 a. Erw. Ceylou. Gesch. 1846 ron H. Worms, hier.

4. Oligodon sublineatus D. B.

Bonlenger, Cat. Il pag. 242.

8242a. Eru. C'eylom. Gek. 1889 von Hans Frulstorfer', Berlin.

8242 b. 6 ste. Ehendaher. Gesch. 1846 ron H. Worms, hier.

82t2c Jung. Kandy. Ceylon. Gesch. 1890 von Dr. Arolf Strubell. Bonn.

5. Oligodon subgriseus D. B.

Bonlenger. Cat. II pag. 24:3.

8243 a. Erw. Nïd-Ceylon. Gek. 1889 rom Hans Fruhstorier, Berlin.

6. Oligodon ta eniul s F. Mïll.

Boulenger. Cat. II pag. 360.

8245,1 a. Erw. Rurukan. Celelses. Erh. 1895 von der Rïppellreise Prof.

Dr. IV. Kükenthals. Jena.

8245.1b. Halbw. Minaliassa. Celehes. Erlı, 189 o ron derselben.

7. Oligodon melanocephalus (Jan).

Bonlenger, ('at. II pag. 246: Boettger. Ber. Senck. Nat. Ges.

1878-79) pag. 60 (Homalosoma) nut 1479-80 pag. 139 (Rhynchocalcumus).

82tia. Erw. 1r. 3 Junge. Jaffa. Syrien. Ciesch. 1879 von Major 1)r. L. von Heyden. hier.

8246 b. Jung. Haiffa. Syrien. Gesch. 1881 ron Hams simon, Stuttgart.

\section{(ienus LIVIII. Leptocalamus Gthr.}

1. Leptocalamus torumatus Gthr.

Boulenger. Cat. II pag. 250.

8250 a. Erw. Baranquilla, I. S. Columbia. Gesch. 1894 von Prof. Dr. 0. Boettger, hier. 
Genus LXIX. Simophis Pts.

1. Simoplis rhinostoma (Schlg.).

Boulenger, Cat. II pag. 253.

8253 a. Erw. (Anpralabialen 8-8, das 4. und 5. ans Ange tretend). Lagoa

Santa, Brasilien. Gesch. 1886 von Prof. Dr. O. Boettger, hier.

8253 b. Erw. (Rïppell, Cat. III K 1 a als Rhinostoma proboscileum). Brasilien.

Get. 1830 rom Naturh. Hofmuseum in Wien.

Gemes LXI. Contia B. Gir.

1. Contia aestiva (L.).

Bonlenger, Cat. II pag. 258.

8258 a. 2 erw. (Rüppell, Cat. III P 2 a-b als Herpetodryas). Verein. Staaten.

Gesch. 1838 ron Dr. med. Reuß und W. Engelmanm, St. Louis.

2. Contia vernalis (Harl.).

Boulenger, Cat. II pag. 258.

8258,1 a. Erw. Baltimore. Get. 1873 vom Naturh. Mruseum in Offenbach (Hain). 8258,1 b. Elw. u. jung. Milwaukee, Wisconsin. Gesch. 1893 von Bierbrauer Rudolf Henrich, hier.

3. Contia decemlineata (D. B.).

Boulenger, Cat. Il pag. 260; Boettger, Ber. Senck. Nat. Ges. 1879-80 pag. 144 (Ablabes modestus vars.).

8260 a. 3 Ste. Jerusalem. Gesch. 1880 ron Hans Simon, Stuttgart.

8260 b. 4 Ste. (var. inornata Jan). Ebendalier. Gesch. 1880 von demselben. 8260 c. 4 ste. (2 typ. 2 rar. inomata Jan). Ebendaher. Gesch. 1881 von demselben.

\section{Contia collaris (Ménétr.).}

Bonlenger, Cat. II pag. 260; Boettger, Ber. Senck. Nat. Ges. 1890 pag. 294 (Ablutes morlestus) und sitz.-Ber. Akar. Berlin 1888 pag. 171 mml Ber. Senck. Nat. Ges. 189: pag. 147 (Cyclophis modestus).

860,1 a. Erw. n. halbw. Brumána im Libanon. Gesch. 1881 ron Hans Simon, Stnttgart.

8260,1 b. Erw. Sïdost-Karabagh, Russisch-Aruenien. Erh. 1890 von Dr. Jean Valentin's Reise.

8260.1 c. Halbw. Kodshory bei Tiflis, Trauskaukasien. Erh. 1890 ron derselben. 
var. semimaculata Bttgr.

B oul eng e r, l. c. pag. 261; B o et t g er, 15./16. Ber. Offenbach. Ver.

f. Naturk, 1876 pag. 58 , Taf. (Ablabes modestus var.).

8261 a. Erw. (Original). Chios. Gesch. 1862 ron Hofr. Dr. med. Pauli, hier.

var. punctatolineata Bttgr.

B oul enger, l. c. pag. 261; B o ettg er. Ber. Senck. Nat. Ges. 1892 pag. 147 (Cyclophis modestus var.).

8261,1 a. Erw. (Original). Russisch-Armenien. Erh. 1890 von der Reise Dr. Tean Valentin's in den Karabagh.

5. Contia rothi (Jan).

Boulenger, Cat. II pag. 262; Boettger, Ber. Senck. Nat. Ges. 1879-80 pag. 143 (Ablabes).

8262,2 a. Erw. u. jung. Haiffa, Syrien. Gesch. 1880 von Hans Simon, Stuttgart. 8262,2 b. Erw. u. jung. Jerusalem. Gesch. 1881 von demselben.

\section{Contia coronella (Schlg.).}

Bonlenger, Cat II pag. 264; Bo ettger, Ber. Senck. Nat. Ges. 1879-80 pag. 140 (Ablabes).

8264 a. Jung. Damaskus, Syrien. Gesch. $188+$ ron Hans Simon, Stuttgart. $826+$ b. 3 erw., 1 jung. Haiffa, syrien. Gesch. 1881 von demselben.

8264 c. Erw. u. jung. Beirut, Syrien. Gek. 1878 ron W. Schlïter, Halle (Saale). 8264d. Halbw. Syrien. Get. 1897 vom Natmrhist. Hofmuseum, Wien.

\section{Contia nasus (Gthr.).}

B o al lenger, Cat. II pag. 268.

8269 a. Erw. Jalisco in $8500^{\prime}$, Mexiko. Get. 1893 rom British Mruseum (N. H.), London.

\section{Genus LXXI. Homalosoma Wgl.}

1. Homalosoma lutrix (L.).

B oulenger, Cat. II pag. 274.

8274a. Erw. Kapland. Gek. 1887 von C. A. Pölıl, Hamburg.

$827+$ b. 4 erw. (Riippell. Cat. III C $2 \mathrm{a}-\mathrm{c}$ als Calamuric arctiventris). Kaplaul. Get. 1835 rom Rijksmuseum, Leitlen. 
Genus LIXII. Ablabes D. B.

1. Ablabes lierminae Bttgr.

Boulenger, (at. IlI pag. 643; Boettger, Zool. Anzeiger 189; pag. $27 \overline{7}$ und Ber. Offenbach. Ver. f. Naturk. 1895 pag. 110.

8.77 a. Erw. Yaeyama auf Mijakoshima, Nidlliche Liukin-Inselı. Gesch. 1895 von Bernhard Schmacker, shanghai.

2. Ablabes semicarinatus (Hallow.).

Boulenger, Cat. II pag. 278.

8278 a. 2 erw. Yaeyama auf Mijakoshima. Siidliche Liukiu-Inselı. Gesch. 1895 von Bernhard schmacker, Shanghai.

3. Ablabes major (Gthr.).

Boulenger. Cat. II pag. 279.

8279 a. 3 erw. Lüshan-Gebirge bei Kiukiang, Ost-('hina. Get. 1889 ron Pernharl schnnacker, Shanghai.

4. Ablabes tricolor (Schlg.).

B o u le nger, Cat. II pag. 281 .

8281,1 a. Erw. Tenggergebirge in $1200 \mathrm{~m}$. Ost-Java. (iek. 1891 ron Hans Fruhstorfer, Berlin.

8281.1b. Erw. Pontianak, West-Bormeo. Gesch. 1893 ron Barun Albert ron Reinach. lier.

5. Ablabes philippinus Bttgr.

B oettger, Zoolog. Anzeiger 1897 pag. 164.

8281.2 a. Erw. Insel ('nlion, Calamianes, Philippinen. Gesch. 1897 ron Konsul Dr. O. Fr. ron Moellendorff, Manila, und ()tto Koch. l'ebrí. 8281.2b. Erw. Insel Simar, Philippinen. Gesch. 1897 ron denselben.

$$
\text { 6. Ablabes rappi Gthr. }
$$

Boulenger, ('at. II pag. 282.

8282,1 a. Erw. Darjeehing, Ost-Himalaya. Get. 1891 rom British Museum (X. H.). Lonrlon.

\section{Ablabes baliodirus (Boie).}

B oulenger, ('at. II pag. 283.

8283 a. Erw. Buitenzorg, West-Ja ra. Gesch. 1890 ron Dr. A. Strubell. Bonn. 82833 b. Erw. ठ․ Tenggergebirge in $1200 \mathrm{~m}$, 0st-Java. Gek. 1891 ron Hans Frulistorfer, Berlin. 
8283c. Erw. West-Jara. Gek. 1893 von demselben.

8283 d. Erw. Sultanat Deli, Norlost-Sumatra. Gesch. 1891 ron Fritz Beyschlag, daselbst.

8. Ablabes longicauda Pts.

B oul enger, Cat. II pag. 284.

8284,1 a. Erw. Sultanat Deli, Nordost-Sumatra. Gesch. 1894 ron Wwe. H.

Krämer, hier.

\section{Genus LXXIII. Grayia Gthr.}

1. Glayia smythi (Leach).

B oulenger, Cat. II pag. 287, Taf. 13, Fig. 3; Boettger, Ber.

Senck. Nat. Ges. 1887-88 pag. 51 (trianguluris).

8286 a. Jung. Boma, Unter-Congo. Gek. 1887 von Paul Hesse, Venedig.

8286 b. Erw. Banana, Unter-('ongo. Gek. 1887 ron demselben.

\section{Genus LXXIV. Abastor Gray.}

1. Abastor erythrogrammus (Daud.).

Boulenger, Cat. II pag. 290.

8290 a. Erw. Verein. Staatell. Gesch. $187 t$ von der Nenen Zoolog. Gesellschaft, hier.

\section{Genus LXXI. Petalognathus D. B.}

1. Petalognathus nebulata (L.).

Boulenger, Cat. II pag. 293; J a n, Icon. Ophid. Lief. 37, Taf. 5, Fig. 3 (Leptognathus).

8293a. \& Ste. (Rïppell, Cat. III \ 7 a-d als Dipsas; Originale zu .Tan's ohen citierter Abbildung). Surinam. Alte Sammlung $18 \pm \check{\text { o }}$

8293 b. Erw. Nord-Brasilien. Gesch. 1896 ron Prof. Dr. O. Boettger, hier. 8293 c. Erw. Trinidad. Gesch. 1893 ron F. W. Urich und R. T. Mole. Port of Spain.

8293 r. Erw. Tropiscltes Mexiko. Gek. 1846 ron H. Finck, Ludwigsburg.

\section{Genus LXXTI. Atractus Wgl.}

1. Atractus badius (Boie).

Bo ulenger, ('at. II pag. 308: Boettger. Ber. Senck. Yat. Ges. 1888 pag. 192 (Geoplis).

8308 a. Erw. Sorata, Bolivia. Gesch. 1891 ron Dr. Heinr. Lenz, Lüheck.

8308 b. Erw. Mapiri, Nebenfluß des oberen Peni, Bolivia. Gesch, 1888 von Ferdinand Emmel, Arequipa. 
2. Atractus torquatus (D. B.).

Boulenger, cat. II pag. 309.

8309 a. Erw. (Riippell. Cat. III X jo als Homulopsis martii). Surinam. Alte Sammlung 18t5.

3. Atractus lehmanni n. sp. *)

8:310)a. 4 erw.. 2 jung. Cnenca, Ecuador. Gesch. 1894-96 ron Konsul F. C. Lehmann, Popayán.

4. Atractus reticulatus Blgr.

Boulenger, Cat. II pag. 311, Taf. 15, Fig. 3.

8311 a. Erw. J. Central - Brasilien. Gesch. 1896 ron Konsul Guido ron Schröter, San .Tosé (Costa Rica).**)

*) Verschieden von dem nahe verwandten A. crassicuudatus (D. B.) durch das Frontale, das breiter ist als lang und riel kïrzer als sein Abstand vom Schnauzenende, durch den Kontakt ron jederseits 4 oder 5 Infralabialen mit dem Kinnschilderpaar und lurch einfarbig schwarze, nicht gelblich gefleckte Oberseite und helle Schwanzunterseite. - Schnanze ziemlich stumpf. Rostrale klein, fast so hoch wie breit, von oben gerade noch sichtbar. Internasalen sehr klein; Praefrontalen so lang wie breit: Frontale breiter als lang, viel kürzer als sein Abstand ron der 'clmanzenspitze, nur etwa halb so lang wie die Parietalen. Frenale nicht mehr als zweimal so lang wie hoch: 2 Postocularen; Temporalen $1+2$. Sieben Supralabialen. von denen das dritte und rierte das Ange beriihren; vier oder fïnf Infralabialen in Berïhrung mit dem einzigen Paar Kinnschilder. die mäßig groß unı von dem Symphysale getrennt sint.

schuppenformeln: ठ Sigu. 17; G. 3. V. 142. A. 1 , Sc. ${ }^{27} ; 27+1$.

.. 17; . 4. . . 143. . 1 . , ${ }^{26 / 26}+1$.

. $, 17:, 5, \quad, 144, .1 . \quad .{ }^{25} / 25+1$.

ㅇ $" 17 ;, 4,, 148 ., 1 .,{ }^{20} / 20+1$,

\# $17:, 6, \ldots 148, \pi 1, \quad, 21 / 21+1$.

$\eta, 17 ;, 5, \pi 153, .1, \quad{ }^{20} / 20+1$.

Oberseits einfarbig rlunkel schwarzbram, äußerste Seitenscluppenreihe rötlichweiß. Lnterseits größtenteils schwarz mit rötlichweißen, meist quadratischen Flecken ; Kinn- nnd Schwanzunterseite größtenteils rötlichweiß. Junge Stiicke zeigen ein in der Mitte unterbrochenes rötlichweißes Halshand und je finen hellen Flecken auf den Internasalen.

II a Be: ¿ Totallänge 295 , schwanzlünge $33 \mathrm{~mm}$,

$$
\text { 요 } \pi 328,32 n \text {. }
$$

Fundort: Bei c'nenca in Ecuador von Herrn F. C. Lehmann. Konsul des Dentschen Reiches, gesammelt und in 6 Exemp'aren dem Senckenbergischen Inseum zum Geschenk ïherwiesen.

**) Das rorliegende $\delta$ hat ile Schuppenforme1: Squ. 15: G. 2, V. 141, A. 1. Se. ${ }^{31}{ }^{31}+1$ und unterscheidet sich auch dureh Pholidose nnd Färbung 
5. Atractus emmeli (Bttgr.).

Boulenger, Cat. II pag. 311; Boettger, Ber. Senck. Nat. Ges. 1888 pag. 192, Fig. (Geophis).

8311.1 a. 2 erw. (Originale). Mapiri, Nebentub des oberen Beni, Bolivia. Gesch. 1888 son Ferdinand Emmel, Arequipa.

$8: 311.1$ b. Erw. Sorata, Bolivia. Geseh. 1891 ron Dr. Heinrich Lenz, Lïbeck.

\section{Atractus trilineatus $\mathrm{Wg}$.}

Boulenger. ('at. II pag. 312.

8:312 a. Jung. 'T'rinidad. Gesch. 1894 vou F. W. Trich nud R. R. Mole, Port of Spain.

8:312 b. Erw. (Rïppell, Cat. III ('3 a als Culcmaria terlineutu). Surinam. Get. 1838 vom Rijksmuseum in Leiden.

8:312 e. :3 erw. Trinidad. Gesch, 1893 von F. W. Urich und R. R. Monle.

7. Atractus favae (Fil.).

B o ulenger, rat. II pag. 31:3.

8:313 a. Erw. (Rüippell, ('at. III C' 5 a als Culumara). 'Tropisches Mmerika. Alte Sammlung $18+5$.

Gemes LXXY'TI. Geophis Wgl.

1. Geophis semidoliatus (D. B.).

Boulenger, ('at. II pag. 316

8316,1 a. Erw. Mexiko. Gesch. 1846 von W. Rössing, hier.

8:316,1 b. Erw. Jalapa, Mexiko. Get. 1893 rom British Iuseum (X. H.). London.

\section{Gemus LXXTIII. Rhabdophidimm Blgr.}

1. Rliabroplidium forsteni (D. B.).

B o ulenger. Cat. II pag. 328.

8:328a. J. 3 ․ Rurukan, Celebes. Erl. 1894 von der Rïppellreise Prof. Dr. W. Kükenthals, Jena.

in einigen untergeortneten Punkten ron dem durch Bonlenger beschriebenen einzigen $q$ dieser Art. Iie Praefrontalen sind etwas lïnger als breit, das Frontale deutlich kürzer als sein Ahstand von der schnauzenspitze; anf der rechten Seite zeigt sich die Andeutung eines kleinen Praeocnlare. - Uben braungran, jede Schuppe mit breitem dunkelbrannem Rande: eine spinalreihe schwarzhrauner Punktflecken, die ladurch entsteht, dab die Schuppen der mittelsten Rückenreihe in ihrel Hinterln̈lfte dunkel gefürbt sind; Kopfoberseite bis zn dem undeutlichen, noch dunkleren Halsbande schwärzlichbrann. Innterseite einfarbig gelbbräunlich, die Hinterränder der sclilder kaum etwas dumkler. - Maße: Totallänge des o 330, schwanzlänge $43 \mathrm{~mm}$. 


\section{Gemus LXXIX. Pseudorhabdium Jan.}

1. Psendorhabdium longiceps (Cant.).

B oulenger, Cat. II pag. 329.

8329, a. Erw. Pontianak, West-Borneo. Gesch. 1893 von Baron Albert ron Reinach, lier.

\section{Genus LIXI. Calamorhabdium noi.}

Terwandt der Gattung Macroculames fithr.. aher mit 13 Maxillarzälmen. obne Praeoculare nnd ohne rortere Temporalen. Abweichend von Rihubdophidium Blgr. und Pseudorhahdium .Jan durch Fehlen der Internasalen, ron Calamariu L. durch die Stelhung des Nasenlochs. die größcre Zahl der Jaxillarzähne um die Zahl der schuppenreihen. - 13 nahezu gleichgroße Oberkieferzälne; I'nterkieferzähne ebenfalls ron ziemlich glejcher (iröße. Kopf nicht vom Halse abgesetzt: Auge klein. mit rumder Pupille. Nasenloch zwischen dem Sasale nud dem ersten Supralahiale eingestochen. Interuasalen. Frenale, Praeoculare und Temporalen fehlen. Körper walzenförmig. Schuppen glatt. ohne Endgrübchen, in 15 Reilen: Bauchschilder germndet. Schwanz kurz. mit stumpfer spitze; schwanzschilder zweireihig. - Hierher als einzige drt:

\section{Calamorlubolinm kuekenthali n. sp.*)}

8:330a. 2 erw. Insel Batjan. Erh. 1895 von der Rïppelheise Prof, Dr. W'. Kükenthals. Jena.

*) Kopf klein. Schmanze zugespitzt. Rostrale fast doppelt so hoch wie breit, auf der Oherseite der Schnauze sichtbar, die Nasalen ron einander tremnend und durch diese an len seiten hogenförmig etwas ansgerandet. Frontale lïnger als breit. so lang wie sein Abstand ron der ichnanzenspitze. kiirzer als die Parietalen. Internasalen fehlen: kein Praeocnlare: ein kleines Postoculare. Temporalen fehlen. ¿ supralabialen, ron denen das dritte und vierte in den Angenkreis treten. Mentale in Berührung mit den vorderen Kimnschildern, die länger sind als die hinteren und mit je 3 Infralabialen in Beriihrung stehen. Hintere Kinnschilder rorn in ler Mitte in Kontakt mit einander.

$$
\begin{aligned}
& \text { Sc buppenformel: Z Sipu. 15; (i. 3. V. } 125 \text {. A. 1. Sc. }{ }^{77}+17+1 \text {. } \\
& \text { ㅇ. } 15 ; . .4 .140 . .1 .,{ }^{10}, 10+1 \text {. }
\end{aligned}
$$

Uben schwarzgrau, lebhaft irisierend, die seitlichen Schuppen mit hellem, weißlichem Mittelflecken; Lippen weißlich: hinter den Parietalen ein breites. aber undeutlich begrenztes. in der Nackenmitte unterhrochenes weißliches Halsband. Ventralen und snbeandalen weißlich mit schwarzen Hinterrändern nnd in unregelmäBigen Längsreihen stehenden schwïrzlichen W'irrfeltleckchen. Mittellinie der schwanzunterseite schwarz.

II B e: ơ Totallänge 184 . Schwanzlänge $17 \mathrm{~mm}$.

$$
\text { q } \Rightarrow 205 \text {. } \quad 10 \%
$$

Fundort: Insel Batjan (Molukken), zwei an der Schnanzenspitze etwas verstoßene stiicke. 


\section{Gemus LXXXI. Calamaria Boie.}

1. Calamaria lumbricoidea Boie.

Boulenger, Cat. II pag. 333 .

833:3 a. Erw. Buitenzorg. West-Jara. Gesch. 1890 von Dr. Arlolf strubell. Bomin.

8333 b. Frw. ठ. Tulkan Tjisurnpan. West-Java. Gek. 1893 ron Hans Fruhstorfer, Berlin.

2. Calamaria vermiformis D. B.

Boulenger, ('at. II pag. 333.

8334 a. Erw. (var. alkeni Bleek.). Sultanat Deli, Nordost-Sumatra. Gesch. 1892 ron Dr. med. O. Benecke, rlaselbst.

8334 b. Erw. (var. dimidiata Bleek.). Vulkan Tjisurupan. West-Java. Gek. 1893 von Hans Fruhstorfer, Berlin.

8334c. Erw. (var. sumatrana Lilth de Jende). Deli. Nordost-Sumatra. Gesch. 1891 von Fritz Beyschlag. daselbst.

3. Calamaria gervaisi D. B.

Boulenger, Cat. II pag. 338.

8338.2 a. Erw.. 3 halbw., 1 jung. Mittel-Lızon. Gesch. 1890 von Konsul Dr. O. Fr. von Moellendorff, Manila.

8338.2 b. Jung. Insel Cehu, Philippinen. Gesch. 1890 von Otto Koch. Ilaselbst.

4. Calamaria simatrana Edel.

Boulenger. Cat. II pag. 339.

8339a. Erw. Sultanat Deli. Nordost-Sumatra. Gesch. 1891 ron Fritz Beyschlag, daselbst.

\section{Calamaria virgulata Boie.}

B onlenger, (at. II pag. 340: B oettger in Semons Zool. Forsch. Hft. 5, 1894 pag. 125 (morlesta var. bogorensis).

8340.1a. Erw. $q$ (var. A). Gunung Pengalengan. West-Java. Gek. 1895 ron Hans Fruhstorfer, Berlin.

8340.1 b. Erw. o (var. ('). Ebendaher. Fek. 1895 von demselben.

8340,1 c. Erw. \& (var. monochrous Bleek.). Eluendaher. Gek. 1895 rom demselben.

8340,1 d. Erw. \& und $q$ (var. ('). Vulkan Tjisurupan, West-Java. Gek. 1893 ron demselben.

8340.1 e. 2 erw. (var. C). Tjiborlas, West-Javit. Gesch. 1894 von Prof. Dr. R. W. Semon. Jena. 


\section{Calamaria collaris Blgr.}

Boulenger', Proc. Zonl, Soc. Londun 1897 pag. 225, Taf. 1t, Fig. 4. 8341 a. Erw. Rurukan in $4000^{\prime}$. Nord-Celebes. Erh. 1895 von der Rïppellreise Prof. Dr. IV. Kïikenthals. Tena.

\section{Calamaria agamensis Bleek.}

Boulenger. Cat. If pag. 343 .

8344 a. Erw, Java. (iet. 1891 vom Zoolog. Institnt der Techn. Hochschule. Karlspuhe.

\section{Calamaria leucocephala J). B.}

Boulenger, ('at. Ifl pag. 34t; Boettger, Ber. offenbach. Ver. f. Naturk. 1873 pag. 38 , Taf. 1 (iris).

8344,1 a. Halbw. (schlecht gehalten. ganz schwarz), Sultanat Deli, NordostSumatra. Gesch. 1891 von Fritz Berschlag, daselbst.

\section{9. (Calamaria semianuulata n. sp.**)}

83łら̃ a. Erw. Borıeo. Gesch. 1886 von Prof. Dr, Oskar Boettger, hier.

*) Ausgezeichnet nicht bloß ilurch anffallende Färbung, sondern anch durch das Rostrale, das - abweichend ron allen beschriebenen Arten der Gattung - dentlich tiefer ist als an seiner Basis hreit. Am nächsten wahrscheinlich der C. brookiei Blgr., the sich aber durch antere Verhältnisse des Rostrale und Frontale, das grïbere Auge nut die sehr abweichente Färbung und Zeichnung unterscheilet. - Hostrale tiefer als breit. von oben gut sichtbar, sein Alistand ron der Schnanzenspitze halb so lang wie die Praefrontalnalt: Frontale so lang wie breit, etwa dreimal so breit wie das supraoculare. viel kïrzer als rie Parietalen; ein Prae- unc ein Postocnlare: Angendurchmesser viel kleiner als sein Abstand ron der Maulspalte. Fïnf Supralahialen. wovon das dritte und das vierte das Auge berïhren; das erste l'aar Infralabialen hinter dem symphysale in Beriihrung mit einander: 2 Paar Kinnschilıler, die hinteren in ihrer Vorderlälfte in Kontakt mit einander. Scluwanz in eine stumpfe spitze ansgezogen.

Schnp penforme l: sin. 13: G. 3, V. 140, A. 1. Sce. ${ }^{27} / 27+1$.

Oberseits rötlichgelh mit 26 innkelbraunen (2nerbinden orler breiten Querflecken. die otwa so breit simd wie die hellen Zwischenrimme; ler dnnkelloraune Nackenflecken am hreitesten: lie schuppen der äuBersten

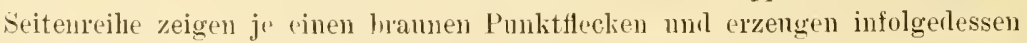
bis zum After einen sehr markierten, nnmter rochenen Punktstreifen. Die Lopfoherseite ist brämlich: ein schief rom Ange nach hinten nud unten zichender. etwas rerschwommener Streifen mir ein zweiter, ihm paralleler, hinter ler Rachenkommissur mach hinten mul miten verlaufender, ïhnlicher. 
10. Calamaria linnei Boie.

Bo ulenger. Cat. II pag. 345 .

8346 a. 2 halbw. (var, rhomboidea Jan). Gunung Pengalengan, West-Java. (iek. 189:) ron Hans Fruhstorfer. Berlin.

8346 b. 3 elw. (var. transversalis .Tan). Bnitenzorg, West-Jara. Gesch. 18:90 ron Dr. Arolf Strubell. Bomm.

8:346 c. Erw. 우 (dieselbe Tarietät). Vnlkan Tjisurupan, West-Jara. Gek. 1893 ron Hans Fruhstorfer.

8346 d. Erw. n. 2 .Junge (var. tessellatu Jan), Buiteuzorg, West.Jara. Gesch. 1890 ron Dr. Adolf Strubell.

8346 e. Erw. o und $q$ (var. bitineate Fitz.). Tulkan Tjisurupan, West-Java. Gek. 1893 von Hans Fruhstorfer.

8346 f. 3 erw. (var. versicolor Ranz.). Tenggergebirge in $1200 \mathrm{~m}$, Ost-Java. Gek. 1891 vou demselben.

8346 g. Erw. (rar. tessellata Jan). Tulkan Tjismupan, Went-Java. Gek. 1893 von demselben.

8346 h. Erw. (rar. contaminata Jan). Eheudaher. Gek. 1893 ron demselben. $83+6$ i. 3 erw. (rar. bilineata Fitz.). Buitenzorg, West-Java. Gesch. 1890 ron Dr. Arlolf Strubell.

11. Calamaria borneensis Bleek.

B o ulenger, Cat. II pag. 347, Taf. 19, Fig. 1.

8347 a. Erw. Baramfluß in Nord-Borueo. Erh. 1895 ron der Rüppelleeise Prof. Dr. Wr. Kükenthals, Jena.

12. Calamaria parimentata D. B.

B o u le nger, Cat. II pag. 348.

8348,1 a. Halbw. Kanton, Süd-China. Gesch. 188j ron Konsul Dr. O. Fr. ron Moellendorff, Janila.

\section{Calamaria lowi Blgr.}

B oulenger, Cat. II pag. 350, Taf. 19, Fig. 4.

8:350 a. Erw. Baramfuß, Nord-Borneo. Erh. 1895 ron der Rüppellreise Prof. Dr. W. Kükenthals, Jena.

aber längerer und breiterer streifen dunkelbrann. Kürper- und Schwanzunterseite einfarbig hell rötlichgelb.

Y a B e: Totallänge 200. Schwanzlänge $25 \mathrm{~mm}$.

Fundort: Bormeo, leider ohne jede nähere Angabe, ein gut gehaltenes Stïck. 
14. Calamaria acutirostris Blgr.

B oulenger, Ann. Mag. X. H. (6) Bd. 17. 1896 pag. 394.

$833 ̄ 1$ a. Erw. Bua Praeng, Süd-Celeles. Gek. 1897 von Hans Fruhstorfer, Berlin.

15. Calamaria gracilis Blgr.

B oulenger, Ann. Mag. N. H. (6) Bd. 18, 1896 pag. 63.

835̄1,1a. Erw. Bua Praeng. Siid-Celebes. Gek. 1897 von Hans Fruhstorfer. Berlin.

16. Calamaria muelleri Blgr.*)

B o u le nge er. Ann. Mag. X. H. (6) Bd. 17, 1896 pag. 394.

8351.2a. Erw. of und ․ Bua Praeng, Süd-Celehes. Gek. 1897 von Hans Fruhstorfer, Berlin.

\section{Subfrmilie :3. Rharhiodontinue.}

Gemus I. Dasypeltis Wgl.

1. Lasypeltis scabla (L.).

Boulenger. Cat. II pag. 35t: Boettger. Ber. Offenbach. Ver. f. Naturk. 1885 pag. 14 (fasciolutu). Ber. Senck. Nat. Ges. 1887 pag. 163 und 188 pag. 75 (typ.), 1888 pag. 76 (var. fasciate) und pag. it (var. petmarum)

83õła. Erw. Akkra, Goldküste. Gesch. 1887 ron Major Dr. L. ron Heyden. hier.

8354 b. Erw. Kinshassa am Stanley Poul, Unter-Cougo. Gek. 1887 von Paul Hesse. Venedig.

var. medicii Bianc. (fasciata Smith).

B o u lenger, l. c. pag. 3506 .

8355 a. 7 Ste. Banana, Luter-Congo. Gek. 1886 von Panl Hesse, Venerlig. 8355 b. 2 erw. Poro Netonna bei Banana, Luter-Congo. Gek. 1887 von Paul Hesse, Venedig.

*) Nach sieben von mir untersuchten Stiicken aus Bua Praeng variiert die Schuppenformel beim

$$
\begin{aligned}
& \text { o Squ. } 13 \text {; G. 3, V. } 141-145 \text {, A. } 1 \text {, sc. }{ }^{17 / 17}-{ }^{18} / 18+1 \text {. } \\
& \text { q } " 13 ; n 3, \text { n } 159-166, \text { ๓ } 1, \quad{ }^{11 / 11}-14 / 14+1 \text {. }
\end{aligned}
$$


val. palmaln ueach.

B onl e 11 ger. l. c. pag. 356 .

8356 a. Erw. u. halbw. Banana, Unter-Congo. Gek. 188う ron Paul Hesse, Venedig.

\section{Subfamilie 4. Homatopsinae.}

\section{Genus I. Hypsirkina Wyl.}

1. Hypsirhina alternans (Rss.).

Bo ul eng e r . Cat. Snak. Brit. Mus. Bd. III, 1896 pag. 4; Re u B. Mus. Senck. Bd. 1, 1834 pag. 155, Taf. !). Fig. 3 (Brachyorrhos); J a n. Icon. Oph. Lief, 30, Taf, 6, Fig. 2; Bo et tger. Ber. Offenbach. Ver. f. Naturk. 1892 pag. 133.

9004,1 a. 4 Junge (Rïppell, Cat. III (' 4 a-e als Eurostus: Originale zu Jans oben citierter Ablildung). Jara. Gesch. 1827 ron Dr. med. Peitsch, Batavia.

\section{Hypsirhina ylumbea (Boie).}

Boulenger, Cat. III pag. 5; Boettger. Ber. Offenbach. Ver. f. Naturk. 1885 pag. 123 und 1888 pag. 83.

9005 a. Halbw. Samarang auf Jara. Gesch. 1876 von Major Dr. L. von Heyden, hier.

9005 b. Jung. Atschin, Nord-Sumatra. Gek. 1882 ron Konrad Kläsi. Ziurich. 9005 c. Jung. Pinang, Malayische Halbinsel. Gesch. 1893 von Dr. Heinrich Lenz. Lïbeck.

9005 d. Erw. Sultanat Deli. Nordost-Smmatra. Gesch. 1894 von Wwe. H. Krï̈mer, hier.

9005 e. 2 erw. Kanton, siil-China. Gesch. 1886 ron Konsul Dr. O. Fr. ron Noellendorff, Manila, und Otto Herz, St.-Petersburg.

9005 f. Erw. Hongkong, Siid-China. Get. 1889 ron Bernhard Schmacker. Shanghai.

9005 g. Halbw. Nyen-hong-li bei Hongkong. Gesch. 1880 von Hans Simon, Stuttgart.

900ذ̉ h. Erw. Insel Hainan. Gek. 1886 von Otto Herz.

9005 i. Elw. (gestopft). Ostindien. Gek. 1846 von Boissoneau.

3. Hypsirhina enhydris (Schnd.).

B oulenger, Cat. 1 II pag. 6.

9007 a. Halbw. Siam. Gesch. 1885 von Konsul Dr. O. Fr. von Moellendorff, Janila. 
9007 1. Halbw. (var. bilineata Gray). Herbert River, Queensland. Get. 1893 rom British Iusenm ( $\mathrm{N} . \mathrm{H}$.), London.

9007 c. 2 erw. (var. bilinente Gray). Java. Gesch, 1880 ron Oberlehrer J. Blum, hier.

4. Hypsirhina bennetti Gray.

Bonlenger, Cat. III pag. 8 .

9008 a. Erw. Insel Hainan, Süd-China, Gek. 1886 von Otto Herz, St.-Peter'sburg.

5. Hypsirhina chinensis Gray.

Bonlenger: ('at. III pag. 8. Taf. 1, Fig. 2: Bocttger. Ber. Offenhach. Ver. f. Naturk. 1885 pag. 123.

9008,1a. Erw. Insel Hainan, Sïd-( hina. Gek. 188(; ron ()t to Herz. St.-Petersburg.

9008.1 b. Halbw. Ehendaher. Gesch. 1894 ron Bernh. schmarker. Shanghai. 9008.1c. 4 erw., 2 jung. Kanton, Süd-China. Gesch. 1885 von Konsul Dr. 0. Fr. von Moellendorff, Manila, und Otto Herz, St.-Petersburg.

\section{Genus II. Homalopsis Kuhl.}

1. Homalopsis buceata (L.).

Boulenger. Cat. III pag. 14.

9014 a. Halbw. Surabaya, Ost.Jara. Gescl. 1889 von Dr. med. Stratz, Haag 9014 l). Halbr. Pinang, Malayjsche Halhinsel. Gesch. 1893 von Dr. Heinr Lenz, Lïbeck.

9014 c. 3 Ste, Buitenzorg. West.Jara. Gesch. 1890 von Dr. Adolf Strubell, Bonn.

9014d. 2 .Tunge. Insel Bangka. Gek. 1881 ron Konrad Kläsi. Zürich.

9014c. Erw. Stabat Estate, Sultanat Deli, Nordost-Sumatra. Gesch. 1893 von Fritz Besschlag, daselbst.

9014 f. 6 Ste. (Rïppell, Cat. III X 1 a-f). Jara. Gesch. 1836-38 ron Dr. med. Peitsch, Batavia, und Dr. W. von Siebold, Leiden.

\section{Gemus III. Cerberus Cur.}

1. ('erberus rhynchops (Schnrl.).

Boulenger, Cat. III pag. 16.

9016 a. Erw. Ningapore. Gesch. 1891 ron Prof. Dr. 0. Boettger, hier.

9016). Erw. Snltanat Deli, Norilost-Sumatra. Geseh. 18.94 rom Wwe. H. Krämer, hier.

9016 c. Zahlr. Ste. Mittel-Luzon, Philippinen. Gesch. 1888 ron Konsul Dr. 0. Fr. von JIoellendorff, Janila.

9016 d. 4 Ste. Bai ron Manila, Luzon. Gesch. 1891 ron demselben. 
9016 e. 3 erw. Patani auf Halmahera. Erh. 189: von der Riippelheise l'rof. Irr. W. Kitikenthals, Jena.

9016f. Erw. Insel Teruate. Erh. 1895 ron derselben.

9016 g. Erw. Jara. Gesch. 1880 ron Oberlelner J. Blum, hier.

9016 h. Erw. Ehendaler Rüppell, Cat. III X 2 a als Homalopsis schneideri. Gesch. 1838 ron Dr. WT. von sieboli. Leiden.

9016 i. Erw. Madras. Gesch. 1889 von 'Theorlor Kolb, daselbst.

9016 k. Erw. (gestopft). Ostindien. Gek. $18+6$ ron Boissoneau.

9016 1. Erw. Philippinen. Gesch. 1897 ron Konsul Dr. O. Fr. von Joellendorff und Otto Koch. C'ebú.

\section{Gemes IT. Fordonia Gray.}

1. Fordonia leucobalia (Schlg.).

Bonlenger, l'at. IIl pag. 21 .

9021 a. Erw. var. unicolor Gray). Insel Mindanao, Philippinen. Gesch. 1888 ron Konsul Dr. O. Fr, von Mloellendorff, Manila.

9021 b. Erw. (dieselbe Varietät), Pontianak. West-Borneo. Gesch. 1893 ron Dr. Heinrich Lenz. Lïbeck.

\section{Subfumilie 5. Dipsadommpleinere.}

\section{Genus I. Georlipsas Blgr.}

1. Geodiusas infralineata (Gthr.).

Boulenger, Cat. III pag. 32, Taf. 3. Fig. 1.

9032 a. Erw. ․ Moramanga, Ost-Madagaskar. Gescl. 1892 von Dr. Alfrerl Voeltzkow, Berlin.

\section{Genus 11. Itycyphus Gthr.}

1. Itycyphus miniatus (Schlg.).

Boulenger, ('at. III pag. 35; B Bettger: Abb. Senck. Nat. (ies.

Brl. 11, 1877 pag. 13, 1879 pag. 464 und Bd. 12, 1881 pag. 444 (I'litodiyges).

9035̆ a. Erw. Nossibé. Gesch. 1876 von Konsul ('arl Ehenau. Lonconbé.

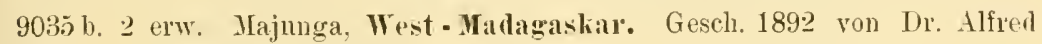
Voeltzkow, Berlin.

9035̃ c. 4 erw. Nossibé. Gesch. 1879-8:3 ron Konsul Carl Ebenau und A. Stumpff, daselbst. 


\section{Genus III. Langaha Brug.}

1. Langaha nasuta shaw.

Boulenger, C'at. III pag. 36: Boettger, Abh. Senck. Nat. Ges. Bil. 11, 1878 pag. 270.1879 pag. 467 und Bil.12. 1881 pag. 447.

9036 a. 4 erw. Nossibé. Gesch. 1881-83 ron Korisul Anton Stumpff, Loucoubé.

2. Laugalia intermedia Blgr.

Boulenger. ('at. III pag. 37; Boettger, Abh. Senck. Nat. Ges.

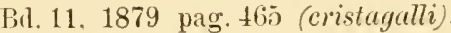

9037.1 a. + erw. Nossibé. Gescl. 1881-8j ron Konsul Anton stumpiff. Loncoubé.

90:37,11. Erw. Ebendaher. (iesch. 1897 von Dr. Alfrerl Toeltzkow. Berlin.

\section{Gemus 11. Eteirodipsas Jan.}

1. Eteirodipsas colubrina (Schlg.).

Boulenger. ('at. III pag. 39; Boettger. Abh. Senck. Nat. Ges. Bil. 12. 1881 pag. 448 (Dipsas) umi Bil. 11, 1877 pag. 16. 1878 pag. 271 und 1879 pag. 467.

9039 a. Jung (var. citrina Bttgr.). Nossihé. Cresch. 1876 ron Konsul Carl Ebenau, Loucoubé.

9039 b. 3 erw. (dieselbe Varietät). Ebendaher. Gesch. 1876 rou demselben. 9039 e. 6 ste. (Typus). Ebendaher. (resch. 1876-85 ron Konsul Carl Ebenau und Anton stumpff. Loucoubé.

9039 d. 2 erw. (daron ein Stiick mit zahlreichen schwärzlichen Punktflecken auf den Ventralen). Majunga, West-Madagaskar. Gesch. 1892 ron Dr. Alfred Voeltzkow. Berlin.

9039 e. 2 erw. Kandani, Siid-Bembatokabai. West-Madagaskar. Gesch. 1892 ron demselben.

\section{Genus IT. Stenoplie Blgr.}

1. Stenophis grannliceps (Bttgr.).

Boulenger, l'at. III pag. 41; Boettger, Abl. senck. Nat. Ges. Bd. 11, 1877 pag. 14. Taf. 1. Fig. 3 und Bil. 12, 1881 pag. 448 (Dipsas gaimardi ral.).

9041 a. 2 erw., 4 jung. Nossibé. Gesch. 1877-83 ron Konsul Anton Stumpff, Loucoubé.

9041 b. Erw. Soalala, West-Madagaskar. Gesclı. 1897 ron Dr. Alfred Voeltzkow, Berlin. 


\section{Genus VI. Tarbophis Fleischm.}

1. Tarbopluis savignyi Blgr.

Boulenger. Cat. III pag. 48; Boettger, Ber. Senck. Nat. Ges. 1878-79 pag. 67 und $1879-80$ pag. 33 (vicax) und 1879-80 pag. 166 (virax f. syriaca).

9047 a. Erw. Jaffa, Syrien. Gesch. 1879 von Major Lr. L. von Heytlen, hier. 9047 b. Kopf. Haiffa, Syrien. Gesch. 1880 ron Hans Simon: Stuttgart.

9047 c. 3 erw. Jerusalem, Syrien. Gesch. 1880 von demselben.

9047 ı. 3 erw.. 1 jung. Sarona bei Jaffa, Syrien. Gesch. 1881 von demselben.

\section{2. 'Tarbophis fallax Fleischm.}

Boulenger, Cat. III pag. 48: Boettger. Zeitschr. f. d. ges. Naturw. Bil. 49. 1877 pag. 287 mil Sitz.- Ber. Berlin. Akarl. 1888 pag. 178 (vivax).

9048 a. Halbw. Burbulitsa bei Volo. Thessalien. Gesch. 1888 von Josef Stussiner, Laibach.

9048 b. Halbw. Nenochóri, Nord-Euböa. Gesch. 1891 von Fr. de Mimont. daselbst.

9048c. Erw. (Rüppell, (at. III WV 1 a als Ailuroplis ciectx). Ost-Europa. Get. 1838 vou Michalielles, Mïnchen.

9048d. Erw. u. 2 Junge (Rïppell, Cat. III IV 1 b-d als A. vivax). Kleinasien. Gesch. 1846 von W. Schneiler, hier.

9048 e. 2 erw. Dalmatien. (iek. 1876 von Banditsch \& ('o.. Triest.

3. 'Tarboulis iberus (Eichw.).

Boulenger. Cat. III pag. 49; Boettger in Radiles Famn. Flor. Casp-Geb. 1886 pag. 72 (vicax).

$90 \pm 9$ a. Erw. IItzchet, Transkankasien. Gek. 1880 von Hans Leder, Janernig (Österr'-Schlesien).

4. Tarboplis semiannulatus (Smith).

B oulenger, Cat. III pag. 51; Boettger. Ber. Senck. Nat. Ges. 1887 pag. 162 (Leptodira).

9051,1 a. Erw. Route / Aus-Keetmanshoop, Grof-Namaland. Gesch. 1887 von Prof. Dr. Hans Schinz, Ziirich.

5. Tarboplis obtusus (Rss.).

Boulenger, Cat. III pag. 52; ReuB, Mus. Senck. Bu. 1, 1834 pag. 137 (Coluber).

905்3 a. Erw. u. jung. (Rüppell, Cat. III V $10 a-b$ als Dipsas aegyptiaca).

Ägypten. Gesch. 1832 von Dr. med. Eduard Rïppell, hier. 
Genus TII. Dipsadomorphus Fiti.

1. Dipsadomorphus trigumatus (Schnd.).

Bonlenger. Cat. Ill pag. 62.

!062 a. Halbw. Madras. Gescll. 1889 ron Theodor Kolb, daselhst.

2. Dipsadomorplus multimaculatus (Boie).

Boulenger. ('at. IlI pag. 63; B o ettger. Ber. Offenbach. Ver. f. Naturk. 1885 pag. 124 (Dipsas).

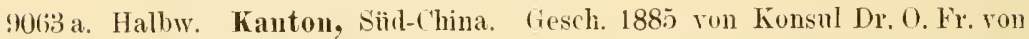
Moellendorff, Manila.

306:3 b. 2 erw. Buitenzorg, Wostodava. Gesch. 1890 von Dr. Adolf strnbeli. Bomm.

9063 c. Erw. (Riuppell, ('at. III V 3 a als Dipses*). Javia. Get. 1838 rom liijksmnsenm in Leilen.

3. Dipsadomorphus ceylonensis Gthr.

Boulenger, Cat. III pag. 66.

9066 a. 2 erw. Ceylon. Gesch. 1846 von Gebr. H. mil W. Worms, hier.

4. Dipsadomoruhus fuscus (Gray).

Boulenger. ('at. III pag. 67; Boettger in Semons Zool. Forsch. Hft. 5. 1894 pag. 118 (Dipsas).

9067 a. Jung. Burnett River, Queensland. Gescl. 1894 ron Prof. Dr. R. W. Semon. Jena.

5. Dipsadomorphus dendrophilus (Boie).

B oulenger, Cat. III pag. 70.

9070 a. Erw. Java (Rïppell. Cat. III V 1 a als Dipsas). Gesch. 1838 ron Dr. W. von Siebold, Leiden.

9070 b. Erw. Elendaher (Rüppell. ('at. III V 1 b als Dipsas). Gesel. 184? von demselben.

var. melanotus Bleek.

Bonlenger. l. c. pag. 71 .

9071 a. Erw. Sultanat Deli, Nordost-Sumatra. Gescl. 1891 ron Dr. met. 0 . Benecke, daselbst.

9071 b. Erw. Stabat Estate, Deli, Nordost-Sumatra. Gesch. 1893 ron Fritz Beyschlag, daselbst. 
var. multicincta Blgr.

Boulenger, 1. e. pag. 71 .

9071.3a. Eir. Dingalan, Prov. Nueva Ecija, Luzon. Geseh. 1893 ron Konsul Dr. O. Fr. ron Moellendorff. Manila.

9071.3 h. Erw. ठ. Sultanat Deli, Nordost-Sumatra. Geseh. 1897 rom I)r. metl. ('. Flach, Aschaffenburg.

var. latifasciata Blgr.

B o u lenger, l. c. pag. 71 .

9071.ja. Erw. Mindanan. Gesch. 1890 von Konsul Pr. (). Fr. von Moellendorff, Manila.

9071.51. Erw. Insel Samar, Philippinen. Geseh. 1897 von demselhen.

6. Dipsadomorphus drapiezi (Boie).

Bonlenger, l'at. IlI pag. 74.

907ta. Erw. (Rïppell. ('at, 1II V 8a als Dipsus). Java. Gesels. 1836 rom Dr. IV. von siebolı, Leiden.

7. Dipsadomorphus augulatus (Pts.).

Bonlenger, Cat. 11 i pag. 75.

9 75 a. Erw. Catanduanes, Philippinen. Gesch. 1891 von Konsul Dr. O. Fr. von Moellendorff. Manila.

\section{Dipsadomorphus irregularis (Merr.).}

Boulenger, ('at. III pag. 75; Boettger, Ber'. offenbach. Ver. f. Naturk, 188j pag. 153 (Dipsas).

9076 a. Erw. Amboina (Riippell, Cat. III V 2 b als Jipsas). Alte Sammlung 1845 .

9076 b. Erw. u. halbw. Insel Batjan. Erh. 1895 ron lex Rïppelheise Prof. Dr. W. Kïkenthals, Jena.

9076 c. Erז. Faro. Salomonsinselu. (iet. 1887 rom British Mnseum (N. H.). London.

9076 d. 2 erw. Ema auf Leitimor, Siid-1mhoina. Gesch. 1890 ron Dr. Alolf Strubell, Bomn.

9076 e. Erw. Insel Duke of York, Nenhritannien. Gek. 1887 von C'. A. Pöhl. Hamburg.

907 fif. Erw. Amboina (Riippell, Cat. III V 22a als Dipsas). Get. 1836 rom Rijksmuseum. Leiden.

907(ig. 2 erw., 2 jung. Insel Ternate. Erlt. 1895 vou der Rüppelheise Prof. Dr. Wr. Kükenthals.

9076 1. Erw. n. jung. Minahassa. Celebes. Erh. 1895 von derselben. 9076 i. (; ste. Soah Konorah anf Hahmahera. Erh. 1895 von dersellen. 


\section{Dipsadomorphus blandingi (Hallow.).}

Bonlenger. Cat. III pag. 77; Boettger, Ber. Senck. Nat. Ges. 1888 pag. it (Dipsas).

9077.2 a. Erw. Poro Nemlao bei Banana. Unter - Congo. Gek. 1886 ron Panl Hesse. Tenedig.

10. Dipsadomorphus cynodon (Boie).

Boulenger, ('at. III pag. 78: Boettger', Abh. Zool. Mus. Drestlen 1894-95, No. 7. pag. 4 (Dipsas).

9078 a. Erw. o (rar. A). Cnlion. Calamianes. Philippinen. Gesch. 1893 ron Konsul Dr, O. Fr. von Moellendorff. Manila.

9078 b. Erw. (var. ('). Majayjay hei Manila. Luzon. (iesch. 1890 ron denselben.

9078c. Erw. (rar. A). Insel Bangka. Gesch. 1886 ron .Mijningenieur II. Koperberg. l'ontianak (Borueo).

\section{Genus IIII. Himantodes D. B.}

1. Himantodes cenchoa (L.).

Boulenger. ('at. III pag. 84.

9084 a. Erw. (Trpus). Mapiri, NebenftnB des eberen Beni, Bolivia. Gesch. 1888 ron Ferdinand Emmel. Arequipa.

$908+$ b. Jung (Typus). Sorata. Bolivia. Gesch. 1891 ron Dr. Heimrich Lenz, Lïbeck.

9084c. Jung (Typus). Trinidad. Gesch. 1895 von F. W. Trich und R. R. Mole, Port of spaill.

9(184il. Erw. (var. lencomelas ('ope). ('aucathal in 500 m. I. S. Columhia. Gek. 1897 von Prof. Dr. Fritz Regel. .Tena.

2. Himantodes elegans. Tan.

B o ulenger. Cat. III pag. 8.ं.

$908 j$ a. Erw. u. jung. Retalhnleu, (4uatemala. Gesch. 1894 ron Carl Fleischmann, daselbst.

\section{Gemus IX. Leptodira Gthr.}

1. Leptortira hotamboeia (Laur.).

Bonlenger. Cat. IIl pag. 89; Boettger, Abh. Senck. Nat. Ges. Bd. 12, 1881 pag. 398 (Crotaphopeltis rufescens) und Ber. Senck. Nat. (ies. 1887 pag. 16:2 und 1888 pag. 72 (rufescens).

yosy a. 2 .Tunge. Boma. Unter-Congo. Ciek. 1886 ron Panl Hesse. Venedig. g08.9 b. '2 halbw. Akkra, (koldküste. Gesch. 1887 ron Major Dr. L. rou Heyden. hier. 
9089 c. Jung. Kapland. Gesch. 1892 ron Oberlehrer .T. Blum, hier.

9089 d. 2 erw. Banana, Unter-Congo. Gek. 1887 von Paul Hesse, Venerlig. y089 e. Erw. (einen Bufo regularis Rss. verschlingend). Povo Nemlao bei Banana. Unter-Congo. Gek. 1886 von demselben.

3089 f. Elw. Siid-Afrika. Gesch. 1873 von Dr. Löwenthal, hier,

9089 g. Halbw. Insel Sansibar. (iesch. 1897 von Dr. Alfred Voeltzliow. Berlin. 9089 h. Halbw. Peccetoni, Wituland, Ost-Afrika. Fesch 1897 von demselben.

\section{Leptodira personata ('ope.}

Ponlenger, Cat. III pag. 93.

909:3,1 a. Erw. 'Tropisches Mexiko. Gek. 1846 von H. Finckh, Ludwigshurg. 9093,1 b. 3 .Innge. Mexiko. Get. 1895 rom British Mnseum (X. H.). London. 9093.1 Erw. 9. Mexiko. Gek. 1887 von C. A. Pöhl. Hambnrg.

\section{Leptodira albofusca (Lacép.).}

Bonlenger. l'at. HI pag. 45.

909.) a. Erw. ('aricas, Yenezuela. (iek. 1877 ron Hübner \& schlesinger. Leipzig.

9095 b Erw. u. halbw. Cairo bei Limon, Ostseite ron Costa Rica. Gesch. 1892-93 vou Carl Fleischmann, Guatemala.

9095 c. Erw. u. halbw. Sïdkiiste ron Triuidad. Gesch. 1888 ron Ingenieur G. Gerold und 1893 ron F. W. Trich und R. R. Mole, Port of Spain. 9075d. Erw. Yucatall. Lebend mit Blauholz hierher gekommen. Gesch. 1891 von Beyerbach jun., hier.

9095 e. 2 erw. Retalhuleu. Guatemala. Gesch. 1894 ron ('arl Fleischmamn. Guatemala.

9095 f. Erw. (Rïppell, ('at. III Y 13 a als Dipsas). T'ropisches Mexiko. Alte Sammlung 1845 .

M95 g. Erw. ㅇ (Riippell. ('at. III V (ie als 1). (mmulatu). Smrinam. Gesch. 1838 ron Dr. med. sichel. hier.

9095 h. 2 erw. Pacasmayo, Nord-Peru. Gesch. 1889) von Max Bamberger. hier. 9095 i. 4 erw.. 2 jung. Carícas, Veneznela. Gek. 1897 von Ingenieur Miiller. hier.

4. Leptodira annulata (L.).

Boulenger, Cat. III pag. 97.

9097 a. Halbw. Puerto Cabello, Venezuela. Gesch. 1894 von Konsul F. Maub, daselhst.

9097 b. Erw. Mapiri, Nebentub des oberen Beni. Bolivia. Gesch. 1888 ron Ferdinand Emmel. Arequipa.

9097 c. 2 erw.. 1 jung (liïppell. ('at. 111 V 6 b-d als Dipsas). Suriuam. Alte Sammlung 1845.

9097 d. Erw. (Ritippell, ('at. III Y 6 a als Dipsus). Eloendalıer. Get. 1838 von Graf Jenison, Heidelberg. 
Genus X. Oxyrrhopus Wgl.

1. Oxyrulupus petolarius (L.).

Bonlenger, Cat. III pag. 101: Re uB. Mus. Senck. Bud. 1. 1834 pag. 148, Taf. 9. Fig. 1 (Coluber digitalis): Ja n. Icon. (1phirt. Lief. 36. Taf. 1, Fig. 1.

9101 a. Erw. (Riippell. Cat. III L 4 a als Iycorlon). Tropisches Mexiko. Gek. $18+6$ ron H. Finckll, Ludwigsburg.

9101 b. Erw. (rar. immaculata D. B.). Mapiri, Nebentuß des oberen Beni. Boliria. Gesch. 1888 von Ferlinanl Emmel. Aręuipa

9101 c. Erw. (dieselbe Varietät). Sorata. Bolivia. Gesch. 1891 von Dr. Heinr. Lenz, Liibeck.

9101 d. Erw. ('ujapa, Staat Matogrosso, Brasilien. Gesch. 1886 ron .Tacob schumacher. hier.

9101 e. 2 erw. (var. B bei Boulenger = digitalis Riss.: Uriginale zu .Jans oben citierter Abbildnng). Jlheos, Brasilien (Riïppell, Cat, 11I L $2 * a-b$ als Lycodon). Gesch. 1836 von Gebr. Koch, hier.

!101 f. Frw. u. jung. Sorata. Bolivia. Gesch. 1891 ron Dr. Heimr. Lenz.

()101 g. 3 Ste. (Riippell. ('at. III L $2 a-b$ als Lycodom). Brasilien. Alte Sammlung $18+5$.

9101 l. Jung (var. digitulis Rss.). Mapiri, Bolivia. Geseh. 1888 ron Ferl. Emmel. 9101 i. Jung (Typus). Medellin, U. S. Columbia. Gek. 1897 ron I'rof. Dr. Fritz Regel, Jena.

9101 k. Erw. (rar. D). ('arácas, Yenezuela. Gek. 1897 von Ing. Mrüller, hier.

2. Oxyruopus rhombifer D. B.

Boulenger. Cat. III pag. 103.

9103 a. Erw. u. jung. Rio (1rande do Sul, Süıl-Brasilien. Gesch. 1873 ron (1)erlehrer Dr. Finger, hier.

91031). Erw. Ebentaher. Get. 1888 ron Prof. Dr. H. von Ihering. dasellsst. 9103 c. Erw. Central-Brasilien. Gesch. 1896 ron Konsul Guillo ron schröter. San José (Costa Rica).

3. Oxyruopus trigeminus 1). B.

Boulenger, Cat III pag 104.

9103,1 a. Halbッ. São Paulo. Brasilien. Gek. 1881 ron Josef Duschanek. hier. 9103,11. Erw. (Riippell. (at. III L 3 a als Lycodon formosus). Brasilien. Get. 1836 rom Naturl. Hofmuseum, Wien.

4. (Oxyruhopus bitorquatus (Gthr.).

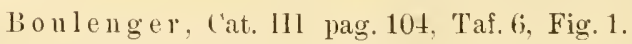

9104 a. Halbw. Sorata, Bolivia. Gesch. 1891 von Dr. Heinr. Lenz, Lübeck. 9104 l. 2 laalbw. Sta. Ana, Prov. ("nzco, Pern. Gesch. 1890 ron Friedrich Emmel. (ielnhansen. 
5. Oxyruopus doliatus D. B.

Bonlenger. C'at. 111 pag. 10t.

9106a. 2 erw. Sta. Ana, Prov. Cuzco, Peru. Gesch. 1890 ron Friedrich Emmel, Gelnhansen.

6. Oxyrhopus fitzingeri (Tschmdi).

Boulenger, ('at. 11 pag. 108.

9108 a. Jung (ohne Kopf). Pacasmayo, Norll-Perı. Gesch. 1887 ron Max Bamberger, hier.

9108 b. Halbw. Peru. Get. 1888 von der Naturhist. Gesellschaft Nürnuerg.

7. Oxyruliopus cloelia (Daud.).

Bo ul enger. C'at. 11 pag. 108.

9) 109 a. Erw. (Riippell, ('at. III II 1 a als (\%oeliu occipitulis). Ilheos, Brasilien. Gesch. 1838 ron Gehr. Koch. hier.

9109 b. Halbw. Săo Paulo. Brasilien. Gesch. 1890 ron Prof. Dr. (O. Boettger, hier.

9109 c. Halbw. (var. occipitalis Jan). Brasilien. Get. 1893 vom Naturhist. Museum in Augsburg.

9L09 d. Elw. (Rïppell, ('at. III O 10 a als Coluber plumbeus). Brasilien. Get. 1840 rom Naturh. Hofmuseum, Wien.

G[09 e. Erw. (gestopft). Brasilirn (Riippell, ('at. III O 10 h als C. plumbeus). Gesch. 1840 von Baron A. von Rothschild dureh Prof. Freyreiß.

$9109 \mathrm{f}$. Kopf und schwanz. ('aparo auf Trinidal. Gesch. 1894 rol F. W. Urich und R. R. Mole. Port of Spain.

8. Oxymopus maculatus Blgr.

Ian, Elenco sist. Ofidi pag. 92. (Bruchyrhytum plumbeum rar.): Boulenger, Cat. III pag. 110. Taf. 6. Fig. ?.

9110 a. Erw. Buenos Aires, Argentina (Tiüpell, Cat. III () 8 a als Callopeltis sp.). Gesch. 1842 von W. A. Bermus, hier.

9. Oxyruopus rusticus Cope.

Boulenger. Cat III pag. 111.

9111 a. Erw. Buenos Mires. Get. 1895 vom British Museum (N. H.), London. 9111 b. Halbw. Start Rio Grande do Sul, Sïl-Brasiliell. Gek. 1886 ron Prof. Dr. H. von thering, daselbst.

10. Oxyrulopus nellwiedi (D. B.).

Boulenger, Cat. III pag. 112.

9112 a. Elw. Insel Tohago, Westindien. Gesch. 1896 von Albrecht Seitz. Hamburg. 
9112 b. Erw. 'Triuidat. Gesch. 1898 ron F. IT. Trich und Ri. di. Mule. T'ort of Spain.

9112 c. Halbw. Venezucla. Gek. 1877 von Ilamburg.

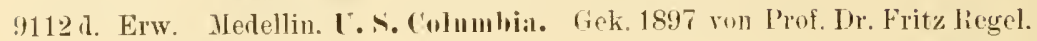
Tena.

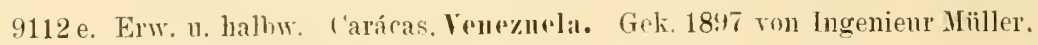
hier.

Gemms IT. Rhinostoma L. R.

1. Rhinostoma guianemse (Trusch.).

Boulenger. ('at. III pag. 114.

9114 a. Halbw. Venezuela. Gek. 1877 von Hambure.

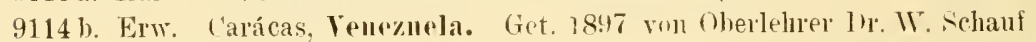
lier.

9114c. Halbw. Baranquilla im Magdalenathal. I. S. Columbia. Gek. 1897 vou Prof. Dr. Fritz Regel. Tena.

9114 1. Erw. u. 2 halbw. ('arácas. Venwurla. Gek. 1897 von Ingenienr Miiller, hìer.

Genus XII. Thammorlynastes Wigl.

1. Tham norlyastes nattereri (Mik.).

Boulenger. ('at. III pag. 116: Boettger. Keitschr. f. l. ges.

Naturw. Bi. 58. 1885 pag. 236.

9116 a. Hałbw. Săo Paulo, Braxilien. Giet. 1881 von .Tosef Duschanek, hier. 9116 h. Elw, Startt Rio Graude do sul. Nïd-Brasilien. Gek, 1886 ron Prof. Dr. H. von llering, rlaselhst.

9116с. Erw. Corrientes, Rep. Argentina. Gesch. 1893 von Dir. Dr. Cárlos Berg, Buenos dires.

9116 r. Erw., 2 halbw. Rio Grande do sul, siiø-IBasilien. Get. 1886-88 von Prof. Dr. H, von thering. Aaselbst.

4116 e. 2 elw. (Riippell, ('at. IIT V 12h-c als Dipsas punctatissima). Ilheos, Brasiliell. Gescl, 1838 von Gebr. Koclı. hier.

2. 'Tham nodynastes punctatissimus (Wgl.).

Boulenger, Cat. III pag. 117.

9117 a. Erw. (Riippell. Cat. III V 12 a als Dipsas). Jlheos, Brasilien. Geseh. 1838 von Gehr. Koch, hier.

\section{Gemus XIII. Tachymenis Wgm.}

1. Taclinmenis peruviana. Wgm.

Boulenger. Cat. III pag. 118.

9118a. Erw. Chile. Get. 188: ron Antsrichter Irüller, Lauterbach (Oberhessen). 
Gemlis XIV. Hemirhagerhis Bttgr.

1. Hemirlugerrlis kelleri Bttgr.

Boulenger. Cat. IIl pag. 119; Boettger, Zool. Anzeiger 1893 pag. 129 .

9119,1 a. Erw. Abdallah, siidlich rom WebiftuBe, Somaliland. Gek. 1893 ron Prof. Dr. Konrad Keller, Ziirich.

Genus XT. Tomodon D. B.

1. Tomodon dorsatus D. B.

Boulenger, Cat. III pag. 121.

\$121 a. 2 erw. Stadt Rio Grande do Sul, Siid-Brasilien. Gek. 1886 von Prof. Dr. H. ron Ihering, dasellst.

Gemus ITI. Amplorhimes Smith.

1. Amplorhinus multimaculatus Smith.

Boulenger, Cat. III pag. 125; Boettger, Ber. Senck. Nat. Ges. 1887 pag. 157 (Psammophylax).

9125,1 a. Erw. Tafelberg hei Kapstadt. Gesch. 1887 von Prof. Dr. Hans Schinz, Zürich.

2. Amplorhinus nototaenia (Gthr.).

Boulenger. l'at. III pag. 125.

$912 j$ a. Erw. Boroma an Sambesi. Fresch. $189+$ von Prof. Dr. O. Boettger. hier.

Gemus IVII. Psendablabes Blgr.

1. Pseudablabes agassizi (Jan).

Boulenger. ('at. II pag. 259 (Contia) und III pag. 126.

9126a a. 2 erw, 1 halbw. Rio Grande do Sul, Niid-Brasilien. Get. 1886-88 ron Prof. Dr. H. ron Ilering: daselhst.

Genus IVIII. Philodryas Wgl.

1. Philodryas aestivus (Schlg:).

Boulenger, Cat. III pag. 128.

9127 a. Iung. Stalt Rio Grande do Sul, Siil-1Brasilien. Gek. 1886 von Prof, Lr. H. ron Ihering, daselbst. 
2. Philodryas viridissimus (1.).

Bonlenger, Cat. III pag. 129.

9128 a. Erw. (Kïppell, Cat. III J' ha als Iterpetmlryes). Surinam. (iesch. 18.36 rom Ir. meil. W. sichel. hicr.

9128 b. Tumg. Sorata. Bolivia. Gesch. 1894 rom Lr. Heinrich Lenz, Liibeck.

\section{Philodryas olfersi (Licht.).}

Boulenger. Cat. III pag. 129: Bnettger. Zeitschrift f. d. ges. Naturw. 13k. 58. 1885 pag. $2: 34$.

9129 a. Erw. (var. lutirostris ('npe). Parawnay. Get. 1886 rom Yaturhist. Institnt Limaea. Berliu.

9129 b. 22 erw. (Typuss. Stadt Rio Grande do sul. Niid-Brasiliell. Gek. 1887 ron Prof. Dr. H. von lhering, laselbst.

9129 c. 2 erw. (var. reimharlti Gthr.). Sorata. Bolivia. Gesch. 1891-94 von Dr. Heinrich Lenz, Liiheck.

9129 il. Erw. (var. latimstris ('ope). Itheos, Brasilien (Riippell. Cat. III P 6 b als Herpetodryas viridissimus). Gesch. 1836 ron Gehr. Koch, hier.

9129 e. Erw. (dieselhe Tarietät) Niil-Amerika. Urek. 1887 ron C. A. Pölıl, Hamburg.

9129 f. Jung (Typus). Corrientes, Rep. Argentina. Ciesch. 1893 von Prof. Dr. Cárlos Berg. Buenos Aires.

9129 g. 2 erw. (Tyms). Brasilien Riipnell. ('at. 1II P 3 a als Herpetorlmus). Get. 1840 ron Graf Jcnison, Heilelhereg.

9129 h. Erw. (Typus). Rio (iraude do sinl, sïd-Brasilien. Get. 1888 ron Prof. Dr. H. ron Thering. dasellost.

9129 i. Erw. (Typus). Ehendaher. Gesch. 1873 von Oherlehrer Dr. Finger; hier.

\section{Philodryas schotti (Schlg.).}

Bonlenger, C'at. III pag. 130; Brettger. Zeitschrift f. d. ges. Naturw. Bd. 58. 1885 pag. 235.

9130 a. 2 erw. Corrientes. Rep. Argentina. Cresch, 1893 ron Direktor Dr. Cárlos Berg, Buenos dires.

9130h. Erw. São Panlo, Brasilien. Gek. 1881 ron .losef Duschanth, hier. !130 c. 2 erw. Central-Brasilien. Gesch. 1886 von Konsul G. von schröter. san José ('usta fica).

91301. Erw, Irmgnay. Get, $188 t$ rom Naturhist. Institut Limnaea, Berlin.

5. Philodryas bolivianus Blgr.

Boulenger, Cat. III pag, 132, Taf. 9. Fig. 1.

9131 a. Erw. Sorata, Bolivia. Gesch. 1891 ron Dr. Heinrich Lenz, Liibeck. 
6. Philodryas elegans ('T'schudi).

Boulenger, ('at. JIl pag. 133: Boettger, Ber. Senck. Nat. Gos. 1889 pag. 31? (Tuchymenis).

9133.1 a. Erw. und 2 Junge. Pacasmayo, Nord-Peru. Gesch, 1889 ron Мax Bamberger, hier.

\section{Genus IIX. Trimerorhinus Smith.}

1. 'T'rimerorhius rombeatus (L.).

Boulenger. Cat. 111 pag. 138; Boet tger, Ber. Offenbach. Ver. f.

Naturk. 1883 pag. 156 (Psammophylar rhombeatus var. trilineata).

9138 a. 3 erw. (Fïppell, ('at. 111 ( $10 \mathrm{a}-\mathrm{c}$ als coronella). Kapland. Alte Sammlung 1845 .

9138b. Erw. (var. trilineate Bttgr.), smithfiehd, Transratal. Gesch. 1882 ron Hans Simon, Stuttgart.

9138c. Halbw. Kaplant. Gek. 188 ron ('. A. Pöhl. Hamburg.

\section{Genus XI. Coelopellis Higl.}

1. Coelopeltis momspessulana (Herm.).

Boulengyer, Cat. III pag. 141: Boettger: Abh. Senck. Nat. Ges.

Bu. 9, 187 t pag. 161 (insignitus), Zeitschr. f. l. ges. Naturw. Bł. 49, 1877 pag. 287. Ber. senck. Nat. Ges. 1879-80 pag. 16\%2 und Abh. Senck. Nat. Ges. 13I. 13. 188:3 pag. 10:3 (lucertina) unl sitz-Ber. Berlin. Akarl. 1888 pag. $17 \%$.

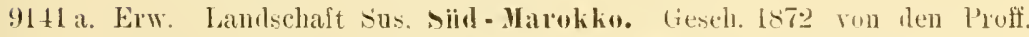
Drt. Freih. ( rou Fritsch. Halle (saale). und , I. Rein. Bomn.

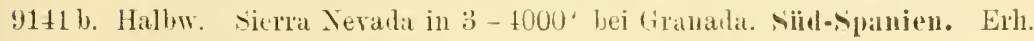
1881 von ter $1 \mathrm{~T}$. Rüppellreise Dr. merl. IT. Kubelt's, schwanheim (Jain .

9141 c. Erw. u, 2 Junge. Sarona bei Jaffa, Syrien. Gesch 1881 ron Hans Simon, stuttgart.

9141 d. Erw. u. jang. Casablanca, Marokko. Gesch. 1881 von demselben.

914 le. Kopf u. jung. Preresa, Epirus. Gesch. 1889 ron César Conéménos. daselbst.

91411. 2 halbw., 3 jung. Casablanca, Iarokko. Gesch. 1881 von Hans simun. Stuttgart.

9141 g. Erw. 'Tnuis. Gesch. 1888 vou Prof. Dr. 1). Boettger, hier.

9141 h. Erw. Tanger, Marokko. Erh. 1891 von der IV. Rüppellreise Dr. merl. II. Kubelt's.

9141 i. Erw. Niit-Enmpa. Gesch. 1886 ron Hans simor. stuttgart.

9141 k. Erw. " 2 Junge. Bisera, Ilgerien. Erh. 1884 ron der V.liiipgellreise durch Lr. med. Wr. Kobelt, schwanheim (Iain). 
91411. Halbw. Xenochóri, Nord-Euböa. Gesch. 1891 rou Fr. de Mimont, daselbst.

9141 m. Jung. Siid-Europa. Gesch. 1862 ron Adam Becker, Sydney.

9141 n. Jung. Burbulitsa bei Tolo, Thessalien. Gesch. 1888 von Josef Stussiner, Laibach.

9141 o. 2 Köpfe. Casablanca, Marokko. Gesch. 1889 rou Dr. med. H. Schädle. Tanger.

9141 p. Jung. Jerusalem. Gesch. 1880 ron Hans Simon, Stuttgart. 9141 q. 2 erw. Sarona bei Jaffa. Syrien. Gesch. 1881 ron demselben.

9141 r. Erw. Landschaft Sus, Süd-Marokko. Gesch. 1872 von den Proff. Drr. C. v. Fritsch und J. J. Reill.

9141 s. Erw. (Rüppell, Cat. III h 1 a als lacertina var.). Siid-Europa. Alte sammlung 18 เร.

2. Coelopeltis moilensis (Reuß).

Boulenger: Cat. III pag. 1ł3; ReuB. Mus. Senck. Bd. 1; 1834 pag. 142, Taf. 7, Fig. 1 (Coluber).

9143a. Erw. Tunesien. Gesch. 1896 von der Neuen Zoolog. Gesellschaft, hier. 9143 b. Erw. Kiistenreise ab Waryla. Algerien. Gesch. 1893 ron Prof. Dr. A. König, Bonn.

9143 c. Erw. (Rüppell, Cat. III R 1 a als lacertina rax.), Arabien. Gesch. 1834 ron Dr. med. Eduard Rïppell, hier.

9143 r. 2 erw. Siul-Tunis. Gesch. 1897 ron rer Neuen Zoolog. Gesellschaft. hier.

\section{Genus XXI. Rhamphiophis P'ts.}

1. Rhamphiophis multimaculatus (Smith).

Boulenger, ('at. 11I pag. 148; Boettger. Ber. Senck. Nat, Ges. 1886 pag. 4 (Dipsina).

9148,1 a. Erw. Route I Ans-Angra Pequena, Grofi-Namaland. Gesch. 1887 von Prof. Dr. Hans Schinz. Zürich.

9148,1 h. Erw. Angra l'equena. (Arofs-Namaland. Riesch. 1886 ron l'rof. De. (I) sliar Schneider. Dresilen.

Gems XIII. Hromophis Pts.

1. Dromophis prateoluatus (Schlg.).

Boulenger, (at. 111 pag. 150).

9156) a. HaJbw. Akkra. (ioldkiistr. Gesch. 1887 ron Major br. L. ron Heylen, hier. 


\section{Gemes XXIII. Taphrometopon brandt.}

1. Taphrometopon lineolatum (Brandt).

boulenger', ('at. III pag. 151.

9151 a. Erw. Transkaspien. Gesch. 1896 von der Xeuen Zoolog. Gesellschaft, lier.

9151 1. Erw. T'schilischljür, 'Transkaspien. Gesch. 1888 von Staatsrat Dr. Gustar voll Radde, Exez. Tiflis.

91う̇ c. Erw. Tsehinas, T'urkestaı. Get. 187!) rom Naturhistor. Iusemm. St.-Petersburg.

ylòl d. Erw. Bacharden, 'Transkaspien. Gesch. 1896 von der Neuen Zoolog. Gesellschaft, hier.

9151 e. Erw. Ebendaher. Gesch. 1896 von derselben.

\section{Genus XIIT. Psammoplis Boie.}

1. Psammophis schuliari (Forsk.).

Boulenger, Cat. IIl pag. 157; RenB, Mus, Senck. Bd. 1. 1834 pag. 139 (Coluber lacrymans); Boettger. Ber. Senck. Nat. Ges. 1879-80 pag. 63 (moniliger var. hierosolymitanu), pag. 164 (moniliger var. punctata) und in Kobelt's lieise Alg. Tunis 1885 pag. 462 (sibilans var. punctatu).

9157 a. Erw. (Typus). Bordj Saarla bei Biscra. Algerisehe Nilhara. Gesch. 1893 ron Prof. Dr. Alex. König. Bonı.

9157 b. Erw. (Typus). Biscra, Mgerien. Gek. 1885 von Lonis Fischer. daselbst. 9157 c. Halbw. (var. C). İgypteu (Rïppell. ('at. 111 O 15 a als Coluber floriceps). Gesch. 1834 ron Dr. met. Eiluard Rïippell, hier.

9157 d. 2 elw. (Typus). Jaffa. Nyrieu. (iesch. 187 ? ron Major Dr. L. von Heyrlen. hier.

9157 e. Kopf u. 2 halbw. (Typus). Ehendaher. Gesch. 1880 von Hans simon, Stuttgart.

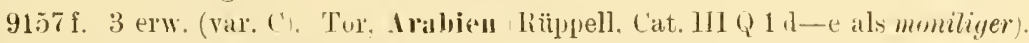
Geseh. 1834 yon 1'r. med. Eduard Rüplell, hier.

$9157 \mathrm{~g} .3$ erw. (Typus). İgypen (Rüppell, Cat. III Q 1 a-c als monitiger). Gesch. 1834 ron Dr. merl. Eduard Riippell, hier.

9157 h. Erw. (Typus). Siil-Tunis. Gesch. 1896 ron der Nenen Zoolog. Gesellschaft. hier.

9157 i. 2 erw. ('Typus u. var. C.). Ebendilher. Gesch. 1897 ron derselhen.

\section{Psammoplis punctulatus I). B.}

Houlenger. C'at. 11I patg. 159; Boettger. \%ool. Anzeig'er 1893 pag. 11!.

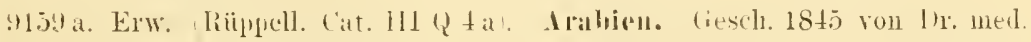
Eduard Rüppell, lier. 
3. Psammophis sibilans (L.).

Boulenger, Cat. III pag. 161; Boettger. Abh. Seuck. Nat. Ges.

Bd. 12, 1881 pag. 395 und Ber. Senck. Nat. Ges. 187i —88 pag. 53.

9162 a. Erw. (var. D). Abessynien. Gek. 1887 ron C. A. Pöhl, Hamburg.

9162 b. Erw. (var. F). West-lfriki. Get. 1875 ron Oberlehrer J. Blum, hier.

9162 c. Erw. (var. F). Poro Netonna bei Banana. Unter-Congo. Gek. 1887 ron Paul Hesse, Venedig.

91602 d. Erw. (Typus). Angra Pequena. (rools- Yamaland. Gesch. 1886 von Prof. Dr. Oskar Schneider. Dresten.

9162 e. Erw. (Typus). Ronte / Aus-Angra Pequena. (Hrols-Samiland. Gesch. 1887 ron Prof. Dr. Hans Schinz, Zürich.

9162 f. Erw. (var. F), Odumase, Ost-(ioldkiiste. Gesch. 1892 ron Hans Simon, Stuttgart.

9162 g. Erw. (rar. F). Banana, Unter-Congo. Gek. 1885 ron Paul Hesse. 9162 h. Halbw. (var, F). Kaplaud? (Rüppell, Cat. III Q 2a als crucifer?). Alte Sammlung 1845 .

9162 i. 2 erw., 1 jung (var. F). Banana, Luter-Congo. Gek. 1886 ron Paul Hesse.

9162 k. 2 erw. Elsendaher. (rek, 1886 ron demselben.

9162 1. 2. erw. (var. E und F). Peccetoni, Wituland, Ost-Afrika. Gesch. 1897 von Dr. Alfred Voeltzkow, Berlin.

$9162 \mathrm{~m}$. Erw. (Typus). Ïgypten. Gesch. 1897 ron ler Neuen Zoolog. Gesellschaft, hier.

4. Psammophis elegans (Shaw).

Bonlenger, Cat. III pagr. 167; Boettger, Abl. Senck. Nat. Cies. Bd. 12, 1881 pac. 395.

916ī a. Erw. Odumase, Ost-Goillkiiste. Gesch. 18!2 ron Hans simon, stuttgart.

9167 b. Erw. Lagos, West-Afrika. Gek. $18 \bar{i}$ run Hanburg.

5. Psallllophis crucifer (Dand.).

Boulenger. ('at. III pag. 169; Buet tger. 22.203. Ber. Offenbacl.

Ver. f. Naturk. 1883 pag. 156 .

9169, a. Erw. Kapland. (iet. 188: ron (bherlehrer J. Blum. hier.

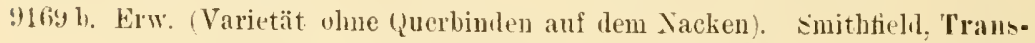
vaal. Gescl. 1882 ron Hans Simon, struttgart.

6. Psammophis angolensis (Boc.).

Boulenger, (at. HIl pag. 170; Boettger, Ber. Senck. Nat. Ges. 1888 pag. ò (Drumophis).

9170a. Erw. Ambrizette. Mmgoliı. Gek. 1887 ron l'aul Hesse, Venedig. 


\section{Genus XXI. Wimophis Gthr.}

1. Mimophis mahfalensis (Grand.).

Boulenger, Cat. III pag. 171; Boettger, Abh. Senck. Nat. Ges.

Bd. 12, 1881 pag. 445 (madagascariensis).

9171 a. 2 erw. Insel Nossibé. Gesch. 1880-85 von Konsul Carl Ebenau und Anton Stumpff, Loucoubé.

9171 b. 2 halbw. Ebendaher. Gesch. 1897 von Dr. Alfred Voeltzkow, Berlin. 9171 c. Halbw. Soalala, West-Matlagaskar. Gesch. 1892 von demselben.

9171d. 4 erw. Majunga, West-Madagaskar. Gesch. 1892 ron demselben. 9171 e. 3 erw. Kandani, Siid-Bembatokabai, West-Madagaskar. Gesch. 1892 voll demselben.

\section{Genms XXII. Psammodynastes Boie.}

1. Psammodynastes pulveruleutus (Boie).

Boulenger, Cat. III pag. 172.

9172 a. Erw. Tulkan Pengalengan, West-Java. Gek. 1895 von Hans Fruhstorfer, Berlin.

9172 h. Erw. Baramfluß in Nord-Borneo. Erh. 1895 ron der Rïppetlreise Prof. Dr. W. Küikenthals, Jena.

9172 e. Erw, West-Jara. Gel. 189;3 ron Hans Fruhstorfer.

9172 d. 2 halbw. Insel Leyte, Philippines. Gesch. 1893 von Konsul Dr. O. Fr. von Moellendorff, Mlanila.

9172 e. 2 Ste. Vittel-Luzwn, Philiptinen. (iesch. 1890 von demselben.

9172f. Erw. Tenggergebirge in 1200 m. 0st.Java. Gek. 1891 von Hans Hruhstorfer.

9172 g. Erw. (Riippell, Cat. II (1 3 a als Psommophis). Jara. Gesch. 1840 von br. II. ron siebull, Leirlen.

917.2 h. 2 lablbw. Insel Hindana, l’hilippinen. Gesch. 1890 yon Konsul Dr. O. Fr, von Bloellendur'ff.

9172i. Frw. Sultanat Deli, Nordost-sumatra. Gesch. 1892 von Dr. med. U. Benecke, rlaselbst.

9172k. Halbw. Ehendaluer. Gesch. 1879) von W. von schouler, Wieshaden. 9172 l. Halbw. Bindjey in Deli. Nordost-sinmatril. Gesch. 1890 von Theorlor und Egbert Engrelharl. laselbst.

977u. Erw. Insel samar, Philippinen. (ieseh. 1897 rou Konsul Ir. O Fr. ron Moellemderff.

2. Psammodyuastes pictus Gthr.

Boulenger, Cat. III pagg. 1 it.

917ta. Erw. Sultanat Deli, Nordost-Sumatra. Gesch. 1894 ron Wwe. H. Liämer, hier.

917łb. Hallw. Lusel Bangka. Gesch. I886i von Prof. D1, Uskar Bocttger, hier. 
Genus XYIII. Macroprotodon Guich.

1. Macroprotodon cucullatus (Geoffr.).

Boulenger, Cat. III pag. 175; Boettger, Abh. Senck. Nat. Ges. Bd. 12,1880 pag. 374 und pag. 387, Bd. 13, 1883 pag. 96 und in Kobelt's Reise Alg. Tunis. 1885 pag. 457 (Coronella).

9175 a. Halbw. Zeuatta (S'tuatta), 8 Stunden von Casablanca. Marokko. Gesch. 1893 ron Dr. med. H. Schädle, Tanger.

9175 b. Erw. Tisi Tacherat, Marokko. Gesch. 1873 vou den Proff. Drr. ('. von Fritsch, Halle (Saale), und J. J. Rein, Bonn.

9175c. 2 elw. Palma anf IIallorka, Balearen. Gek. 1880 von Prem.-Lieut. Dr. Friedr. Will, Erlangen.

9175 d. 2 erw. Algeciras, Süd-Spanien. Gesch. 1881 ron Hans Simon, Stuttgart.

9175 e. Erw. Casablanca, Marokko. Gesch. 1889 von Dr. med. H. Schädle. 9175 f. Erw. u. 4 halbw. Ebendaher. Erh. 1881 von Hans Simon und von der IV. Rüppellreise Dr. med. W. Kobelt's, Schwanheim (Main).

$9175 \mathrm{~g}$. Zahlr, Ste. Tanger, Marokko. Gesch. 1881 von Hans Simon, Stuttgart. 9175 h. 3 erw., 3 jung. Stadt Marokko. Gesch. 1881 von demselben.

9175 i. Erw. Biscra. Pror. Constantine. Algerien. Erh. 1885 von der V. Riippellreise Dr. med. W. Kobelt's. Schwanheim (Main).

\section{Genus XXTIII. Dryophis Dalm.}

1. Dryophis dispar (Gthr.).

Boulenger, Cat. III pag. 179.

9179 a. 4 Ste. Kodaikanal, Palni Hills, Siid-Indien. Gesch. 1889 von Theodor' Kolb, Madras.

2. Dryophis fronticinctus Gthr.

Boulenger, Cat. III pag. 179.

9179.1 a. Erw. Pegn, Hinterindien. Gesch. 1887 ron Prof. Dr. O. Boettger. hier.

3. Dryophis prasinus Boie.

Boulenger, rat. 111 pag. 180.

9181 a. Erw. Minahassa anf Celebes. Erh. 1895 ron der Rüppellreise Prof. Dr. W. Kïkenthals, Jena.

9181 b. \& Ste. Baramfluß in Nord-Borneo. Erh. 1895 von derselben.

9181 c. Halbw. (var. laetı ('ope). Umgebung ron Manila. Lnzon. Gesch. 1891 ron Konsul Dr. O. Fr. Fon Mloellendorff, Manila.

9181 d. Erw. (Riippell, Cat. III U 1 a als Dryinus suctutus). Javi. Gesch. 18:38 ron Dr. med. Peitsch. Bataria.

9181 e. Halbw. Atschin, Nord-sumatra. Gek. 1882 von Konrad Kläsi, Zürich. 
9181 f. Halbw. (var. laeta Cope). Insel Cebú, Philippinen. Gesch, 1890 von Konsul Dr. O. Fr. von Moellendorff.

$9181 \mathrm{~g}$. Erw. Pontianak, West-Borveo. Gesch. 1893 von Baron Albert von heinach, hier.

9181 h. 3 halbw. Sultanat Deli, Nordost-Sumatra. Gesch. 1886 von Clemens Holnwiesner, hier.

9181 i. Erw. Ostindien. Gek. 1887 von C. A. Pöhl, Hamburg.

9181 k. 2 erw. Bindjey in Deli, Nordost-Sumatra. Gesch. 1890 von Theodor und Egbert Engelhard, hier.

9181 l. Halbw. (Rïppell, Cat. III U 2 c als Dryinus). Java. Get. 1838 vom Rijksmuseum in Leiden.

9181 m. 2 Ste. Insel Bangka. Gek. 1881 von Konrad Kläsi, Zürich.

9181 n. 2 Ste. Insel Culion, Calamianes, Philippinen. Gesch. 1893 von Konsul Dr. O. Fr. von Moellendorff.

9181 0. 2 halbw. Buitenzorg, West-Java. Gesch. 1890 von Dr. Adolf Strubell, Bonn.

9181 p. 2 erw. (Rüppell, Cat. III L 2 a-b als Dryinus). Java. Gesch. 1838 von Dr. W. von Siebold, Leiden.

9181 q. Zahlr. Ste. (var. laeta Cope). Mittel-Luzoı. Gesch. 1888 von Konsul Dr. O. Fr. von Moellendorff.

9181 r. Erw. Banjermasin, Südost-Borneo. Gek, 1894 von Wwe. H. Krieb, hier.

9181 s. Erw. Dongala Kabonga, West.Celebes. Erh. 1894 von der Rüppellreise Prof. Dr. W. Kïlienthals, Jena.

4. Dryophis fasciolatus (Fisch.).

Boulenger, Cat. 11 pag. 18:2.

9182 a. Jung. Sultanat Deli, Nordost-Sumatra. Gesch. 1886 von Clemens Hohwiesner, hier.

5. Dryophis mycterizans (L.).

B o u l e $n g \in r^{\circ}$, Cat. 11 l pag. 18:.

918.3 a. 2 erw. Ceylon. Gesch. 1846 ron H. Worms, hier.

\$183 b. Erw. (líiippell, ('at. III U 1 b als Dryinus nasulus). Bengalen. Gesch. 1838 von Dr. med. Peitsch, Bataria.

\$183 c. 4 Ste. Madras. Gesclr. 1889 ron Theodur Kolb, daselbst.

\$183 d. Erw. Nian. (iesch. 1885 ron Konsul Dr. U. Fr. von Hoellendorff, Manila.

Genus XXLI. Thelutornis Smith.

1. Thelotornis kintlandi (Hallow.).

Boulenger, ('at. IIl pag. 185; Boettger, Ber. Senck. Nat. Ges. 1888 pag. 65 (Dryiophis).

\$185̃ a. Erw. Gabnn. Gek. 1877 von Hanburg. 
9185 b. 2 erw. Poro Netonna bei Banana, Unter.Congo. Gek. 1887 von Paul Hesse, Venedig.

9185 c. Erw. \& mit Eiern. Povo Nemlao bei Banana, Cuter-Congo. Gek. 1886 von demselben.

9185 d. Erw. Kamerun. Gek. 1897 ron Lr. Georg Kraatz. Berlin.

\section{Genus XXY. Dispholidus Dutern.}

1. Dispholidus typus (Smith).

Boulenger, Cat. III pag. 187; Boettger, Abh. Senck. Nat. Ges.

Bd. 12, 1881 pag. 397 (Bucephalus capensis).

9187 a. 2 erw. (var, belli Smith). Siid-Afrika. Gesch. 1873 ron Dr. Löwenthal, hier.

9187 b. Erw. (Typus). Ehendaher. Gesch. 1873 ron demselben.

\section{Genus XXXI. Oxyluelis Wgl.}

1. Oxybelis acuminatus (Wied).

Bonlenger. Cat. III pag. 192; B oettger, Ber. Senck. Nat. Ges. 1889 pag. 31t (Dryiophis).

9192 a. Erw. Insel Tobago, Westindien. Gesch. 1894 von Albrecht Seitz, Hainburg.

9192 b. Erw. Retalhuleu, Guatemala. Gesch. 1895 ron Carl Fleischmann, daselbst.

9192 c. Jung. Trinidal. Gesch 1893 von F. W. Urich und R. R. Mole, Port of Spain.

9192 r. Erw. Pacasmayo, Nord-Peru. Gesch. 1889 von Max Bamberger, hier. 9192 e. 2 crw. (liïppell, Cat. III U $3 \mathrm{a}-\mathrm{b}$ als Dryimus aeneus). Itheos, Brasilien. Gesch. 1837 ron Gebr. Koch: hier.

4192 f. Erw. Carácas, Yenezuela. Gek. 1897 von Ingenienr Miiller, hier.

\section{Gerus XXXII. Chrysopelea Boie.}

1. Chrysopelea rhodopleuron Boie.

Boulenger, Cat. III pag. 195.

9195,1 a. Erw. Wai aut Hitr, Norl-Amboina. Gesch. 1890 ron Dr. Adolf Strubell, Bonn.

2. Chrysopelea ornata (Shaw).

Boulenger; Cat. 11I pag. 196: Boettger, Ber'. Offenbach. Ver. f. Naturk. 1888 pag. 84 .

91!tia, 2 halbw. (var. A. Atschin, Mord-siunatra. (iek. 188.2 rom linnranl Kỉäsi, Zürich. 
9196 h. Erw. (rar, A). Stahat Estate in Deli. Nordost-Nimatra. Gesclı. 189:3 rom Frit\% Reyschlas, rlaselbst.

9l96ic. Halbw. (var. B). Barautioß in Nord-Bornon. Erh. 1895 vou der Rïppellreise J'rof. Dr. IT. Kiikenthals, Jena.

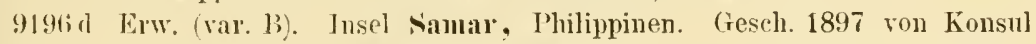
I)r. (). Fr. von Moellenilorff, Manila.

9196 e. ó Ste. (var. B u. H). Mittel-Iuzou. Gesch. 1890 ron demselben. $9196 \mathrm{f}$. 2 ен. (var. I)). Siam. Gesch. 1885 vou remselben.

3. Chryopelea chrysochlora (Schlg.).

Boll le $l$ ger'. ('at, III pag. 198.

9198 a. Erw. Insel Bangka. Gek, 1881 von Komrar Kläsi, Ziurich.

9198 b. Erw. Sultanat Deli, Norlost-Numatra. Giesch. 1886 von Clemens Hohwiesner, hier.

\section{Genus XXXIII. Frythrolamprus Wgl.}

1. Evythrolamprus a esculapii (T.).

Boulenger, Cat. III pag. 200.

9200a. Erw. (var. venustissima Wiel). San José, Costa Rica. Gesch. 1894 von Konsul Guiclo von Schrïter, daselbst.

9200h Erw. (lieselbe Varietät). Elendalıer. Gesch. 1892 von (arl Fleischmann. dasellost.

9200 c. Erw. (rar. agilis L.). Nurimam. Alte Sammlnng 1845.

9200 r. 2 erw. (typ.). Brasilien (Riippell, ('at. II $(\mathrm{i}$ ib-c als renustissimus). Gesch. 1828 von Cordier.

9200 e. Jung (var. tetruzona Jan). Solata. Bolivia. Gesch. 1891 von Dr. Heimr. Lenz, Liibeck.

9200f. Erw. (var. monozona Jan). Illeos, Brasilien (Rüppell, Cat. III G 1 a als venustissimus). Gesch. 1828 von Gebr. Koch, hier.

$9200 \mathrm{~g}$. \& Ste. (var. tetruzona Jan). Japiri, NebenfluB des oberen Beni. Bolivia. Gesch. 1888 von Ferdinand Emmel. Arequipa.

$9200 \mathrm{~h} .2 \mathrm{el} w$. (val. guentheri Garm.). Surimam (Riippell, Cat. III G $2 \mathrm{~h}-\mathrm{c}$ als agilis). Get. 1830 vom Rijksmuseum in Leiden.

9200 i. 3 erw. (var. agilis L.). Snrinan (Riippell, Cat. III G 2 d-f als agitis). Alte Sammlung 1815.

9200 k. Erw. 11. 2 Junge (var. venustissima Wied). Central-Brasilien. Gesch. 1896 von Konsul Guido von Schlöter, San Josẻ (Costa Rica).

92001. Erw. (var. monozona Jan). Medellin, U. S. Columbia. Gek. 1897 von Prof. Dr. Fritz Regel, Jena.

9200 m. Erw. (var. venustissima Wierl). Amalti, U. S. Columbia. Geh. 1897 ron demselben.

9200 n. Erw. (dieselbe Tarietät). (arácas, Venezurla. Gek. 1897 von Ingen. Miiller, hier. 
2. Erythrolamprus fissidens (Gthr.).

Fioulenger, Cat. III pag. 207.

9207 a. 3 erw. Retalhuleu, Guatemala. Gesch. 1895 von Carl Fleischmann. laselbst.

3. Erythrolamprus bipunctatus (Gthr.).

Boulenger, Cat. III pag. 208.

9208 a. Halbw. Nicaragua. Gesch. 1892 von Prof. Dr. O. Boettger, hier.

Genus XXXIV. Homalocranium D. B.

1. Homalocranium melanocephalum (L.).

Boulenger, Cat. III pag. 215.

9215 a. Halbw, Trinidad. Gesch. 1895 von F. W. Trich und R. R. Mole. Port of Spain.

9215̃ b. Halbw. (var. pallida Cope). U. S. Columbia. Gesch. 1894 von Konsul F. C. Lehmann, Popayán.

9215 c. Halbw. Trinidad. Gesch, 1893 von F. W. Uricl und R. R. Mole.

9215 d. Erw. (Rüppell, Cat. III G 9 a als Coronella). Suriuau. Alte Sammlung 1845 .

9215 e. Erw. (var. pallida Cope). Central-Brasilien. Gesch. 1896 von Konsul Guido von Schröter, San José (Costa Rica).

2. Homalocranium semicinctum D. B.

B o ulenger, Cat. III pag. 219.

9219,1 a. Erw. Baranquilla im Magdalenatlual, I. ‘. Columbia. Gek. 1897 von Prof. Dr. Fritz Regel, Jena.

3. Homalocranium schistosum Boct.

Boulenger, Cat. III pag. 221.

9221,1 a. Erw. Matagalpa, Nicaragua. Gesch. 1897 ron Prof. D1. O. Boettger. hier.

4. Homalocranium planiceps (Blainv.).

B o ul enger, Cat. III pag. 226.

9226.1 a. Halbw. Florida. Gesch. 1896 von Dr. merl. A. Zipperlen, Cincinnati (Ohio). 


\section{Gems XXXV. Stenorlima D. l'.}

1. Stenurhina degenhariti (Berth.).

Bowlenger. l'at. III pag. 229.

92:30 a. 2 erw. (rar. Bc). Mexiko. Gek, 1846 von H. Finckb, Lndwigsburg. 9230 h. Erw. (rar, A). ('arácas. Yemezuela. Gek. 1897 von Ingeniemr Müller, hier.

Genns XXXVI. Apostolepis Cope.

1. Apostolepis assimilis (Reinh.).

Boulenger. Cat. III pag. 234 .

9234 a. B elw. Central-Brasilien. Gesch. 1896 von Konsnl Gnilo von Schröter. San José ('osta Rica).

Genus XXXVII. Elapomorphens D. B.

1. Elapomorphus lemniscatus D. B.

Boulenger, Cat. III pag. 242.

9242 a. Erw. u. 2 Junge Rio Grande do Sul, Süd-Brasilien. Get. 1888 ron Prof. Dr. H. von Ihering, dasellost.

9242 b. Erw. Ehendaher. Gesch. 1873 von Oberlehrer Dr. Finger, hier.

tienus XXXVIII. Xenocalamus Gthr.

1. Xenocalamus mechowi Pts.

Boulenger, ('at. III pag. 248; Boettger', Ber. Senck. Nat. Ges. 1888 pag. 47

9248,1 a. Erw. Kinshassa am Stanley Pool, Congo. Gek. 1887 von Panl Hesse, Venedig.

Genus XXXIX. Micrelaps Bttgr.

1. Micrelaps muelleri Bttgr.

Boulenger, Cat III pag. 249; Boettger, Ber. Senck. Nat. Ges. 1879-80 pag. 137. 'Taf. 3, Fig. 2.

9249 a. 3 erw. Jerusaleu. Gesch. 1879-84 von Hans Simon, Atuttgart.

Gemus XL. Aparallactus Smith.

1. Aparallactus werneri Blgr.

Boulenger, Cat. III pag. 25̆7, Taf.11, Fig. 1.

9257 a. Erw. Usambara. Ost-Afrika. Get. 1896 vom Naturh. Yuseum in Berlin. 
Grmes XLI. Elapops Gthr.

1. Elapolis modestus Gthr.

Boulenger, Cat. III pag. 262.

9263 a. Erw. (rof-Popo, West-Afrika. Gesch. 1894 vou Prof. Dr. O. Boettger, hier.

9263 b. Erw. Kamerun. Gesch. 1896 ron Dr. Heinrich Lenz. Liibeck.

9263 c. 3 Ste. El)endalıer. Gek. 1897 ron I)r. Georg Kraatz, Berlin.

\section{Subfamilie (5. Hydrophiinae.}

\section{Genus I. Hydrus Schud.}

1. Hydrus platurus (L.).

Boulenger. Cat. III pag. 267; B o ettger. Ber. Offenbach Ver. f. Naturk. 1892 pag. 88.

9267 a. Jung (Trpus: Monstrosität mit rier Nasalen mul rier Nasenlöcher’u).

Ceylon. Gel. 1889 ron Hans Fruhstorfer. Berlin.

9267 ). Halbw. (Typus. Manila, Lazon. Gesch. 1830 ron Konsul Dr. O. Fr. von Mroellendorff, daselbst.

9267 c. Halbw. (var. ormata Gray). Niulsee. (rek. 1887 ron ('. A. Pölll. Hamburg.

9267 d. Erw. (Typus), Niidsee. Gek. 1879 von demselben.

9267 e. 2 erw. (var. ornatu Gray). Indischer Ocean (Fiippell, ('at. III I 2 a - h

als Hydrophis pelumis). Gesch.1838 ron Dr. WT. ron siebold. Leiden.

$9267 \mathrm{f}$. Hallww. (Typus). Java (Riippell, Cat. III Y 3 a als Hydrophis hicolor). Gesch. 1836 von Dr. merl. Erharil Riippell. hier.

9267 g. Erw. (C'l)ergang rom Typus zur var. maculuta .Tan). C'eylon. Gesch. 1881 von Gustar Herath, hier.

9267 l. Erw. u. jung (Typus). Bai von Manila, Luzon. Gesch. 1891 von Konsul Dr, O. Fr. von Moellentorff.

9267 i. Halbw. Jara. Gesch. 1896 ron Direktor Angust siebert, hier.

\section{Genus 1I. Hydrophis Daud.}

1. Hydrophis caerulescens (Shaw).

B oulenger, ('at. III pag. 275.

4275,1a. Halbw. Madras. Gesch. 1889 ron Theodor Kolb, daselhst.

2. Hydrophis gracilis (Shaw).

Boulenger, Cat. III pag. 280; Boettger. Ber. Offenbach. Ver. f. Naturk. 1892 pag. 89.

9280 a. Halbw. Madras. Gesch. 1890 von Theodor Kolb, dlaselbst 9280 b. Erw. Insel Hainan, Siid-China. Gek. 1886 von Otto Herz, St.-Petersbur'w. 
3. Hydrophis cantoris Gthr.

Boulenger, ('at. 111 pag. 281, Taf. 14; Boettger, Ber. Senck.

Nat. Ges. 1889 pag. 304.

9281 a. Erw. u. halbw. Madras. Gesch, 1889 ron Theodor Kolb. rlaselbst.

\section{Hydrophis fasciatus (Schnd.).}

B o n lenger, C'at. III pag. 281; Bocttger, Ber. Offenbach. Ver. f. Naturk. 1892 pag. 89 nnil $15 \bar{n}$.

9281,1 a. Jumg. Bai ron Janila, Lu\%on. Gesch. 1891 von Konsul Dr. O. Fr.

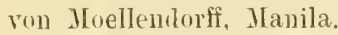

9281,1 b. Erw. ठ. Yaeyama auf Mijakoshima, Niilliche Linkin-Inseln. Gesch. 1895 von Bernhard schmacker, shaughai.

\section{Genus III. Distira Lacép.}

1. Distira orinata (Gray).

B oulenger', ('at. III pag. 290); Boettger, Zool, Anzeiger 1888 pag. 397 (Hydrophis).

9290 a. 2 hallsw. Siid-Luzon, Philippinen. Gesch. 1888 ron Konsul Lr. (). Fri, von Moellenilorff, Manila.

9290 b. 2 erw. 3 jung. MitteI-Inzon. (iesch. 1890 rom demselben.

y.200 c. Zahlr. Ste. Bai von Janila, Luzon. Gesch. 1891 ron demselben.

2. Distira brugmansi (Boie).

Boulenger, Cat. 111 pag. 292.

9.29.2 a. Erw. ठ. Madras. Gesch. 1890 ron Theodor Kolb, daselbst.

9:92.?․ Erw. ․ Ebendaher. Gesch. 1890 ron demselben.

3. Distira cyanocincta (Daud.).

Boulenger. Cat. III pag. 294; Boettger, Ber. Offeubach. Ver, f. Naturk. 1892 pag. 90.

9295 a. Erw. (var. A: Bombay. Gesch. 1888 von Prof. Dr. Ferdinand Richters. licer.

9295 b. Halbw. (var. D). Batavia, West-Java. Gesch. 1894 von Prof. Dr. K. IV. Semon. Jena.

9295 c. Erw. ठ var. B). Bai ron Manila, Luzon. Gesch. 1891 ron Konsul Dr. 0. Fr. von ILeellendurff, Manila.

929ód. Erw. o (var. D). Insel Hainan, Süd-c'hina. Gek. 1885 von Otto Herz, st.-Petersburg.

9295e. 6 halbw. Ste, (var. B). Bai von Manila, Luzon. Gesch. 1888 von Konsul Dr. O. Fr. von Jloellendorff.

9295 f. Erw. u. 4 Junge (var. A). See Taal, Luzon. Gesch. 1897 ron demselben. 


\section{Distira viperina (Sclimidt).}

Boulenger, Cat. III pag. 298; Boettger, Ber. Offenbach. Ver.

f. Naturk. 1888 pag. 91 (Hydrophis).

9298 a. Erw. ․ Insel Hainan, Sïil-China. Gek. 1886 ron Otto Herz, St.-Petersburg.

$$
\text { 5. Distira jerdoni (Gray). }
$$

Boulenger. Cat. III pag. 299; Boettger. Mitth. Geogr. Ges. u. Nat. Mns. Lübeck (2) Bd. 5, 1893 p.

9299 a. Erw. Ceylou. Gek. 1889 ron Hans Fruhstorfer; Berlin.

\section{Genus IT. Euhydris MerT.}

1. Enhydris hardwickei (Gray).

Boulenger, Cat. III pag. 301: Boettger. Zool. Anzeiger 1888 pag. 396 (Hydrophis loreatus).

9301 a. 6 Ste. Bai ron Manila, Iuzon. Gesch. 1891 von Konsul Dr. O. Fr. von Moellendorff, Manila.

\section{Genus I. Enhydrina Gray.}

1. Enhydrina valakadien (Boie).

Boulenger, Cat. III pag. 302.

9302 a. Erw. (Rïppell, (at. III Y 1 a als Hydropluis schistosus). Indischer 0сеаn. Gesch. 1836 von Dr. merl. Eduard Rüppell, hier.

9302 b. 2 erw. Madras. Gesch. 1890 ron Theodor Kolb, daselbst.

\section{Genus VI. Aepysurus Lacép.}

2. Aepysurus eydouxi (Gray).

Boulenger, Cat. III pag. 304; Boettger, Zool. Anzeiger 1892 pag. 420 (anguilliformis).

9304a. Erw. Java. Gesch. 1896 von Direktor August Siebert, hier.

9304 b. Jung. Singapore. Gesch. 1892 ron Prof. Dr. O. Boettger, hier.

\section{Genus VII. Platurus Daud.}

1. Platurus laticaudatus (L.).

Boulenger, Cat. III pag. 307.

9307 a. Halbw. Patani auf Halmalıera, Jolukken. Erh. 1895 von der Rüppellreise Prof. Dr. WV. Kü̈lienthals, Jena.

9307 b. Hallow. Insel Teruate. Erh. 1895 ron derselben.

9307c. Halbw. Atschin, Nord-Sumatra. Gesch. 1891 ron Prof. Dr. 0. Boettger, hier. 
2. Platurus colubrinus (Schnd.).

B oul enger, C'at. III pag. 308.

9308 a. Halbw. Insel Ternate. Erh. 1895 ron der Rüppelheise Prof. Dr. W.

Kiikenthals, Jena.

9308 b. 2 halbw. Manila auf Luzon. Gesch. 1890 von Konsul Dr. O. Fr. von Moellendorff, daselbst.

9308c. Halbw. Neuholland. Gesch. 1862 von Adam Becker: Sydney.

9308 d. Erw. Siidsee. Gek. 1887 von C. A. Pöhl, Hamburg.

9308 e. Halbw. Tonga-Tabu, Tonga-Inseln. Gek. 1877 von Hamburg.

9308 f. 2 J, 4 \%. Insel Cebú, Philippinen. Gesch. 1890 von Konsul Dr. 0.

$F_{1}$. von Hoellendorff.

\section{Subfamilie \%. Elapinae.}

Genus I. Ogmodon Pts.

1. Ogmodon vitianus Pts.

B oulenger, Cat. III pag. 313.

9313 a. Erw. u. jung. Viti Levu, Filji-Inselı. Gek. 1887 von C. A. Pöhl,

Hamburg.

Genus II. Pseudelaps D. B.

1. Pseudelaps muelleri (Schlg.).

Boulenger, Cat. III pag. 316; Boettger in Semon's Zool. Forsch. Heft 5, 1894 pag. 121 (Diemenia).

9316 a. Erw. Siidost-Nenguinea. Gesch. 1894 von Prof. Dr. R. W. Semon, Jena.

2. Pseudelaps harriettae (Kreffit).

Boulenger, Cat. III pag. 318.

9318,2 a. Erw. Queenslaul. Gesch. 1872 von Konservator Adam Koch, hier.

3. Pseudelaps diadema (Schlg.).

Boulenger, Cat. III pag. 319 .

9319 a. Halbw. Port Bowen, Nord-Australieu. Gek. 1887 von C. A. Pöhl, Hamburg. 


\section{Gemus III. Diemenia Gray.}

\section{Diemenia maculiceps n. sp.*)}

Boettger in Semon's Zool. Forsch. in Anstr. Heft 5, Schlangen. 1894 pag. 118 (olicacea, nom Gray).

9321 a. Erw. Bumett River, Queemsiand. Gesch. 1894 ron Prof. Dr. R. W. Semon, Jena.

2. Diemenia psammophis (Schlg.).

Boulenger. ('at. III pag. 32.2; . Tan, Icon. 1)ph. Lief. 43, Taf. 3. Fig. 3 (Pseudelaps).

932-2 a. 2 halbw. ('Tyums). Sylney. Neusiidwales. Gesch. 1862 ron Adam Becker, daselbst.

*) Tächstrerwandt der 1). psammopltis (Schlg.). aber mit mur 66-69 Subcaudalen. auf der sclmauzenspitze weiter nach binten ïhergebogenem und hier zugespitztem Rostrale, relativ kleineren Internasalen und wesentlich anderer Kopfzeichmong. - Ange grof. Rostrale so linch wie lireit, sein oberer Teil so lang wie dic Internasalsntur; Intermasalen halh so lang wie die großen Praefrontalen: Frontale nicht breiter als das einzelne snpraoculare, $2^{1 / 4}$ mal so lang wie rome hreit, so lang wie sein Abstand rou der Schnanzenspitze uni so lang wie die Parietalen. Yasale geteilt, praenasaler Teil vorn doppelt so hoch wie die hïchste Erhebung des postnasalen Teiles, in Berïhrung mit dem einzigen Praeocnlare: zwei Postocularen. Temporalen $2+2$, das untere vordere zwischen dem fünften uni sechsten supralabiale eingeschoben. Sechs supratabialen. ron denen das dritte und las vierte das Ange beriihren; drei Infralabialen in Berührung mit den vorleren Kimschillern, lie wesentlich kürzer sinıl als die hinteren.

Schuppenformel: Sipn. 15: G. t, V. 175-176, A. ${ }^{1} 1$, Sc. ${ }^{65} / 66-{ }^{69} 69+1$.

Oben olivengrum. jedle Schuppe mit schwarzer Spitze und mit schmalen schwarzen Käumen, riele nit weißen AnBenrändern, was ler Zeichnnng ein engmaschiges Aussehen gieht. Kopfschiller mit schmalen schwarzen Sämmen mo je einem schwarzen Rundfleckn anf den Praefrontalen und je zweien anf den supraocularen: Rostrale ohne dunkle Querlinie: Ange ron cinem gelblichen Kreis umgeben, ¿ler von einem geschlossenen schwarzen Kreise unralme wiri: sechstes Supralabiale und vorideres unteres. Temporale mit je einem runden schwarzen Hittelflecken. Interseite graugrim, die Ventralen an ilen Hinterändern seitlich leicht angedunkelt; Kopf- und Halsunterseite und die letzte Hälfte des Schwanzes nnten chromgelb. - Die Schwarzfleckung im L'mkreis des Anges läßt sich in keiner Weise anf die so charakteristische Zeichnung bei $D$. psammophis (Schlg.) zurïckführen, was mich in erster Linie bestimmt hat, die Art von ihr abzntrennen.

II a B e: Totallänge 820 , Schwanzlänge $172 \mathrm{~mm}$. 
9322 b. Halbw. (var. reticulata Gray). Qquensland. Gek. 1887 ron C.A. Töll, Hamburg.

9322 c. Erw. (Typus). Yeusidwales (Original ron lan's oben citierter thbillung). Alte sammlung 1845 .

$$
\text { 3. Diemenia textilis (D. B.). }
$$

Boulenger, Cat. III pag. 325.

9325 a. Erw. u. jung. Syilney. Yeusiidwales. Gesch. 1844 rou Konsul Kirchner. Wiesbalen, und 1862 ron Arlam Becker, syilney.

\section{Gems II. Pseudechis Wgl.}

1. Pseudechis porphyriacus (Shaw).

B o u l enger, Cat. III pag. 328.

9328a. Erw. (Rïppell, Cat. III O 18 a als Coluber porphyreus). Seuhollamd. Get. 1838 rom Xaturl. Hofmusenm, Wien.

\section{Genus Tr. Denisonia Krefft.}

1. Denisonia corunata (Schlg.).

Boulenger, ('at. III pag. 335̃ J an. Icon. Ophird. Lief. $4 t$, Tat. 1, Fig. 3 (Alecto).

9335a. Erw. West-Australien Uriginal zu Jan's uben vitierter Abbildung: Gesch. 186iz von Adam Becker, Sydney.

2. Denisonia siguata (Jan).

Boulenger, Cat. III pag. 338.

9338,2 a. Erw. n. jung. sychey, Mensidwales. Gesch. 1847-62 ron Konsul

W. Kirchner, IIiesbaden, und Adam Becker, sydney.

3. Denisonia daemeli (Gthr.).

Boulenger, Lat. III pag. 339, Taf. 18, Fig. 3.

9:339 a. Errv. Queeuslanıl. Gek. 1887 von C'. A. Pöhl, Hamburg.

4. Denisunia maculata (Stdchr.).

Bonlenger. Cat. 111 pag. $3+1$.

9341 a. Erw. (quemslund. Gek. 1887 vou C'. A. Pöhl, Hambnrg. 


\section{Genus VI. Micropechis Blgr.}

1. Micropechis ikaheka (Lesson). Boulenger, Cat. III pag. 347.

$93 \pm 7$ a. Halbw. Nenguinea. Gesch. 1897 von Prof. Dr. O. Boettger, hier.

Genus VII. Hoplocephalus Cur.

1. Hoplocephalus bitorquatus (Jan).

Boulenger, Cat. III pag. 349; Jan, Icon. Oph. Lief. 43, Taf. 6,

Fig. 6 (Alecto).

9349 a. Erw. Sydney, Neusiudwales (Originalexemplar dieser Art; vergl.

J an, Rer. et Mag. Zool. 1859 pag. 128 und l. c.). Gesch. 1848 von Konsul W. Kirchner, Wiesbaden.

\section{Genus VIII. Acronthophis Daud.}

1. Acanthophis antarcticus (Shaw).

Boulenger. Cat. III pag. 35̃ó; Boettger, Ber. Offenbach. Ver. f. Naturk. 1892 pag. 1 วั.

935̄ a. Erw. Key-Iuselı. Gesch. 1887 von Prof. Dr. Max Braun, Rostock. 9355 b. Erw. Stadt Haruku, Insel Harnku bei Ceram. Gesch. 1890 ron Dr. Adolf Strubell, Bomn.

9355 c. Erw. (gestopft). Neuholland (Rüppell, Cat. III HH 1 a als cerastinus). Get. 1832 rom British IIuseum (N. H.), London.

\section{Genus IX. Elapechis Blgr.}

1. Elapechis guentheri (Boc.).

B oulenger, Cat. III pag. 359; B oettger, Ber. Senck. Nat. Ges. 1888 pag. 82 (Elaysoidea).

9358a. 2 erw. Poro Nemlao bei Banana. Unter.Congo. Gek. 1886 von Paul Hesse, Venedig.

2. Elapechis hessei (Bttgr.).

Boulenger, C'at. III pag. 360; Boettger', Zool. Anzeiger 1887 pag. 651 und Ber. Senck. Nat. Ges. 1888 pag. 83, Taf. 2 , Fig. 6 (Elapsoidea).

9360 a. Halbw. Poro Netonna bei Banana, Unter-Congo. Gek. 1886 ron Paul Hesse, Venedig. 
3. Elapechis boulengeri (Bttgr.).

Boulenger, Cat. III pag. 361; Boettger, Zool, Anzeiger 1895 pag. 92 (Elapsoidea).

9361 a. Halbw. Boroma am siumbesi. Gescl. 1895 von Prof. Dr. O. Boettger, hier.

\section{Genus X. Bungarus Daud.}

1. Bungalus fasciatus (Schnd.).

Boulenger, Cat. III pay. 366; B o ettger, Ber. Offenbach. Ver. f. Naturk. 1885 pag. 16 und 1888 pag. 86.

Y366 a. Erw. Java. Gek. 1879 von Hamburg.

9366 b. Erw. Baramfluß in Nord-Borneo. Erh. 1895 von der Rüppellreise Prof. Dr. IV. Kuikenthals, Jena.

9366 c. Erw. u. jung. Buitenzorg, West-Java. Gesch. 1890 von Dr. Adolf Strubell, Bonn.

9366 rl. Erw. Stabat Estate, Sultanat Deli, Nor(lost-Sumatra. Gesch. 1893 von Fritz Beyschlag, daselbst.

9366 e. Erw. (Riippell, Cat. III Z 1 a als annularis). Jara. Gesch. 1838 von Dr. W. von Siebold, Leirlen.

9366 f. Erw. Kanton, Süd-China. Gesch. 1885 von Konsul Dr. O. Fr. von Moellendorff, Manila.

2. Bungarus ceylonicus Gthr.

Boulenger, Cat. III pag. 367, Taf. 19, Fig. 3.

9367 a. Erw. u. 2 Junge. Ceylon. Gesch. $18 \pm 6$ von H. Worms, hier.

3. Bungarus caudidus (L.).

B oulenger, ('at. III pag. 368; B o ettger', Ber. Offenbach. Ver. f.

Naturk. 1885 pag. 16 (semifasciatus) und 1888 pag. 86 (multicinctus).

9368 a. Erw. u. jung. Reich Jolo, Java. Gesch. 1868-86 ron E. Lörsch und Oberlehrer J. Blum, hier.

9368 b. Erw. (Rüppell, Cat. III Z 2 c als semifasciutus). Java. Gesch. 1832 von Dr. WV. von Siebold, Leiden.

9368 c. Erw. Java. Geli. 1887 von C. A. Pölll, Hamburg.

9368 i. 4 Ste. Buitenzorg. West-Ja va. Gesclı. 1890 von Dr. Adolf Strubell, Bonn. 9368 e. 2 halbw. (Riippell, ('at. III Z $2 a-b$ als semifusciutus). Java. Gesch. 1838 von Dr. merl. Peitsch, Batavia.

var. multicincta Blytl.

Boulenger, l. c. pag. 369 .

9369 a. 2 erw. Kanton, Siill-China. Gesch. 1885 ron Konsul Dr. (1. Fr. ron Joellendorff, Manila.

9369 b. 2 erw. Ebendalier. Gesch. 1886 von demselhen und Otto Herz, St.-Petersburg. 


\section{Bungarus flaviceps Reinh.}

Boulenger, Cat. III pag. 371.

9371 a. Erw. (var. A). Sultanat Deli, Nordost-Sumatra. Gesch. 1891 ron Fritz Beyschlag. daselbst.

\section{Genus XI. Naja Laur. \\ 1. Naja liaje (L.).}

Bonlenger, Cat. III pag. 374; Boettger, Abl. Senck. Nat. Ges. Bd. 13, 1883 pag. $10 t$.

937t a. Halbw. (Trpus). Ïgypten. Gesch. 1886 ron Prof. Dr. O. Boettger, hier. 4374b. Elw. (Typus). Ägyten Riippell. (at. III Ad 1 a). Gesch. 1828 rou Dr, med. Eduard Rïppell. hier.

9374 e. Erw. (Typus). Ebeudaher (Rïppell, ('at. III Ad 1b). Gesch. 1828 ron ilemselben.

937td. Erw. (var. C'. Tanger, Marokko. Erh. 1881 von der IV. Rüppellerise Dr. med. Wr. Kohelt's, Schwanheim Main).

9374 e. Jung (Typus). Ïgroten (Rüppell, Cat. IlI Ad 1 c). Gesch. 1828 von Dr. med. Eduarı Rë̈ppell.

937tf. Erw. (rar. C). Ebendaler. (iesch. 1896 von der Nenen Zoolog. Gesellschaft, hier.

937tg. Erw. (Typus). Eloudaher. Gesch. 1896 ron derselben.

937th. Erw. (Typus). Ebendaher. Gesch. 1897 ron derselhen.

\section{Naja melanolenca Hallow.}

Bonlenger. Cat. IlI pag. 376; Boettger, Ber. Senck. Nat. Ges. 1888 pag. 80 ihaje rar..

9377 a. Erw. n. jung (Tฐpus). Banana, C'uter-Congo. Gek. 1887 ron Paul Hesse, Tenedig.

9377 b. Erw. (Typus). Massabé. Loangokiiste, West-Afrika. Gek. 1887 ron demselben.

9377 c. Jung (var. B). Cabiutla, Inter-('ons"*. Gek. 1885 von demselben.

\section{Naja nigricollis Reinh.}

Boulenger, Cat. III pag. 378; Boettger, Ber. Senck. Nat. Ges. 1888 pag. 81 .

9378a. Kopf (Typus). Bras-River, West-Atrika. Gesch. 1887 ron Prof. Dr. Oskar Boettger, hier.

9378 b. Kopf (Typus). Ambrizette, Ingola. Gek, 1887 ron Panl Hesse, Venedig. 9378c. Halbw. (var. pallide Plgr.). Oher-igypten lïippell. Cat. III AA 1 d ats haje). (iesch. 1828 ron Dr. med. Ednard Rïppell. hier. 


\section{Naja tripudians Merr.}

Boulenger, Cat. III pag. 380; Boettger, Zool. Jahrb. Brl. 3 . 1888 pag. 943

9381 a. Erw. (Typus). Madras. Gesch. 1889 von Theorlor Kolb, rlasellst. 9:81 b. Erw. (Trpus). Bengalen (Rüppell, Cat. III AA 2a). Get, 1838 rom Nat. Rijksmuseum in Leiden.

9381 c. Erw. (Gestopft; typus). Ostindien. Gesch. 1871 von Phil. Friedr. Behrends, hier.

9381 ¿. Erw. (Gestopft: typus). Ehendaher. Alte Sammlung 1845.

9381 e-f. 2 erw. (Gestopft; typus). El)endaher. Gek. 1846 ron Boissonean.

\section{var, caeca Gimel.}

B o n lenger, l. c. pag. 382.

9382 a. Erw. Mittel-Luzon, Philippinen. Gesch. 1890 von Konsul Lr, O. Fr. ron Moellendorff, Manila.

9382 b. Erw. Brunnen Beschlerma am Kopet-dagh. Transkaspien. Gesch. 1888 ron Staatsrat Dr. Gustar von Rarlde. Excz., Tiflis.

var, fasciata Gray.

Boulenger, Cat. III pag. 383.

9383 a. Erw. (forma b). Kanton, Siid-('hina. Gesch. 1885 ron Kousul Dr. O. Fr. yon Mloellendorff. Manila.

9383 b. Erw. (dieselbe Form). Ehendaher. Ciesch. 188 a ron demselben. 9383 c. Erw. (dieselbe Form). Insel Hainan, Sïd-(hina. Gek. 1886 von roto Herz. St.-Petersburg.

val. sylutatrix Boie.

Boulenger, Cat. III pag. 384.

9384 a. 2. Iunge. Bnitenzorg, West-Java. Gescl. 1890 von Dr. Arlolf Strubell. Bomn.

$938+$ b). 2 halbw. (Rïppell, ('at. III AA 3 a-b). Java. Gesch. 1838 von Dr. W. von Siebold. Leiden.

var. leucoulira Blgr.

Boul lenger. 1. c. pag. 384 .

9384.1 a. Jung. Java. Gesch. 1896 von Direktor Angnst Siebert, hier.

9384,1 b. Erw. Sultanat Deli, Nordost-Simnatra. Gesch. 1886 von r'lemens Hohwiesner, hier.

9384,1 c. Jung. Ehendaher. Gesch. 1892 ron Inr. med. (O. Benecke, daselbst. 


\section{Naja samareusis Pts.}

Boulenger, Cat. III pag. 385.

9385 a. Erw. u. jung. Insel Samar, Philippinen. Gesch. 1897 von Konsul Dr. O. Fr. von Moellendorff, Manila.

\section{Naja bungarus Schlg.}

Boulenger, Cat. III pag. 386; Boettger, Ber. Offenbach. Ver.

f. Naturk. 1888 pag. 86 (Ophiophagus elaps).

9386 a. Kopf u. Schwanz (var. C). Mittel-Luzon, Philippinen. Gesch. 1888 von Konsul Dr. O. Fr, von Moellendorff, Manila.

9386 b. Jung (var. B). Singapore. Gesch. 1896 von Dr. Heinrich Lenz, Luibeck.

\section{Genus XII. Hemibungarus Pts.}

\section{Hemibungarus fasciatus (Pts.).*)}

Peters, Mon.-Ber. Berlin. Akad. 1861 pag. 689 (Hamadryas).

9392,1 a. 3 halbw. Mranila, Luzon. Gesch, 1888-90 von Konsul Dr. O. Fr. von Ioellendorff, daselbst.

\section{Hemibungarus calligaster (Wgm.).}

Boulenger, Cat. III pag. 393.

9393 a. 4 erw. Mittel-Luzon. Gesch. 1890 ron Konsul Dr. O. Fr. von Moellendorff. Manila.

*) Hinter dlen beiden großen Giftzähnen im Oberkiefer noch ein kleines solides Zähnchen. - Rostrale etwas breiter als lang; Frontale $1^{1 / 3}$ mal länger als breit, so lang oder etwas länger als sein Abstand von der Schnauzenspitze, viel kürzer als die Parietalen; ein Praeoculare und zwei Postocularen; Temporalen $2+2$. Sechs Supralabialen, das dritte und das vierte in Beriihrung mit dem Auge; 4 Infralabialen in Kontakt mit den vorderen Kinnschildern, die viel länger sind als die linteren.

Schuppenformeln: $\delta$ Syn. 15; G. 3, V. 257, A. 1, Sc. ${ }^{20} / 20+1$,

$$
\begin{aligned}
& \text { Q }, 15 ;, 3,225,, 1 .,{ }^{19} / 19+1 \text {, } \\
& \text { ㅇ }, 15 ;, 3, n 229, n 1, \quad{ }^{23} / 23+1 \text {. }
\end{aligned}
$$

Rosa mit 26-32 schwarzen Vollringen anf Kopf und Rumpf und zwei auf dem Schwanze, die oben etwas breiter, unten schmäler sind als die hellen Zwischenräume. Der erste Ring ist ein Halbring und zieht quer iiber die Augengegend von Lippe zu Lippe, der zweite ist breiter als die übrigen und steht hinter den Parietalen, deren Spitze oder Nalıt er noch einschließt. Die schwarzen Ringe zeigen auf dem Bauche manchmal die Tendenz in Doppelringe zu zerfallen, indem sie häufig in der Mitte eine helle Qnerzone einschließen.

\aße: Totallänge 201, Schwanzlänge $12 \mathrm{~mm}$. 
var. gemmiannulis Pts.

Peters, Mon.-Ber. Berlin. Akad. 1872 pag. 587 (spec.).

9393,1 a. Erw, Mittel-Luzon. Gesch. 1890 von Konsul Dr. O. Fr. vou Moellendorff, Manila.

3. Hemibungarus japonicus (Gthr.).

B oulenger, Cat. III pag. 395; Fritze, Zool. Jahrb. Bd. 7,-189t pag. 861 (Callophis boettgeri).

9395 a. Erw. (Original von C. boettgeri Fritze). Okinawa, Liukiu - Inseln. Gek, 1896 von Naturalienhändler Gustav Schneider, Basel.

Genus XIII. Callophis Gray.

1. Callophis gracilis Gray.

Boulenger, Cat. III pag. 396.

9396 a. Erw. Sultanat Deli, Nordost-Sumatra. Gesch. 1886 von Clemens Hohwiesner, hier.

2. Callophis macelellandi (Reinh.).

B oulenger, Cat. III pag. 398.

9398 a. Erw. (var. univirgata Gthr.). Darjeeling, Ost-Himalaya. Get. 1891 rom British JIuseum (N. H.), London.

Genus XIV. Doliophis Girard.

1. Doliophis bivirgatus (Boie).

Bonlenger, Cat. III pag. 400.

9400 a. Erw. (Typus), Sultanat Deli, Nordost-Sumatra. Gesch. 1894 von Dr. med. C'arl Flach, Aschaffenburg.

9400 b. Erw. (var. flaviceps Cant.). Tenggergebirge in $1200 \mathrm{~m}$, Ost - Java. Gek. 1891 von Hans Fruhstorfer, Berlin.

9400 c. Erw. (var. tetrataenia Bleek.). Borneo. Gesch. 1886 von Prof. Dr.

O. Boettger, hier.

9400 d. 2 erw. (var. flaviceps Cant.). Binljey in Deli, Nordost-Sumatra. Gesch. 1890 von Theodor und Egbert Engelhard, daselbst.

9400 e. Erw. (var. teirutaenia Bleek.). Java (Rïppell, Cat. III EE 3 a als Elaps).

Gesch. 1838 von Dr. W. von Siebold, Leiden. 
2. Doliophis intestinalis (Laur.).

Boulenger, Cat. III pag. 401; Jan. Icon. Oph. Lief. 43. Taf, 1. Fig. $3 \mathrm{z}$ (Eleps furcatus).

9401 a. Erw. (Rüppell, Cat. III EE + b) als Elaps furcatus; Original zu Jan's oben citierter Zeichnung). Java. Gesch. 1838 von Dr. W. ron Siebold, Leiden.

9401 b. Erw. Samarang auf Java. Gesch. 1876 von Jajor Dr. L. von Heyden. hier.

9401 c. 4 Ste. Buitenzorg. West-Javil. Gesch. 1890 ron Lr. Adolf Strubell. Bonn.

\section{var. annectens Blgr.}

B o ul lenger, Cat. III pag. 402.

9402 a. Halbw. Insel Bangka. Gek. 1881 ron Konrad Kläsi, Zürich.

var. lineata Gray.

Boulenger. l. c. pag. 403.

9403 a. Erw. Stabat Estate in Deli, Nordost-Sumatra. Gesch. 1893 von Fritz Beyschlag, daselbst.

940:3 1). Erw. Atschin, Nord-Sumatra. Gek. 1882 ron Konrar Kläsi, Zürich.

3. Doliophis bilineatus (Pts.).

Boulenger. C'at. III pag. 404: B o ettg er, Abh. Zool. Mlus. Dresden 18.94-..95, To. 7 pag. ó (Alleniopleis).

9404a. Erw. Insel Palawau, Philippinen. Gesch. 1888 ron Konsul Dr. 0. Fr. ron Jroellendorff, Janila.

9404 b. Erw. Insel Culion, Calauianes, Plilippinen. Gesch. 1897 ron demselben.

4. Doliopluis philippinus (Gthr.).

Boulenger, Cat. III pag. 404.

9105 a. Erw. Insel Mindana0, Philippinen. Gesch. 1888 ron Konsul Dr. O.

Fr. von Hoellendorff, Janila.

Genus XT. Furina D. B.

1. Furiua occipitalis (D. B.).

Boulenger. Cat. III pag. 407.

9408 a. Erw. Tenholland. Gesch. 1881 ron J. C. Parrot, hier.

9408 b. Erw. Sydney, Teusidlwales. Gescll, 1848 ron Konsul W. Kirchner: Wiesbaden. 


\section{Genus XVI. Homorelaps Jan.}

1. Homorelaps lacteus (L.).

B oulenger', ('at. IJI pag. 409.

9409 a. Halbw. (var. punctute Smith). Siid-Afrika. Gesch. 1897 ron Prof. Dr. (1. Boettger, hier.

\section{Genus XYII. Elaps Schnd.}

1. Elaps surinamensis Cur.

B oulenger. Cat. III pag. 414.

9413 a. Erw. Zwischen Guaramaco mu San Fernanio. Alto Grenoco, SiidVenezural. Gek. 1895 vou Georg Hïbner, Dresden.

2. Elaps heterozonus Pts.

Boulenger. Cat. III pag. 417.

9417.1a. 2 halbw. Sorata, Boliria. Gesch. 1891 von Dr. Heinrich Lenz. Liibeck.

3. Elaps corallinus Wied.

Boulenger, (at. III pag. 420); Boettger, Ber. Senck. Nat. Ges. 1889 pag. 31 (var. gastrosticta).

9420 a. 2 elw. (Typus). Pacasmayo. Nord-P'ro. Gesch. 1887 von Max Banberger, hier.

9420 h. 4 erw. (Trpus). Ehendaher. Gesch. 1889 von demselben.

9420c. Jung (Typus). Peru. Gesch. 1863 von Feldmarschall Philipp Braum. Lima.

9420 d. Erw. u. 2 .Junge (Typus). Trinidad. Gesch. 1879 ron ('arl Knoblanch, hier.

9420 e. Erw. (rar. B). Não Paulo, Brasilien. Gesch. 1890 von Prof. Dr. O. Boettger, hier.

9420 f. 2 erw. (Trpus). Trinidad. Fesch. 1893 ron F. W. Trich unil R. R. IIole, Port of Spain.

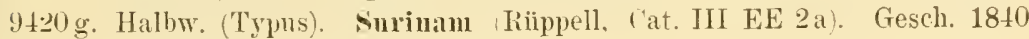
ron Dr. med. Wr. Sichel, hier.

9420 h. Jung. Tropisches Niid-Amerika. Gek. 1887 von (C. A. Pöhl, Hamburg. 9420 i. Erw. o (Varietät mit nur 12 schwarzen Körperringen). Baranquilla in Magdalenathal, U. S. Colmubia. Gek. 1897 ron Prof. Dr. Fritz Regel. Jena.

4. Elaps tschudi Jan.

Boulenger, Cat. III pag. 422; Boettger; Ber. Senck. Nat. Ges. 1889 pag. 316.

9422 a. Halbw. Pacasmayo, Nord-Peru. Gesch. 1887 von Jax Bamberger, hier. 
5. Elaps dissoleucus Cope.

B o ulenger, Cat. III pag. 422.

9422,1 a. 2 halbw. Baranquilla im Magdalenathal, U. S. Columbia. Gek. 1897 von Prof. Dr. Fritz Regel, Jena.

$$
\text { 6. Elaps fulvius (L.). }
$$

Boulenger, Cat. III pag. 422.

9423 a. Erw. Siidliche Verein. Staatell. Gesch. 1848 ron Dr.med. W. Sichel, lier.

9423 b. Erw. Florila. Gescl. 1896 von Dr. mer. A. Zipperlen, Cincinnati, O.

var. nigrocincta Gir.

B oul eng er, l. c. pag. 424 .

9424 a. Erw. Retallulen, Guatemala. Gesch. 1895 von Carl Fleischmann, Guatemala.

9424 b. Erw. u. jung. San José, Costa Rica. Gesch. 1893 von demselben.

\section{Elaps spixi (Wgl.).}

Boulenger, ('at. III pag. 427.

9427a. Erw. Mapiri, NebenfluB des oberen Beni, Bolivia. Gesch. 1888 von Ferdinand Emmel, Arequipa.

9427 b. Erw. Ciudar Bolivar, Yenezuela. Gesch. 1894 von F. W. Urich und R. R. IIole, Port of Spain.

9427 c. Erw. Carácas. Venezuela. Gek. 1897 von Ingenieur Müller, hier.

$$
\text { 8. Elaps froutalis D. B. }
$$

Boulenger, Cat. III pag. 427.

9427,1 a. Erw. (var. B). Siid-Brasilien. Gek. 1877 von Hamburg.

9. Elaps marcgravii Wied.

Boulenger, Cat. III pag. 428.

9428 a. 3 erw. (Rüppell, Cat. III EE 1 a-c). Illeos, Brasiliell. Gesch. 1838 von Gebr. Koch, hier.

9428 b. 3 halbw. (Rüppell, Cat. III EE 1d-f). Brasilien. Get. 1836 rom Herzog von Leuchtenberg.

9428 c. Erw. Ilheos, Brasilien. Gesch. 1838 von Gebr. Koch, hier.

9428 d. Erw. Trinidad. Gesch. 1893 ron F. W. Urich und R. R. Jlole, Port of Spain. 
10. Elaps mipartitus D. B.

Boulenger, Cat. III pag. 431.

9431 a. Erw. Yeneznela. Gek. 1877 von Hamburg.

9431 b. Erw. Amalti, C. S. Columbia. Gek. 1897 von Prof. Dr. Fritz Regel, Jena.

Genus XVIII. Dendraspis Schly.

1. Dendraspis viridis (Hallow.).

Boulenger, Cat. III pag. 43う; Boettger, Ber. Senck. Nat. Ges. 1887 pag. 63 (jamesoni).

9435 a. Erw. Akkra, Goldküste. Gesch. 1887 von Major Dr. L. von Heyden, hier.

9435 b. 2 erw. Odumase, Östl. Goldkiiste. Gesch. 1892 von Hans Simon, Stuttgart.

2. Dendraspis jamesoui (Traill).

Boulenger, Cat. III pag. 436 ; B o ettger, Ber. Senck. Nat. Ges 1888 pag. 85.

9436 a. Erw. Massabé, Loangokiiste, West-Afrika. Gek. 1887 ron Paul Hesse, Venedig.

9436 b. Kopf. Cabinda, Unter-Congo. Gek. 1887 von demselben.

9436 c. Erw. Poro Netonna bei Banana, Unter-Congo. Gek. 1887 von demselben.

\section{Familie VIII. Amblycephalidae.}

Genus I. Haplopeltura D. B.

1. Haplopeltura boa (Boie).

B oulenger. Cat. III pag. 439: Boettger, Ber. Offenbach. Ver. f. Naturk. 1892 pag. 134.

9439 a. Erw. West-Java. Gek. 1893 von Hans Fruhstorfer, Berlin.

9439 b. Erw. Buitenzorg, West-Jara. Gesch. 1890 von Dr. Adolf Strubell, Bonn.

Genus II. Amblycephalus Kuhl.

1. Amblycephalus laevis Boie.

Boulenger, Cat. III pag. 441.

9441 a. Halbw. Sultanat Deli, Nordost-Sumatra. Gesch. 1881 von Fritz Beyschlag, daselbst. 
9441 b. Halbw. Baramfluß in Nord-Borneo. Erh. 1895 von der Rüppellreise Prof. Dr. Wr. Kïlienthals, .Tena.

$94+1$ c. Erw. und jung. Buitenzorg, West-Java. Gesch, 1890 von Dr. Adolf Strubell, Bomm.

\section{Amblycephalus moellendorffi (Bttgr.).}

Boulenger, Cat. III pag. 443 ; Boettger, Ber. Uffenbach. Ver. f. Taturk, 1885 pag. 125 und 1888 pag. 84, Taf. 2, Fig. 1 (Pareas). 9443.1 a. 2 erw. Gebirge Lo-fou-shau bei Kianton, Siil-China. Gesch. 188:) - 88 ron Bermhard schmacker, Shanghai. mol Lomsul Dr. O. Fr. von ILellentorfif, Ilanila.

9443,1 b. 2 elw. Hongkong, Siul-China. Get. 188 r von Bernh. Schnacker. 9443, I c. Erw. u. jung. Bangkrk, siam. Gesch. 1885 von Konsul Dr. O. Fr. ron Moellendoriff.

3. Amblycephalus carinatus Boie.

Bonlenger, ('at. IlI pag. 4t5: Boettger, Ber. Offenbach. Ver. f. Naturk. 1892 pag. 135.

9445a. Erw. (Riippell, Cat. III Y ya als Dipsus). Java. Get. 1838 rom Naturh. Rijksmuseum in Leillen.

9445 b. 2 erw., 2 jung. Buitenzorg, West-Java. Gesch. 1890 ron Dr. Arlolf Strubell, Bonn.

\section{Genus III. Leptognathus D. B.}

\section{Leptoguathus peruaua n. sp.*)}

9448 a. Erw. Sta. Ana, Prov. Cuzco, Peru. Gesch. 1890 ron Frieilich Emmel, Gelnhausen.

*) Ähnlich dem L. cutesbyi (Sentz.), aber mit ló Schuppenreihen, höherem Rostrale nnd kïrzerem Frontale. Ton $L$. mikani (Schlg.) verschieden ilurch viel kompressere Gestalt, durch ilie Zahl, Forn und stellung der Infralabialen und durch die fast ganz chmkle Körperunterseite. - Kopf stark rom Halse abgesetzt. Rnmpl sehr zusammengedrücht. in ler lö̈rpermitte fast loppelt so hoch wie tief. Ange groß. Rostrale fast so hoch wie breit, ron oben nur wenig sichtbar; Internasalen mehr als habmal so lang wie die ans Ange stoßenten Praefrontalen; Frontale etwas breiter als lang, kürzer als sein Abstanıl von der Schnauzenspritze, viel kïizer als die Parietalen. Nasale geteilt; Frenale so hoch wie lang, ans Auge stoßend; kein Praeoculare zwei Postocularen; Temporalen $2+3$. Acht Supralabialen, von denen das dritte, rierte mol fünfte ans Ange treten. 13-14 Infralabialen; das erste hinter dem symphysale in Beriilurung mit dem der andern Seite; jederseits fïnf Infratabialen in Kontakt mit dem ersten Paar lïinnschilder, die viel länger sind als breit. Tier Paar Kinnschilder. Die schuppen der mittelsten 
2. Leptoguathus cateshyi (Sentz.).

Boulenger, Cat. III pag. 449.

9449 a. Halbw. Mapiri, NehenfluB des oberen Beni, Bolivia. Gesch. 1888 von

Ferdinand Emmel, Arequipa.

9449 b. Jung. Sorata, Bolivia. Gesch. 1891 von Dr. Heinr. Lenz, Liibeck. 9449 c. 3 erw. Sta. Ana, Prov. Cuzco, Pern. Gesch. 1890 von Friedrich Emmel, Gelnhansen.

9449 ،l. 2 erw. (Riippell, ('at. III V 5̃a-b als Dipsas). Surinam. Alte Sammling $18+5$.

3. Leptognathus pavonina (Schlg.).

Boulenger, Cat. III pag. 450.

9450 a. Erw. und jung (Rüppell, Cat. III $\mathrm{V} 4 \mathrm{a}-\mathrm{b}$ als Dipsas weigeli). Ilheos,

Brasilien. Gesch. 1838 von Gebr. Koch, hier.

\section{Leptoguathus mikani (Schlg.).}

Boulenger, Cat. III pag. 453.

9453,1 a. Erw. und jung (Typus). Rio (trande do sul, Süd-Brasilien. Gek. 1886 ron Prof. Dr. H. von Ihering, daselbst.

5. Leptoguathus ventrimaculata Blgr.

Bonlenger, Cat. III pag. 454, Taf. 24, Fig. 2.

9454 a. Erw. Rio Grande do Sul, Süd-Brasilien. Gesch. 1873 von Oberlehrer Dr. Finger, hier.

Riickenreihe stark verbreitert, aber die breitesten schuppen nicht doppelt so breit wie lang.

Schuppenformel: Squ. 15 ; G. ${ }^{4} / 4$, V. 180, A. 1 , Sc. ${ }^{79} / 79+1$.

Oben dunkel rotbraun mit breiten schwarzen, weiß gesäumten, opponierenden oder altermierenden Querbinden, die bis auf die Ventralen herabsteigen; diese dunkeln Binden breiter als die hellen Zwischenräume, 34 anf lem Rumpfe bis zum After. Kopf einfarbig schwarz, nur die Lippen mit weißen Flecken. Unterseite ron Kopf, Hals und erstem Rumpfdrittel schwarz und milchweiß gewïrfelt, die der letzten beiden Rumpfdrittel und des Schwanzes fast einfarbig schwarzgrau mit sehr undeutlichen, schmalen weißliclien Längsadern.

II a B e: Totallänge 610, Schwanzlänge $155 \mathrm{~mm}$.

Fundort: Sta. Ana in der peruanischen Provinz Cuzco, ein tarlellos erhaltenes Exemplar, von Herrn Ferdinand Emmel in Arequipa gesammelt und durch dessen Bruder, Herm Friedrich Emmel in Gelnhausen, dem Museum zum Geschenk gemacht. 


\section{Familie IX. Viperidae.}

\section{Subfamilie 1. Viperinae.}

\section{Genus I. Causus Wgl.}

1. Causus rhombeatus (Licht.).

Boulenger, Cat. III pag. 467; Boettger, Abh. Senck. Nat. Ges. Bd. 12, 1881 pag. 399 (Aspidelaps), Ber. Senck. Nat. Ges. 1887 pag. 63 und 1888 pag. 88.

9467 a. Halbw. Port Natal. Gek. 1877 von Hamburg.

9467 b. Halbw. Aburi, Goldkiuste. Gesch. 1885 von Hans Simon, Stuttgart.

9467 c. 2 erw. Akkra, (xoldkiiste. Gesch. 1887 von Major Dr. L. von Heyden, hier.

9467 d. 2 erw. Kapland. Gesch. 1873 von Dr. Löwenthal, hier.

9467 e. Erw. Banana, Unter-Congo. Gek. 1885 von Panl Hesse, Venedig. 9467 f. Erw. Kinshassa am Stanley Pool, Unter - Congo. Gek. 1887 ron demselben.

9467 g. 4 erw. Banana, Unter-Congo. Gek. 1885 von demselben.

\section{Genus II. Tipera Laur.}

1. Vipera ursinii Bonap.

Boulenger, Cat. III pag. 473.

9473 a. Erw. đond $q$. Bosnien. Gesch. 1893 von Schulrat Dr. Egid Schreiber, Görz.

9473 b. Erw. Laxenburg bei Wien. Gesch. 1893 von G. A. Boulenger, F. R.S., London.

9473 c. Erw. ․ Ebendaher. Gesch. 1893 von Dr. Franz Werner, Wien.

\section{Vipera renardi Christ.}

Boulenger, Cat. III pag, 475; B oettger, Ber. Senck. Nat. Ges. 1892 pag. 149 (berus, non L.).

9475a. Erw. Stanitze Pjaetigorsk bei Jaaikop, Ciskaukasien. Gek. 1891 ron Paul Reibisch, Dresden.

9475 b. Halbw. Stadt Pjaetigorsk, Ciskankasien. Gesch. 1881 von Hans Simon, Stuttgart.

\section{Vipera berus (L.).}

Boulenger, Cat. III pag. 476; Notthaft, Zool. Anzeiger 1886 pag. 450; Bl u m, Abl. Senck. Nat. Ges. Bd. 15, 1888 pag. 128.

9476 a. Erw. ơ und o. Cibinsgebirge, Siebenbiirgen (Hohe Rinne). Gesch. 1896 von Dr. Carl Fr. Jickeli, Hermannstadt. 
9476 b. 2 erw. б. Kobyllno, Oberschlesiel. Gesch. 1886 von Prof. Dr. O. Boettger; hier.

9476 c. Jung (Typus) und erw. (var. prester L.). St. Blasien, Bad. Scliwar\%wall. Gesch. 1886 von demselben.

9476 d. Erw. Sils-Baseglia in 1920 m, Ober-Eugadiı. Gesch. 1890 von Heinrich Flinseh, hier.

9476 e. 5 erw. $\delta$ und ㅇ. Wunsiedel, Ober-Franken. Gesch. 1891 ron Oberlehrer J. Pilnm, hier.

9476f. Erw. Thiiringer Wald. Gesch. 1891 von Hospitalmeister Philipp Reichard, hier.

9476 g. Erw. (var. prester L.). Südabhang des Nebelhorns in 6000', Oberstlorf im Algäı. Gesch. 1876 von Haler Carl Dietze, Jugenheim.

9476 h. Erw. ơ und + (var. prester L.). Malborgeth, Käirnten. Gesch. 1893 von Schulrat Dr. Egiıl Schreiber, Görz.

9476i. Erw. ठ゚. Klönthal bei Richisau, Ct. Glarus, Sclıweiz. Gesch. 1891 von Oberlehrer J. Blum, hier.

9476 k. 2 erw. Mahlberg, Kreis Rees, Rheimprovinz. Gesch. 1891 ron demselben.

94761. Erw. \& und 2 of (var. prester L. und 2 Typus). Karolinenhorst, Kr. Naugard, Pommern. Gesch. 1891 von demselben.

9476 m. 3 erw. Kloster Reitberg, Kr. Tölz, Oberbayern. Gesch. 1891 von demselben.

9476 n. $2 \delta$ (Typus) und $q$ (var. prester L.). Born auf der Halbinsel Dars, Kr. Franzburg, Pommeru. Gesch. 1891 von demselben.

9476 o. Erw. ․ Orb, Kr. Gelnhausen, Hessen-Nassall. Gesch. 1891 ron demselben.

9476 p. Erw. ㅇ. Mohrungen, 0stpreussen. Gesch. 1891 von demselben.

9476 . $\delta$ und $q$ (Typus) und $q$ (var. prester L.). Weg zur Klublrütte des Glärnisch, Rossmatterthal, Ct. Glarus, Sclıweiz. Gesch. 1891 von demselben.

9476 r. Erw. O (var. prester L.). Route Psirsk-Suchum, West-Transkankasien. Erh. $188 . t$ von der Reise Staatsrat O. Retowski's, 'Theodosia.

9476 s. Halbw. Unter-Franken. Gesch. 1865 von Ferdinand Knoblauch, hier. 9476 t. Erw. (var. prester L.). WestfuB des Hohen Plassen in 6200' bei Hallstarlt, Salzkammergut. Gesch. 1876 von San.-Rat Dr. med. $\mathrm{Ph}$. G. Passavant, hier.

9476 u. Erw. ơ (var. seounei Lat.). Cabañas bei Coruña, Nordwest-Spanieu. Gesch. 1880 ron V. Lopez Seoane, daselbst.

9476 v. 3 erw. Vegesack bei Bremen. Gesch. 1886 von Prof. Dr. O. Boettger, hier.

9476 w. 2 erw. (Rïppell, Cat. III GG $1 \mathrm{a}-\mathrm{b}$ ). Hamburg. Gesch. 1838 von Schöff Dr. Carl von Heyden, hier.

9476 x. Erww. ․ Grödener Jöchel bei St. Ulrich, 'Tirol. Gesch. 1880 von Prof. Dr. Ach. Andreae, Hildesheim.

9476 y. Erw. (gestopft). Norddentschland. Alte Sammlung 1845.

9476 z. Erw. (in Formol). Siebentisch bei Augsburg. Gesch. 1897 von Oberlehrer J. Blum. 


\section{Vipera aspis (L.).}

B oulenger, Cat. III pag. 481; B l n m, Abl. Senck. Nat. Ges. Bi. 15, 1888 pag. 128 und Zool. Garten 1892 pag. 12 und 265.

9481 a. Erw. Leinegg im Schwarzathal, Seitenthal des Schliichtthals, Siidschwarzwald. Gesch. 1891 von Oberlehrer J. Blum, hier.

9481 b. Erw. $q$ mit Embryonen. Bois de Taux, Ars an iler Mosel, DentschLothringen. Gesch. 1892 von Lehrer Fr, Bastier, hier.

9481 c. 3 elw. (var. hugyi Schinz). (altanisetta, Sicilien. Gesch. 1881-86 ron Chefinspektor Carl Hirsch, Palermo.

9481 e. Erw. (Rïppell, ('at. IlI FF 4a). Siid-Europa. Alte Sammlung 1845.

9481f. Erw. (var. hugyi Schinz). Mte. Cuccio bei Palermo, Sicilien. Gesch. 1882 ron Chefinspektor Carl Hirsch.

9481 g. 2 erw. ․ Aus der Phraze zwischen Toréant und Dornot, DentschLothringen. Gesch. 1887 ron Lehrer Fr. Basticr, hicr.

\section{Vipera latastei Boscá.}

Boulenger, Cat. III pag. 484; Boettger, Abh. Senck. Nat. Ges. Bd. 13, 1883 pag. 106.

9484 a. Erw. Iragonien, Nord-Spanien. Gesch. 1886 ron Ingenieur Carl Reuleaux, Mü̈nchen.

9484 b. Erw. und halbw. Tanger. Marokko. Gesch. 1881 ron Hans Simon, Stuttgart und Dr. merl. W. Kobelt, Schwanheim (Main).

\section{Vipera ammodytes (L.).}

B o ulenger, Cat. III pag. 485; Boettger, Sitz.-Ber. Berlin. Akad. Wiss. 1888 pag. 179.

9485̃ a. 2 erw. Prevesa, Epirus. Gesch. 1889-90 ron César C'onéménos, daselbst.

9485 b. Erw. Serbien. Gesch. 1890 ron Prof. Dr. Friedrich Kinkelin, hier.

9485 c. Erw. Orsova im Banat. Get. 1888 ron Prof. Dr. Angust ron Mojsisorics, Graz.

9485d. Erw. Grenze ron Kïrnten und Steiermark. Get. 1889 von demselben.

9485 e. 2 erw., 1 jung. Xenochóri, Nord-Euböa. Gesch. 1891 ron Fr. de Mimont, daselbst.

9485 f. Halbw. Siid-Tirol. Gesch. 1882 ron Prof. Dr. Ach. Andreae, Hildesheim.

9485 g. 3 erw. (Riippell, Cat. III FF 3 a-c). Dalmatien. Get. 1836 ron Michahelles, Mïuchen. 
7. Vipera lebetina (L.).

Boulenger, Cat. III pag. 487; Boettger, Ber. Senck. Nat. Ges. 1880 pag. 267 (enphratica), Abl. Senck. Nat. Ges. Bd. 13, 1883 pag. 105 (enthratica var. munitanica). Zool. Jalrrb. Bd. 3, 1888 pag. 946 (obtusa), Sitz. - Ber. Berlin. Akad. 1888 pag. 180 und Zool. Jahrb. Bd. 3, 1888 pag. 947 (xanthina) und Zeitschr. f. dl. ges. Naturw. Bd. 49, 1877 pag. 288.

9487 a. 2 halbw. (var. xanthina Gray). Haiffa, Nyrien. Gesch. 1884 von Hans Simon, Stuttgart.

9487 b. Erw. (var. enphratica Hart.). Kopet-dagh, Transkaspien. Gesch. 1890 von C. Eylandt, Askhabar.

9487 c. Erw. (var. manitanica Guich.). Tanger, Marokko. Erh. 1881 von der IV. Rüppellreise Dr. med. W. Kobelt's, Schwanheim (Main).

9487 d. Kopf (var. euphratica Mart.). Chodsha-kala, Transkaspien. Gesch. 1888 ron Staatsrat Dr. Gustav von Radde, Excz., Tiflis.

9487 e. Erw. (var. euphratica Mart.). Beirut, Norl-syrien. Gek. 1880 ron Yaturalienhändler Wilhelm schliiter, Halle (Saale).

\section{Vipera russelli (Shaw).}

Boulenger, Cat. III pag. 490.

9490 a. Erw. Madras. Gesch. 1889 von Theorlor Kolb, daselbst.

9490 b. Jung. Kandy, Ceylon. Gesch. 1890 ron Dr. Adolf Strubell, Bonn. 9490 c. Jung. Ceylon. Gesch. 1846 von H. Worms, hier.

9490 l-e. 2 erw. (Gestopft). Ostindien. Gek. 1846 von Boissoneau.

\section{Genus III. Bitis Gray.}

1. Bitis arietans (Merr.).

B oulenger, Cat. III pag. 493; Boettger, Abh. Senck. Nat. Ges. Bit. 9, $187 t$ pag. 163, Ber. Senck. Nat. Ges. 1888 pag. 89 und Zool. Anzeiger 1893 pag. 130.

9493 a. Erw. 11. jung. Banana, L'uter-Congo. Gek. 1887 von Paul Hesse, Venelig. 9493 b. Kopf. Landschaft Sus. Siidl-Marokko. Gesch, 1872 von den Proff.

Drr. Freih. ('. ron Fritsch, Halle (Saale), und J. .J. Rein, Bonn.

9493 c. Erw. Banana, Unter-Congo. Gek. $188 j$ ron Paul Hesse.

9493 d. Erw. Kapland. Gesch. 1873 von Dr. Löwenthal, hier.

9493 e-f. 2 erw. (Gestopit). Kordofin. (Kiippell, (at. IIl FE 2 a-b als

Vipera). Gesch. 1829 von Dr. med. Eduard Riippell, hier.

\section{Bitis cornuta (Daud.).}

Bonlenger, C'at. III pag. 497; Boettger, Ber. Senck. Nat. Ges. 1887 pag. 168 (Viperel).

9497 a. Erw. Damaraland. Gesch. 1887 von Prof. Dr. Hans Schinz. Kürich. 9497 b. Erw. (Gestopft). Kapland. Alte Sammlung 1845. 


\section{Bitis callalis (Smith).}

Bouleuger, Cat. III pag. 498; Boettger. Ber. Senck. Nat. Ges. 1886 pag. 6, 1887 pag. 167 (Vipera) und 1886 pag. 8, Taf. 1, Fig. 1 (Vipera schneideri).

9498 a. Erw. ․ Namalant. Gesch. 1876 ron Oberlehrer Müller, hier.

9498 b. Erw. ठ. Angra Pequena, Deutsch-Siidwestafrika. Gesch. 1886 von Prof. Dr. Oskar Schneider, Dresden.

9498 c. Erw. ․ Route Angra Pequena-IÁns, frofs-Namalaud. Gesch. 1887 von Prof. Dr. Hans Schinz, Zürich.

9498 d. Erw. + (Original von Vipera sehneideri Bttgr.). Angra Pequena. Grof-Namaland. Gesch. 1886 ron Prof. Dr. Oskar Schneider.

\section{Bitis gabonica (D. B.).}

Boulenger, Cat. III pag. 499.

9499 a. Kopf. Odumase, Ost. Goillkiiste. Giesch. 1892 ron Hans Simon. Stuttgart.

9499 b. Erw. IIundame, Kamerum. Gek. 1897 ron Dr. Georg Kraatz, Berlin.

\section{Bitis uasicolnis (Shaw).}

Boulenger, Cat. III pag. j00.

9500 a. Erw. Liberia, West-Afrika. Gek. 1891 von Gustar Schneirler. Basel. 9500 b. Halbw. Kamerun. Gek. 1897 von Dr. Georg Kraatz, Berlin.

\section{Genus IV. Cerastes Igl.}

\section{Cerastes coruutus (L.).}

Boulenger, Cat. III pag. 502; Boettger, Ber. Senck. Nat. Ges. 1880-8I pag. 145 (Vipera cercastes).

9502 a. Erw. ․ (Rïppell, ('at. III FF 1 d als Vipera eerastes). İypten. Gesch. 1828 ron Dr. med. Eduard Rïppell, hier.

9502 b. Erw. Wiiste von Tunis. Gesch. 1893 von Baron C. von Erlanger. Nieder-Ingelheim.

9502 c. Erw. und halbw. Gèryville, Algeriscle salıara. Erh. 1881 ron der IV. Rüppellreise Dr. med. W. Kobelt's. Schwanheim (Main).

9502 d. 2 erw. Biscra, Agerische Sahara. Gek. 1885 ron Lonis Fischer, daselbst.

9502 e. 3 erw. (Rïppell, Cat. III FF 1 a-c als V. cerastes). İypten. Gesch. 1828 von Dr. mel. Eduard Rüppell, hier.

9502 f. Kopf. Ebeutaher. Gesch. 1896 ron der Nenen Zoolog. Gesellschaft, hier.

9502 g. Erw. (Gestopft). El)endaher (Rïppell, Cat. III FF 1 b als V.cercustes). Gesch. 1828 von Dr. med. Elluard Riippell. 
2. Cerastes vipera (L.).

B 0 ulenger', Cat. III pag. 503.

9503 a. Erw. Siid-Tunis. Gek. 189 jon Paul Spatz, Gabes.

9503 b. Erw. \& (Rüppell. Cat. III FF 1 e als Vipera cerastes). İgyteu. Gesch. 1828 von Dr. mert. Eduard Rïppell, hier.

9503 c. Halbw. Biscra, Prov. Constantine, Mgerien. Gek. 1885 von Louis Fischer, daselbst.

9503 ז. Erw. Siid-Tunis. Gesch. 1896 von der Neuen Zoolog. Gesellschaft, hier.

9503 e. Erw. Ebendaher. Gesch. 1896 von derselben.

\section{Genus V. Echis Merr.}

1. Echis carinatus (Schnd.).

B oulenger, Cat. III pag. 505; Reuß, IIus. Senck. Bu. 1, 1834 pag. 157 (pavo) und pag. 160, Taf. 7, Fig. 2 (caria); Boettger, Zool. Jahrb. Bd. 3, 1888 pag. 949 (arenicola).

9505̆ a. Erw. (Riippell, Cat. III JJ 1 a als caria). Nubien. Gesch. 1828 von Dr. med. Eduard Riippell, hier.

9505 b. Erw. Duschak, Transkaspien. Gesclı. 1889 von Staatsrat Dr. Gustar von Radde, Excz., Tiflis.

9505 c. Halbw. Madras. Gesch. 1889 von Theodor Kolb, daselbst.

9505 d. Halbw. Bombay. Gesch. 1887 von Prof. Dr. Ferdinand Richters, hier. 950弓̆ e. 3 erw. İgypten. (Rïppell, Cat. III JJ $1 \mathrm{~b}-\mathrm{d}$ als paro). Gesch. 1828 von Dr. med. Eduard Rïippell.

\section{Genus VI. Atheris Cope.}

1. Atheris squamiger (Hallow.).

Boulenger, ('at. III pag. 509; Boettger, Ber. Senck. Nat. Ges. 1888 pag. 90 (squamigera), Zool. Anzeiger 1887 pag. 6501 und Ber. Senck. Nat. Ges. 1888 pag. 92, Taf. 2, Fig. 7 (laericeps).

9509 a. 2 erw. (Originale von A. lueviceps Bttgr.). Banana, Unter-Congo. Gek. 1887 von Panl Hesse, Venedig.

9509 J. 3 Ste. IIundame, Kaneruu. Gek. 1897 von Dr. Georg Kraatz, Berlin.

2. Atheris ceratophorus Weru.

Bonlenger, Cat. III pag. õ10.

9510 a. Erw. Buloa bei Tanga, Deutscli-(Ostafrika. Get. 1896 rom Naturl. Inseum, Berlin. 


\section{Genus VII. Atractaspis Smith.}

1. Atractaspis hildebrandti Pts.

Bonlenger, Cat. III pag. 512.

9512 a. Erw. Peccetoni, Witulandl, Ost-Afrika. Gesch. 1897 von Dr. Alfred Voeltzkow, Berlin.

2. Atractaspis congica Pts.

B o ul enger, Cat. III pag. 513; B o ettger, Ber. Senck. Nat. Ges. 1888 pag. 87 (irregularis var.).

9513 a. Erw. Povo Netouna bei Banana, Unter-Congo. Gek. 1887 von Paul Hesse, Venedig.

3. Atractaspis rostrata Gthr.

B o u lenger, Cat. III pag. 5́14.

9514,1 a. Erw. Boroma am Sambesi. Gescl. 1895̃ ron Prof. Dr. O. Boettger, hier.

4. Atractaspis bibroni Smith.

Boulenger, Cat. III pag. 515; B oettger, Ber. Senck. Nat. Ges. 1887 pag. 16 (irregularis var.).

9515a. Erw. Route | Aus-Angra Pequena, Grofs- Mammaland. Gesch. 1887 rou Prof. Dr. Hans Schinz, Ziirich.

\section{Subfamilie 2. Crotalinae.}

Genus I. Ancistrodon Palis.

1. Allcistrodon piscivorus (Lacép.).

Boulenger, Cat. III pag. 520.

9520 a. Erw. Texas. Gek. 1877 von Hamburg.

2. Ancistrodon bilineatus Gthr.

Bonlenger, Cat. III pag. כ̌21.

9521 a. Erw. Retalhuleu, Guatemala. (iesch. 1894 von Carl Fleischmann, Guatemala.

3. Ancistrodon contortrix (L.).

B oul eng e r. Cat. III pag. 522.

95222 a. Erw. Verein. Staaten. Gescl. 1896 ron der Nenen Zoolog. Gesellschaft, hier.

952: b. Erw. Ebendaher. Gesch. 1877 von derselben. 
4. Ancistrodon halys (Pall.).

Boulenger, (at. III pag. 524; Boettger in Radde's Faun. Flor. (aspi-Gieb. 1886 pag. 74 (Trigonocephalus).

9524,1a. Erw. Lyrik bei Lenkoran im Talyschgebiet, Transkaukasien. Gesch. 1886 von Hans Leder, Jauernig.

\section{Ancistrodon blomhoffi (Boie).}

Boulenger, Cat. III pag. 525 .

9525 a. Halbw. Yokohama, Japan. Get. 1889 von Bernhard Schmacker, Shanghai.

9525 b. 3 erw. Nippon. Gek. 1886 auf der Dr. Ahlberg'schen Auktion, hier. 95̃25 c. Erw. Insel Jeso, Nord-Japan. Get. 1889 von Bernl. Schmacker.

9525 d. Erw. Nippon. Gesch. 1876 ron Prof. Dr. J. J. Rein, Bonn.

9525 e. 2 erw. (Riippell, Cat. III BB $6 \mathrm{a}-\mathrm{b}$ als Trigonocephalus). Japan. Get. 1838 rom Nat. Rijksmuseum in Leiden.

6. Ancistrodon rhodostoma (Boie).

Boulenger, Cat. III pag. 527; Boettger, Ber. Offenbach. Ver. f. Naturk. 1892 pag. 135.

9527 a. Erw. Reich Jolo auf Javil. Gescll. 1868 von E. Lörsch, hier.

9527 b. Erw. (Riippell, Cat. III BB 7 a als Trigonoceplualus). Javil. Get. 1838 vom Naturh. Lijkssmuseun in Leiden.

9527 c. 2 erw., 2 jung. Ungebung ron Buitenzorg, West-Java. Gesch. 1890 vou Dr. Adolf Strubell, Bonn.

7. Ancistrodon hypule (Merr.).

Boulenger, Cat. III pag. 528.

9528 a. 6 Ste. Ceylon. Gesch. 1846 von H. WVorms, hier.

\section{Genus II. Lachesis Daud.}

1. Lachesis muta (L.).

Boulenger, Cat. III pag. 534 .

9534 a. Erw. Im Innem der Insel Trinidad. Gesch. 1890 von Fr. W. Lrich und R. R. Mole, Port of Spain.

9533 b. Erw. (gestopft). Brasilien (Riippell, Cat. III CC 1 a als rombeuta). Gesch. 1840 von Baron A. von Rothschild durch Prof. Freyreib.

2. Lachesis lanceolata (Lacép.).

B oulenger, Cat. III pag. 535 .

953วัa. 2 elw. (Riippell, Cat. III BB $2 * a-b$ als Trigonoeeplutus atrox).

Brasilien. Get. 1838 rom Grafen Jenison, Heidelberg. 
9535 b. Halbw. Peru. Gesch. 1884 ron Wilhelm Scheuermann, hier.

9535 c. Halbw. São Paulo, Brasilien. Gek. 1876 ron Prof. Cárlos Miiller, daselbst.

9535 d. 2 erw. Brasilien. Gek. 1887 von C. A. Pöhl, Hamburg.

9535 e. Erw. u. 2 halbw. (Rüppell, Cat. III BB 2 a-c als Tr.atrox). Brasilien. Get. 1838 rom Grafen Jenison.

9535 f. Kopf. Trinilad. Gesch. 1893 ron Fr. W. Urich und R. R. Mole, Port of Spain.

9535 g. 2 erw. (Riippell, Cat. III BB $1 \mathrm{a}-\mathrm{b}$ als Tr.jararaca). Itheos, Brasilien. Gesch. 1838 von Gebr. Koch. hier.

953 h. Halbw. и. 5 Junge. Carácas, Veueznela. Gek. 1897 von Ingenieur Müller, hier.

$$
\text { 3. Lachesis atrox (L.). }
$$

B oulenger, Cat. III pag. 537.

9537 a. Halbw. Nicaragua. Gesch. 1892 von Prof. Dr. Oskar Boettger, hier. 9537 b. Halbw. San José, Costa Riea. Gesch. 1893 ron Carl Fleischmann. Guatemala.

\section{Lachesis picta 'T'schudi.}

B oulenger, Cat. III pag. 540; Jan, Icon. Oph. Lief. 47, Taf. 3, Fig. 3 (Bothrops).

$95+(1,1$ a. Erw. (Varietät mit meist ungeteilten Subcandalen). Carácas, Venezuela. Gek. 1897 von Ingenieur Miiller, hier.

$$
\text { 5. Lachesis altelinata (D. B.). }
$$

B oulenger, Cat. III pag. 541 .

95̃41 a. Erw. Rio Grande do Sul, Süd-Brasilien. Get. 1888 ron Prof. Dr. $\mathrm{H}$. ron Ihering, daselbst.

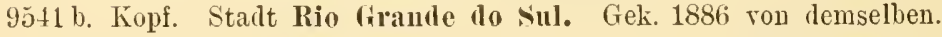

$$
\text { 6. Lachesis neuwiedi (Wgl.). }
$$

Boulenger, Cat. III pag. 542; Boettger, Zeitschr. f. 1. ges.

Naturw. Bd. 58, 1885 pag. 239 (Bothrops diporus).

9542 a. Kopf u. halbw. Stadt Rio Grande do Sul, Süıl-Brasilien. Gek. 1886 von Prof. Ur. H. ron Ihering, daselbst.

$95+2$ b. Erw. Paraguily. Get. 1885 rom Naturhist. Institut Linnaea, Berlin. 95 t2 c. Halbw. Rio (Hrande do sul. Get. 1888 von Prof. Dr. H. ron Thering.

\section{Lachesis num ifera (Riipp.).}

B ou lenger, Cat. III pag. 54t; R ï p pe 11, Verz. Senck. Mns. Amphib. 1845 pag. 21 (Atropos).

9544,1 a. Halbw. (Original. Mexiko (lïippell, (at. 111 kik 1 a). Gesch. 1810 ron Dr. med. Eluard Rüppell, hier. 
8. Lachesis brachystoma (Cope).

Boulenger, Cat. III pag. 547.

9547 a. Erw. u. 2 Junge. Cairo (La Junta) bei Limon, Östliches Costa Rica. Gesclı. 1893 von Carl Fleischmann, Guatemala.

9. Lachesis monticola (Gthr.).

Boulenger, Cat. III pag. 548 .

9548 a. Erw. und jung. Darjeeling, Ost-Hinalaya. Get. 1891 vom Britislı IIuseum (N. H.), London.

10. Lachesis strigata (Gray).

B onlenger, Cat. III pag. 549 .

9549,1 a. 2 erw. Nilgiris, Niid-Indien. Gesch. 1890 vom Naturh. Inseum in Madras.

11. Lachesis flavoviridis (Hallow.).

B o u lenger, Cat. III pag. 550 .

9550 a. Halhw. Insel O-shima, Linkiu-(iruppe. Gesch. 1889 von Major H. von Schönfellt, Offenbach (Main).

\section{Lachesis lutea (Bttgr.).}

B oulenger, Cat. III pag. 553; B oettger, Ber. Offenbach. Ver. f. Naturk. 1895 pag. 111 (Trimeresurus).

95553 a. 2 erw. (Originalexemplare). Yaeyama auf Mijakoshima, siidliche Liukiu-Inseln. Gesch. 1895 von Bernhard Schmacker, Shanghai.

\section{Lachesis graminea (Shaw).}

B o u lenger, Cat. III pag. 5̌́t; Boettger, Ber. Senck. Nat. Ges. 1887 pag. 50 und 1894 pag. 135 (Trimeresurus).

955̃ a. Halhw. Bangkok, Sianı. Gesch. 1885 von Konsul Dr. O. Fr. von Ioellendorff, Manila.

95்̃ b. Halbw. Java. Gesch. 1886 von Oberlehrer J. Blum, hier.

9554 c. Erw, Siid-Formosa. Get. 1888 von Bernhard Schmacker, Shangliai. 955் d. Erw. (Rïppell, (at. III BB 4 a als Trigonocephalus viridis). Insel Timor. Get, 1838 rom Rijksmuseum in Leirten.

9554 e. Jung. Samarang auf Java. Gesch. 1871 von Konsul Eilward Jacobson, hier.

$9 \check{\partial} 4$ f. 2 erw. Insel Hainan, Süı-China. Gesch. 1894 von Bernh. Schmacker. 955 g. Erw. u. jung. Ebendaher. Gek. 1885 von Otto Herz, St.-Petersburg. 
14. Lachesis flavomaculata (Gray).

Boulenger, Cat. III pag. 5̄56, Taf. 25, Fig. 3.

9550 a. 4 erw., 1 halbw. Mlajayjay, Prov. Laguna, Lnzon. Gesch. 1890 von

Konsul Dr. O. Fr. ron Hoellendorff, Manila.

$95 \check{5} 6$ b. Erw. Plıilippinen. Gesch. 1891 von demselben.

95う6 c. 3 erw., 1 halbw., 4 jung. Jlajayjay, Pror. Laguna, Luzou. Gesch. 1891 von demselben.

15. Lachesis allamallensis (Gthr.).

Boulenger, Cat. III pag. 558; B oettger, Ber. Offenbach. Ver. f. Natmk. 1892 pag. 92 (Trimeresurus).

9558 a. Erw. u. halbw. Nilgíris, Siid-Indien. Gesch. vom Naturh. Inuseum in IIadras.

16. Lachesis trigonocephala (Daud.).

B oulenger. Cat. III pag. 55̃9; Boettger, Ber. Offeubach. Ver. f.

Naturk. 1892 pag. 93 (Trimeresurus).

9559 a. Halbw. Ceylon. Gek. 1889 ron Hans Fruhstorfer, Berlin.

9559 b-c. 5 erw., halbw. n. jung. Ebendalıer. Gesch. 1846 von H. Worms, hier.

17. Lachesis macrolepis (Bedd.).

B oulenger. Cat. III pag. 560; B oettger, Ber. Offenbach. Ver. f.

Naturk. 1892 pag. 93 (Trimeresurus).

9560a. 2 erw. Kodaikanal, Palni Hills, Niid-Iıdien. Gesch. 1889 von Theodor Kolb, Jadras.

18. Lachesis pulicea (Buie).

B oulenger, Cat. III pag. 560; Bocttger, Ber. Offenbach. Ver. f.

Naturk. 1892 pag. 136 (Trimeresurus).

9560,1 a. Jung. Buitenzorg, West-Java. Gesclı, 1890 von Dr. Adolf Strubell, Bonn.

9560.1 b. Erw. (Rüppell, Cat. III BB 5a als Trigonocepllulus). Java. Gesch. 1838 von Dr. W. von Siebold, Leiden.

19. Lachesis borlleensis (Pts.).

B oulenger, Cat. III pag. 561 .

95̋1 a. Erw. u. jung. Baramfluß in Nord-Borneo. Erh. 1895 von der Rï̈ppellreise Prof. Dr. WV. Kiikentlials, Jena. 
20. Lachesis wagleri (Boie).

Boulenger, Cat. III pag. 562.

9562 a. Jung (Typus). Baramfluß in Nord-Borneo. Erh. 1895 von der Rüppellreise Prof. Dr. W. Küikenthals, Jena.

9562 b. Halbw. (Typus). Bindjey, Sultanat Deli, Nordost-Smmatra. Gesch. 1890 von Theodor und Eghert Engelharl, daselbst.

9562 c. Halbw. (Typus). Deli, Nordost-Nimatra. Gesch. 1886 von ('lemens Holwiesner, hier.

9562 d. Halbw. (Typus). Insel Hindanao, Pbilippinen. Gesch. 1890 von Konsul Dr. O. Fr, von Moellendorff, Nanila.

9562 e. Erw. (Typus). Mittel-Luzou. Gesch. 1890 von demselben.

9502 f. Halbw. (Typus). Insel Leyte, Philippinen. Gesch. 1893 von demselben. 9562 g. Erw. (Typus). Pontianak, West-Borneo. Gesch. 1893 von Dr. H. Lenz, Lübeck.

9502 h. Erw. (var. D). Deli, Nordost-Sumatra. Gesch. 1886 von Clemens Hohwiesner.

\section{Lachesis bilineata (Wied).}

Boulenger, Cat. III pag. 565; Jan, Icon. Oph. Lief. 47, Taf. 1, Fig. 2 (Bothrops).

9อ̃6ว a. Halbw. (Rïppell, Cat. III BB 3 a als Trigonocephlulus; Original zu Jan's oben citierter Abbillung). Brasilien. Gesch. 1836 von Schïff Dr. Carl von Heyilen, hier.

\section{Lachesis schlegeli (Berth.).}

Boulenger, Cat. III pag. 567.

9567 a. Erw. Cuenca, Ecualor. Gesch. $1896^{\circ}$ von Konsul F. C. Lehmann, Popayán.

9567 b. 2 halbw. Cairo (La Junta) bei Limon, Oestliches Costa Rica. Giesch. 1892 von Carl Fleischmann, Guatemala.

\section{Genus III. Sistrums Garm.}

1. Sistrulus catenatus (Rafin.).

Boulenger, Cat. III pag. 570.

9570 a. Erw. Milwankee, Wisconsin. Gesch. 1893 von Bierbrauer R. Henrich, hier.

9570 b. Erw. (Rüppell, Cat. III DD 3 a als Crotalus miliaris). Verein.Staaten. Gesch. 1838 von Dr. med. Reuß und Engelmann, St. Louis. 


\section{Gemus IV. Crotalus L.}

1. Crotalus terrificus (Laur.).

Boulenger, Cat. III pag. ว̌73.

9573 a. Erw. (var. A). Mexiko (Rüppell, Cat. III DD 1 b als horridus). Gesch. 1832 von $\mathrm{F}$. A. Dillenburger, daselbst.

9573 b. 3 Klappern, 4 Giftzähne. Rio Grande do sul, Süd-Brasilien. Gesch. 1875 von Oberlehrer Dr. Finger, hier.

9573 c. Erw. (var. B). Brasilien (Riippell, Cat. III DD 1 a als horridus). Get. 1840 rom Naturh. Hofmuseum, Wien.

9573 d. Erw. u. jung (var. B). Carácas. Venezuela. Gek. 1897 von Ingenieur Jiiller, hier.

2. Crotalus durissus L.

Boulenger, Cat. III pag. 578.

9578a. Erw. (Gestopft). SiidlostI. Verein. Staten (Rüppell, C'at. III DD 2a). Gek. $184 t$ vou Cardua.

3. Crotalus horridus L.

Boulenger, Cat. III pag. 578.

9579 a. Erw. Yerein. Staaten. Gesch. 1866 ron der Neuen Zoolog. Gesellschaft, hier.

9579 b. 2 erw. Ebendaher. Gesch. 1892 ron derselben. 


\section{$143-$ \\ Yerzeichninis der Schenker.}

Herr Ahlburg, Prof. Dr.. Tokyo (Tapan) $†$.

"Albrecht, Missionar, Honore (Ostindien).

"Andreae, Prof. Dr. Ach., Direktor des Römer-Nuseums, Hillesheim.

Augsburg, Huseum des Naturhistorischen Vereins.

Herr B a m berger. Мax, Kaufmann, Frankfurt a. M.

Bas el, Naturhistoriscles IIuseum.

Herr Bastier, Fr.. Lehrer, Frankfurt a. II.

Herren Bauditsch \& ('o., Naturalienhündler. Triest.

Herr Becker, Adam, Sydney (Neusiidwales) $\dagger$.

von Bedriaga, Ir. phil. Jacques, Zoologe, Nizza.

, Behrends, Phil. Friedr., Frankfurt a. M.

" Benecke, Dr. med. O., Deli (Sumatra).

"B erg, Dr. phil. Cárlos, Direktor des Naturhistorischen Museums, Buenos Aires.

Berlin. Naturhistorisches Institut Linnaea.

Kgl IIuseum fïr Naturkunde.

Herr Bernus, W. A., Bankier, Frankfurt a. גI. †.

"Beyerbach jun., Frankfurt a. II.

"Beyschlag, Fritz, Kaufmann, Deli (Sumatra).

, Bl um, Oberlehrer J., Frankfurt a. M.

" B oettger, Prof. Dr. phil. Oskar, Frankfurt a. M.

" Boettger, Prof. Dr. Ruc. Christ., Frankfurt a. M. †.

"Boissoneau.

"Boulenger, G. A., F. R. S., Sektionschef a. British IIusenm (Nat. Hist.),

London.

* Brau er, Dr. phil. Angust, Privatdocent für Zoologie, Marburg (Hessen).

" Braun, Dr. phil. Max, Prof. der Zoologie, Rostock.

" Braun, Feldmarschall Philipp, Lima (Per'u).

"Broemme, Dr. phil. Christian, Chemiker, Wiesbaden $\dagger$.

"B ïtschli, Hofrat Dr. phil. Otto, Prof. der Zoologie, Heidelberg.

"Cardua (viell. Dr. Carove, Friedberg) $†$.

- Conéménos, César. Prevesa (Epirus).

"Cordier, K. Will.. Hofrat, Frankfurt a. M. $\dagger$.

D a rmstadt, Großherzgl. Naturhistorisches Iuseum.

Herr De ermann, H.. Menageriebesitzer, Frankfurt a. M.

"Dietze, Karl, Kunstmaler. Jugenheim (Bergstrasse).

"Dillenburger, F. A., Kanfmann, Jexiko $†$. 
Herr Döbel, Dr. med. H. L.. Bataria (Java) †.

Duschanek, Josef, São Paulo (Brasilien).

" Ebenau, Karl, Konsul, Kaufmann, Hamburg.

" E dinger, Prof. Dr. merl. Ludwig. Frankfurt a. M.

n Eich, Bergingenieur, Geisnidla (Oberhessen) $\dagger$.

" Emm el, Ferdinand, Kanfmann, Hamburg.

"Emmel. Friedrich, Arequipa (Períi).

Herren Engel hard. Febr. Theodor und Egbert, Deli (Sumatra).

Herr Engelmann, Dr. merl. Johann Georg, St. Louis (Miss.) †. von Erlanger, Baron Carlo, Nieder-Ingellreim.

n Eylanilt, C., Naturalist, Askhabal (Transkaspien) $\dagger$.

" Fing er, Dr. phil., Oberlehrer, Frankfurt a. M. $\dagger$.

" Finckh, H., Ludwigsburg $†$.

» von Fischer, Johann, Zoologe, Jontpellier (Hérault).

" F is cher, Louis, Biskra (Algerien).

„Flach, Dr. med. Karl, Aschaffenburg.

» Fleck, Dr. phil. Eduard, Azuga (Rumänien).

" Fle ischmann, Karl. Kaufmann, Guatemala.

"Flins ch, Heinrich, Frankfurt a. JI.

Frankfurt a. M., Nene Zoologische Gesellschaft.

Herr Freyrei $B$, Georg Wilhelm, Prof. der Zoologie, Rio de Janeiro $†$.

" von Fritsch, Freiherr Dr. Karl, Prof. rler Mineralogie, Halle a. Saale.

n Frommel, August, Frankfurt a. II.

"Frnhstorfer, Hans, Naturalienhändler, Berljn.

"Gerold, (r., Ingenieur, Port of Spain (Trinilad).

n Geyler, Dr. phil. Theodor, Direktor des Botanischen Gartens, Frankfurt a. M. $\dagger$.

Go I f $\mathrm{f} B$, Otto, Zoologe, Halle a. S.

" Ha a s e, Dr. phil. Erich, Direktor des Naturhistor. Museums, Bangkok (Siam) $\dagger$.

$\mathrm{Hamburg}$, Naturhistorisches Museum.

Herr Han a u, Dr. merl. Arthur, St. Gallen.

H a p p e, Karl, Rödelheim.

Hen ricli, Karl, Braner, Frankfurt a. II.

Hen rich, Rudolf, Brauer, Frankfurt a. II.

Herath, Gustav, Frankfurt a. II.

" Her'z, Otto, Naturalist, St. Petersburg.

" Hesse, Paul, Kaufmann, Venedig.

n von H e yden, Schöff Dr. Karl, Frankfurt a. Mr. †.

n von Heyden, Major z. D. Dr. phil. Lukas, Frankfurt a. M.

"Hirsch, Karl, Kanfmann, Frankfurt a. M.

"Hohwiesner, Clemens, Kaufmann, Deli (Sumatra).

Hollmann, Lehrer, Vegesack bei Bremen.

" Hï buer, i. F. Hübner \& Schlesinger, Kaufmann, Leipzig $\dagger$.

Hï bner, Georg, Naturalist, Dresden.

» von Ihering, Dr. phil. Hermann, Direktor des Naturhist. MInsenuns, Sào Paulo (Brasilien). 
Herr J a cobson, Edwarl, Konsul, Frankfurt a. II. †.

” Jas s o y, Dr, phil. August, Apotheker, Frankfurt a. II.

ᄁ Jenissou, Graf, Heidelberg $†$.

"Jickeli, Dr. phil. Karl Fr., Hermannstalt (Siebeubürgen).

K arlsru he, Zoolog. Institut der Grh. Technischen Hochschule.

Herr Keller, Dr. phil. Konrad, Prof. der Zoulogie, Zürich.

" Kinkelin, Apotheker Adolf, Nïrnberg.

K inkelin, Prof. Dr. Friedrich, Frankfurt a. II.

K irchner, Wilhelm, Deutscher Konsul, Sylney (Nensïrlwales) $\dagger$.

Kl 1 ïsi, Konrad, Zürich.

" Knobla uch, Ferdinand, Konsul in Neukaledonien, Frankfurt a. M. †.

"Kn obla uch, Karl, Kanfmann, Frankfurt a. MI.

" Kobelt, Dr. med. Wilhelm, Schwanheim a. II.

" K $0 \mathrm{ch}$, Adam, Konservator, Frankfurt a. I.

" K o ch III., G., Frankfurt a. II.

"Koch, Otto, Kaufmann, Cebú (Philippinen) $\dagger$.

Herren Koch, W. und Bruler, Brasilien $†$.

Herr König, Dr. phil. Alex., Prof. der Zoologie, Bonn.

» Kolb, Theodor. Kaufmann, Madras $\dagger$.

\# Koperberg, M., Mijningenienr, Pontianak (Borneo).

\# Kraatz, Dr. phil. Georg, Entomologe, Berlin.

Fran Kr ämer, H., Wwe., Frankfurt a. II.

„ Krieb, H., Wwe., Frankfurt a. II.

Herr K ükenthal, Dr. Willy, Prof. der Zoologie, Jena.

L e de r, Hans, Naturalist, Janernig (Oesterr.-Schlesien).

" Lehmaun, F. C., Deutscher Konsul, Popayán (T. S. Columbia).

Le i d e 1 , Kgl. Naturhist. Rijksmusenm.

Herr Le n z, Dr. phil. Heimr., Direktor des Naturhist. Museums, Lübeck.

" von Leuchtenberg, Herzog Max E. J. N., München $†$.

„ Lörsch, E.. Franlifurt a. M.

"Löwentha 1, Dr. phil., Frankfurt a. II. †.

Lond on, British Museum (Nat. Hist.).

Herr vou L u dwig, Baron, Stuttgart $\dagger$.

L ï beck, Naturlistorisches Musenm.

Herr Luin ing, Theodor, Apotheker, Ciudarl Bolivar (Venezuela).

Madras, Government Central Juseum. Egmore.

Herr II a u B, F., Kgl, Belgischer Konsul, Valencia (Venezuela).

» Ii celi, Francesco, Tunis.

\# Irichabelles, Dr. med., Triest.

" de Mimont, Fr., Gutsbesitzer, St. Jean bei Xenochóri auf Eubüa $\dagger$.

» ron Iloellendorff, Dr. phil. O. Fr., Konsul des Deutschen Reiclies, Kowno (RuBland).

von Hojsisovics, Dr. phil. August, Prof. der Zoologie, Graz $†$.

》 Jole, R. R., Port of Spain (Trinidal).

n M ïller, Dr. phil. August, Naturalienhändler, Berlin.

n Ir ïller, Prof. Cárlos, São Paulo (Brasilien).

» II ̈̈ller, Amtsrichter, Lauterbach (Oberhessen). 
Herr J[ ii l ler. Ingenieur. Frankfurt a. JI. II ï $l \mathrm{ler}$, Oberlehrer. Frankfurt a. II.

" Nabert, D. O. W.. Lianfmam. Frankfurt a. M.

- Tägele, G.. Pfarrer, Waltersweier bei Offenburg (Baclen).

" Nolte, Karl, Ingenieur, Frankfurt a. M.

N ïrnberg. Jiuseum der Naturhistorischen Gesellschaft.

Offenbach, Naturhistorisches Museum.

Fran ron Pan huijs. Frankfurt a. M. $†$.

Herr Parrot, J. C', Frankfurt a. II.

^ Passarant, Ph. G., Sanitätsrat Irr. med.. Frankfurt a. II. †.

* Passavaut, Theodur, Kanfmann. Frankfurt a. II. †.

"Pauli, Hofrat Dr. med., Frankfurt a. II. $†$.

Peitsch, Dr. med., Bataria (Java) $†$.

St. Petersburg: Naturhist. Mnsenm der Ksl. Akademie.

Herr Pöhl. C. A.. Naturalienhändler, Hamburg.

" von Radde, Wirkl. Staatsrat Dr. Gustar, Exc., Direktor des Kaukasischen Iruseums, Tiflis.

„ R e gel, Dr. Fritz. Prof. der Geographie. Jena.

. Reibis eh, Panl, Dresten-Plauen.

" Reichard. Philipp. Huspitahneister. Frankfurt a. II.

" Rein, Geh. Reg.-Rat Prof. Dr. J. J., Bomm.

» rou Reinach, Baron Albert. Frankfurt a. 1 .

» Reitter, Edmunil. Entomologe, Paskan (Mähren).

7 Retowski, Staatsrat Otto, Gymnasiallehrer. Theolosia (Krim).

„ Renleaux. Karl, Ingenieur, München.

\# ReuB, Dr. merl. Arlulf, st. Louis (Miss.) †.

- Richters, Prof. Ir. phil. Ferdinand. Frankfurt a. II.

ᄁ Rössing, IV.. Frankfurt a. II. †.

" Rosenbach. Wr.. Frankfurt a. II. $†$.

. Rosenberg, W. F. H., Naturalienhändler. London.

" von Rothsehild, Baron A. M., Frankfurt a. M. $\dagger$.

" R ̈̈ppell, Dr. med. Ednard. Frankfurt a. M. †.

* Schädle, Dr. med. H., Tanger (Jarokiko).

"Schauf, Oberlehrer Dr. phil. WV., Frankfurt a. II.

"Sclıenck, Dr. phil. A., Privatılozent für Geographie, Halle a. S.

" Sclue u ermann, Wilhelm, Kanfuann, Frankturt a. II.

"Sehinz, Dr. phil. Hans, Prof, der Botanik, Zürich.

"Schlesinger, i. F. Hübner \& Schlesinger, Kanfmann, Leipzig.

Schlïter, Wilhelm, Naturalienhändler, Halle a. S.

» Schmacker, Bernhard, Kaufmann, Shanghai $\dagger$.

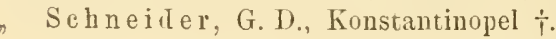

Schneider, Gustar, Naturalienhändler, Basel.

Schneider, Prof. Dr. phil. Oskar, Dresden.

"Schneiler, W., Frankfurt a. M. †.

"Von Schönfeldt, H., Oberstlientenant a. D., Siegen (Westfalen).

" von Schouler, W., Wiesbarlen $\dot{T}$.

n Schreiber, Dr. phil. Egid, schulrat, Görz. 
Herr vou Schrïter, Guido, Deutscher Konsul, San .Tosé (Costa Rica).

"Schumacher, Jakob, Frankfurt a. MI.

"Seitz, Albrecht, Kanfmann, Hamburg.

"Semon, Dr. phil. R. W., Prof. der Zoologie, Jena.

" de Seoane, Dr. V. Lopez, Jefe Superior de Administracion und Comisario Regio de Agricultura, Coruña (Spanien).

» Sichel, Dr. med. W., Frankfurt a. II. †.

"Siebert, August, Direlitor des Palmengartens, Frankfurt a. MI.

» vou Siebold, Dr. phil. Phil. Fr., Leiden †.

"Simon, Haus, Kaufmann, Stuttgart.

- Spatz, Paul, Gabes (Tunesien).

- Stiebel, Hofrat Dr. merl. Sal., Frankfurt a. MI. †.

* Stratz, Dr, med, Haag (Holland).

"Strubell, Dr, phil. Arolf, Privatdozent für Zoologie, Bonn.

"Stumpff, Anton, Kaufmann, Loucoubé (Nossibé) †.

n Stussiner, Josef, K. k. Postbeamter, Laibach.

, Textor, Albert, Eisenach.

Fräulein Thiesse, Joséphine, Chalkis (Euböa).

Herr Tschapeck, Hauptm.-Auditor Hippolyt, Wien $\dagger$.

"Urich, F.W., Kanfmann, Port of Spain (Trinilad).

nValentin, Dr. Jean, Geologe, Buenos Aires $\dagger$.

"Voeltzkow, Dr. phil. Alfred, Zoologe, Berlin.

"Weis, Albrecht, Bankbeanter, Frankfurt a. II

Werner, Dr. phil. Franz, Zoologe, Wien.

Wi chmann, Hermann, Kaufmann, Frankfurt a. II.

W i en, K. k. Zoologisches Hofmuseum.

Herr Will, Premierlientenant Dr. phil. Fr., Zoologe, Erlangen.

"Winter, Siegmund, Lithograph. Frankfurt a. M.

Herren Worms, Gebr. Gabriel \& Moritz. Colombo (Ceylon) $†$.

Herr Zipperlen, Dr. merl A., Cincinnati (Ohio). 


\section{Sachregister.}

Abastor, 79 .

Ablabes, 78.

Ablabophis, 35.

Acanthophis, 118.

Achalinns, 33.

Acrochordinae, 16.

Acrochordus, 16.

acuminatus (Dryophis), 108.

acuminatus (Oxybelis), 108.

acutirostris (Calamaria), 86

aegyptiaca (Dipsas), 91.

aeneus (Dryinus): 84.

Aepysurus, 114.

aesculapii (Colnber), 52.

aesculapii (Erythrolamprus), 109.

aestiva (Contia), 76.

aestivus (Herpetodryas), 76.

aestivus (Philorlryas), 99

affinis (Rladinaea), 67.

agamensis (Calamaria), 84.

agassizi (Contia), 99.

agassizi (Pseudablabes), 99.

agilis (Erythrolamprus), 109.

ahaetulla (Dendrophis), 60.

albifrons (Glauconia), 6.

albipuncta (Glauconia), 6.

albiventris (Colnber), 62 .

albiventris (Liophis), 61.

albocinctus (Simotes), 73

albofusca (Dipsas), 95.

albofusca (Leptodira), 95.

albus (Brachyorrhus), 32.

algirus (Zamenis), 45.

alkeni (Calamaria), 83.

almatensis (Liophis), 62.

alternans (Brachyorrhos), 87.

alternans (Eurostus), 87.

alternans (Hypsirhina), 87.

alternata (Lachesis), 138. amaliae (Coronella), 71. amaliae (Rhinechis'. 71.

Amblycephalidae, 127.

Amblycephalus, 127.

amethystinus (Python), 7.

ammodytes (Vipera), 132.

Amplorhinus, 99.

anamallensis (Lachesis), 140.

anamallensis (Trimeresurus), 140

Ancistrodon, 136.

angolensis (Dromophis), 104.

angolensis (Psammophis), 104.

anguilliformis (Aepysurus), 114.

angulata (Homalopsis), 30.

angulatus (Dipsadomorphus), 93.

angulatus (Helicops), 30.

annectens (Doliophis), 124.

annularis (Bungarus), 119.

annularis (Tropidonotus), 22, 23.

annulata (Dipsas', 95.

annulata (Leptodira), 95.

anomala (Rhadinaea), 66.

anomalus (Drepanodon), 72.

anomalus (Dromicus), 61.

anomalus (Oxyrrhopus), 72.

antareticus (Acanthophis), 118.

antillensis (Dromicus), 61.

Aparallactus, 111.

A poroplis, 65 .

Apostolepis, 111.

arctiventris (Calamaria), 77.

arenicola (Echis), 135.

arietans (Bitis), 133.

arietans (Vipera), 133.

arnensis (Simotes), 74 .

asianus (Zamenis), 43, 44.

asper (Engygrus), 11.

asperrimus ('Tropidonotus), 23.

Aspidites, 9. 
Aspirlura, 33 .

aspis (Vipera), 132.

assimilis (Apostolepis), 111.

ater (Coluber), 53.

ater (Tropidonotus), 21.

ater (Typhlops), 5.

Atheris, 135.

Atractaspis, 136.

Atractus, 79.

atratus (Streptophorus), 31.

atrox (Lachesis), 138.

atrox (Trigonocephalus), 137, 138.

aulicus (Dipsas), 37.

aulicus (Lycodon), 37.

auriculatus (Tropidonotus), 28.

aurora (Coronella), 35.

aurora (Lamprophis), 35.

australis (Enygrus), 11.

australis (Tropidonotus), 19.

austriaca (Coronella), 70.

badius (Atractus), 79. badius (Geophis), 79.

baliodirus (Ablabes), 78 .

batjanensis (Stegonotus), 39.

belli (Dispholidus), 108.

bennetti (Hypsirhina), 88.

bernieri (Dromicodryas), 17.

bernieri (Herpetollyas), 17.

berus (Vipera), 130.

bibroni (Atractaspis), 136.

bibroni (Enygrus), 11.

bicarinatus (Herpetodryas), 5 こ.

bicincta (Urotheca), 68.

bicolor (Coluber), 66.

bicolor (Glanconia), 6.

bicolor (Hydrophis), 112.

bifossatus (Drymobius), 47

bilineata (Calamaria), 8 ó.

bilineata (Hypsirhina), 88.

bilineata (Lachesis), 141.

bilineatus (Arleniophis), 124.

bilineatus (Aucistrodon), 136.

bilineatus (Bothrops), 141 .

bilineatus (Doliophis), 124.

bilineatus (Drymobius), 47.

bilineatus (Trigonocephalus), 141. bipunctatus (Coluber), 18.

bipunctatus (Erythrolamprus), 110.

bipunctatus (Tropidonotus), 18.

bisubocularis (Typhlops), $\check{.}$

bitaeniatus (Zamenis), 42.

Bitis, 133.

bitorquata (Alecto), 118.

bitorquata (Calamaria), $7 t$.

bitoryuatus (Hoplocephalus), 118

bitorquatus (Oligodon), 74.

bitorquatus (Oxyrrhopus), 96.

bivirgatus (Doliophis), 123.

bivittatus (Polyodontophis), 17.

blandingi (Dipsadomorphus), $9 \dot{f}$.

blandingi (Dipsas), 94.

blomheffi (Ancistrodon), 137.

blomhoffi (Trigonocephalus), 137.

blythi (Rhinophis), 15.

Boa, 12.

boa (Haplopeltura), 127.

boa (Nardoa), 7.

boddaerti (Drymobius), 47.

boddaerti (Herpetodryas), 47.

boettgeri (Callophis), 123.

boettgeri (Typhlops), 4.

bogorensis (Calamaria), 83.

Boidae, 7.

Boinae, 9.

bolivianus (Philodryas), 100.

Boodon, 35.

borneensis (Calamaria), 85.

borneensis (Lachesis), 140.

boulengeri (Elapechis), 119.

boulengeri (Elapsoidea), 119.

Brachyorrhus, 32.

brachystoma (Lachesis), 139.

braminus (Typhlops), 1.

breviceps (Rhadinaea), 65.

brevis (Silybura), 15.

brugmansi (Distira), 113.

buccata (Homalopsis), 88.

Bungarus, 119.

bungarus (Naja), 122 .

caeca (Naja), 121.

caerulescens (Hydrophis), 112.

Calamaria, 83. 
Calamorhabrlium, 82.

calligaster (Dendrophis), 56.

calligaster (Hemibungarus), 122.

callistus (Tropidonotus), 28.

Callopeltis, 97 .

Callophis, 123.

cana (Pseudaspis), 40.

candidus (Bungarus), 119.

caninus (Corallus), 11.

cantoris (Coluber), 50.

cantoris (Hydrophis), 113.

canus (Coluber), 40.

capense (Lycophidium), 36.

capensis (Bucephalus), 108.

capucinos (Lycodon), 37.

carbonarius (Zamenis), 43.

earinata (Dipsas), 128.

carinatus (Amblycephalus), 128.

carinatus (Echis), 135.

carinatus (Enygrus), 11.

carinatus (Herpetodryas), 55.

carinatus (Zaocys), 40.

carinicanda (Helicops), 30.

carinicauda (Homalopsis), 3 ).

easpius (Coluber), 43.

catenatus (Sistrurus), 141.

catesbyi (Dipsas), 129.

catesbyi (Leptognathus), 129.

catesbyi (Uromacer), 60.

caudalis (Bitis), 134.

caudalis (Vipera), 134.

caudolineatus (Dendrelaphis), 57.

Causus, 130.

Cemophora, 73.

cenchoa (Himantodes), 94.

cenchris (Epicrates), 9.

Cerastes, 134.

cerastes (Vipera), 134, 135.

cerastinus (Acanthophis), 118.

ceratophorus (Atheris), 135.

Cerberus, 88.

ceylonensis (Dipsadomorphus), 92.

ceylonensis (Haplocercus), 33.

ceylonicus (Bungarus), 119.

chamissoi (Dromicus), 60.

Chersydrus, 16.

childreni (Liasis), 7. chinensis (Hypsirhina), 88.

Chlorophis, 58 .

Chondropython. 9.

chrysargoides (Tropidonotus), 27.

chrysargus (Tropidonotus), 26, 27.

chrysochlora (Chrysopielea), 109.

Chrysopelea. 108.

citrina (Eteirodipsas), 90.

climacophorus (Coluber), 52.

cloelia (Osyrrhopus), 97.

cobella (Coronella), 50, 66.

cobella (Rhadinaea), 66.

coccinea (Cemophora), 73.

Coelopeltis, 101.

collaris (Calamaria), 84.

collaris (Contia), 76.

collaris (Coronella), 72.

collaris (Polyodontophis), 17.

Coluber, 49.

Colubridae, 16.

colubrina (Dijsas), 90.

culubrina (Eteirodipsas), 90.

Colubrinae, 16.

colubrinus (Platurus), 115.

colubrinus (Xenodon), 63.

congica (Atractaspis), 136.

congicus (Typhlops), 4.

conicus (Eryx), 13.

conjuncta (Glauconia), 6.

conspicillatus (Coluber), 52 .

conspicillatus (Tropidonotus), 21 .

constrictor (Boa), 12.

constrictor (Zamenis), 41.

contaminata (Calamaria), 8 j.

Contia, 76.

contortrix (Ancistrodon), 136.

cookei (Corallus), 10.

corais (Coluber), 49.

corallinus (Elaps), 12 .

Coralins, 10.

cornuta (Bitis). 133.

cornuta (Vipera), 133.

cornutus (C'erastes), 134.

coronata (Alecto), 117.

coronata (Denisonia), 117.

Coronella, 70.

coronella (Ablabes), it 


\section{$-151-$}

coronella (Contia), 77.

crassus ('Typhlops), 3.

cristagalli (Langaha), 90.

Crotalinae, 136.

Crotalus, 142 .

crucifer (Psammophis), 104.

cucullata (Coronella), 106.

cucullatus (Macroprotodon), 106.

cyanocineta (Distira), 113.

Cyclocorus, 35.

Cylindrophis, 14.

cynorlon (Dipsarlomorphus), 94.

cynodon (Dipsas). 94.

daemeli (Denisonia), 117.

dahli (Tyria), 44.

dahli (Zamenis), 44.

Dasypeltis, 86.

davisoni (Dryocalamus), 40.

davisoni (Ulupe), 40.

decemlineata (Contia), 76.

degenhardti (Stenorhina), 111.

dekayi (Isclnognathus), 31.

delalandei ('yphlops), 5.

Dendraspis, 127.

Dendrelaphis, 57 .

dendrophila (Dipsas), 92.

dendrophilus (Dipsarlomorphus), 92.

Dendrophis, 56 .

dendrophis (Drymobins), 48.

Denisonia, 117.

deppei (Colnber), 54 .

dhmmnarles (Zaocys), 40.

diadema (Lytorhynchus), 46.

diadema (Psendelays), 115.

diadema (Simotes), 46.

diadema (Zamenis), 46.

diarlematus (Streptophorus), 31.

Diemenia, 116.

digitalis (Coluber), 96.

digitalis (Oxyrrhopns), 96.

dimidiata (Calamaria), 83.

dinga (Typhlops), 5.

Dinodon, 38.

dione (Coluber), 51.

diporus (Bothrops), 138.

Dipsadomorphinae, 89.
I)ipsarlomorphns, 92.

dispar (Bryophis), 106.

Dispholirlus, 108.

dissolencus (Elaps), 126.

distanti (Glanconia), 6.

Distira, 113.

divinilogua (Boa), 12.

duliata (Coronella), 61, 62, 66 .

doliatus (Oxyrrhopus), 97.

dolichocercus (Tropidonotus), 25.

Doliophis, 123.

dorbignyi (Heterodon), 6 Ł.

dorbignyi (Lystrophis), 64.

dorsalis (Philothamnus), 59.

dorsatus (T'omodon), 99.

drapiezi (Dipsadomorphus), 93.

drapiezi (Dipsas), 93.

Drepanodon, 72.

Dromicodryas, 17.

Dromicus, 60.

Dromophis, 102.

Drymolius, 47.

Dryocalamus, 39.

Dryophis, 106.

dumerili (Stegonotus), 39.

durissus (C'rotalus), 142.

Echis, 135̄.

Elapechis, 118.

Elapinae, 115.

Elapoides: 33.

Elapomorphus, 111.

Elapops, 112.

Elaps, 125

elaps (Ophiophagus), 122.

elegans (Eryx), 13.

elegans (Himantodes). 94.

elegans (Philodryas), 101.

elegans (Psammophis), 104.

elegans (Tachymenis), 101.

emmeli (Atractus), 81.

emmeli (Geophis), 81.

Enhỵdina, 114.

Enhydris, 114.

enhydris (Hypsirhina), 87.

Enygius, 11.

Epicrates, 9. 
epinephelus (Liophis), 62. eques (Coluber), 18.

eques (Tropidonotus), 18.

elythrogrammus (Abastor), 79.

Erythrolamprus, 109.

erytlirurus (Coluber), 54.

Eryx, 13.

eschrichti (Typhlops), 4.

Eteirodipsas. 90.

Eunectes. 12.

euphratica (Vipera), 133.

enproctus (Typhlops). 1. 2.

entropis (Phrynonax), 49.

ereretti (Oligodon), 75.

eximia (Coronella), 72.

eydouxi (Aepysurus), 114.

fallax (Tarbophis), 91.

fasciata (Dasypeltis), 86.

fasciata (Naja), 121.

fasciatum (Lycophidium), 37.

fasciatus (Bungarus), 119.

fasciatus (Hamadryas), 122.

fasciatus (Hemibungarus), 122.

fasciatns (Hydrophis), 113.

fasciatus (Phrynonax), 49.

fasciatus (Tropidonotus), 24, 31 .

fasciolata (Dasypeltis), 86.

fasciolatus (Dryophis), 107.

favae (Atractus), 81.

favae (Calamaria), 81.

fedtschenkoi (Zamenis), 45.

ferox (Hypsirliynchus), 60.

ferox (Tropidonotus), 24.

fissidens (Erythrolamprns), 110.

fitzingeri (Coronella), 71.

fitzingeri (Oxyrrhopus), 97.

flagelliformis (Zamenis), 41.

flarescens (Coluber), 52.

flarescens (Tropidonotus), 23.

fla riceps (Bungarus), 120.

flaviceps (Doliophis), 123.

flaviceps (Macropisthodon), 28.

flavifrenatus (Aporophis), 65.

flavigularis (Thrasops), 59.

flaviventer (Typhlops), 3.

fla viventris (Coluber), $4 \mathbf{1}$. flaviventris (Zamenis), 41 .

flavolineatus (Herpetodryas), 55.

flaromaculata (Lachesis), 140.

flavoviridis (Lachesis), 139.

Fleischmannia, 69.

floriceps (Coluber), 45, 103.

florulentus (Coluber), 44, $\mathbf{4 5}$.

florulentus (Zamenis), 45.

Fordonia, 89.

formosanus (Dinodon), 38.

formosanus (Simotes), 73.

formosus (Dendrophis), 57.

formosus (Lycodon), 96.

forsteni (Rhabdophidium), 81 .

frontalis (Elaps), 126.

fronticinctus (Dryophis), 106.

fruhstorferi (Tetralepis), 35.

fuliginoides (Tropidonotus), 19.

fuliginosus (Boodon), 36.

fulvius (Elaps), 126.

furcatus (Elaps), 124.

Furina, 124.

fusca (Dipsas), 92.

fusca (Rhadinaea), 67.

fuscum (Trachischium), 32.

fuscus (Dipsadomor phus), 92.

fusens (Elapoides), 33.

fuscus (Epicrates), 10.

fuscus (Zaocys), 41.

gabonica (Bitis), 134.

gaimardi (Dipsas), 90.

Gastropyxis, 59.

gastrostictus (Elaps), 125.

geminatus (Polyodontophis), 17.

gemmiannulis (Hemibungarus), 123

gemonensis (Zamenis), 43.

Geodipsas, 89.

geometricus (Boodon), 35.

Geophis, 81.

gervaisi (Calamaria), 83.

getuia (Coronella), 71.

girondica (Coronella), 71 .

Glauconia, 6.

Glauconiidae, 6 .

Gonionotophis, 35 .

gracilis (Calamaria), 86 . 


\section{$-153$}

gracilis (C'a!lophis), 123. gracilis (Hydrophis), 112. graminea (Lachesis), 139. gramineus (Trimeresurus). 139. granulatus (Chersydrus), 16. granuliceps (Stenophis), 90. Grayia, 79. guentheri (Elapechis), 118. guentheri (Elapsoidea), 118. guentheri (Erythrolamprus), 109. gnentheri (Trachischium), 32. guianense (Rhinostoma), 98. guttatus (Coluber), 72.

haje (Naja), 120.

hainanensis (Simotes), 73.

halmahericus ('Tropillonotus), 28. halys (Ancistrodon), 137.

halys (Trigonoceplialus), 137.

Haplocercus, 33.

Haplopeltura, 127.

lardwickei (Enhydris), 114.

harriettae (Pseudelaps), 115.

leathi (Drymubins), 47.

heathi (Herpetorlyas), 47.

hebe (Lycodon), 37.

Helicops, 29.

Hemibungarus, 122.

Hemirhagerrhis, 99.

herminae (Ablabes), 78.

llerpetodryas, $5 \overline{\text {. }}$

herzi (Simotes). 50.

hessei (Elapechis), 118.

hessei (Elapsoidea), 118.

heterodermus (Chlorophis), 58.

heterodermus (Plilothamuus), 58.

Heterodon, $6 \dot{0}$.

heterolepilotus (Chlorophis), 58.

heterolepidotus (Philothammus), 58.

heterozonus (Elaps), 125.

heterurus (Stegonotus), 39.

hexagonotus (Xenelaphis), 47.

hierosolymitanus (Psammophis), 103.

hillebrandti (Atractaspis). 136.

himalayanus (Tropirlonotus), 26 .

Himantorles, 94.

hippocrepis (Zamenis), 46. lippus (CoInber), 22.

hispanica (Coronella), 71.

histricus (Lystrophis), 64 .

Homalocranium, 110.

Homalopsinae, 87.

Homalopsis, 88.

Homalosoma, 77.

Homorelaps, 125.

HoplocephaTus, 118.

hoplogaster (Chlorophis), 58.

Hormonotus, 37.

horridus (Crotalus), 142.

hortulanus (Corallus), 10.

hotamboeia (Leptodira), 94.

hugyi (Vipera), 132.

Hydrophinae, 112.

Hydrophis, 112.

Hydrops, 70.

Hydius, 112.

hypnale (Ancistrodon!, 137.

Hypsirhina, 87.

Hypsirhynchus, 60.

iberus (Tarboplis), 91.

ikaheka (Jicropechis), 118.

Ilysia, 14.

Ilysiidae, 14.

immaculatus (Oxyrrhopus), 96.

imperator (Boa), 12.

infernalis (Tropidonotus), 18.

infralineata (Geodipsas), 89.

infrataeniatus (Helicops), 30.

inornata (Cont a), 76.

inornatus (Ablabes), 76.

inornatus (Pseudoxenodon), 29.

insignitus (Coelopeltis), 101.

intermedia (Langaha), 90.

intermedius (Typhlops), 4.

intestinalis (Doliophis), 124.

iris (Calamaria), 84.

irregularis (Atractaspis), 136.

irregularis (Chlorophis), 58.

irregularis (Dipsarlomorphus), 93.

irregularis (Dipsas), 93.

irregularis (Philothamnus), ós.

Ischnognathus, 31 .

Itycyphus, 89. 
jaculus (Eryx), 13.

jaegeri (Rhadinaca). 67.

jamesoni (Dendraspis), 127.

japonicus (Dinodon), 38.

japonicus (Hemibungartus). 123.

jararaca (Trigonocephalus), 138.

jaranicus (Acrochordus): 16.

jerdoni (Distira), 114.

johni (Eryx), 13.

juliae (Liophis), 63.

karelini (Zamenis), 44.

kelleri (Hemirhagerrhis), 99.

kinkelini (Rhadinaea), 68.

kirtlandi (Dryophis), 107.

kirtlandi (Thelotornis), 107.

korros (Zamenis), 41.

knekenthali (Calamorhabdium), $8:$.

labnanensis (Simotes), 73.

lacertina (Coelopeltis), 101, 102.

Lachesis, 137.

lacrymans (Coluber), 103.

lacteus (Homorelaps), 125.

ladacensis (Zamenis), 44.

laetus (Dryophis), 106, 107.

laeviceps (Atheris), 135.

laevicollis (Dendrophis), วั.

laevis (Amblycephalns), 127.

laevis (Coronella), 71.

Lamprophis, 35.

lanceolata (Lachesis), 137.

Langaha, 90.

lansbergi (streptophorns), 31.

latastei (Vipera), 132.

laterale (Lycophidium), 36.

lateralis (Tropidonotus); 25.

laticaulatus (Platurus), 114.

latifasciatus (Dipsadomorphns), y3.

latirostris (Philodryas), 100.

lebetina (Vipera), 133.

Iehmanni (Atractus), 80.

lemniscatus Elapomorphus', 111.

lenzi (Typhlops), 2 .

leopardinus (Coluber), ว0.

leopardinus (Helicops), 30.

Leptocalamus, 75.
Leptorlira, 94.

Leptognathus, 128.

Leptophis, วy.

lencobalia (Fordonia), 89.

leucocephala (Calamaria), 84.

lencodira (Naja), 121.

lencomelas (Himantorles), 94.

Liasis, 7.

lichtensteini (Coluher), 48.

lineatus (Aporophis), 65.

lineatus (Boodon), 36.

lineatus (Cyclocorus), 35.

lineatus (Doliophis), 124.

Iineatus (Herpetodryas), 65.

lineatus (Typhlops), 1.

lineolatun (Taphrometopon), 103.

lineolatus (Dendrophis), 57.

limnei (Calamaria), $8 \overline{5}$.

liocercus (Leptophis), 60.

Lioheterodon, 29.

Liophis, 61.

longicauda (Ablabes), 79.

longiceps (Pseudorhabdium), 82.

longissinus (Coluber), 52.

loreatus (Hydrophis), 114.

lowi (Calamaria), 85.

lumbricalis (Typhlops), 3 .

lumbricoidea (Calamaria), $8: 3$.

Intea (Lachesis), 139.

luteus (Trimeresurus), 139.

Intrix (Homalosoma), 77.

luzonensis (Zaocys): 40.

Lycodon, 37.

Lyeognathopsis, 34 .

Lycophidium, 36.

Lystroplis, 64.

Lytorhynchus, 46 .

macclellandi (Callophis), 123.

macrolepis (Glanconia). 6.

macrolepis (Lachesis), 140.

macrolepis (Trimeresmus), 140.

macrophthalmus (Herpetodryas),

Maeropisthodon, 2-8.

Hacroprotodon, 106.

macrops (Pseudoxenodon), 29.

maculata (Denisonia), 117. 
maculata (Ungalia), 12.

maculatus (Cylindrophis), 14.

maculatus (Hydrus), 112.

maculatus (Oxyrhopus), 97.

maculatus (Streptophorus', 31.

maculatus (Tropidonotus), 27 .

maculiceps (Diemenia), 116.

madagascariensis (Boa), 12.

madagascariensis (Corallus), 11.

madagascariensis (Heterodon), 29.

madagascariensis (Lioheterodon', 29.

madagascariensis (Mimophis), 105.

madagascariensis (Typhlops), 2.

marlurensis (Platyplectrurus), 15.

mahfalensis (Mimophis), 105

major (Ablabes), 78.

maregravii (Elaps), 126.

margaritiferns (Drrmobius), 48.

martii (Homalopsis), 70, 80.

mauritanica (Vipera), 133.

mechowi (Xenocalamus), 111.

mellicii (Dasypeltis), 86.

melanocephala (Coronella), 110.

melanocephalum (Homalocranium), 110.

melanocephalum (Homalosoma), 75.

melanocephalus (Aspirlites), 9.

melanocephalus (Oligodon), 75 .

melanocephalus (Polyodontophis), 17.

melanoceploalus (Rbyncliocalamus), 75.

melanogaster (Pararhadinaea), 33.

melanoleuca (Naja), 120.

melanoleucus (Coluber), $\check{5} 4$.

melanostigma (Liophis), 63.

melanotus (Dipsadomorphus), 92.

melanotus (Liophis), 62.

melanozostus (Tropidonotus), 22.

melanurus (Coluber), 50), 53.

mentovarius (Zamenis), 42.

merremi (Coronella). 61, 66.

merremi (Rhatlinaea), 66.

merremi (Xenorion), 64.

meyerinki (simotes), 74 .

Jicrelaps, 111.

IIicropechis, 118.

microphulis (Coronella), 72.

mikani (Leptognatbus), 129.

miliaris (Crotalus), $1+1$. miliaris (Eryx, 13.

Jimophis, 105.

miniatus (Itycjphus), 89.

miniatus (Philodryas), 89.

miniatus (Tropidonotus), 27.

miolepis (Dromicus) 67.

mipartitus (Elaps), 127.

modlesta (Boa), 10.

morlesta (Calamaria), 83.

morlestum (Oxyrhabdium), 32.

morlestus (Ablabes), 76.

modestus (Cyclophis), 76.

modestus (Dendrelaphis), 58.

morlestus (Elapops), 112.

inorlestus (Helicops), 30.

modestus (Hormonotus). 37.

modestus (Lioheterolou, 29.

morlestus (Stegonotus) 38.

moclendorffi (Amblyceplaalns . 128.

moellendorffi (Coluber), כૅ.

muellenilorffi ('ynophis), 52.

moellendorffi (Pareas), 128.

moilensis (Coelopeitis), 102.

moilensis (Coluber), 102.

molurus (Python), 8

muniliger (Pammophis), 103.

monochrons (Calamaria), 83.

monozonus (Erytholamprus). 109.

monspessulana (Coelopeltis), 101.

monticola (Lachesis), 139.

mucosus (Ptyas), 41.

mucosus (Zamenis): 41 .

mucronatus (Typhlops), 4.

mucruso (Typhlops),

muelleri (Calamaria), 86.

muelleri (Diemenia), 11 .

muelleri (Micrelaps), 111.

muelleri (Psendelaps), 115.

muelleri (Stegonotus), 39.

mnelleri (Typlilops), 2.

multicinctus (Bungarus). 119.

multicinetus (Dipsarlomorphns), 93.

multimaculata (I)ipsas), y2.

multimaculata (Uipsina), 102

multimaculatum (Lycophidium), 37.

multimaculatus (Amplorhinus): 99.

multimaculatus (Dipsarlomorphus), 92. 
mu!timaculatus (Psammoplyylax), 99. multimaculatus (Rhamphiophis), 102. murinus (Eunectes), 12. muta (Lachesis), 137. mycterizans (Dryophis), 107.

Naja, 120.

najarlum (Zamenis), 44.

Nardoa, 7.

nasicornis (Bitis), 134.

nasus (Contia), 77.

nasuta (Langaha), 90.

nasutus (Dryinus), 106, 107.

natrix (Tropidonotus), 19.

nattereri (Thammorlynastes), 98.

nebulata (Dipsas), 79.

nebulata (Leptognathus), 79.

nebulata (Petalognathus), 79.

neuwiedi (Lachesis), 138.

neuwiedi (Oxyrrhopus). 97.

neuwieli (Xenoron), 63.

nigra (Silybura), 14.

nigricans (Stenostoma), 6.

nigricollis (Naja), 120

nigrocinctus (Elaps), 126

nigrolineatus (Typhl(,ps), t.

nigroluteus (Tretanorhinus), 30.

nigromarginatus (Zaocys), 40.

nototaenia (Amplorbinus', 99.

nummifer (Coluber), 45 .

nummifer (Zamenis!, 45.

nummifera (Atropos), 138.

nummifera (Lachesis), 138.

nympha (Clelia), 39.

nympha (Dryocalamns), 39.

obscura (Fleisclimannia), 69.

obsoletus (Coluber), 52.

obtusa (Vipera), 133.

obtusus (Coluber). 91.

obtusus (Tarbophis), 91.

occirlentalis (Leptophis), 59.

occipitalis (Cloelia), 97.

occipitalis (Furina), 124.

occipitalis (Oxyruhopus), 97.

occipitalis (Rladinaea), 67.

occipitomaculatus (Ischnognathus), 31 .

ocellatus (Tropidonotus), 24. octolineatus (Simotes), 73

Ogmodon, 115.

olfersi (Herpetodryas), 47.

olfersi (Philodryas), 100.

Oligodon, 74 .

olivacea (Coronella), 2 ?.

olivaceus (Boodon), 36.

oliraceus (Tropidonotus), 22.

Ophirlia, 1.

ordinatus (Tropidonotus), 18

ornata (Chrysopelea): 108.

ornata (Distira), 113.

ornatus (Hylrophis), 113.

ornatus (Hydrus), 112.

Oxybelis, 108.

oxycephalus (Coluber), 53.

Oxyrhabdium, 32.

Oxyrrhopus, 96.

oxyrrlynchus (Rhinophis), 15.

oxyrrhynchus (Uromacer), 60.

pallida (Naja), 120.

pallirlum (Homalocranium), 110.

palmarum (Dasypeltis), 86, 87.

pammeces (Typhlops', 2.

pantherinus (Ptyas), 47.

parallelus (Coluber): 46.

Pararhadinaea, 33.

parvifrons (Liophis), 63.

paucisquamatus (Typhlops!, 5.

pavimentata (Calamaria), 85.

paro (Echis), 13 s̆.

paronina (Leptognathus). 129.

pelamis (Hydrophis), 112.

perroteti (Xylophis), 32.

persa (Tropidonotus), 20.

personata (Leptodira), 95.

peruana (Leptognathus), 128.

peruviana (Tachymenis), 98.

Petalognathus, 79.

petolarius (Lycorlon), 96.

petolarins (Oxymhopus), 96.

phaenochalinus (Simotes), it.

philippinus (Ablabes), 78.

philippinus (Doliophis), 124

Philodryas, 99.

Philothamnus, 58. 
phocarum (Psendaspis), 40.

Phrynonax, 48.

picta (Lachesis), 138.

pictiventris (Helicops), 30.

picturatus (Tropidonotus), 19, 20.

pictus (Bothrops), 138.

pictus (Dendrophis), 56, 57.

pictus (Psammodynastes), 105.

piscator (Tropidonotus), 22.

piscivorus (Ancistrodon), 136.

planiceps (Homalocranium), 110.

planiceps (Rhinophis), 15.

Platurus, 114.

platurus (Hydrus), 112.

platyceps (Tropidonotus', 25.

Platyplectrurus, 15.

platyrrhinus (Heterodon), 65.

plumbea (Hypsirhina), 87.

plumbenm (Brachyrhytum), 97.

plumbens (Coluber), 97.

plumbiceps (Coronella), 71 .

plumbicolor (Jacropisthodon), 29.

poecilogyrus (Liophis), 61.

poecilonotus (Phrynonax), 49

poecilostoma (Tropidonotus). 52.

polygrammicus (Typhlops), 3.

Polyodontophis, 16.

ponticus (Tropidonotus), 20.

porphyracens (Coluber), 50.

porphyraceus (Pseudechis), 117.

porphyreus (Coluber), 117.

praeornatus (Dromophis), 102.

prasinus (Dryinus), 106.

prasinus (Dryuphis), 106.

prester (Vipera), 131.

proboscideum (Rhinostoma), 76.

pryeri (Tropidonotus), 26.

Psammodynastes. 105.

Psammophis, 103.

psammophis (Diemenia), 116.

psammophis (Psendelaps), 116.

Pseudablabes, 99.

Pseudaspis, 40.

Pseudeclis, 117.

Pseudelaps, 115.

Pseudorhabdium, 82.

Pseudoxenorton, 29. pullatus (Spilotes), 49.

pulneyensis (Silybura), 14.

pulverulentus (Psammodynastes), 105.

punctata (Coronella), 72.

punctatissima (Dipsas), 98.

punctatissimus (Psammodynastes), 98.

punctatolineata (Contia), 77.

punctatolineatus (Cyclophis), 77.

punctatus (Homorelaps), 125.

punctatus (Psammophis), 103.

punctatus (I'ython), 7.

punctatus (Typlilops), 4.

punctiventris (Tropidonotus), 19.

punctulatus (Dendrophis), 56, $\mathbf{5 7}$.

punctulatus (Psammophis), 103.

punicea (Lachesis), 140.

puniceus (Trigonocephalus), 140.

puniceus (Trimeresurus), 140.

purpurascens (Simotes), 73.

pustulatus (Thrasops), 599.

Python, 7.

Pythoninae, 7.

quadrilineatus (Boodon), 36 .

quadrilineatus (Coluber), 50).

quadrilineatus (Dromicodryas), 17.

quadrilineatus (Herpetodryas), 17.

quadrivirgatus (Colnber), 53.

quatuorlineatus (Coluber), 51.

quincunciatus (Tropilonotus), 22.

(quinquelineatus (Drymobius), 48.

radiatus (Culuber), 54 .

rappi (Ablabes), 78.

rappi (Drymobius), 48.

ravergieri (Zamenis), $4 \bar{\jmath}$.

reginae (Coronella), 63.

reginae (Lioplis), 63.

regius (Python), 9.

reinlarlti (Philodryas), 100.

renardi (Vipera), 130.

reticulata (Diemenia), 117.

reticulatus (Atractus), 80.

reticulatus (Python), 8.

reticulatus (Stegonotus), 38.

reticulatus (Typhlops), 3.

reutei (Typhlops), 2. 
thabrlocephalus (Ophis). 63.

Rhabdophidium, 81.

Rhachioclontinae, 86 .

Rhadinaea, $6 \tilde{0}$.

Rhamphiophis, 102 .

Rhinophis, 15.

Rhinostoma. 98.

rhinostoma (Simophis), 76 .

rhodogaster (Enicognatbus), 16.

rhorlomelas (Macropisthorlun), 28.

rhodoplemron (Chrysopelea), 108.

rhorlorhachis (Zamenis), 44.

rhodostoma (Ancistrodon). 137.

rhorlostoma (Trigonocephalus). 137.

rhombeata (Coronella), 101.

rhombeata (Lachesis), 137.

rhombeatus (Aspidelaps), 130.

rhombeatus (Causus): 130.

rhombeatus (Psammophylax), 101.

rhombeatus (Trimerorhinus). 101.

rhombifer (Irymolius), 48.

rhombifer (Uxyrrhopus), 96.

rhomboidea (Calamaria), 85.

rhynchops (Cerberus), 88.

rostrata (Atractaspis), 136.

rothi (Ablabes), 77.

rothi (Contia), 77.

ruber (Typhlops), 3.

rueppelli ('lyphlopss), 3.

rufescens (C'oronella). 3à.

rufescens (Crotaphopeltis). 94.

rufescens (Leptodira), 94.

rufiventris (Dromicus), 61.

rufodorsatus (Cohuber), ธ0.

rufozonatus (Dinorlon), 38.

rufulus (Ablabophis), 35.

rufus (Cylintrophis): 14.

ruschenbergi (Corallus), 10.

russelli (Vipera), 133.

rusticus (Oxyrrhopns), 97.

salomonis (Dendrophis), 56. sagittifera (Rlarlinaea), 66. samarensis (Naja), 122.

saurita (Herpetodryas), 19.

saurita (Tropidonotus), 19.

sauromates (Coluber), 51. savignyi (Tarbophis), 91.

sayi (Coronella), 71 .

sayi (Herpetulryas), 72.

scabra (Lasypeltis), 86.

scalaris (Coluber), 54 .

scalaris (Tropidonotus), 18.

schinzi (Typhlops), $\bar{~}$

schistosum (Homalocranium), 110)

schistosus (Helicops), 29.

schistosns (Hydrophis), 114.

schlegeli (Lachesis), 141.

schmackeri (Coluber), 53.

schueideri (Yython), 8.

schneileri (Vipera), 134.

schokari (Psammophis), 103.

schotti (l'hilodryas), 100.

schrencki (Coluber), 51.

scutatus (Tropidonotus), 21.

scutifrons (Glauconia), 6.

scytale Calamaria) 33.

scytale (Ilysia), 14.

sebae Python), 8 .

sebae (Streptophorus), 32.

sechellensis Lycognathopsis), 34.

semiannulata ('alamaria), 84.

semiannulata Leptodira), 91.

semianmulatus (Tarbophis), 91.

semicarinatus (Ablabes), 78.

semicinctum (Homaloeranium), 110.

semicinctus (Tropidonotus), 19.

semiloliatus (Geophis), 81.

semifasciatus (Bungarus), 119.

semimaculata (Contia), 77.

semimaculatus (Ablabes), 77.

semioruata (Coronella), 71.

semivariegatus (Philothannus), 58.

seoanei (Vipera), 131.

severus (Ophis), 64.

severus (Xenodon), 64.

sexcarinatus (Herpetodryas), 55.

sibilans (Psammophis), 103, 104

siculus (Tropidonotus), 20.

signata (Denisonia), 117.

signatus (Simotes), 74.

Silybura, 15.

simoni (Onychocephahus),

simoni (Typhlops), ó 
Simophis, 76.

Simotes, 73.

simus (Heterodon), 65.

sirtalis (Tropidonotus), 18.

Sistrurus, $1+1$.

smaragdina (Gastropyxis), 59.

smaragdina (Hapsidophrys), 59.

smythi (Gravia), 79.

spilogaster ('Tropidonotus), 27.

Spilotes, 49.

spilutes (Python), 7.

spinalis (Achalinus), 33.

spixi (Elaps), 126.

sputatrix (Naja), 121.

squamiger (Atheris), 135.

Stegonotus, 38.

Stenophis, 90.

Stenorhina, 111.

stolatus (Tropidonotus), 26.

storerioides (Ischnognathus), 31.

Streptophorus, 31.

striatus (Epicrates), 10.

striatus (Lycodon), 37.

strigata (Lachesis), 139.

stumpffi (Dromicus), 2อ.

stumpffi (Tropidonotus), 25.

Stypurhynchus, 28.

subcinctus (Lycolon), 38.

sulggriseus (Oligodon). 75.

sublineatus (Oligodon), 7ó.

subminiatus (Tropidonotus), 26.

subniger (Zamenis), 44.

subpunctatus (Polyorlontophis), 17.

sulphureus (Phryonax), 48.

sumatrana (Calamaria), 83.

sundevalli (Glauconia), 6.

surinamensis (Elaps), 125.

syriacus (Tarboplis), 91.

Tachymenis, 98.

taeniatus (Zamenis), 42.

taeniogastra (Rhadinaea), 66.

taeniolata (Coronella), 67.

taeniurus (Coluber), 51.

taeniurus (Liophis), 61.

taeniurus (Oligodon), 75 .

'Taphrometopon, $1 \cup 3$
Tarboplis, 91.

templetoni (Oligodon), 75.

temuis ('T'yphlops), 2.

terlineata (Calamaria), 81

terrificus (Crotalus), 142.

terrificus (Donlrelaphis), 57.

tessellata (Calamaria), 85.

tessellatus (Tropidonotus), 23.

Tetralepis, 35.

tetrataenia (Doliophis), 123.

totrazomus (Erytlrolamprus), 109,

textilis (Djemenia) 117.

Thamnodynastes, 98 .

thebaicus (Eryx), 13.

Thelotornis, 107.

thomensis (hilothamnus), 53.

'Thrasop's, 59.

timrinus (Tropidonotns), 25.

Tomodon, 99.

torifuatus (Airactus), 80.

torinatus (Leptocalamus), 75.

torquatus (Polyodontophis), 16.

trabalis (Zamenis), 43.

Truchischium, 32.

trachyprocta (Aspillura), 33.

trimsversalis (Calamaria), 85.

tris vancoricus (Lycodon), 38.

Tretanorlhinus, 30.

trevelyanus (lihinophis), 15.

triangularis (Grayia), 79.

triangularis (Hydrops), 70.

triangnligerus (Tropidonotus), 21.

triangulum (Coronella), 72.

tricolor (Ablabes), 78.

trigemimus (Oxyrrhopus), 96i.

trigonatus (Dipsulomorphus), y2.

trigonocephala (Lachesis), $1 \neq 0$.

trigonocepha! us (Trimeresurus), 140 .

trilineatus (Atractus), 81.

trilineatus (Dromicodryas), 17.

trilineatus (Herpetodryas), 17

trilineatus (Trimerorhinus), 101.

Trimerorhinus, 101.

trinotatus (Simotes), 73.

tripudians (Naja), 121.

tristis (Dendrelaphis), 57.

Tropidonotus, 18. 


\section{$-160$}

troscheli (Typllops), 3.

truncatus (Styporhynchus), 28.

truncatus (Tropidonotus), 28.

tschudii (Elaps), 125.

Typhlopidae, 1.

Typhlops, 1.

typhlus (Liophis), 62.

typus (Dispholidus), 108.

undulata (Rhallinaea), 67.

Ungalia, 12.

unicolor (Boodon), 36.

unicolor (Fordonia), 89.

unicolor (Xenopeltis), 16.

univirgatus (Callophis), 123

Uromacer, 60.

Uropeltidae, 15.

Urotheca, 68.

ursinii (Vipera), 130.

valakadien (Enhydrina), 114.

varia (Eclis), 135.

variabilis (Coluber), 49.

variegatus (Boodon). 36.

variegatus (Python), 7.

variegatus (Tropidonotus), 19.

varius (Typhlops), 5.

ventrimaculata (Leptognathus), 129.

ventrimaculatus (Zamenis), 44.

venustissimus (Erythrolamprus), 109.

vermicularis (Typhlops), 2.

vermiformis (Calamaria), 83.

vernalis (Contia), 76.

versicolor (Calamaria), 85.

versicolor (Zamenis), 46.

vibakari (Tropidomotus), 21 .

Vipera, 130.

vipera (Cerastes), 135.

Viperidae, 130.

viperina (Distira), 114.

Viperinae, 130.

viperinus (Hydrophis), 114.

viperinus (Tropidonotns), 24.

virgulata (Calamaria), 83.

vuriciitlasus (Coluber), 43.

viridiflarus (Zamenis), 43.

viridis (Chondropython), 9.

vilidis (Dendraspis), 127.

viridis (Trigonocephalus), 139.

viridissimus (Herpetodryas), 100.

viridissimus (Philodryas), 100.

vitianns (Ogmodon), 115 .

vittata (Rhadinaea), 67.

vittatus (Tropidonotus), 22, 26.

vivax (Aelurophis), 91.

vivax (Tarbophis), 91.

rossi (Gonionotophis', 35.

vossi (Gonionotus), 35.

wagleri (Lachesis), 141.

weigeli (Dipsas), 129.

werncri (Aparallactus), 111.

wiedi (Typhlops), 4.

xanthina (Vipera), 133.

Xenelaphis, 47.

Xenocalamns, 111.

Xenodon, 63.

Xenopeltidice, 16.

Xenopeltis, 16.

Nylophis, 32.

Zamenis, 41 .

Zaocys, 40.

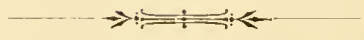

Druckfehler.

Anf pag. 59 ist unter der Zeile:

Genus XLVIII. Gastropyxis Cope.

aus Versehen ansgefallen

$$
\text { ,1. Gastropyxis smaragdina (Schlg.)“, }
$$
was wir zu verbessern bitten. 


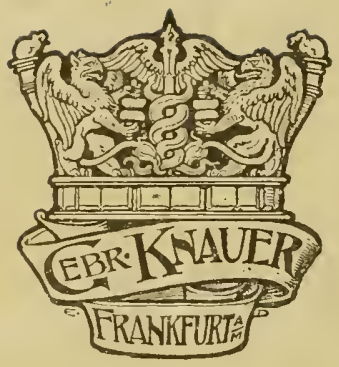







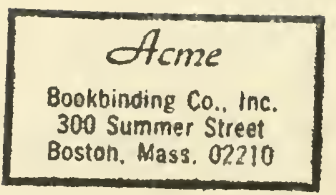




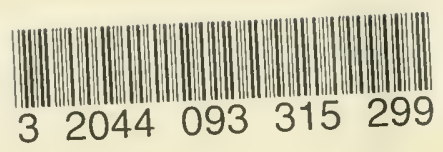


